\title{
Jahrbuch der berufs- und wirtschaftspädagogischen Forschung 2020
}

Eveline Wittmann, Dietmar Frommberger, Ulrike Weyland (Hrsg.)

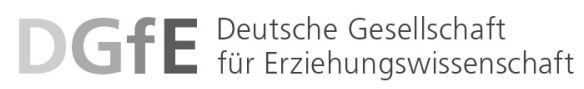


Jahrbuch der berufs- und

wirtschaftspädagogischen Forschung 2020 
Schriftenreihe der Sektion

Berufs- und Wirtschaftspädagogik

der Deutschen Gesellschaft für

Erziehungswissenschaft (DGfE) 
Eveline Wittmann

Dietmar Frommberger

Ulrike Weyland (Hrsg.)

Jahrbuch der berufs- und

wirtschaftspädagogischen

Forschung 2020

Verlag Barbara Budrich

Opladen • Berlin • Toronto 2020 
Bibliografische Information der Deutschen Nationalbibliothek

Die Deutsche Nationalbibliothek verzeichnet diese Publikation in der Deutschen

Nationalbibliografie; detaillierte bibliografische Daten sind im Internet über

http://dnb.d-nb.de abrufbar.

\section{Veröffentlicht mit Unterstützung der Käthe und Ulrich Pleiß-Stiftung}

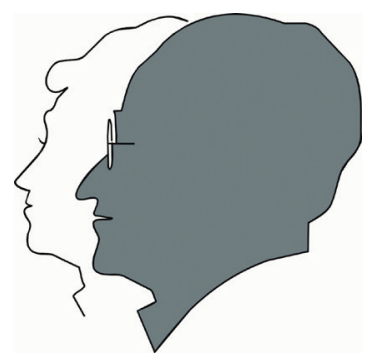

(C) 2020 Dieses Werk ist bei der Verlag Barbara Budrich GmbH erschienen und steht unter der Creative Commons Lizenz Attribution-ShareAlike 4.0 International (CC BY-SA 4.0): https://creativecommons.org/licenses/by-sa/4.0/.

Diese Lizenz erlaubt die Verbreitung, Speicherung, Vervielfältigung und Bearbeitung bei Verwendung der gleichen CC-BY-SA 4.0-Lizenz und unter Angabe der UrheberInnen, Rechte, Änderungen und verwendeten Lizenz.

www.budrich.de

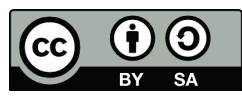

Dieses Buch steht im Open-Access-Bereich der Verlagsseite zum kostenlosen Download bereit (https://doi.org/10.3224/84742437).

Eine kostenpflichtige Druckversion (Print on Demand) kann über den Verlag bezogen werden. Die Seitenzahlen in der Druck- und Onlineversion sind identisch.

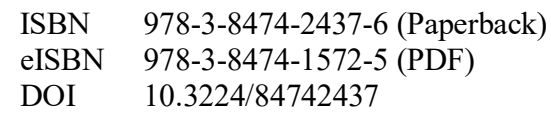

Umschlaggestaltung: Bettina Lehfeldt, Kleinmachnow - www.lehfeldtgraphic.de Druck: docupoint GmbH, Barleben

Printed in Europe 


\section{Inhaltsverzeichnis}

Vorwort: Forschungserträge und Forschungsperspektiven der Berufs- und Wirtschaftspädagogik

\section{Teil I: Reflexion der Disziplin: Außenperspektive}

Andrea Sailer

Literarische Zusammenschau zu den Tagungsbeiträgen

\section{Teil II: Erträge aus den Forschungszusammenhängen der Berufs- und Wirtschaftspädagogik: Finanzkompetenz und Bildung für nachhaltige Entwicklung}

Bärbel Fürstenau, Mandy Hommel, Manuel Förster, Andreas Kraitzek, Eveline Wuttke, Carmela Aprea, Michelle Rudeloff und Christin Siegfried Messung von Finanzkompetenz - Ergebnisse eines Symposiums

Andreas Fischer, Marc Casper, Karina Kiepe, Harald Hantke, Jan Pranger und Sören Schütt-Sayed

Theoretische Reflexionen zur Didaktik der Berufsbildung für nachhaltige Entwicklung (BBNE) aus der Perspektive der Modellversuchsforschung

\section{Teil III: (Zwischen)Ergebnisse aus Projekten}

\section{Berufsverbleib und Berufsorientierung}

Dana Bergmann

Verarbeitung von Studienabbrüchen innerhalb beruflicher Entwicklungsprozesse - eine berufsbiografische Analyse

Silke Lange

Vorzeitige Vertragslösungen aus der Perspektive der Auszubildenden ein Vorschlag für einen subjektorientierten Erklärungsansatz

Leonie Bogaczyk, Marie Schröder, Thomas Retzmann und Thomas Bienengräber

Theoretische Grundlagen und empirische Befunde zum Wissensmanagement in der inklusiven Berufsorientierung 
Heike Jahncke, Florian Berding, Jane Porath und Christian Steib Berufsdarstellungen in Vorabendserien zwischen 1990 und 2018 als Informationsquellen über Berufe

\section{Berufliche Didaktik und Curriculum}

Susanne Korth, Svenja Noichl und Volker Rexing

Inklusive Lernumgebungen mit digitalen Medien in Überbetrieblichen

Berufsbildungsstätten der Bauindustrie

Jutta Mohr, Isabelle Riedlinger und Karin Reiber

Die Bedeutung der Digitalisierung in der Neuausrichtung der pflegerischen Ausbildung - Herausforderungen für die berufliche Pflege im Kontext der Fachkräftesicherung

\section{Professionalisierung von Lehrkräften beruflicher Schulen}

Andrea Burda-Zoyke und Immo Degner

Erwartungen an Praxissemester im Studium für ein Lehramt an

beruflichen Schulen - eine qualitative Studie im Rahmen der

Studiengangentwicklung

Josephine Berger und Birgit Ziegler

Studienzufriedenheit und Studienerfolg im ersten Studienjahr -

Studierende im Lehramt an beruflichen Schulen und an Gymnasien

im Vergleich

\section{Teil IV: Reflexion der Disziplin: Innenperspektive}

Silke Lange, Dietmar Frommberger, Ulrike Weyland und

Eveline Wittmann

Die Qualitätsoffensive Lehrerbildung aus der Perspektive der

beruflichen Lehrerbildung

Herausgeberschaft

Autorinnen und Autoren 


\section{Forschungserträge und Forschungsperspektiven der Berufs- und Wirtschaftspädagogik}

Seit dem Jahr 2012 gibt die Sektion für Berufs- und Wirtschaftspädagogik der Deutschen Gesellschaft für Erziehungswissenschaft (DGfE) ein ,Jahrbuch der berufs- und wirtschaftspädagogischen Forschung" heraus. Die Aufgabe des Jahrbuchs besteht darin, die im Rahmen der Sektionstagungen geführten Diskussionen zu dokumentieren und damit einen Einblick in den aktuellen Stand der Berufsbildungsforschung in ihrer Breite und Tiefe zu geben. Die im September 2019 an der Karl-Franzens-Universität Graz durchgeführte Jahrestagung der Sektion Berufs- und Wirtschaftspädagogik der DGfE erfreute sich einer äußerst guten Resonanz. Das aktuelle Jahrbuch präsentiert aus der Bandbreite der Vorträge und Symposien ausgewählte Beiträge, die wie gewohnt einem doppelten Review-Verfahren unterzogen wurden.

Das Jahrbuch weist in diesem Jahr einige Besonderheiten auf. Eingeleitet wird es durch die literarische Zusammenschau der Schriftstellerin Andrea Sailer, die mit ihren nachdenklich-persönlichen Anmerkungen das Publikum der Grazer Tagung begeisterte und gekonnt, mit Witz und betroffen machend blinde Flecken gegenwärtiger Diskurse der BWP auslotet. Wir freuen uns sehr, dass Frau Sailer der Sektion ihr Manuskript zur Verfügung gestellt hat und drucken diesen Beitrag auf ausdrücklichen Wunsch aus dem Mitgliederkreis als Faksimile ab.

Erstmalig hat der Vorstand auf der Mitgliederversammlung dazu aufgerufen, Beiträge über gesamte Symposien einzureichen, um auf diesem Weg mit dem Jahrbuch verstärkt die wissenschaftliche Diskussion in der Disziplin voranzubringen. Drei Autorenkollektive sind der Anregung gefolgt. Zwei dieser Beiträge zu den Themen „Finanzkompetenz/Financial Literacy“ und „Bildung für nachhaltige Entwicklung" präsentieren wir zu Beginn des Bandes, da diese im Sinne einer retrospektiven und systematisierenden wissenschaftlichen $\mathrm{Zu}$ sammenschau zentrale wissenschaftliche Forschungsbereiche der Disziplin in den vergangenen Jahren aufgreifen.

In diesem Sinne adressieren zunächst Bärbel Fürstenau, Mandy Hommel, Manuel Förster, Andreas Kraitzek, Eveline Wuttke, Carmela Aprea, Michelle Rudeloff und Christin Siegfried Möglichkeiten, Finanzkompetenz zu messen, d. h. die Fähigkeit, mit finanziellen Belangen angemessen umzugehen, wobei sie diese Kompetenz als für das langfristige Wohlergehen von Individuen und Volkswirtschaften wesentlich ansehen. Zunächst wird verdeutlicht, welche nationalen und internationalen Messverfahren für Finanzkompetenz zur Verfügung stehen. Ein Forschungsdefizit bestand demzufolge bislang, neben situativen Messverfahren, in solchen Verfahren, die nicht-kognitive Komponenten sowie prozessbezogene Aspekte von Finanzkompetenz messen. Im Beitrag 
werden situations- bzw. entscheidungsbasierte Ansätze zur Messung von Finanzkompetenz sowie prozessbasierte Messansätze genauer ausgeführt. Insgesamt liefert der Artikel umfassende Hinweise dazu, wie die Forschung zur Messung von Finanzkompetenz weiterentwickelt werden könnte, insbesondere auch mit Blick auf den festzustellenden Gender Gap, der sich in verschiedenen Instrumenten unterschiedlich abbildet, auf informelles Lernen in digitalen Umgebungen sowie auf Fehlkonzepte.

Ebenfalls als Zusammenschau eines Symposiums behandeln Andreas Fischer, Marc Casper, Karina Kiepe, Harald Hantke, Jan Pranger und Sören Schütt-Sayed aus einer Perspektive der Modellversuchsforschung die Didaktik der Berufsbildung für nachhaltige Entwicklung. Nachhaltige Entwicklung verstehen sie als aushandlungsbedürftige regulative Idee. Sie verfolgen, ausgehend von der Förderlinie BBNE des Bundesinstituts für Berufsbildung (BIBB), im Sinne einer theoretischen Gesamtreflexion für den Bereich kaufmännischen Handelns die Frage, wie nachhaltige Entwicklung als Zieldimension in der Breite der Berufsbildung etabliert werden kann. Dazu thematisieren die Autor*innen zunächst die fachwissenschaftliche Fundierung einer Berufsbildung, wobei sie beispielhaft anhand des Modellversuchs „Geschäftsmodell- und Kompetenzentwicklung für nachhaltiges Wirtschaften im Handel (GeKoNaWi)" den Implikationszusammenhang fachdidaktischer Bezüge verdeutlichen. Anschließend behandeln sie anhand der systemischen Visualisierung aus dem Modellversuch Pro-DEENLA die Frage, inwieweit methodisch Perspektivwechsel, Widerspruchsbewusstsein und transformatorische Aushandlungsprozesse ermöglicht werden können. Das vierte Kapitel führt die Ansätze zu der Überlegung zusammen, für eine Bildung für nachhaltige Entwicklung sei es grundlegend, die auf Struktur und Rationalität abhebende Kompetenzorientierung mit der Entwicklung humanistischer Werte und Anliegen im Sinne eines Emotionalität, Metaphern und Improvisation betonenden Lernens in ein positives Spannungsfeld zu bringen.

Unter den Einzelbeiträgen dominieren in diesem Jahrbuch Beiträge, die sich mit den Thematiken Berufsverbleib und Berufsorientierung beschäftigen. Zwei Artikel haben subjekttheoretische Perspektiven auf Abbrüche zum Gegenstand. Mit den berufsbiographischen Auswirkungen von Studienabbrüchen und Neuanfängen im Bereich der Berufsausbildung befasst sich der Beitrag von Dana Bergmann. Sie thematisiert mittels autobiographisch-narrativer Interviews mit ehemaligen Studierenden identitätsbezogene Deutungsmuster von Studienabbrüchen in individuellen Berufsbiografien. Dabei konzipiert sie unter der Kategorie ,,personale Mobilität“ Bilanzierungen von Studienabbrüchen als einen identitätsentwickelnden (Bildungs-)Prozess, in welchem sich das Subjekt auf eine berufliche Platzsuche begibt.

Sodann behandelt Silke Lange vorzeitige Ausbildungsvertragslösungen aus subjektorientierter Perspektive, indem sie auf die sinn- und identitätsstif- 
tende Wirkung von Ausbildung abhebt. Ausgehend von Befunden einer empirischen Untersuchung zur Eingangsphase der Berufsausbildung intendiert der Beitrag, einen Strukturierungsvorschlag für personenbezogene Bedingungsfaktoren vorzeitiger Vertragslösungen zu liefern. In der empirischen Studie wurden problemzentrierte Interviews mit 18 Auszubildenden zu Ausbildungsbeginn im Kfz-Mechatroniker-Handwerk geführt. Im Anschluss an die Studie wird postuliert, dass die Gründe für Vertragslösungen in der Überforderung durch unzureichende individuelle Bedürfnisbefriedigung liegen sowie im zunehmenden Unvermögen, die Bedürfnisbefriedigung wiederherzustellen.

Aus einem Symposium der Grazer Tagung ist der Beitrag von Leonie Bogaczyk, Marie Schröder, Thomas Retzmann und Thomas Bienengräber entstanden, indem es um die Fragestellung geht, wie durch Wissensmanagement die Berufsorientierung von Schüler*innen mit Förderbedarf unterstützt werden kann. Die Autor*innen konzipieren Wissensmanagement mit Probst et al. über die Elemente Wissensziele, Wissensidentifikation und Wissenserwerb. Sie präsentieren qualitative empirische Befunde aus 18 Gruppendiskussionen mit Teilnehmenden unterschiedlicher einschlägiger Professionen und Institutionen, die inhaltsanalytisch ausgewertet wurden. Forschungsdesiderate werden u. a. im Bereich von Lehrerfortbildung und Schulentwicklung gesehen.

Heike Jahncke, Florian Berding, Jane Porath und Christian Steib widmen sich anderen Informationsquellen der Berufsorientierung, nämlich Berufsdarstellungen, wie sie in bekannten Vorabendserien erfolgen. Ausgehend von den durch die Autor*innenen untermauerten Annahmen, dass Fernsehpräsentation das Image eines Berufes beeinflussen kann, der Fernsehkonsum der jüngeren Generation weiterhin einen bedeutsamen Umfang aufweist und Fernsehkonsum v. a. bei fehlenden praktischen Erfahrungen eine wesentliche Quelle berufswahlrelevanter Informationen darstellen kann, zeichnet der Beitrag nach, wie sich in den vergangenen 20 Jahren Informationen über Berufe in Fernsehsendungen entwickelt haben. Er fragt nach dem Spektrum an Berufen, Tätigkeiten innerhalb der dargestellten Berufe sowie der Charakterisierung der Berufe in populären Vorabendserien. Diese Fragen begründen die Autor*innen systematisch entlang der bestehenden empirischen Befundlage. Die Ergebnisse zeigen, dass die ausgewählten Vorabendserien eine große Zahl an Berufen darstellen, vorrangig aus dem kaufmännisch-verwaltenden Berufsfeld. Jedoch stellen sie lediglich einen geringen Anteil des Tätigkeitsspektrums der Berufe und damit keine belastbare Wissensbasis für die Berufswahl dar, obgleich die Charakterisierungen im Wesentlichen denen des Holland-Modells entsprechen und außerdem geschlechtsspezifische Stereotypen vermeiden.

In zwei Beiträgen des diesjährigen Jahrbuchs dominieren didaktische und curriculare Perspektiven. Susanne Korth, Svenja Noichl und Volker Rexing befassen sich mit dem Einsatz digitaler Medien in inklusiven Lernumgebungen in Überbetrieblichen Berufsbildungsstätten der Bauindustrie, auch vor dem 
Hintergrund des Rekrutierungsbedarfs dieser Branche. Im Rahmen eines Design-Based-Research-Ansatzes zielen die Autor*innen auf digitale Lernsettings $a b$, die neuartige Formen für ein gemeinsames Lernen von Auszubildenden mit und ohne Lernbehinderung bereitstellen. Um kognitive Lernbarrieren zu ermitteln, führen die Autor*innen inhaltliche Anforderungsanalysen und solche zu den Lernervoraussetzungen durch, konstruieren darauf basierend digitale Lernumgebungen und stellen die Ergebnisse in Form einer Lern-App zu unterschiedlichen Fachinhalten des Berufsbereichs zur Verfügung, welche auf Basis von Evaluationsdaten weiterentwickelt wird. Aktuell findet eine Pilotierung in der Berufsgrundbildung mit etwa 100 Auszubildenden statt.

Jutta Mohr, Isabelle Riedlinger und Karin Reiber befassen sich im Kontext der Fachkräftesicherung mit Erwartungshaltungen zur Digitalisierung im Bereich der Pflegeberufe, dem Stand der digitalen Transformation der Berufspraxis und der diesbezüglichen Ausgestaltung der generalistischen Pflegeausbildung. Dazu analysieren sie die Ergebnisse einer Delphi-Studie sowie zweier Betriebsfallstudien, welche auf Basis von Interviews organisational relevanter Gruppen und mittels Gruppendiskussionen von Pflegekräften durchgeführt wurden. Digitalisierung wird demzufolge vorrangig bezogen auf Prozesse, Dokumentation und Planung und kaum im Hinblick auf die Entlastung von Pflegekräfte oder die Berufs- und Arbeitgeberattraktivität. Die curricularen Analysen ergeben, dass die jüngst verabschiedeten Rahmenpläne der Pflegeausbildung zwar Anwendungsbezüge konkretisieren, jedoch kaum solche Kompetenzen, die für eine entlastende und zugleich kritisch-konstruktive Anwendung digitaler Technologien in der Pflegepraxis erforderlich wären.

Zwei Beiträge thematisieren die professionelle Entwicklung von Lehrkräften beruflicher Schulen. Andrea Burda-Zoyke und Immo Degner untersuchen im Rahmen einer qualitativen Interview-Studie mit 16 Proband*innen Erwartungen, die unterschiedliche Akteure zu Beginn eines Studiengangentwicklungsprozesses an Praxissemester im Studium für ein Lehramt an beruflichen Schulen stellen. Die Studie verdeutlicht die Heterogenität der Zielsetzungen unterschiedlicher universitärer Stakeholder und unterstreicht Vorbefunde anderer Studien, wonach Studierende insbesondere Überforderungs- bzw. Überlastungsängste aufweisen, wohingegen die Professor*innen eine Herausforderung in der zielgerichteten Betreuung und theoriegeleiteten Reflexion des praktischen Handelns sehen.

Josephine Berger und Birgit Ziegler beleuchten Selbstselektionsprozesse beim Zugang zum Lehramtsstudium in Abhängigkeit von motivationalen Voraussetzungen und von Vorstellungen zum professionellen Kern des Lehrendenberufs und analysieren vergleichend die Studienzufriedenheit und den Studienerfolg im ersten Studienjahr bei Studierenden im Lehramt an beruflichen Schulen und an Gymnasien. Sie untersuchen die Studienzufriedenheit und abbruchsquoten im Zusammenhang mit der Implementation neuer Beratungs- 
elemente - Peer-Beratung, Online-Self-Assessment, individuelle berufsbezogene Eignungsberatung - sowie motivationale Faktoren in einem Panel von 102 Studierenden des beruflichen und des gymnasialen Lehramts. Den Ergebnissen zufolge nutzen Studierende des beruflichen Lehramts weniger Informationsquellen, scheinen zu Studienbeginn andere Erwartungen als Studierende des gymnasialen Lehramts aufzuweisen und sind nach dem ersten Studienjahr signifikant unzufriedener als Studierende des gymnasialen Lehramts, worauf die Inhalte der beruflichen Fachrichtung und der Workload einen hohen Einfluss haben. Die Verfasserinnen schlagen vor, verstärkt Brückenkonzepte für den Übergang ins berufliche Lehramt zu entwickeln.

Wir schließen den Band mit einem Beitrag, der, ebenfalls die Professionalisierungsthematik tangierend, der Selbstreflexion der Disziplin dient - auch dies ein Novum. „In eigener Sache“ des Sektionsvorstands wird im Beitrag von Silke Lange, Dietmar Frommberger, Ulrike Weyland und Eveline Wittmann die Diskussion der Mitglieder um die „Qualitätsoffensive Lehrerbildung" aufgegriffen. Auf der Grundlage von veröffentlichten Daten zu den Projekten der Qualitätsoffensive Lehrerbildung und auf der Basis von Befragungen der Mitglieder der Sektion wird die Frage nach der Sichtbarkeit und Relevanz der beruflichen Lehrerbildung in der Qualitätsoffensive Lehrerbildung diskutiert. Gemessen wird der Stellenwert anhand des Anteils positiv beschiedener Projekte sowie der Anzahl und des Umfangs der geförderten Projekte zur beruflichen Lehrerbildung. Die Analysen zeigen, dass es mit der dritten Förderrichtlinie und der darin enthaltenen Schwerpunktsetzung gelungen ist, die berufliche Lehrerbildung stärker zu berücksichtigen, als in den ersten beiden Förderrunden. Dennoch bleibt das Fördervolumen der beruflichen Lehrerbildung hinter der allgemeinen Lehrerbildung und der Digitalisierung als zweitem Schwerpunkt der dritten Förderrunde zur Qualitätsoffensive Lehrerbildung zurück. Trotzdem resümieren die Autor*innen, dass ,die Sichtbarkeit und Wertschätzung der beruflichen Lehrerbildung im Programm der Qualitätsoffensive Lehrerbildung gestiegen sind."

Der Vorstand der Sektion Berufs- und Wirtschaftspädagogik der DGfE dankt Prof. Dr. Michaela Stock (Karl-Franzens-Universität Graz) sowie den dortigen Mitarbeiter*innen sehr herzlich für die Ausrichtung der Sektionstagung. Unser besonderer Dank geht auch an die Käthe und Ulrich Pleiß-Stiftung, die das Jahrbuch in diesem Jahr erstmalig großzügig finanziell unterstützt. Wir danken den Gutachter*innen, die die eingegangenen Beiträge in gewohnt akribischer Manier unter die Lupe nahmen und viele konstruktive Hinweise zur Verbesserung der Texte lieferten. Schließlich danken wir Anya Prommetta sehr für die engagierte redaktionelle Bearbeitung der Beiträge.

München, Osnabrück und Münster, im Juli 2020

Eveline Wittmann, Dietmar Frommberger und Ulrike Weyland 

Jahrestagung Sektion Berufs- und Nirtschaftspäda= gogik Graz 2019 - Institut für N irtschaftspädagogik (25.-27. September 2019, UNI Graz)

\author{
Andrea Sailer: \\ "Literarische-Zusammenschau zu \\ den Tagungsbeiträgen"
}

$\therefore:$ Vortrag

27. September 2019 
I. Vorsicht: Persönlich!

Manchmal sagt man freudig etwas zu, um bald darauf fest= zustellen, dass einem alles damit Zusammenhängende Angst macht. Angst hat viel mit-Unsicherheit und Unwissen zu tun. Angst ist ein Gefühl, das ich im Mathematikunter= richt kennengelernt habe. In einem Tagungsprogramm für Berufs - und Wirtschaftspädagogik hat Angst eigentlich keinen Platz. Im Berufsalltag wohl generell nicht. Und im Iueben?

Zum Glǐcck trägt mein Vortrag den -bereits vorgegebenen -Titel: "Literarische Zusammenschau zu den Tagungsbei= trägen.". Was für ein gnädiger Witel! Vielen Dank, Frau Prof. Dr. Stock! Allein das Wort "literarisch" ist-ja, wie Kunst überhaupt, ein klassischer Joker. Und " $\mathrm{Zu}=$ sammenschau" wirkt ungleich freundlicher als "Zusammen= fassung". Was hätte auch eine wie ich - gelernte Philo= sophin und freie Schriftstellerin - aus der beklemmenden Fứlle von Beiträgen zusammenfassen können? Ich, die ich bei den meisten Vortragstiteln bloß zusammenfahre, weil ich-nicht fassen kann, womit sich Menschen ein Ieben lang freiwillig beschäftigen, und in welchen schwierigen Bereichen sie sich auskennen. -.-.-Ich habe das Wort "Zusammenschau" sehr ernst genommen, mir das gesamte Programm immer wieder ganz genau ange= schaut und versucht, die hoffentlich einfacheren $\mathrm{Be}=$ deutungen hinter den komplisierten Piteln zu entschlius= seln; zu verstehen, worum es dabei geht. Philosophen machen es normalerweise ja umgekehrt: Sie drücken ein= fachste Sachverhalte so kompliziert aus, dass sie kein Mensch mehr versteht. Warum sie das tun? -Ganz einfach: Weil sie es können. 
Um Können geht es hier auch, in allen Facetten. Ums Iehren und Iernen, und um das, was lebenslang und lebenswichtig daran hängt: Arbeit, teistung, Geld.

Es ist mutig, fast kühn, eine wie mich hier einzu= laden. Letztlich erfülle ich keine der an diesem Ort propagierten, angestrebten oder gar als Voraussetzung erachteten Kriterien. Ich bin Freiberuflerin, das heibt: mein.Beruf ist frei von jeder wirtschaftlichen Sicher= heit. Und dann habe ich diesen Vortrag auch noch in einer zutiefst unwissenschaftlichen Art begonnen, nämlich: persönlich. In unserer Zeit soll man schlieb= lich nichts persönlich nehmen, auch nicht die brutalste Fritik, den verletzendsten Kommentar oder den beleidig= endsten Witz. Selbstverständlich auch keine private Trennung oder berufliche Kündigung! - Nehmen Sie es ałso nicht persönlich, wenn Sie sich Miete, Heizung, Strom oder Essen nicht mehr leisten können! Nehmen Sie es nicht persönlich, wenn Ihre Gesundheit aufgrund charakterlicher Schwächen, verabscheuungswürdiger Süchte oder einem allzu hohen Alter dem Staat nicht mehr ganz so viel wert ist - und bedenken Sie dabei, dass in der Medizin bestimmt nie am falschen inde ge= spart wird, denn schlieblich gibt es ja nur eins! Nehmen Sie es weiters nicht persönlich, wenn Ihnen im Heim plätzlich Wildfremde mit akzentschwerer BabJ= sprache den Hintern abwischen, die Bank Ihnen ab einer gewissen Anzahl zur Strecke gebrachter Irbens= jahre keinen Kredit mehr gibt, oder kostbares Titan für झrsatzgelenke ebenso eher den jüngeren Steuer= zahlern zusteht wie lebenswichtige Spenderorgane! Nehmen Sie es übrigens auch nicht persönlich, dass dieser Text nicht gegendert wurde, was ich mit meiner literarisch-künstlerischen Freiheit rechtfertige sowie 
mit meiner persönlichen Abneigung gegen das Binnen-I, das in meinen Augen nur wie ein Phallussymbol aus dem Text herausragt. Aber Sie nehmen es ja gewiss auch nicht persőlich, wenn Sie von eigentlich hochkompetenten Zuständigen stets nur mangelhaft oder unverständlich informiert werden, oder wenn Behörden und Einrichtungen aller Art Ihnen Formulare zum Ausfüllen und Unterschreiben zuschicken, die Sie nicht verstehen. Nebmen Sie es deshalb auch nicht persönlich, wenn Ihre Sprache nicht mehr ge= hört und zwischen den Zeilen Thres schweigens nicht mehr gelesen wird.

Ich persönlich finde, es gibt nichts Wichtigeres, als endlich-wieder persönlich zu werden! Schonungslos per= sönlich. Hinter jeder Wirtschaftsstatistik, jeder auf Zahlen gestützten Studie, jeder Quote stehen echte Menschen. Persönliche Personen. Jedes verschriftlichte Ergebnis gründet auf einem real empfundenen Erlebnis.

Wie oft liest man in Interviews mit Prominenten, pri= vate liragen seien verboten, nur über Berufliches dürfe gesprochen werden. Mich jedoch interessiert nicht so sehr, wie ein Autor das Material für sein jüngstes Buch gefunden hat, oder wie es einem Schauspieler gelang, in eine schwierige Rolle zu schlüpfen, oder wie ein Indusirie= magnat ein Unternehmen zu Weltgeltung führen konnte, gondern der Mensch dahinter. Das zutiefst Persönliche. Worüber sich jemand freut oder kränkt, was ihn zum Heinen bringt oder lachen macht, welche Frinnerungen ihn prägen, welche Sehnsïchte ihn begleiten, welche Hoffnung ihn in die zukunft trägt. Mich interessiert, was Menschen glücklich macht, worauf sie sich freuen, wovor sie sich fürchten, wie sie mit ihren Einsamkeiten, Enttauschungen, Verlusten umgehen. Ich; als Nicht-Schauspielerin, kann von einem noch so tollen Schauspieler nichts Fachliches lernen. Aber ich kann von jedem Menschen etwas Mensch= liches lernen. Ich kann dadurch mutiger, tapferer, froher 
werden, kann mich orientieren am Umgang anderer mit Tod und Trauer, Niederlagen und Misserfolgen, Kränkung und Sorge. Mich interessieren alle Menschen dort, wo sie eben Menschen sind-nicht Firmenchefs, Verkaufsleiter, Privatdozenten, Wirtschaftsprïfer oder Spitalsvorstände, oder auch Bodybuilder, Bäcker, Bauer. Jeder einzelne Mensch ist dann nämlich interessant - weil man ihn nie ausrechnen, komplett kennen, nahtlos einschätzen kann. Jeder einzelne Mensch ist ambivalent, unvorhesehbar, un= berechenbar, vielschichtig und überraschend -wenn man ihn lässt und sich dafür interessiert. Wir haben alle mehr Gesichter als dieses eine, das wir ständig aller Welt optisch zur Verfügung stellen mijssen. Und wann ist ein Chef ein guter Chef, ein Lehrer ein guter Lehrer, ein Arzt ein guter Arzt? Wir sagen Wenn er ein Mensch ist. "Mensch geblieben", das ist durchaus ein Kompliment, wenn man es über hohe Führungskräfte, einflussreiche Politiker oder bedeutende Intellektuelle sagt.

Aber: Menschsein ist mindestens so anstrengend wie Leben! Jeder Mensch bleibt ewig Schüler, seine Schule bleibt das teben selbst. Und dieses treben bringt uns schonungslos bei, zu spät allerdings!, was wir schon zu Schulzeiten hätten wissen müssen: dass man das stete Leistungs-, Erfolgs - und Wettbewerbsdenken nicht mit ein paar Jährchen Kuschelpädagogik wegleugnen kann. Dass nicht für jedes besondere Bedürfnis eines Einzelnen eigene Assistenten und adäquate Förderungen bereitgestellt werden. Und dass alles Nachteilige- das Irren, Scheitern und Versagen - von der Gesellschaft sehr wohl persönlich genommen werden; nur nicht das spätere Darunterleiden, Daranverzweifeln oder Darüberzerbrechen.

In der Gegenwart wird das Persönliche nicht schlicht angenommen, sondern zuvor schon analysiert, systemisiert, katalogisiert und so schnell wie möglich therapiert. 
Wer feig ist, soll ein Niutseminar besuchen. Wer nur grübelt, dem empfiehlt man die Teilnahme am Lachyoga. Tier ewig trauert, wird in die Selbsthilfegruppe ge= schickt, wer allein nichts auf die Reihe kriegt, bucht einen Coach, und wer der Digitalisierung misstraut, kriegt einen Computerkurs verordnet. Aber was ist falsch oder verkehrt daran, etwas nicht zu wollen, nicht zu können, oder Unschaffbares eben nicht zu schaffen? Darf jemand nicht jahrelang um geliebte Tote trauern, weil er dadurch mit seinem Trübsinn unsere Spaßgesellschaft stört? Und warum dürfen Sterbliche nicht Laster, Süchte, Mängel haben, die ihnen kurzfristig dabei helfen, sich über ebendiese Sterblichkeit hinwegzutrösten, wenngleich auf kontra= produktive Weise?

Warum miissen immer alle erfolgreich, gesund, schön, glücklich und stark sein -oder wenigstens so wirken? Einen - noch dazu meist falschen - Eindruck erwecken zu müssen, erzeugt in erster Linie Druck auf sich selber, Druck nach innen, der die Seele deformiert, die Herven dünn scheuert, das Gemüt zerquetscht.

Ich möchte in diesem Vortrag persönlich bleiben. Pür mich war es zu keinem Zeitpunkt meines Lebens eine Frage, ob ich in der Öffentlichkeit zu viel preis= gebe. Etwa als Schriftstellerin. Denn soviel man auch schreibt, schreibt man doch niemals alles. Man lässt vieles ungesagt, um andere zu schonen, niemanden $\mathrm{zu}$ verletzen-auber vielleicht sich selbst. Offenheit im Schreiben ist die einzig logische Flucht nach vorn. Erstens, weil man nach hinten eh nicht flüchten kann, und zweitens, weil alles, was man selber zugibt, kein anderer mehr gegen einen verwenden kann. Was ich darüber hinaus noch überspitze, polemisiere oder auch kritisiere, meine ich zwar so, nur: Nehmen Sie es halt nicht persönlich! 


\section{Bildung: Wenn Dinosaurier sich erinnern}

Fines vorneweg: Dieser Vortrag entstand auf einer viele Jahrzehnte alten Schreibmaschine. Hles, was ich je geschrieben habe, entstand auf ihr. Ich fürchte, bald kommt der Tag, an dem ich die Farbbänder dafür selbst werde herstellen müssen, vielleicht aus alten Hemden, wer weiß. Altes Zeug liegt bei mir viel herum, ich werde schon was finden.

Mit neuem Zeug habe ich es nicht so. Ich bin sehr gern world wide weg von jeglicher Digitalisierung, hatte noch nie Heimwehwehweh danach. Tonnen von Weltliteratur zeugen duron, dass man auch ohne Computer Bücher schreiben kann. Heute haben alle einen Computer, nur die wenigsten schreiben Bücher damit. Ich kann mir MFIN Leben mit einem Computer nicht vorstellen, es wäre für mich unbe= wältigbar. Ich bin aus der Zeit gefallen, ich weig, bin langsam und stur, aber eben auch nicht multitaskingfähig, dafür nur begrenzt belastbar und ohnehin ziemlich schnell überfordert. Weil ich als Autorin mein eigener Chef bin, darf ich das sagen, ohne dass mich wer schimpft, feuert oder in ein entsprechendes Seminar schickt. Ich habe grundsätzlich etwas dagegen, alles mitzumachen, was alle mitmachen. In einer ielt, in der so viele unreflektiert konsumieren und besitzen, was Markt, Trend und Zeit eben so hergeben, liegt mein gröBter Luxus in der Ver= weigerung und im Verzicht auf so manches, was jetzt angeblich alle haben, machen, können, müssen, sind. Das ist meine Absage ans ingesagte. Zu meiner Unter= stützung hat Hermann Bahr schon-vor langem geschrieben: " Wahre Bildung besteht darin, zu wissen, was man kann, und ein für alle Mal zu lassen, was man nicht kann." Womit wir beim Thema wären. 
Ich bin in jede Art von Schule gern gegangen. Volks= schule, Gymnasium, Universität, ich habe das Iernen stets wie kaum etwas anderes genossen und geliebt. Für mich eine schwierige, unangepasste, depressive, zornige Jugendliche - war Bildung vor allem eines: Freiheit.

Manche meiner Gedanken zur eigenen Schulzeit passen gar nicht mehr in die heutige Realität. Ich weis gar nicht, ob ich öffentlich überhaupt noch sagen darf, wie sehr ich den Frontalunterricht immer geschätzt habe? Ist das politisch-noch korrekt? Oder grenzt das schon an Wiederbetätigung? Darf ich sagen, wie schön ich das fand, wenn da so eine fähige, charismatische und für ihre Sache wahrhaft brennende tehrperson mir etwas beibrachte, Ton dem ich noch keine Ahnung hatte? Und wie gerne ich immer mitgeschrieben habe! Mein mobilster und-effirient= ester Datenträger ist und bleibt der Bleistift. Dazu tröstet mich die geschätzte Frau Ebner ischenbach mit ihrem Zitat: "llodern sein heiBt auf dem Weg sein, un= modern zu werden."

$\therefore$.. Ich frage mich nur mehr heimlich; wann der Frontal= unterricht so in Verruf geraten ist. Wann das begonnen hat, dass plötzlich alles zum Projekt mit WorkshopCharakter-werden muss; zur unübersichtlichen Gruppen= arbeit mit Sitzkreis und ziemlich verlogenem Objektivi= täts-und-Gleichheitsmantra! Schon klar: Alle Menschen sind "gleieh, nur: jedes Hind ist anders... Seit wann genau dïrfen Jehrer nicht einfach Lehrer sein, sondern solłen zu weichgespülten "Jernbegleitern" und komplizen= haft zummengestutzten "Forschungspartnern" mutieren?

Zugegeben: Ich bin 47 Jahre alt und ein BildungsDinosaurier. Grob geworden mit Schulen, die noch B-Züge fübrten, oder überhaupt eine "Sonderschule". Statt ADHS saggte man damals noch "Nigl", "Krätzen" oder "Rotzbua". 
Dass ich mit dem heutigen Bildungssystem so meine Probleme habe, schiebe ich einer neuen Berufsgruppe in die Schuhe: den sogenannten Bildungsexperten. Was genau sind das eigentiich für Experten? Um sich etwa mit Kindern auszukennen; muss man noch nicht einmal eigene haben, meine ich. Es genügt vollends, selbst einmal Kind gewesen zu sein! Nehr Expertentum ist praktisch nicht möglich. Sich daran auch noch zu er= innern, beschert fast schon eine zusatzqualifikation. Und übrigens: Die meisten Erwachsenen kommen als Kinder zur Welt!

Es ist eine Welt, in der es statt um Wissen neuer= dings um Kompetenzen geht. Das Wort Kompetenz stammt ałlerdings nicht ursprünglich aus der Pädagogik, sondern aus der Wirtschaft. Da passt es auch hin. Denn Bildung und Ausbildung sind immer noch zweierlei! Auch Bildung, die später nicht Karriere oder Wohl= stand dient, hat einen Wert! Sogar im allerbanalsten Alltag. Denn so wenig wie "Geiz geil" ist, ist es Unbildung. Nichts ist so dermaken unsexy und $a b=$ törnend in der Begegnung der Geschlechter wie Dummheit! Dass es im wirtschaftlichen Wettbewerb natürlich oft auch um Cleverness statt Klugheit und Schläue statt Tissen geht, ist offensichtlich. Dennoch ist ein großer Feind der Bildung die zeitgeistlose Devise, dass nurr "Verwertbares" auch einen Wert hat. "Ein Bill dungsbegriff, der sich ganz an der Idee des Nütz= lichen orientiert", warnt der Philosoph Konrad Paul Liessmann deshalb nicht ohne Grund, "vergisst, dass Menschsein mehr bedeutet, als beschäftigungsfähig zu sein."

Es ist wichtig, Dinge auch um ihrer selbst willen wahrzunehmen! Leider laș̌en sich Genuss, Unterhaltung, 
Hingabe, Stil, Achtsamkeit und Sinn für Schönheit um der Schönheit willen nicht so einfach unterrichten wie Physik oder Rechnungswesen. Áber als gleichsam mystische Tugenden gehören sie zum Geheimnis des Menschseins dazu. Neugierde, Interesse und Freude zu wecken ist schlieblich ein ganz wesentlicher Bildungs= auftrag. Jeder Pädagoge, der für sein Fach brennt, ist automatisch ein guter Pädagoge. "Bildung", soll Heraklit aus Ephesos gesagt haben," ist nicht das Befüllen von Fässer.n, sondern das intzünden von Flammen."

Dass ich für Literatur brenne, verdanke ich einer büchersüchtigen und lesegierigen Familie und - tollen Lehrern und Professoren. Bücher sind für mich über= lebenswichtig. Und immer noch empfinde ich es als ge= spenstisch, eine fremde Wohnung zu betreten, in der es kein einziges sichtbares Buch gibt, dafür aber an alłen Ecken und Enden Tablets, Smartphones, Laptops und riesige Flachbildfernseher.

Ich weik, ein Computer hat für viele Menschen viele Vorteile. Es ist mir nur lieber, wenn keiner in meiner Nähe ist. Digitales ist mir unheimlich, weil es nicht greifbar ist. Da lässt sich nichts ausschneiden, $a b=$ heften und einordnen -drei meiner Lieblingsbeschäftig= ungen. Wenn ich an Literatur denke, dann an Bïcher und Zeitschriften, so wie ich bei Mrusik an Alben denke, und bei Filmen an DVD-Hüllen. Ich brauche überall ein Cover, eine-Vorder-und Rückseite, einen Anfang und ein Finde, also auch einen úberschaubaren Inhalt anstelle einer endlos in alle Richtungen ausschwappenden luieder,Text-; Informations- oder Datenflut.

Die Cnade meiner frühen Geburt erlaubt mir zudem, dass ich noch einer Sache misstrauen darf, die heute scheinbar überall gang und gebe ist: die "Power-Point- 
präsentation" und-ihre Verwandten. Dabei geht es, nicht immer!, aber allzu oft, “um souverën wirkende Đar= stellung und sonst-richt viel. Gern wird dabei hohe technische Versiertheit, gepaart mit politisch kor= rektem Sprechen und sorgsam zusammengestelltem Material schon als Paradebeispiel perfekter Bildung und, schlimmer noch, idealer Wissensvermittlung verkauft. Und genau da, wo es nur mehr um Technik statt Substanz geht, hat die Digitalfahrt gegenwärtiger Bildungsformen gleichsam freie Bahn.

-... Man muss zugeben: ein Smartphone stellt trefflich dieses fłüchtige Wisch \& Weg - Schicksal jeglicher Information dar. Wissen wird kurz und achtlos herbeiund wieder weitergestreichelt. Das ist die maximale Zärtlichkeit des nüchternen Gerätealltags. Trotzdem gibt es in der Steiermark rund 100000 "funktionale Analphabeten", die nicht sinnerfassend lesen können. Benen nützt ein technisches wunderwerk, das selbst= tätig schreibt, rechnet, verbessert undi vorliest, sicher ungemein. Nur gescheiter macht es eben dennoch nicht...

$\therefore$ Ich finde die Möglichkeit, im Internet Zugriff zu haben auf eine-schier unendliche Datenmasse und Informationsfülle, deren Quellen zum Teil undurch= schaubar bleiben, sehr verwirrend und nicht ganz un= gefährlich. Damit stehe ich freilich sofort als heiłzos reaktionärer Trottel (gegendert eventuell: Trottelin, oder-poetischer: Trottella) da, noch dazu, wenn ich zaghaft anmerken möchte, dass dabei auch so altmodische Herte wie Disziplin, Rücksicht oder Respekt klammheimlich verlorengehen in den abgrundtiefen Digitalsohlen des Schein-, Halb- und Sinnloswissens. Ich behaupte sogar, nichts hat das Hsoziale so sehr 
begünstigt wie die sogenannten "sozialen" Medien. Die bequeme Anonymität im Netz hat Indiskretion, Diffamier= ung, Respektlosigkeit und ungefilterten Hass ermöglicht und gefördert wie nichts zuvor. Wie auch kein heilsames Korrektiv der Verzögerung spontanem Unsinnverbreiten Dinhalt gebieten könnte.

Dass Kinder, und bei ihnen beginnt nun einmal jede Form der Bildung, nicht zu frîh mit Digitalität kon= frontiert werden sollen, steht jedenfalls fest, da die Reifung des Gehirns und die Entwicklung von kog= nitivem Potenzial durch den allzu frïhen Einsatz digitaler Medien und auch ebensolcher Eehrmittel gravierend-gestört werden. Aber in erster Linie steckt hinter.solchen "neuen Lernkulturen" wohl die wirt= schaft, und für die tut sich damit immerhin ein neuer, riesiger Absatzmarkt auf, der nicht unterschätzt werden darf.

Wivenn nun aber jedes sechste Kind bereits nahe am klassischen Burn-out-dahinschrammt, dürfte das nicht nur eine Folge moderner Kommunikationsmittel sein, sondern ist auch ein Indiz dafür, dass junge Menschenwie überhaupt alle Menschen in unserer Gesellschaft, mit den Ansprüchen an sie schwer überfordert sind. Gute lueistungen in der Schule werden natürlich er= wartet; und vielfach auch belohnt. Oder hörte man je von einem Kind, das gerade zum Trost für jeden Fünfer im Zeugnis ein Geschenk oder gar Bargeld erhielt?

$\mathrm{Ja}$, der Mensch hat zu funktionieren, Leistung zu bringen, Erfolge vorzuweisen - und sich permanent anzustrengen. Selbstoptimierung heiBt so ein Zauber= wort unserer Tage, und da gehört Bildung in all ihren Facetten zweifellos dazu. Das zeigt letztlich auch unsere Sprache: Früher gab es nur das Wort AUSBIIDUNG, denn da war die Bildung früher oder später ÄUS, meist 
nach dem Schulabgang. Jetzt heiBt es WITERBIGBUNG, weil es immer weitergeht mit dem Dazulernen bis zum Tod. Und was nicht vergessen werden darf: Bildung, mie fundiert und hochrangig sie auch immer sein mag, schlieBt letzthin nicht nur unser großes NichtWissen über sehr vieles ein, sondern mehr noch unser Wissen um unser Unvermögen und unsere Ohnmacht. Wissen heibt: immer zu wenig und zu viel zugleich zu wissen. Wissen heißt: alles kennen - die Fakten; Bilanzen, Statistiken-, und trotzdem nicht helfen, nicht ändern oder besser machen können. Wir wissen auch, dass viele große Karrieren in Musik, Sport; Medien und Showbranche ganz obne Bildung funktionieren, dass Reichtum und Ruhm keinerlei Klugheit voraussetzen, keines brillanten Universitätsabschlusses bedürfen, und dass in einer Telt der Selbstdarsteller, Ich-AGs und It-Personen keiner die nachdenklichen, tiefgründigen, zweifelnden, fragenden, suchenden und belesenen Persönlichkeiten dringend $z u$ brauchen scheint. In den Seichtgebieten einer momentan fortschreitenden Un-Kultur ist Bildung mitunter ein verzichtbares Gut geworden.

Uber Bildung zu sprechen, impliziert auch notwendiger= weise die Unterschei dung von Wissen, Wahrheit und Weis= heit. Wissen ist ohne Wissenschaft nicht möglich. Mit der Wahrheit aber sieht es schon ganz anders aus. Wahr= heit bleibt in vielen Bereichen des Menschenlebens ein subjektives, nahezu verhandelbares Gut. Denn auBerhalb wissenschaftlicher Fakten bestimmen eben auch Gefuhle unsere Weltsicht und unsere therzeugungen. Und wer Zahn= schmerzen kennt; Verliebtheit, Angst oder auch den unerschütterlichen Glauben an das Christkind, der weik: nichts ist so wahr wie ein Gefühl. Und Weisheit? Da darf man sich-getrost an William James halten, der be= fand: "iveisheit ist die Kunst zu wissen, was man über= sehen muss." 


\section{Beruf oder Berufung: Ich leiste (mir), was ich bin}

Fïr mich ist der Begriff "Berufsund Wirtschafts= pädagogik" ein ziemlich beeindruckendes Wort, in dessen mächtigem Schatten ich mich ein bisschen klein fühle. Ein bisschen sehr klein, genau genommen. Ich ver= binde dieses Institut und alles, was damit zusammen= hängt sowie alle, die dort zu tun haben, unweigerlich mit anderen beeindruckenden Begriffen wie "Karriere", "Laufbahn" oder auch FleiB, Stress und Tüchtigkeit. Fis erinnert mich auch an das moderne Wort "Work-LifeBalance", mit dem ich allerdings nur in meinem Leben nichts anfangen kann. Für mich ist Arbeit Leben und Eeben Arbeit, und zugleich fühlt sich Arbeit für mich nur selten so an; denn dazu mache ich sie zu gern. Öbwohl mein Arbeitsalltag sich von üblichen Berufs= bildern unterscheidet. Oder gerade deshalb?

Es-ist so: Ich habe zwei-Berufe, von denen man eigentlich nicht leben kann. Ich lebe aber FUt diese beiden Berufe, und erstaunlicherweise geht sich das dann alles irgendwie aus. Es sind zwei Berufe, die ich, präzise formuliert-nicht gelernt habe. Wenn man so will, sind es Berufungen, die mir zufielen, in die ich hineingewachsen bin. Zum einen natürlich das Schreiben. Seit über dreiBig Jahren bestimmt es maßgeblich mein Leben. Gelernt habe ich es auber in der Schule, und dort-im üblichen MaB, nirgends. Ich habe bewusst Philosophie und Anglistik studimrt, denn hätte ich Germanistik gewählt, wäre ich bestimmt nicht Schriftstellerin geworden oder geblieben. Is ist binderlich, ständig seine eigene Arbeit zu analysieren, anstatt sie einfach zu tun. Schreiben gilt zudem nicht 
als klassischer "Broterwerb". - Noch heute werde ich nach Lesungen oft gefragt, was ich denn eigentlich von Beruf sei...

$\therefore$ Nun kann ich sagen, dass ieh immerhin seit acht Jahren einen "Nebenberuf" habe: Heimhilfe im Grazer Vinzi-Dorf, Dauerwohnstätte für ehemals Irangreitob= dachlose, mehrheitlich unheilbar alkoholabhängig und sowohl physisch wie psychisch schwer krank. Ich liebe diese-Arbeit iber alles, und es gibt keinen Tag, an dem ich sie nicht gern mache. Brutal gesagt ist dieses VinziDorf die absolute, schäbige Rückseite der Wirt. schaftsuni, ja der Pädagogik überhaupt. Denn die Männer, die dort betreut werden, haben in ihrem Leben viel falsch gemacht. Bestenfalls könnten sie innerhalb der Brziehungswissenschaften als negative Beispiele Verwendung finden.

Es mag seltsam, ja provokant klingen, wenn ich das hier sage, aber: Ich habe mich immer zu denen hingezogen gefühlt, die etwas nicht schaffen, nicht können, nicht wollen, nicht sind. Menschen am Rand, mit Mängeln, Schwächen und Beschädigungen. Solche, wie eine florierende Wirtschaft sie nicht willkommen heiBt. Menschen, die von der Gesellschaft ausgestoßen wurden, die nichts mehr leisten können, nichts mehr vorzuweisen haben. Abhängige, die auBer durch ihre Süchte keine namhaften Steuerbeiträge mehr abliefern. Menschen, die nicht in den Idealtypus wohlständiger, vestlicher Industrienationen fallen; krank, sich selbst vernachlässigend, störrisch, grantig, traurig, bitter. Mienschen, die ganz klassisch den umgekehrten Heg einer Karriere gegangen sind. Die nicht flexibel oder belast= bar genug waren, um ihr Berufsleben und inren privaten Alltag reibungslos zu absolvieren. Menschen, die krank 
geworden sind, die viel verloren haben, Familie, ilohnung, Arbeitspłatz, nicht zuletzt eine Perspektive, eine - Koffnung, die ihnen den Heg in eine Zukunft hätte lemchten können. Menschen auch, die irgendwann keine Kraft zur Veränderung mehr aufbringen konnten, oder keinen Willen dafür; oder keinen Grund fanden, für den sich das noch lohnt.

涌as das alles mit Wirtschaft und Beruf zu tun hat? Mitunter mehr, als man meinen möchte. Denn im Vinzi= Dorf leben nicht nur Männer, die von vorneherein einen schlechten Start ins Leben hatten. Neben jenen, die bereits als Säuplinge weggelegt, ja weggeworfen wurden, als Kinder misshandelt und missbraucht, oder um ihre frühen Jahre betrogen wurden in einer inelt der Krimi= nalität, Prostitution und Iieblosigkeit; gibt es auch eine ganz, ganz andere Klientel. Männer, die einmal auf der Sonnenseite des Daseins zuhause waren, die viel Geld verdienten, Macht hatten, alles wünschenswerte besaßen - Schönheit, Wohlstand, Hasehen, Rang un Namen. fazu ein eigenes Büro oder sogar eine eigene Firma, eine Anwaltskanzlei oder eine Arztpraxis. Männer mit tollen Häusern, schnellen Autos, schönen Frauen. Männer, wie sie in unserer Gesellschaft am liebsten gesehen werden: erfolgreich; zielstrebig, smart. Wenn solche Menschen dann auf der Straße landen, oder im Gefängnis, in der Notschlafstelle, der Psych= iatrie oder eben im VinziDorf - wer genau hat da vers sagt? Nur die Betroffenen selber? Oder doch ein ganzes System? Iine Gesellschaft, die keine Gnade kennt mit denen, die ein eigenes Tempo haben, oder ein zu empfind= sames Gemüt, eine zu dünne- Haut, oder die einfach anders sind? Ist es die Schuld unfähiger Pädagogen, wenn aus manchen Kindern nichts wird? Sind es die 
überforderten Eltern, die den ersten Schaden verursacht haben? Die Bildungseinrichtungen, von der Volksschule bis zur Universität, die den ihnen Anvertrauten nicht das nötige Rüstzeug mitgegeben gaben für die.Welt da draußen? Kann man Resilienz wirklich lernen wie Töpfern oder Reiten? Muss man am Ende gar die Wirtschaft dafür verantwortlich machen, dass allen vorgegaukelt wird, mit genügend Ehrgeiz, Durchhaltevermögen und Ellbogen= technik sei alles möglich, sodass alles Käufliche ge= kauft werden kann, und alles irreichbareerreichbar ist? Genügt es schon, in unserer Gesellschaft nicht mehr mithalten zu können mit dem, was halt so üblich ist, mit dem, was scheinbar Halt gibt oder haltlos gierig macht? Und ist es uns, die wir arbeiten und damit Geld verdienen dürfen, ein lach über dem Kopf und den Kühl= schrank voller Speisen haben, denn zumutbar, jeden Penner, Spinner, Säufer, jeden Ob dachlosen, Vorbe= straften, Mittellosen PERSÖNLICH zu nehmen? Wer geht uns überhaupt was an, und warum?

Arbeit ist ein Grundbedürfnis des Menschen. Ganz abgesehen vom Geldverdienen brauchen wir das Gefühl, gebraucht zu werden, eine Áufgabe zu haben. Wir können die schöne, urclristliche Idee, dass jeder Mensch an und für sich einen wert hat, nicht wirklich leben; weil wir uns selbst nicht damit begnügen können. Dennoch ist es gefährlich, sich ausschlieblich über seine Arbeit $z u$ definieren. Ich bin, was ich leiste, darf genauso wenig gelten wie die logische Konsequenz daraus: Ich bin, was ich mir leisten kann. Und wenn die Globali= sierung-schon eines der ganz großen Schlagworte dieses Jahrtausends ist, dann sollte man auch in allem global denken, nicht nur bei Klima, Folter oder Krieg. Auch in Ausbildungs- oder Wirtschaftsfragen. 
Derzeit leben rund 7,5 Milliarden Menschen auf der Ëde. Etwa 80 Millionen kommen jedes Jahr dazu. Das sind für einen Eaien so unfassbare Zahlen, dass sich darunter kaum Konkretes vorstellen lässt. Aus diesem Grund haben Bevölkerungsstatistiker dankenswerterweise das-sogenannte "globale Dorf" entwickelt. Mi Zshlenspiel gelingt plötzlich -auch jemandem wie mir ein anschaulicher Blick auf unsere 弥elt. Der geht so: Tïrde die gesamte Menschheit in einem Dorf mit nur 100 Rinwobnern leben, wïrde sich die Bevölkerung unter anderem wie folgt zusammensetzen:

57 Asiaten, 21 Europäer, 5 Nordamerikaner, y Latein= amerikaner, 8 Afrikaner. 70 Personen wären Nicht= meiße, 30 Weiße. 6 Leute besäßen 59\% des Reichtums und kämen aus den USA. 80 Menschen wohnten arm= seligst, nur 20 halbwegs menschenwürdig. $19 \mathrm{Be}=$ wohner müssten von weniger als einem turo pro 'Pag leben. 12 Personen hätten zuwenig iasser, 70 wären Anэlphabeten, 50 unterernährt. Nur ein einziger (!!!) hätte einen Computer und nur einer (!!!) je eine Universität besucht. So.

Wären wir mit so einem Dorf einverstanden? Würde es etwas ändern, wenn wir die übrigen 99 Dorfbewohner pers sönlich kennen würden?

Vermutlich schon.

Mein pädagogischer Beitrag zur Gestaltung dieser Welt ist, wie könnte es anders sein?, eben auch nur ein sehr kleiner, sehr persönlicher. In Bil d»ngseinrichtungen, von der Volksschule bis zur brwachsenenbildung, gestalte ieh Schreibwerkstätten und Horkshops zu Iyrik und Philo= sophie. Ohne dazugehörige didaktische Ausbil dung, ohne amtlich verbriefte Befähigung, mit nichts als der rein 
subjektiven - Freude an der Materie und der tiefen Uberzeugung, dass Literatur und Philosophie das Leben in einer von rein wirtsehaftlichen Faktoren bestimmten Welt, in der nur angerechnët wird, was "sich rechnet", leichter, besser und erträglicher machen. Schreiben, Iesen, Denken, das hilft enorm beim Drüberleben über harte "ueiten. Dieses Wissen möchte ich am liebsten jedem mitgeben. Ganz besonders jungen Menschen. Ich weiB, wie schwierig Jungsein bisweilen ist, ich er= innere mich zu gut an meine eigene Jugend, an dieses Gefühl, gänzlich anders, allein, fremd und fehl am Platz zu sein, schadhaft, lästig, unverstanden, und an die Tatsache, nirgen dwo dazuzugehören. Später in meinem Leben sind fast alle Menschen, die mir sehr viel oder fast alles bedeutet haben, gestorben. Bücher haben mir geholfen, über Verluste, Kummer, Schmerz, Verzweiflung drüberzuleben. Bücher, irbeit, Glaube, die Natur und jeder, der noch da war und zu lieben blieb.

Schön ist, dass es im kreativen Bereich immer nur "persönlich" geht. Schreiben und Denken sind immer, ausnahmslos, persönlich. Es gibt auch, um idorno zu paraphrasieren, ein "richtiges Schreiben im falschen". Und wenn immer nur beste Leistungen, fehlerfreie Texte, Iupenreine Grammatik und virtuos verauswendigter Lern= stoff belohnt und gefördert werden, wird damit zugleich auch immer weitergebaut an dieser höchst zweifelhaften Gesellschaft, in der nur mehr beinharte Ieistung und rigide Angepasstheit honoriert werden, alles Indivi= duelle, Menschliche und damit Besondere, eben Persön= liche auf der Strecke bleibt. Und das ist es letztlich immer noch, was nicht ersetzbar ist. 
Wir wissen, wirtschaftlich ist jeder einzelne ersetzbar. In seiner Runktion, Tätigkeit, Zuständig= keit. Menschlich sieht es schon anders-aus. Da ist der genialste Arzt vielleicht eher zu ersetzenals seine uralte-Patientin es für ihre Familie einmal sein wird. Auch gute Pädagogen sind in Vahrheit unersetzlich. Kein Tablet, kein besser Ausgebildeter, keine Schulreform kann einen einzigen echten Menschen ersetzen, -der in seinen Schülern eine tiefe, dauernde Ieidenschaft entfacht, eine Liebe zu einem Wissensgebiet, die ihn durchs ganze Leben trägt. Sollten Sie selbst nicht zu jenen gehören, oder nie die beglückende Bekanntschaft mit so je= mandem gemacht haben, daw kränken 3ie sich nicht zu sehr und nehmen Sie das - ausnahmsweise - nicht persönlich. 


\section{Messung von Finanzkompetenz - Ergebnisse eines Symposiums}

Bärbel Fürstenau, Mandy Hommel, Manuel Förster, Andreas Kraitzek, Eveline Wuttke, Carmela Aprea, Michelle Rudeloff und Christin Siegfried

\section{Hinführung und Ziel}

In den letzten Jahren wird eine wachsende Bedeutung von Financial Literacy diskutiert, nicht zuletzt in Folge von Wirtschafts-, Finanz- und Schuldenkrisen vieler Länder, einer zunehmenden privaten Verschuldung und der Tatsache, dass aufgrund eines zunehmenden Rückzugs vieler Staaten aus sozialen Sicherungssystemen den Individuen mehr Verantwortung für finanzielle Angelegenheiten zukommt (Aprea et al. 2016). Aktuellen empirischen Studien zufolge weist in vielen Ländern ein großer Teil der Bevölkerung Defizite sogar in grundlegenden finanziellen Kenntnissen auf (OECD 2017b).

Angesichts dieser Situation ist Financial Literacy bzw. Finanzkompetenz ${ }^{1}$, d. h. die Fähigkeit, mit Geld und finanziellen Belangen angemessen umzugehen, für das tägliche Leben und für das langfristige finanzielle Wohlergehen sowohl von Individuen als auch von Volkswirtschaften essenziell. Die Förderung dieser Kompetenz kann durch formale Bildungsbemühungen oder informelle Lerngelegenheiten unterstützt werden. Voraussetzung dafür ist eine angemessene Modellierung und vor allem Messung dieser Kompetenz. Auf dieser Basis können zielgruppengerechte Interventionen gestaltet werden.

Derzeitige Messverfahren fokussieren jeweils Teile einer umfassenden Finanzkompetenz. Häufig steht die kognitive Komponente, speziell das Faktenwissen im Zentrum des Interesses, das über Multiple- oder Single-ChoiceItems mit einfachen Antwortstämmen erfragt wird. Ein Forschungsdefizit ist in Messverfahren zu sehen, die situativ verankert und/oder auf bestimmte Domänen bezogen sind, die zudem nicht-kognitive Aspekte und neben Ergebnissen auch Prozesse in den Blick nehmen.

1 Angemerkt sei, dass in diesem Beitrag teils von Finanzkompetenz, teils von Financial Literacy die Rede ist. Dies ist unterschiedlichen Zugängen und sprachlichen Präferenzen geschuldet. Im Rahmen des Symposiums werden beide Begriffe synonym verwendet. Im Sinne eines funktionalen Verständnisses (Weinert 2001, 2002) beschreiben sie hier komplexe domänenspezifische Fähigkeiten, die mit der Bewältigung von Anwendungssituationen verbunden, sowohl kognitive als auch emotional-motivationale, volitionale und soziale Aspekte umfassen und durch Lernen und Erfahrungen erweitert und vertieft werden können. 
Vor diesem Hintergrund war es Ziel eines Symposiums der Jahrestagung der Sektion Berufs- und Wirtschaftspädagogik 2019, zunächst einen Überblick über bestehende Instrumente und Möglichkeiten der Erfassung von Finanzkompetenz zu gewinnen und die Messung von Finanzkompetenz anschließend an zwei Beispielen zu verdeutlichen: Der Beitrag von Förster und Kraitzek (Abschnitt 2) gibt einen Überblick darüber, welche nationalen und internationalen Messverfahren vorhanden sind. Aufbauend darauf schließen die Autoren eine Forschungslücke, indem sie Desiderata vorhandener Messverfahren aufzeigen und indem sie ausloten, welche Richtung in Bezug auf Messung von Finanzkompetenz zukünftig eingeschlagen werden kann oder sollte. Der Beitrag von Wuttke, Siegfried und Aprea (Abschnitt 3) macht deutlich, wie verschiedene Facetten der Finanzkompetenz mit Situational Judgement Tests (SJT) an Stelle von Einfach- oder Mehrfachauswahlaufgaben erfasst werden können und wie bestimmte Gruppen (unterteilt nach Geschlecht, Bildungsund Migrationshintergrund) dabei abschneiden. Die Autorinnen schließen damit eine Lücke zur Klärung der Testergebnisse und Gruppenunterschiede in Abhängigkeit vom eingesetzten Instrument. Der Beitrag von Hommel und Fürstenau (Abschnitt 4) widmet sich dem komplexen Finanzprodukt der Baufinanzierung und zeigt auf, inwiefern informelles Lernen unter Berücksichtigung von Online-Informationen zur Entwicklung dieser produktspezifischen Finanzkompetenz beiträgt, welche kognitiven Prozesse dabei eine Rolle spielen und welche Fehlkonzepte entstehen. Die Autorinnen schließen eine Forschungslücke, da sie situationsorientiert unter Heranziehung einer authentischen Situation testen und zudem Prozessdaten (Think-aloud Audiografien) zur Erklärung der Produktdaten (Testergebnisse) heranziehen. Die Diskussion von Rudeloff (Abschnitt 5) würdigt die in den Beiträgen dargestellten Ansätze und gibt einen Ausblick auf zukünftige Forschung.

\section{Messung von Finanzkompetenz}

In der Forschung zur Financial Literacy kann zwar auf einen Bestand an existierenden Instrumenten zur Messung finanzieller Kompetenzen zurückgegriffen werden, welche aber auf Grund unterschiedlichster Schwerpunktsetzung hinsichtlich einer ganzheitlichen Kompetenzfeststellung ausbaufähig erscheinen. Differenziert nach internationalen, vorwiegend US-amerikanischen, und nationalen Instrumenten zur Erfassung von Financial Literacy soll hierzu im Folgenden ein kurzer Überblick gegeben werden, bevor Desiderata für zukünftige Testverfahren diskutiert werden. Der vorliegende Beitrag erhebt nicht den Anspruch, ein vollumfassendes Review aller bestehenden Instrumente zum Assessment von Financial Literacy zu liefern. Vielmehr wird der Fokus auf 
internationale wie nationale Instrumente aus der jüngeren Vergangenheit gelegt, die im Bereich des Schul- bzw. Bildungskontextes für junge Lernende angelegt sind und die exemplarisch den Stand der Instrumentenentwicklung verdeutlichen sowie die Weiterentwicklungen innerhalb des Symposiums betonen sollen. ${ }^{2}$

Die Herausforderungen bei der Erfassung von Financial Literacy bzw. Finanzkompetenz als latente Konstrukte liegen bereits in der Definition der Begriffe und in ihrer nationalen wie internationalen Anschlussfähigkeit begründet (s. Fußnote 1). In Anlehnung an Weinert $(2001,2002)$ werden im Folgenden auch Messverfahren exemplarisch betrachtet, die über eine Betrachtung rein kognitiver Facetten im Assessment hinausgehen und auch emotional-motivationale, volitionale und soziale Facetten berücksichtigen. Dies soll im Folgenden und ebenfalls in der angefügten tabellarischen Übersicht der Assessment-Tools näher dargestellt werden. Weiterhin gibt die angefügte Tabelle (s. Appendix) zum besseren Verständnis der Messung Aufschluss über die dimensionale Struktur der Messmodelle sowie die Art der Messverfahren.

Insbesondere in den USA haben die Entwicklung und der Einsatz von standardisierten und normierten Testinstrumenten zur Messung finanzieller Kompetenzen oder deren Facetten eine lange Tradition und waren auch lange Zeit verbreiteter als in Deutschland (Hausmann \& Retzmann 2014). Als Pionierarbeit können hierfür die vom amerikanischen Council of Economic Education (CEE) entwickelten Testbatterien genannt werden. Diese stellen auf die ebenfalls vom CEE entwickelten National Standards for Financial Literacy $a b$, die insbesondere für den Bereich persönlicher Finanzfragen sechs Inhaltsbereiche (Einkommen, Kauf von Gütern und Dienstleistungen, Sparen, Kreditnutzung, finanzielle Investitionen, Versicherungen) beschreiben und für eine fundierte Finanzbildung eines Individuums als essenziell angesehen werden (Walstad \& Rebeck 2016a; CEE 2013). Entwickelt wurden der Basic Finance Test (BFT) für die oberen Stufen der Grundschule (upper elementary) und die Einstiegsklassen der Mittelschule (lower middle school) (Walstad \& Rebeck 2016a), der Test of Financial Knowledge (TFK) für die obere Mittelstufe (upper middle school) und die Sekundarstufe I (lower high school) (Walstad \& Rebeck 2016b) sowie der Test of Financial Literacy (TFL) für die Sekundarstufe II und die Abschlussklassen (high school) (Walstad \& Rebeck 2016c). In all diesen Tests werden finanzielles Wissen und Verständnis mittels Single-Choice-Antwortformaten mit kurzen Item-Stämmen erfasst.

Während die vom CEE entwickelten Instrumente mit 35 (BFT), 40 (TFK) oder 45 Items (TFL) eine große Breite an finanziellen Inhaltsbereichen detailliert abbilden, verfolgen Lusardi und Mitchell (2011) einen anderen Ansatz.

2 Für weitere Übersichten siehe u. a. Schürkmann und Schuhen (2013) sowie Geiger, Meretz und Liening (2016). 
Basierend auf den Grundprinzipien Einfachheit, Relevanz, Kürze und Fähigkeit zur Differenzierung operationalisieren sie einen Indikator für Finanzwissen anhand nur weniger Items im Single-Choice-Format (Lusardi \& Mitchell 2011). Die Autoren differenzieren dabei zwischen Fragen zu basalen Finanzkonzepten (basic) und Fragen zu anspruchsvolleren Finanzmechanismen (sophisticated). Erstere adressieren u. a. die Rechenfähigkeit, Fachkenntnisse zu den Themenbereichen Inflation oder Zinseszinsen sowie das Wissen zu Risiken beim Aktienkauf in drei bis fünf Single-Choice-Items, während letztere weitergehende finanzielle Sachverhalte, wie z. B. Funktionsweisen von Aktienmärkten oder Volatilität, in acht Single-Choice-Items erfassen (Lusardi \& Mitchell 2009).

Im Kontext großangelegter internationaler Schulleistungsstudien wurde die Thematik der Financial Literacy erstmals 2012 auch in das Programme for International Student Assessment (PISA) aufgenommen und anhand von 40 Fragen aus den Inhaltsbereichen Geld und Transaktionen, Finanzplanung, Risiko und Belohnung sowie Finanzwelt (financial landscape) mittels Pen-andPaper-Test erfasst (OECD 2013). Die Aufgaben stellen dabei verstärkt einen Bezug zur lebensweltlichen Realität von 15-jährigen Schüler*innen her, indem mit (kurzen) Fallbeispielen Lebensereignisse oder -umstände, wie der Kauf einer Motorroller-Versicherung, aufgegriffen werden. Das Antwortformat variiert dabei zwischen auszuformulierenden Kurzantworten (constructed responses), kleineren Berechnungen sowie Auswahl- oder Ankreuzaufgaben (selected responses). Neben der Betrachtung dieser überwiegend kognitiven Facetten der Financial Literacy wurden im Nachgang an die Erhebung auch SchülerFragebögen ausgegeben, die nach nicht-kognitiven Aspekten, wie beispielsweise dem eigenen Sparverhalten oder dem Grad des Zugangs zu Geldinformationen und Finanzprodukten, fragten, um so Rückschlüsse auf das Interesse und die Erfahrung der Schüler*innen hinsichtlich Finanzfragen zu ermöglichen (OECD 2013).

Im deutschsprachigen Raum wurden in den vergangenen Jahren ebenso verstärkt Bemühungen zur Erforschung finanzieller Bildung unternommen. Hier wurden verschiedene Wege gewählt: Zum einen wurden die erwähnten Instrumente des TFL (Förster, Happ \& Molerov 2017) oder des TFK (Kraitzek, in Vorb.) für den Einsatz im deutschsprachigen Raum adaptiert und validiert. Des Weiteren wurden neue objektive Messverfahren entwickelt, welche einige der Limitationen der US-amerikanischen Instrumente überwinden. Hierzu zählen u. a. die von Rudeloff (2019) und Schürkmann (2017) entwickelten Testverfahren, bei denen die Aufgaben stärker situativ eingebunden sind. Parallel dazu gab es Anstrengungen, auch nicht-kognitive oder metakognitive Facetten der Finanzkompetenzen separat $\mathrm{zu}$ erfassen. So adaptierte beispielsweise Barry (2014) verschiedene englischsprachige Fragebögen zur Einstellung zu Geld ins Deutsche und fasste diese zu fünf neuen Rating-Skalen zusammen. Mit diesem Instrument wird u. a. abgefragt, welche subjektive Bedeutsamkeit 
ein Individuum der Geldthematik zuschreibt oder ob individuelle Macht- und Geltungsbedürfnisse durch den Besitz und den Umgang mit Geld erfüllt werden. Die von Barry entwickelten Skalen wurden u. a. auch in den Studien von Schürkmann (2017), Grohs-Müller (2018) und Rudeloff (2019) verwendet, um Einstellungen zu Geld zu erfassen, wobei das Instrument bei Grohs-Müller in einer modifizierten Version erweitert um die Faktoren „Glück durch Geld“ und „Angst und Sorgen“"(Grohs-Müller 2018, 91) zum Einsatz kommt. Bender und Barry (2013) gingen einen ähnlichen Weg, indem sie den von Breuer (1999) ins Deutsche adaptierten Trait Self Regulation Questionnaire (TSRQ) zur Erfassung der Selbstregulationsfähigkeit bei finanziellen Handlungen weiterentwickelten. Darüber hinaus wurden von Rudeloff (2019) und Grohs-Müller (2018) Bemühungen unternommen, die finanzielle Sozialisation zu erfassen. Rudeloff (2019) bezog zudem die Selbstwirksamkeit im Umgang mit Finanzen mit einer Adaption nach Jerusalem und Schwarzer (1999) in die Untersuchung ein, während Grohs-Müller (2018) noch Rating-Skalen für selbstberichtete Verhaltensweisen im Umgang mit Geld entwickelte.

Der Ansatz, nicht nur Wissen, sondern auch dessen Anwendung sowie nicht-kognitive Dispositionen, z. B. Einstellungen, Motivation oder Wertvorstellungen zu berücksichtigen, ist generell kompatibel mit einem ganzheitlichen Kompetenzverständnis, so wie es z. B. auch Weinert (2001) definiert hat (s. o.). Sofern allerdings nicht-kognitive Konstrukte in Studien überhaupt Berücksichtigung finden, erfolgt dies in der Regel in Form separat erfasster Rating-Skalen, welche einzelne Facetten, wie z. B. die Einstellung zu Geld, erfassen. Dies hat zur Folge, dass dann in der Regel kein Bezug auf einen bestimmten Inhaltsbereich finanzieller Kompetenz (z. B. Sparen, Versichern) genommen und der situative Kontext der vor- oder nachgelagerten kognitiven Kompetenzmessung nicht berücksichtigt wird. Neue Verfahren müssen kognitive und nicht-kognitive Facetten möglichst simultan im gleichen Szenario und der gleichen Situation erfassen, da dies die Validität der Konstrukte deutlich erhöhen und bessere Rückschlüsse auf deren Zusammenhänge bei der Lösung von Aufgaben ermöglichen würde.

Weiterhin herrscht bislang eine starke, wenn nicht sogar ausnahmslose Fokussierung auf die durch Tests gemessenen Kompetenzen am Ende einer Intervention oder Lerneinheit, während der eigentliche Prozess der Problemlösung in seiner Feinheit bisher kaum betrachtet wird. Aber gerade die Prozessperspektive wäre aus diagnostischer Sicht von hoher Relevanz, um Aussagen treffen zu können, welche kognitiven und nicht-kognitiven Prozesse eine Rolle spielen und in welchen Phasen des Problemlösungs- oder Entscheidungsprozesses die Proband*innen bestimmte Fehler oder Fehlkonzeptionen entwickeln.

An diesen Forschungsdesiderata setzten die folgenden Beiträge des Symposiums an. Wuttke, Siegfried und Aprea setzen erstmals einen SJT zur Erfassung von Finanzkompetenz ein. Hommel und Fürstenau untersuchen, wie sich 
die finanziellen Kompetenzen von Studierenden entwickeln, die informelle Lerngelegenheiten zur Finanzthematik im Internet wahrnehmen. Dies geschieht im konkreten Fall anhand des komplexen Finanzproduktes der Baufinanzierungsdarlehen.

\section{Messung von Financial Literacy mit einem Situational Judgement Test - sind bestimmte Personengruppen wirklich schlechter oder liegt es am Messinstrument?}

Problemstellung und konzeptuelle Grundlagen

Aktuelle Studien zu Financial Literacy zeigen u. a., dass männliche Probanden in Tests oft besser abschneiden als weibliche (z. B. Bucher-Koehnen et al. 2017), und dass Personen mit Migrationshintergrund und solche mit niedrigem Bildungsniveau oft schlechter abschneiden als solche ohne Migrationshintergrund (z. B. Happ \& Förster 2019) und/oder hohes Bildungsniveau (z. B. Lusardi \& Mitchell 2014). Diese Befunde werden häufig als Indiz für die Schwäche und den besonderen Förderbedarf der genannten Gruppen angesehen. Wie Forschungsarbeiten aus angrenzenden Gebieten wie etwa der ökonomischen Bildung (z. B. Siegfried \& Wuttke 2019) oder der Mathematik (z. B. Lindner et al. 2015; Reardon et al. 2018) sowie zahlreichen anderen Assessmentbereichen (z. B. Saygin 2019) zeigen, können solche Gruppenunterschiede jedoch (zumindest partiell) auch aus Benachteiligungen resultieren, die auf das Messinstrument zurückzuführen sind. Eine systematische Untersuchung dieser Interpretation liegt im Bereich von Financial Literacy bislang noch nicht vor, ist jedoch von zentraler Bedeutung sowohl für die wissenschaftliche Erklärung von Leistungsunterschieden als insbesondere auch für die praktische Entscheidungsfindung in Bezug auf entsprechende Bildungsmaßnahmen. Die im Folgenden vorgestellte Studie möchte einen Beitrag zur Schließung dieser Forschungslücke leisten, indem ein alternativer Zugang zur Messung von Financial Literacy vorgestellt und eruiert wird, ob und inwieweit dieser Zugang zu Abweichungen von bisherigen Ergebnissen führt. Die Studie ist Bestandteil eines breit angelegten Forschungsprogramms zur Entwicklung eines validen Assessments von Financial Literacy.

Der alternative Zugang basiert in Anlehnung an Ansätze aus der Wirtschaftsdidaktik auf einer kompetenzorientierten Konzeptualisierung von Financial Literacy (Aprea \& Wuttke, 2016; Wuttke \& Aprea, 2018). Zur Entwicklung entsprechender Kompetenzfacetten wurden zunächst Interviews mit 40 internationalen Experten aus dem Bildungs- und Finanzbereich geführt (Aprea et al. 2015). Es wurden 9 Facetten ermittelt, für die Testitems gemäß 
dem Ansatz des SJT (McDaniel et al. 2007) konstruiert wurden. Dieser Ansatz wurde gewählt, da die Messung von Financial Literacy im Sinne eines ganzheitlichen Konstruktes einen Messzugang erfordert, der in der Lage ist, komplexe Fähigkeiten zu erfassen und diese durch ein Set an Aufgaben beobachtbar zu machen, deren Anforderungen möglichst nahe an der erwarteten Performanz liegen. Solche SJT präsentieren realistische, hypothetische Situationen, zu denen sich die Probanden ,angemessen“ verhalten müssen. Bisherige Befunde zeigen, dass mit dieser Art der Testung Verhalten in Echtsituationen gut vorhergesagt werden kann (Kahmann 2014).

\section{Forschungsfragen}

Die Frage, die sich v. a. mit Blick auf die neue Testkonzeption stellt, ist, ob sich in den verschiedenen Gruppen die Ausprägung von Financial Literacy unterscheidet (wie es bisherige Befunde nahelegen), oder ob von Unterschieden auf Basis des eingesetzten Instruments, also einem Testbias auszugehen ist. Deshalb wird in einem ersten Schritt geprüft, ob ein latenter Mittelwertvergleich der Gruppen (weibliche vs. männliche Testpersonen, Personen mit oder ohne Migrationshintergrund und Personen mit akademischen vs. beruflichem Bildungshintergrund) zulässig ist. Hierfür ist zunächst zu prüfen, ob für die zu untersuchenden Gruppen Messinvarianz angenommen werden kann. Sollte das der Fall sein, werden Gruppenunterschiede geprüft.

\section{Untersuchungsdesign}

Es wurden insgesamt 22 Items zu den Kompetenzfacetten „Kontrolle der eigenen finanziellen Situation“, „Budgetierung“ und „Umgang mit Geld“ eingesetzt. Die Skalen haben zufriedenstellende Reliabilitäten (Kontrolle der eigenen finanziellen Situation, 9 Items, max. 36 Punkte, $\alpha=.754$; Budgetierung, 6 Items, max. 24 Punkte, $\alpha=.573$; Umgang mit Geld 7 Items, max. 28 Punkte, $\alpha=.691$ ). An der Erhebung nahmen 206 junge Erwachsene teil (weiblich 52,2\%; Altersdurchschnitt 20,06; Bildungshintergrund: 105 Studierende, 101 berufliche Ausbildung (Vollzeitschulen und duale Ausbildung), 62\% mit Migrationshintergrund $=$ Muttersprache beider Eltern ist nicht Deutsch).

Die Daten wurden mit Blick auf das Geschlecht, den Bildungshintergrund und den Migrationshintergrund Mittelwertvergleichen unterzogen. Vor Überprüfung von Mittelwertunterschieden werden folgenden Messinvarianzmodelle (mit zunehmender Restriktion) geprüft: Konfigurale Messinvarianz, metrische Invarianz, skalare Invarianz, strikte Invarianz/Invarianz der Messfehler (Temme \& Hildebrand 2008). 
Ergebnisse

I Geschlecht: Im Rahmen der konfiguralen Invarianzmodelle zu den drei Facetten von Financial Literacy wurden alle Faktorladungen, Intercepts und Residualvarianzen über beide Geschlechter hinweg frei gesch tzt. Die Fit-Statistiken zeigen zunächst, dass der Modelfit bei allen drei konfiguralen Invarianzmodellen angenommen werden kann (,Kontrolle“: $\chi 2=61.07, \mathrm{p}=.098, \mathrm{df}=$ $48, \mathrm{CFI}=.95 ; \mathrm{RMSEA}=.037$, Pclose $=.801 ;$,Budgetierung““ $: \chi^{2}=41.103, \mathrm{p}$ $=.008, \mathrm{df}=22, \mathrm{CFI}=.94 ; \mathrm{RMSEA}=.066$, Pclose $=.185 ;$, Umgang mit Geld“ $\chi 2=36.555, \mathrm{p}=.006, \mathrm{df}=18, \mathrm{CFI}=.89 ; \mathrm{RMSEA}=.072$, Pclose $=.131$ ), auch wenn der CFI für die Facette „Umgang mit Geld“ zu niedrig ausfällt. Damit kann angenommen werden, dass die drei Faktoren in beiden Gruppen in hnlicher Weise konzeptualisiert sind. Hinsichtlich der Gleichheitsrestriktionen auf den Faktorladungen, die im Rahmen der metrischen Invarianz gesetzt wurden, verhält es sich allerdings je nach Facette unterschiedlich. Während sich für die Facette „Kontrolle der eigenen finanziellen Situation“ und „Budgetierung“ die Modellpassung nicht bedeutsam verschlechtert $(\triangle \mathrm{CFI} \leq$. |.02|, $\triangle$ RMSEA $\leq$ .015) und somit abgeleitet werden kann, dass die Maßeinheit der beiden Skalen für weibliche und männliche Testteilnehmer identisch ist, stellt sich für die Facette „Umgang mit Geld“ bei dieser Modellrestriktion eine sich weiter verschlechternde Modellpassung heraus $(\chi 2=47.516, \mathrm{p}=.003, \mathrm{df}=24, \mathrm{CFI}=$ .86 ; RMSEA $=.07$, Pclose $=.122$ ). Erst durch die Freisetzung der Faktorladung eines Items (dies fand auf Basis der Größe des Modifikationsindexes statt) kann ein ähnlicher Modelfit wie bei der konfiguralen Messinvarianz erreicht werden $(\chi 2=43.184, \mathrm{p}=.007, \mathrm{df}=23, \mathrm{CFI}=.89$; RMSEA $=.066$, Pclose $=.175)$.

Für die Prüfung der starken/skalaren Messinvarianz wurden neben den Faktorladungen auch die Intercepts der einzelnen für die latenten Skalen herangezogenen Items für männliche und weibliche Probanden gleichgesetzt. Hierbei wird durch die Verschlechterung der Modellpassungen $(\triangle \mathrm{CFI}>.|.02|)$ deutlich, dass für keine der Facetten diese Modellrestriktion angenommen werden kann. Auch hier lohnt es sich allerdings zu untersuchen, ob die starke Invarianz zumindest partiell erreicht werden kann. Hierfür wurden für die Facette „Kontrolle der eigenen finanziellen Situation“, „Budgetierung“ und „Umgang mit Geld“ " je ein Intercept freigesetzt, während die anderen Faktorladungen auf Gleichheit über die beiden angenommenen Subgruppen restringiert wurden. Die Teststatistiken des Modellvergleichs zeigen, dass durch diesen Zugang partielle Messinvarianz für die beiden Facetten ,Kontrolle der eigenen finanziellen Situation“ $(\chi 2=86.701, \mathrm{p}=.002, \mathrm{df}=65, \mathrm{CFI}=.91$; RMSEA $=.041$, Pclose $=.736)$ und "Budgetierung" $(\chi 2=59.521, \mathrm{p}=.006, \mathrm{df}=35, \mathrm{CFI}=.92$; RMSEA $=.059$, Pclose $=.259)$ erreicht werden konnte. Für die Facette „Umgang mit Geld" ist die Modellpassung allerdings nicht gegeben $(\chi 2=52.835$, $\mathrm{p}=.002, \mathrm{df}=27, \mathrm{CFI}=.85$; RMSEA $=.069$, Pclose $=.128)$. Für die Facette 
„Budgetierung“ zeigt sich darüber hinaus, dass sogar eine strikte Messinvarianz angenommen werden kann $(\chi 2=70.489, \mathrm{p}=.004$, df $=42, \mathrm{CFI}=.91$; RMSEA $=.058$, Pclose $=.265$ ).

Vor diesem Hintergrund ist lediglich für die Facetten „Kontrolle der eigenen finanziellen Situation“ und „Budgetierung“ die Voraussetzung für einen sinnvollen Vergleich der Mittelwerte dieser Skalen gegeben. Der T-Test zeigt in diesem Zusammenhang lediglich einen signifikanten Unterschied für die Facette „Kontrolle der eigenen finanziellen Situation“ zum Vorteil weiblicher Probanden (Kontrolle: $\mathrm{t}(182)=-3.058, \mathrm{p}=.003$; Budgetierung: $\mathrm{t}(186)=-$ $1.018, \mathrm{p}=.310)$.

Aus Platzgründen kann an dieser Stelle nicht ausführlicher auf die Analyse der Messinvarianz zu Bildungshintergrund und Migrationshintergrund eingegangen werden. Es wurde so vorgegangen, wie bei der Analyse der Messinvarianz bei männlichen/weiblichen Probanden (ausführlich Wuttke, Siegfried \& Aprea, in Vorbereitung). Zusammenfassend lässt sich festhalten:

II Bildungshintergrund: Es kann eine (partielle) starke Messinvarianz für die beiden Facetten „Kontrolle der eigenen finanziellen Situation“ und „Budgetierung“ erreicht werden. Für diese Facetten ist der Vergleich der Mittelwerte der Skalen zwischen Testpersonen mit beruflichem vs. akademischem Bildungshintergrund zulässig. T-Tests zeigen, dass Probanden, die aktuell ein Studium absolvieren in der Facette „Budgetierung“ signifikant besser abschneiden als diejenigen, die im beruflichen Schulwesen sind (Kontrolle: $\mathrm{t}(184)=-0.491, \mathrm{p}=.62$; Budgetierung: $\mathrm{t}(187)=-3.073, \mathrm{p}<.01)$.

III Migrationshintergrund: Für alle drei Facetten ist die Voraussetzung für die Testung von Mittelwertunterschieden zwischen den Subgruppen gegeben. Die Ergebnisse der T-Tests verweisen darauf, dass für die Facetten „Kontrolle der eigenen finanziellen Situation“ und „Budgetierung“ keine signifikanten Gruppenunterschiede bezüglich des Migrationshintergrundes vorliegen (Kontrolle: $\mathrm{t}(181)=-1.367, \mathrm{p}=.17$; Budgetierung: $\mathrm{t}(184)=0.817, \mathrm{p}=.42$ ), allerdings ist dies der Fall für die Facette „Umgang mit Geld“ $(\mathrm{t}(179)=-1.955$, $\mathrm{p}=.05)$ und zwar zum Vorteil für diejenigen Probanden, die keinen Migrationshintergrund aufweisen.

\section{Relevante Implikationen und Limitationen}

Die Analyse der Messinvarianz zeigt, dass der entwickelte Test für die Facetten Kontrolle der eigenen finanziellen Situation und Budget erstellen bei allen betrachteten Gruppen Messinvarianz aufweist, Gruppenvergleiche sind damit möglich und potentielle Unterschiede sind nicht auf einen Testbias zurückzuführen. Für die Facette Umgang mit Geld kann keine Messinvarianz angenommen werden. 
Entgegen der Befunde aus vielen bisherigen Studien zeigen sich bei der Analyse der Mittelwertunterschiede keine systematischen Nachteile für bestimmte Gruppen: Mit Blick auf das Geschlecht zeigt sich, dass weibliche Probanden bei der Budgetierung dieselbe Leistung zeigen wie männliche Probanden, bei der Facette Kontrolle der eigenen finanziellen Situation schneiden sie sogar signifikant besser ab. Mit Blick auf den Bildungshintergrund zeigt sich bei der Facette Budgetierung ein erwartungskonformer Vorteil für Personen mit akademischem Hintergrund, bei den übrigen Facetten sind keine Unterschiede zu finden.

Insgesamt legen die Ergebnisse damit den Schluss nahe, dass häufig die Art der Testung mit möglichen Vor- oder Nachteilen für bestimmte Gruppen verbunden ist (s. a. Greimel-Fuhrmann \& Silgoner 2018; Siegfried \& Wuttke 2019). Dies scheint im vorliegenden SJT weniger der Fall zu sein.

Die Facette Umgang mit Geld ist vor dem Hintergrund der fehlenden Messinvarianz zu überarbeiten. Hierzu ist eine Studie mit Nutzung lauten Denkens geplant, die Hinweise geben soll, wie die Items der Facette zu überarbeiten sind.

\section{Kognitive Prozesse und Fehlkonzepte beim informellen Erwerb von Finanzkompetenz über Baufinanzierung}

\section{Relevanz und Zielsetzung}

Mit dem Ziel, die Finanzkompetenz von Individuen zu fördern, haben - insbesondere seit der Finanzkrise im Jahr 2007/2008 - viele Länder entsprechende Bildungsprogramme zur Financial Education entwickelt und zum Teil curricular verankert. Dadurch sollen negative Auswirkungen potenzieller Fehlentscheidungen sowohl auf persönliche Finanzsituationen (OECD 2013) als auch auf ganze Volkswirtschaften vermieden werden. Neben diesen Bildungsprogrammen, die als formales Lernen gewertet werden können, ist es jedoch auch möglich, Finanzkompetenz informell zu erwerben. Informeller Erwerb von Finanzkompetenz stand jedoch bislang nicht im Zentrum von Forschungsbemühungen in diesem Kontext.

Insbesondere das Internet bietet eine Fülle von Informationen, die Individuen bedarfsorientiert nutzen können, um sich in einer bestimmten Domäne, z. B. bezogen auf komplexe Finanzprodukte, informell weiterzubilden (Dennen \& Wang 2002; Werquin 2010). Dieses informelle online Lernen ist insgesamt nicht so gut erforscht wie informelles Lernen im Allgemeinen. Bezogen auf die Frage, welche Designprinzipien informelles online Lernen von Erwachsenen unterstützen, zeigt eine neuere Metasynthese, dass es insbesondere 
auf Möglichkeiten zur Interaktion (Wissen und Erfahrungen zu Themen mitteilen) sowie auf gut gegliederte und markierte sowie klar betitelte Informationen ankommt. Weitere Prinzipien, wie eine möglichst umfangreiche Informationsbasis oder hohe soziale Präsenz, sind noch nicht hinreichend gesichert (Holland 2019). Auf Seiten des Individuums spielen Faktoren, wie die Fähigkeit selbstgesteuert zu lernen, vor allem aber Vorwissen und Vorerfahrung, eine Rolle (Maier et al. 2014; Cerasoli et al. 2018). Die Forschung zeigt, dass informelles online Lernen nicht immer erfolgreich ist. Es können beispielsweise Fehlkonzepte als ,inkorrektes Wissen“ (Eitel, Dersch \& Renkl 2019, 454) entstehen. Aus Verständnisproblemen und Unsicherheiten, die nicht geklärt werden, entwickeln sich solche Fehlkonzepte. Diese sind problematisch, da sich aus ihnen handlungsrelevante (aber eben fehlerhafte) Überzeugungen entwickeln können (ebd., 453).

Im Rahmen einer Studie wurde geprüft, inwiefern online verfügbare Informationen das informelle Lernen unterstützen, welche kognitiven Aktivitäten dabei eine Rolle spielen und welche Verständnisschwierigkeiten entstehen können. Konkret stand ein komplexes Finanzprodukt, in diesem Fall Baufinanzierungsdarlehen, im Zentrum des Interesses. Mit Blick auf die Messung von Finanzkompetenz greifen wir gezielt den Aspekt der Situationsbezogenheit auf, da sich die Probanden in eine komplexe Entscheidungssituation über die Aufnahme eines Baufinanzierungsdarlehens hineinversetzen und sich aus diesem Kontext heraus Wissen aneignen müssen. Weiterhin beziehen wir in die Messung sowohl Produktdaten (Testergebnisse) als auch Prozessdaten (Thinkaloud-Protokolle, die Fehlkonzepte verdeutlichen) ein, um dadurch Ergebnisse besser erklären und Anhaltspunkte für instruktionale Unterstützung gewinnen zu können.

\section{Forschungsfragen}

I. Tragen Webseiten von Banken im Rahmen informellen Lernens dazu bei, die Entwicklung von Finanzkompetenz potentieller Darlehensnehmer*innen in Bezug auf Baufinanzierungsdarlehen zu fördern?

II. Welche kognitiven Aktivitäten sind mit der Exploration von Webseiten zu Baufinanzierungen durch potentielle Darlehensnehmer*innen verbunden?

III. Welche Verständnisschwierigkeiten haben potentielle Darlehensnehmer*innen beim Explorieren eines Darlehenskalkulators? 


\section{Methode}

Die Stichprobe umfasst 45 Studierende der Wirtschaftswissenschaften (Tab. 1), die zufällig einer Experimentalgruppe (EG) oder einer Kontrollgruppe (KG) zugewiesen wurden.

Tab. 1: Stichprobe

\begin{tabular}{lccccc}
\hline & $\mathrm{n}$ & $\%$ & Alter M & Geschlecht & Think-aloud \\
\hline EG & 23 & 51.1 & 21.8 & $6 \mathrm{~m}, 17 \mathrm{w}$ & 12 \\
KG & 22 & 48.9 & 22.5 & $4 \mathrm{~m}, 16 \mathrm{w}, 2 \mathrm{~d}$ & 6 \\
Total & 45 & 100 & 22.2 & $10 \mathrm{~m}, 33 \mathrm{w}, 2 \mathrm{~d}$ & 18 \\
\hline
\end{tabular}

Quelle: eigene Abbildung

Die Proband*innen der EG explorierten die Webseite der Commerzbank zum Darlehenskalkulator (Hommel et al. 2017; Fürstenau \& Hommel 2018). Dieser Kalkulator wurde ausgewählt, da er mehr Informationen und somit potenziell mehr Lerngelegenheiten zur Verfügung stellt als die Kalkulatoren anderer Banken. Die Qualität der Informationen dieses Kalkulators ist vergleichbar mit denen der Kalkulatoren anderer Banken (Fürstenau et al. 2016). Die Proband*innen der KG hatten keinen Zugang zu (Internet-)Informationen über Baufinanzierungen. Im Rahmen einer Fallsituation mussten die Proband*innen beider Gruppen darüber entscheiden, ob sie unter den gegebenen Bedingungen ein Baufinanzierungsdarlehen aufnehmen. Die Entscheidung war zu begründen.

Vor der Exploration bearbeiteten alle Teilnehmenden einen Pretest zu numerischen Fähigkeiten (Prozent-, Zins- und Annuitätenrechnung) sowie eine Selbsteinschätzung in Bezug auf Finanzwissen zu Baufinanzierungsdarlehen. Insgesamt waren im Pretest sieben Punkte erreichbar. Nach der Exploration absolvierten alle Personen einen Posttest mit vier offenen Items, u. a. zu den Komponenten einer Annuität, dem Konzept Zinsfestschreibung etc., sowie neun Einfachwahlaufgaben zum Inhaltsbereich Baufinanzierung. Im Posttest waren maximal 21 Punkte erreichbar. Beispiele zu den Messinstrumenten finden sich bei Hommel et al. (2017) sowie Fürstenau und Hommel (2019).

Während der Exploration und bei der Bearbeitung der Fallsituation externalisierten 12 Proband*innen der EG und sechs Proband*innen der KG ihr Denken laut (Fürstenau \& Hommel 2019). Die Think-aloud-Daten wurden audiografiert, transkribiert und mithilfe einer kategorialen Inhaltsanalyse ausgewertet (Krippendorf 2012). Dazu wurde das Kategoriensystems für ,self-explanations' von Chi et al. (1989) adaptiert. Der Kodierleitfaden wurde von 
zwei Kodierenden gemeinsam entwickelt. In diesem Prozess stand die semantische Gültigkeit im Fokus, um die Bedeutung der kodierten Aussagen beizubehalten bzw. zu rekonstruieren (Mayring 2015). Die Aussagen wurden in den Hauptkategorien (1) Erklären/Elaborieren (Äußerungen zu Informationen/Fakten, denen Bedeutung zugewiesen wird, die evaluiert werden, oder zu Konsequenzen, die durchdacht werden), (2) Monitoring (Aussagen zum eigenen Lernen, wie zum Verstehen, zu Unsicherheiten im Verständnis oder zum Nichtverstehen) sowie (3) Andere (Wahrnehmen, Paraphrasieren von Informationen; Fragen zum Inhalt, metastrategische Aussagen) zugeordnet.

\section{Ergebnisse}

In den Ergebnissen des Pretests (Tab. 2) unterschieden sich die Proband*innen der $\mathrm{EG}$ und $\mathrm{KG}$ nicht signifikant $(\mathrm{n}=45, \alpha=.05, \mathrm{t}=-1.071, \mathrm{df}=43, \mathrm{p}=.290$, $\mathrm{d}_{\text {Cohen }}=0.315$, Teststärke $\left.(1-\beta)=.26\right)$. Auch im Posttest erreichten die Gruppenunterschiede nicht das Signifikanzniveau $\left(\mathrm{t}=-1.180, \mathrm{df}=43, \mathrm{p}=.245, \mathrm{~d}_{\mathrm{Co}}\right.$ hen $=0.355,(1-\beta)=.31)$. Mithilfe einer mixed ANOVA wurden der Wissensstand zu den beiden Zeitpunkten (Innersubjektfaktor) und der Zwischensubjektfaktor Gruppe gemeinsam analysiert. Allerdings zeigt sich in unserem Fall ausschließlich ein signifikanter Einfluss des Faktors Zeit $(F(1,43)=241.28$, $\mathrm{p}<.001$, partielles $\eta^{2}=.85$.) und kein Interaktionseffekt zwischen der Zeit und der Gruppe $\left(F(1,43)=0.923, p>.05\right.$, partielles $\left.\eta^{2}=.021\right)$. Insofern trägt informelles Lernen unter Nutzung von Webseiten scheinbar nicht zum Erwerb von Finanzkompetenz bei (Forschungsfrage I).

Tab. 2: Punkte im Pretest und Posttest

\begin{tabular}{ccccccccc}
\hline & \multicolumn{4}{c}{ KG } & \multicolumn{5}{c}{ EG } \\
& M & SD & Min & Max & M & SD & Min & Max \\
\hline Pretest & 3.00 & 1.45 & 0 & 6 & 3.43 & 1.27 & 1 & 6 \\
Posttest & 11.18 & 3.74 & 3 & 17 & 12.70 & 4.78 & 4 & 21 \\
\hline
\end{tabular}

Quelle: eigene Abbildung 
Um Anhaltspunkte dafür zu gewinnen, warum dies der Fall ist, wurden die kognitiven Prozesse analysiert (Forschungsfrage II) und die Think-aloud-Daten der Proband*innen der EG denen der KG gegenübergestellt (Tab. 3).

\section{Tab. 3: Kategorien der Think-aloud-Kodierung}

Think-aloud-Kategorie

(1) Erklären/Elaborieren

\begin{tabular}{cccc}
\multicolumn{2}{c}{ KG } & \multicolumn{2}{c}{ EG } \\
\hline $\mathrm{n}$ & $\%$ & $\mathrm{n}$ & $\%$ \\
\hline 236 & 49.2 & 551 & 34.1
\end{tabular}

Evaluieren von Fakten, Ergebnissen, Informationen

$\begin{array}{llll}30 & 6.3 & 133 & 8.2\end{array}$

Erklären oder Ableiten von Konsequenzen

$\begin{array}{llll}188 & 39.2 & 289 & 17.9\end{array}$

Zuweisen von Bedeutungen zu Aussagen

Definieren, Verfeinern, Filtern oder Erweitern von Bedingungen, die Entscheidung beeinflussen

\begin{tabular}{lcccc}
\hline (2) Monitoring & 47 & 9.8 & 112 & 6.9 \\
Aussagen über Nichtverstehen & 33 & 6.9 & 58 & 3.6 \\
Aussagen zu Unsicherheiten im Verstehen & 9 & 1.9 & 28 & 1.7 \\
Aussagen zum Verstehen von Informationen & 5 & 1.0 & 26 & 1.6 \\
\hline (3) Andere & 197 & 41 & 952 & 59 \\
Auswählen aus einem Drop-down-Menü & 18 & 3.8 & 136 & 8.4 \\
Metastrategische Aussagen & 35 & 7.3 & 217 & 13.4 \\
Paraphrasieren gegebener Informationen & 29 & 6.0 & 118 & 7.3 \\
Wahrnehmen oder Erinnern von Informationen & 11 & 2.3 & 93 & 5.8 \\
Fragen zum Inhalt & 16 & 3.3 & 157 & 9.7 \\
Lesen von Informationen & 88 & 18.3 & 231 & 14.3 \\
\hline Total & 480 & 100 & 1615 & 100 \\
\hline
\end{tabular}

Quelle: eigene Abbildung

Im Vergleich der Häufigkeiten der Think-aloud-Kodierungen wird deutlich, dass der relative Anteil von Aussagen in der Kategorie Erklären/Elaborieren mit 49.2\% in der Kontrollgruppe (KG) über dem der Experimentalgruppe (EG) mit 34.1\% der jeweils kodierten Aussagen liegt. Innerhalb dieser Kategorie entfallen $39.2 \%$ in der KG und nur $17.9 \%$ in der EG auf das Erklären/Ableiten 
von Konsequenzen. Auch in der Kategorie Monitoring liegt der relative Anteil der Aussagen in der KG mit 9.8\% über dem der EG mit 6.9\%. Dagegen zeigt sich der überwiegende Anteil der kodierten Aussagen in der EG mit 59\% in der Kategorie Andere im Vergleich zur KG mit 41.0\%. In der EG beziehen sich diese Aussagen mit 8.4\% auf das Auswählen von Informationen, z. B. aus Drop-down-Menüs des Kalkulators, und mit 13.4\% auf metastrategische Aspekte, wie das Reflektieren bereits vollzogener Schritte, deren Reihenfolge oder noch auszuführende Aktionen. Im Vergleich der Verteilung der Kodierungen in den Hauptkategorien wird deutlich, dass die Probanden der EG stärker damit beschäftigt sind, sich zu orientieren als Inhalte in ihrer Bedeutung zu erfassen und zu elaborieren. Der Kalkulator der Commerzbank erfordert dementsprechend offenbar viele Orientierungsprozesse, die zu Lasten essentieller Lernprozesse gehen können.

Im Rahmen der Think-aloud-Kodierungen wurden die Aussagen zum Nichtverstehen bzw. zu Unsicherheiten einer ergänzenden inhaltlichen Analyse unterzogen (Forschungsfrage III). Die hier erfassten Kodierungen der Aussagen liefern Hinweise auf mögliche Fehlkonzepte und Fehler in der Informationsverarbeitung, die Fehlkonzepte begünstigen (Tab. 4).

Verständnisprobleme konnten insbesondere für die Konzepte Annuität, Zinsfestschreibung, anfängliche jährliche Tilgung, Bereitstellungszins, Eigenkapital, Eigentum und Wohneinheit identifiziert werden. Obwohl für diese Konzepte teils Erläuterungen im Darlehenskalkulator hinterlegt sind, verstehen nicht alle Proband*innen der EG deren Bedeutung korrekt. Unklarheiten entstanden auch hinsichtlich der Ermittlung des Darlehensbetrags. Daneben greifen Proband*innen, anstatt die Annuitätenrechnung als Verfahren zu nutzen, auf überschlägige Näherungsrechnungen zurück. Als Fehlkonzepte sind insbesondere der Bereitstellungszins, die Wohneinheiten und der anfängliche jährliche Tilgungssatz zu identifizieren. So wird z. B. der Bereitstellungszins als Strafzins für Sondertilgungen verstanden, die Wohneinheit mit der Anzahl der Räume gleichgesetzt und die anfängliche jährliche Tilgung als zusätzlich zur Annuität aufzubringender Tilgungsbetrag interpretiert. 
Tab. 4: Fehlkonzepte

\begin{tabular}{lllll}
\hline Fehlkonzepte & $\begin{array}{l}\text { Häufigkeit } \\
\text { absolut }\end{array}$ & $\begin{array}{l}\text { Häufigkeit } \\
\text { relativ }\end{array}$ & $\begin{array}{l}\text { KG } \\
(\mathrm{n}=6)\end{array}$ & $\begin{array}{l}\text { EG } \\
(\mathrm{n}=12)\end{array}$ \\
\hline $\begin{array}{l}\text { Annuität (Zins und } \\
\text { Tilgung) }\end{array}$ & 10 & 16.67 & 7 & 3 \\
$\begin{array}{l}\text { Darlehensbetrag } \\
\begin{array}{l}\text { Zinsfestschreibung } \\
\text { (Zinsbindung) }\end{array}\end{array}$ & 10 & 16.67 & 3 & 7 \\
$\begin{array}{l}\text { Bereitstellungszins } \\
\text { (-freie Zeit) }\end{array}$ & 5 & 11.67 & 2 & 5 \\
Näherungsrechnung & 5 & 8.33 & 0 & 5 \\
$\begin{array}{l}\text { Prozentrechnungs- } \\
\text { fehler }\end{array}$ & 5 & 8.33 & 5 & 0 \\
Wohneinheit & 5 & 8.33 & 4 & 1 \\
Eigentum & 4 & 8.33 & 0 & 5 \\
$\begin{array}{l}\text { Anfängliche jährliche } \\
\text { Tilgung }\end{array}$ & 2 & 6.67 & 0 & 4 \\
Bauart & 2 & 3.33 & 1 & 1 \\
Eigenkapital & 2 & 3.33 & 0 & 2 \\
Erbbaurecht & 1 & 3.33 & 2 & 0 \\
Frei verfügbares & 1 & 1.67 & 0 & 1 \\
Einkommen & 1 & 1.67 & 0 & 1 \\
Nebenkosten & 60 & 1.67 & 0 & 1 \\
Summe & & & 24 & 36 \\
\hline & & & & \\
\hline
\end{tabular}

Quelle: eigene Abbildung

Konsequenzen für die Förderung von Finanzkompetenz

Die Ergebnisse der Studie zeigen, dass das Explorieren des ausgewählten Darlehenskalkulators nicht zu besserem Verständnis in Bezug auf die darin enthaltenen Konzepte und Zusammenhänge führt. Zudem fördert die Webseite zu wenig relevante kognitive Aktivitäten, wie das Evaluieren von Informationen 
und Bedeutungen sowie das Ableiten von Konsequenzen; es entstehen oder es manifestieren sich einige Fehlkonzepte, die Entscheidungen beeinflussen können. Online verfügbare Informationen zu Finanzprodukten tragen damit nicht zwangsläufig zu einer Förderung von Finanzkompetenz bei.

Um informelles Lernen aus Online-Informationen zu Finanzprodukten wirksam zu gestalten, sollten diese Informationen und deren Darbietung Denkprozesse höherer Ordnung stimulieren (Mayer 2009; Damnik et al. 2013). Individuen sollten dabei unterstützt werden, neue Informationen zu selektieren, $\mathrm{zu}$ organisieren und in bestehende Wissensstrukturen zu integrieren. Dazu sollten die relevanten Konzepte und Zusammenhänge (z. B. zur Annuitätenrechnung) präsentiert und auf weniger relevante Informationen verzichtet werden. Weitere Ansatzpunkte für die Gestaltung der Webseiten und die lernförderliche Präsentation von Informationen liefern die Prinzipien multimedialen Lernens (Mayer 2017), die in Folgestudien umgesetzt und auf ihre Wirkung hin überprüft werden sollten.

\section{Diskussion}

Die Ausführungen zur Messung von Finanzkompetenz von Förster und Kraitzek haben die Bedeutung von Messverfahren, die nicht nur Single- oder Multiple-Choice-Items verwenden und nicht ausschließlich auf Faktenwissen zielen, verdeutlicht. Weiterhin haben die beiden Studien von Wuttke, Siegfried und Aprea sowie Hommel und Fürstenau gezeigt, dass der Einsatz von komplexen Verfahren zur Messung von Finanzkompetenz einen Mehrwert gegenüber den genannten bestehenden Verfahren hat. Konkret lässt sich benennen, dass die Studien nicht nur Faktenwissen, sondern auch Entscheidungsverhalten berücksichtigen. Hinzu kommt, dass sie jeweils einen weiteren Schwerpunkt berücksichtigen. Dieser betrifft in der Studie von Wuttke, Siegfried und Aprea den Gender Gap, in der Studie von Hommel und Fürstenau das informelle Lernen sowie darauf bezogene kognitive Prozesse und Fehlkonzepte. Einzelne Aspekte der Beiträge sollen im Folgenden noch etwas näher betrachtet werden.

Aus dem Beitrag von Förster und Kraitzek geht hervor, dass die meisten Verfahren im internationalen und deutschen Kontext den Fokus auf kognitive Dispositionen im Assessment finanzieller Kompetenz legen. Deutlich wird jedoch auch, dass in neueren Entwicklungen eine ausgeprägtere Kompetenzorientierung zu finden ist, die sich durch einen stärkeren Situations- und lebensweltlichen Bezug der Aufgaben sowie komplexere Fragestellungen auszeichnen. Dabei kann diskutiert werden, wie komplex die Situation und der Problemlöseprozess für die Probanden in neuen Assessmentverfahren ausgestaltet werden sollten, um möglichst authentische Entscheidungsprozesse zu simulie- 
ren. Hier sollte die Komplexität der Aufgaben und der Situationsbeschreibungen weiter erhöht werden, um eine höhere ökologische Validität zu erzielen und der Realität noch besser gerecht zu werden. Förster und Kraitzek validieren hier in einem nächsten Schritt gerade die Lösung komplexer, alltagsnaher Problemszenarien im Finanzkontext, die aus mehreren Seiten realer Informationen (z. B. Aktienkurse, Anlagebedingungen verschiedener Produkte, etc.) bestehen. Schüler*innen müssen hier u. a. wichtige von unwichtigen Informationen abgrenzen und ihre Entscheidung fachlich begründen.

Die Studie von Wuttke, Siegfried und Aprea hat gezeigt, dass es zwei Testbereiche gibt, in denen weibliche Probanden tendenziell oder signifikant besser abschneiden als männliche Probanden. Es gibt jedoch wenige Studien, die auch einen Leistungsvorsprung weiblicher Teilnehmer bestätigen (z. B. Hill \& Asarta 2016; Jang et al. 2014; Walstad et al. 2010). Die wohl bekannteste ist hier die aktuelle PISA-Studie, gemäß der Schülerinnen in Ländern, wie Spanien, Polen oder Litauen, signifikant bessere Leistungen im Financial Literacy Tool erzielen als Schüler (OECD 2017a). Dennoch stellt sich das Phänomen des Gender Gaps in einzelnen Studien ganz unterschiedlich dar, und es wird eine Reihe an Hintergrundinformationen herangezogen, um den Gender Gap zu klären. Dazu gehören Unterschiede in individuellen Merkmalen zwischen Männern und Frauen, Unterschiede in sozio-ökonomischen Merkmalen, aber auch länderspezifische Merkmale oder Unterschiede in Lerngelegenheiten, die Männer und Frauen nutzen, um etwas über Finanzen in Erfahrung zu bringen (Bucher-Koenen et al. 2017). Hier stellt sich die Frage, ob noch weitere Hintergrundvariablen in die Studie mit einbezogen werden können, um nähere Informationen über den Vorteil für weibliche Probanden zu erhalten. Weiterhin wird das Aufgabenformat als Erklärungsfaktor für den Gender Gap herangezogen, da Ergebnisse zeigen, dass vor allem männliche Lernende bei Multipleoder Single-Choice-Items bevorteilt werden. Im Gegenzug stellt sich die Frage, ob es gegebenenfalls gerade die SJT sind, die Vorteile für weibliche Probanden mit sich bringen oder ob weitere Hintergrundvariablen zu berücksichtigen sind, um den Vorteil für diese Gruppe zu erklären. Um diese Frage näher zu beleuchten, ist der zentrale Prädiktor das Informations- und Kaufverhalten bzw. die Fähigkeit, Bedürfnisse aufzuschieben, näher zu untersuchen. Es ist zu klären, ob es deskriptive Analysen zu der eingesetzten Skala gibt, die Unterschiede zwischen Männern und Frauen zeigen und insofern den Gender Gap erklären könnten. Gleichzeitig wird deutlich, dass die Berücksichtigung des Prädiktors Informations- und Kaufverhalten bzw. Bedürfnisaufschub in den Regressionsanalysen eine Auswirkung auf den Gender Gap hat. Dies kann Anlass sein, in Folgestudien weitere Faktoren aufzunehmen, die mit dem Kaufverhalten und Bedürfnisaufschub in Verbindung stehen. Vorstellbar sind in diesem Zusammenhang Skalen, die Einstellung zu Geld, zu nachhaltigem Konsum oder zum reflektierten Umgang mit Werbung messen. Dabei könnten sich Unterschiede zwischen Männern und Frauen zeigen, die weiter zur Erklärung 
des Gender Gaps dienen. Das regressionsanalytische Vorgehen von Wuttke, Siegfried und Aprea ließe sich folglich um weitere Hintergrundvariablen erweitern, zusätzlich könnte die Berücksichtigung von Interaktionseffekten eine noch differenziertere Analyse des Gender Gaps ermöglichen. Auch eine Erweiterung der Stichprobe bspw. um andere Alterskohorten ist denkbar.

Die Studie von Hommel und Fürstenau hat gezeigt, dass die Exploration von Internetseiten das informelle Lernen und die Kompetenzentwicklung kaum unterstützen. Dadurch wird die Bedeutung, Finanzkompetenz in formalen Settings zu fördern oder informelles und formales Lernen zu koppeln, unterstrichen. Es ist jedoch zu berücksichtigen, dass das Ergebnis informellen Lernens unterschiedliche Ursachen haben kann: Einerseits sind die Webseiten zwar zur Informationsbeschaffung gedacht, aber Banken verfolgen vermutlich nicht in erster Linie die Intention, umfassende Lernprozesse anzustoßen. Möglicherweise unterscheiden sich diesbezüglich jedoch Direktbanken von traditionellen Banken. Hinzu kommt, dass die Webseiten auf den ersten Blick wenig kognitiv aktivierend wirken. Vielmehr wird eine Informationsfülle dargeboten, die im Zweifel sogar zur Überforderung der Nutzer*innen führen kann. Dies gilt insbesondere dann, wenn Anwender*innen über mangelnde fachliche oder digitale Kompetenzen verfügen. $\mathrm{Zu}$ überlegen wäre für weitere ähnlich gelagerte Studien, eine Skala zu digitalen Kompetenzen einzusetzen oder den Umgang mit den Webseiten, z. B. über Log-Files, mit zu erfassen. Auch eine Erweiterung der Stichprobe über Studierende hinaus wäre denkbar, um zu analysieren, ob sich vergleichbare Ergebnisse auch für andere Personengruppen zeigen. Möglich wäre beispielsweise ein Zusammenhang zwischen informellen Lernprozessen und der Intention, Eigentum zu erwerben. $\mathrm{Zu}$ berücksichtigen ist jedoch auch, dass die Webseiten die persönliche Beratung nicht ersetzen, sondern die Kunden gerade zu einem persönlichen Gespräch mit einem Berater oder einer Beraterin motivieren sollen. Eine Stärke der Studie kann in dem komplexen Messverfahren und dem umfangreichen Datenpool gesehen werden. Insbesondere die Daten zu den Fehlkonzepten liefern zentrale Implikationen für formale Lernprozesse. Einerseits zeigen die Ergebnisse, dass es Konzepte, wie Zins, Tilgung oder Zinsfestschreibung, sind, die Studierende nicht richtig verstehen. Diese Themen könnten von der Fachwissenschaft und der Fachdidaktik noch einmal aufgegriffen werden, um ein Conceptual Understanding oder ein Conceptual Change bei Studierenden anstoßen zu können.

Insgesamt lässt sich feststellen, dass im Symposium der Stand zum Assessment aufgearbeitet wurde und die vorgestellten Studien Hinweise darauf geben, wie die Messung von Finanzkompetenz verbessert und die Forschung weiterentwickelt werden kann, sei es zum Gender Gap, sei es zum informellen Lernen oder zu Fehlkonzepten. 


\section{Literatur}

Aprea, C., Wuttke, E., Leumann, S. \& Heumann, M. (2015). Kompetenzfacetten von Financial Literacy: Sichtweisen verschiedener Akteure. In J. Seifried, S. Seeber \& B. Ziegler (Hrsg.), Jahrbuch der berufs- und wirtschaftspädagogischen Forschung. Schriftenreihe der Sektion Berufs- und Wirtschaftspädagogik der Deutschen Gesellschaft für Erziehungswissenschaft (DGfE) (S. 11-22). Opladen: Barbara Budrich.

Aprea, C. \& Wuttke, E. (2016). Financial literacy of adolescent and young adults: setting the course for a competence-oriented assessment approach. In C. Aprea, E. Wuttke, K. Breuer, N.K. Keng, P. Davies, B. GreimelFuhrmann \& J. Lopus (eds.), International Handbook of Financial Literacy (pp. 397-414). Singapore: Springer.

Aprea, C., Wuttke, E., Breuer, K., Keng, N.K., Davies, P., Greimel-Fuhrmann, B. \& Lopus, J. (2016). Financial literacy in the twenty-first century: An introduction to the International Handbook of Financial Literacy. In C. Aprea, E. Wuttke, K. Breuer, N.K. Keng, P. Davies, B. Greimel-Fuhrmann \& J. Lopus (eds.), International Handbook of Financial Literacy (pp. 14). Singapore: Springer.

Barry, D. (2014). Die Einstellung zu Geld bei jungen Erwachsenen. Eine Grundlegung aus wirtschaftspädagogischer Sicht. Wiesbaden: Springer VS.

Bender, N. \& Barry, D. (2013). Adaption of the TRSQ for Financial Behavior. In K. Beck \& O. Zlatkin-Troitschanskaia (eds.), From Diagnostics to Learning Success. Proceedings in Vocational Education and Training (pp. 213-224). Rotterdam: Sense.

Breuer, K. (1999). Befähigung zur Selbstregulation in der beruflichen Aus- und Weiterbildung (Fragebogen zu Lernen und Denken). Deutsche Fassung des Trait Self-Regulation Questionnaire (TSRQ) nach O'Neil \& Herl. Unveröffentlichter Fragebogen. Mainz.

Bucher-Koenen, T., Lusardi, A., Alessie, R., van Rooij, M. (2017). How Financially Literate Are Women? An Overview and New Insights. Journal of Consumer Affairs, 51(2), 255-238.

Cerasoli C. P., Alliger G. M., Donsbach, J. S., Mathieu, J. E., Tannenbaum, S. I. \& Orvis, K. A. (2018) Antecedents and Outcomes of Informal Learning Behaviors: A Meta-Analysis. Journal of Business Psychology, 33, 203230

Chi, M. T. H., Bassok, M., Lewis, M. W., Reimann, P. \& Glaser, R. (1989). Self-Explanations: How Students Study and Use Examples in Learning to Solve Problems. Cognitive Science, 13, 145-182.

Council for Economic Education CEE (2013). National Standards for Financial Literacy. New York.

Damnik, G., Proske, A., Narciss, S. \& Körndle, H. (2013). Informal Learning with Technology: The Effects of Self-Constructing Externalizations. The Journal of Educational Research, 106, 431-440. 
Dennen, V. P. \& Wang, M. (2002). The Keyboard-Based Job Coach: Informal Learning via the Internet. Advances in Developing Human Resources, 4(4), 440-450.

Eitel, A., Dersch, A.-S. \& Renkl, A. (2019). Wissenschaftliche Annahmen und Fehlkonzepte angehender Lehrkräfte über das Lernen mit Multimedia. Unterrichtswissenschaft, 47, 451-474.

Förster, M., Happ, R. \& Molerov, D. (2017). Using the U.S. Test of Financial Literacy in Germany - Adaption and Validation. Journal of Economic Education, 48(2), 123-135.

Fürstenau, B., Hommel, M., Leopold, C., Ponce, H. \& López, M. (2016). Analysis of Banks' Online Information Regarding Mortgages as a Basis for Financially Literate Decision-Making in First-Time Homebuying. In E. Wuttke, J. Seifried \& S. Schumann (eds.), Economic Competence and Financial Literacy of Young Adults. Status and Challenges (pp. 63-79). Opladen u. a.: Barbara Budrich.

Fürstenau, B. \& Hommel, M. (2018). Contribution of bank webpages to the development of young adults' financial competence about mortgages. Empirische Pädagogik, 32(3/4), 434-459.

Fürstenau, B. \& Hommel, M. (2019). Developing financial competence about mortgage loans by informal learning using banks' online calculators. Empirical Research in Vocational Education and Training, 11(10), 1-33.

Geiger, J.-M., Meretz, U. \& Liening, A. (2016). Systematisierung deutschsprachiger Studien zur Kompetenzerfassung von financial literacy. Zeitschrift für ökonomische Bildung, 5, 72-93.

Greimel-Fuhrmann, B. \& Silgoner, M. (2018). Analyzing the Gender Gap in Financial Literacy. International Journal for Infonomics (IJI), 11(3), $1779-1787$.

Grohs-Müller, S. (2018). Schüler/innen und ihr Umgang mit Geld. Dissertation, Wirtschaftsuniversität Wien.

Hausmann, V. \& Retzmann, T. (2014). US-amerikanische Tests für die Ökonomische Bildung - Ein Modell für Deutschland? In T. Retzmann (Hrsg.), Ökonomische Allgemeinbildung in der Sekundarstufe I und Primarstufe (S. 330-345). Schwalbach/Ts.: Wochenschau Wissenschaft.

Happ, R. \& Förster, M. (2019). The relationship between migration background and knowledge and understanding of personal finance of young adults in Germany. International Review of Economics Education, 30(1), $1-14$.

Hill, A. T. \& Asarta, C. J. (2016). Gender and student achievement in personal finance: Evidence from keys to financial success. In C. Aprea, E. Wuttke, K. Breuer, N.K. Keng, P. Davies, B. Greimel-Fuhrmann \& J. Lopus (eds.), International Handbook of Financial Literacy (pp. 545-567). Singapore: Springer.

Holland, A. A. (2019). Effective principles of informal online learning design: A theory-building metasynthesis of qualitative research. Computers $\&$ Education, 128, 214-226. 
Hommel, M., Fürstenau, B., Leopold, C., Ponce, H. \& López, M. (2017). Beitrag von Banken-Webseiten zur Entwicklung der Finanzkompetenz potentieller Darlehensnehmer/innen über Baufinanzierungen. In J. Seifried, S. Seeber \& B. Ziegler (Hrsg.), Jahrbuch der berufs- und wirtschaftspädagogischen Forschung 2017. Schriftenreihe der Sektion Berufs- und Wirtschaftspädagogik der Deutschen Gesellschaft für Erziehungswissenschaft (DGfE) (S. 97-111). Opladen: Barbara Budrich.

Jang, K., Hah, J. \& Park, H. (2014). Comparison of financial literacy between Korean and U.S. High School Students. International Review of Economics Education, 16, 22-38.

Jerusalem, M. \& Schwarzer, R. (1999). Allgemeine Selbstwirksamkeit. In R. Schwarzer \& M. Jerusalem (Hrsg.), Skalen zur Erfassung von Lehrer- und Schülermerkmalen (S. 13-14) Berlin: Freie Universität.

Kahmann, J. (2014). Entwicklung und Validierung eines Situational Judgement Tests (SJT) zur Erfassung sozialer Kompetenzen von Studienplatzbewerbern und -interessenten der Human und Zahnmedizin. Dissertation. Heidelberg.

Kraitzek, A. (in Vorbereitung). Utilization of the U.S. Test of Financial Knowledge in Germany - Adaption and Validation. Annual Meeting of the American Educational Research Association (AERA), San Francisco.

Krippendorf, K. (2012). Content Analysis: An Introduction To Its Methodology. London: Sage.

Lindner, M. A., Strobel, B. \& Köller, O. (2015). Multiple-Choice-Prüfungen an Hochschulen? Ein Literaturüberblick und Plädoyer für mehr praxisorientierte Forschung. Zeitschrift für Pädagogische Psychologie, 29(3-4), $133-149$.

Lusardi, A. \& Mitchell, O. S. (2009). How Ordinary Consumers Make Complex Economic Decisions: Financial Literacy and Retirement Readiness. NBER Working Paper, No. 15350, Online: https://www.nber.org/papers/w15350.pdf (03.12.2019).

Lusardi, A. \& Mitchell, O. S. (2011). Financial Literacy around the world: an overview. Journal of Pension Economics and Finance, 10(4), 497-508.

Lusardi, A. \& Mitchell, O. S. (2014). The Economic Importance of Financial Literacy: Theory and Evidence. Journal of Economic Literature, 52(1), 544.

Maier, M., Rothmund, T., Retzbach, A., Otto, L \& Besley, J. C. (2014) Informal Learning Through Science Media Usage. Educational Psychologist, 49(2), 86-103.

Mayer, R. E. (2009). Multimedia learning ( $2^{\text {nd }}$ ed.). New York: Cambridge University Press.

Mayer, R. E. (2017). Using multimedia for e-learning. Journal of Computer Assisted Learning, 33, 403-423.

Mayring, P. (2015). Qualitative Inhaltsanalyse: Grundlagen und Techniken (12. Aufl.). Weinheim: Beltz.

McDaniel, M. A., Hartmann, N. S., Whetzel, D. L. \& Grubb, W. L. (2007). Situational judgment tests, response instructions, and validity: A metaanalysis. Personnel Psychology, 60, 63-91. 
OECD (2013). PISA 2012 Assessment and Analytical Framework: Mathematics, Reading, Science, Problem Solving and Financial Literacy, OECD Publishing. Online: http://dx.doi.org/10.1787/9789264190511-en (28.11.2019).

OECD (2017a). PISA 2015 Results. Students 'Financial Literacy. (Volume VI). Paris: OECD Publishing.

OECD (2017b). G20/OECD INFE report on adult financial literacy in G20 countries. Online: http://www.oecd.org/daf/fin/financial-education/G20OECD-INFE-report-adult-financial-literacy-in-G20-countries.pdf (28.11.2019).

Prenzel, M., Kristen, A., Dengler, P., Ettle, R. \& Beer, T. (1996). Selbstbestimmt motiviertes und interessiertes Lernen in der kaufmännischen Erstausbildung. In K. Beck \& H. Heid (Hrsg.), Lehr-Lern-Prozesse in der kaufmännischen Erstausbildung. Wissenserwerb, Motivationsgeschehen und Handlungskompetenzen (S. 108-127). Stuttgart: Franz Steiner.

Ray, J. \& Najman, J. (1986). The Generaliziability of Deferment of Gratification. Journal of Social Psychology, 126, 117-119.

Reardon, S. F., Kalogrides, D., Fahle, E. M., Podolsky, A., \& Zárate, R. C. (2018). The Relationship between Test Item Format and Gender Achievement Gaps on Math and ELA Tests in 4th and 8th Grade. Educational Researcher, 47(5), 284-294.

Rosendorfer, T. (2000). Kinder und Geld. Gelderziehung in der Familie. Frankfurt a. M.: Campus.

Rudeloff, M. (2019). Der Einfluss informeller Lerngelegenheiten auf die Finanzkompetenz von Lernenden am Ende der Sekundarstufe I. Wiesbaden: Springer.

Saygin, P. O. (2019). Gender bias in standardized tests: evidence from a centralized college admissions system. Empirical Economics. https://doi.org/ 10.1007/s00181-019-01662-z.

Schürkmann, S. (2017). FILS: Financial Literacy Study. Validierung und Analyse einer schülerorientierten Financial Literacy. Oldenbourg: DeGruyter.

Schürkmann, S. \& Schuhen, M. (2013). Kompetenzmessung im Bereich financial literacy. Ergebnisse zum Umgang mit Online-Rechnern aus der FILSStudie. Zeitschrift für ökonomische Bildung, 1, 73-89.

Siegfried, C. \& Wuttke, E. (2019). Are Multiple Choice Items unfair? And if so, for whom? Citizenship, Social and Economics Education, 18(3), 123127.

Temme, D. \& Hildebrandt, L. (2008). Gruppenvergleiche bei hypothetischen Konstrukten - Die Prüfung der Ubereinstimmung von Messmodellen mit der Strukturgleichungsmethodik. Schriftenreihe Economic Risk SFB 649 Papers, Discussion Paper 2008-042.

Walstad, W. B, Rebeck, K. \& MacDonald, R. A. (2010). The effects of financial education on the financial knowledge of High School students. The Journal of Consumer Affairs, 44(2), 336-357.

Walstad, W. B. \& Rebeck, K. (2016a). Basic Finance Test: Examiner's Manual. New York: Council for Economic Education.

Walstad, W. B. \& Rebeck, K. (2016b). Test of Financial Knowledge: Examiner's Manual. New York: Council for Economic Education. 
Walstad, W. B. \& Rebeck, K. (2016c). Test of Financial Literacy: Examiner 's Manual. New York: Council for Economic Education.

Weinert, F. E. (2001). Concept of Competence: A Conceptual Clarification. In D. S. Rychen \& L. H. Salganik (Hrsg.), Defining and Selecting Key Competencies (pp. 45-65). Seattle, Toronto: Hogrefe and Huber.

Weinert, F. E. (2002). Vergleichende Leistungsmessung in Schulen - eine umstrittene Selbstverständlichkeit. In F. E. Weinert (Hrsg.), Leistungsmessungen in Schulen (S. 17-31). Weinheim: Beltz.

Werquin, P. (2010). Recognising Non-Formal and Informal Learning. Outcomes, Policies and Practices. OECD Publishing, Paris.

Winther, E. (2006). Motivation in Lernprozessen. Konzepte in der Unterrichtspraxis von Wirtschaftsgymnasien. Wiesbaden: DUV.

Wuttke, E. \& Aprea, C. (2018). A situational judgement approach for measuring young adults' financial literacy. Empirische Pädagogik, 32, 272-292.

Wuttke, E., Siegfried, C., \& Aprea, C. (in Vorbereitung). Measuring financial literacy with a Situational Judgement Test - do some groups really perform worse or is it the measuring instrument? 
Appendix: Übersicht über Messinstrumente zur Financial Literacy

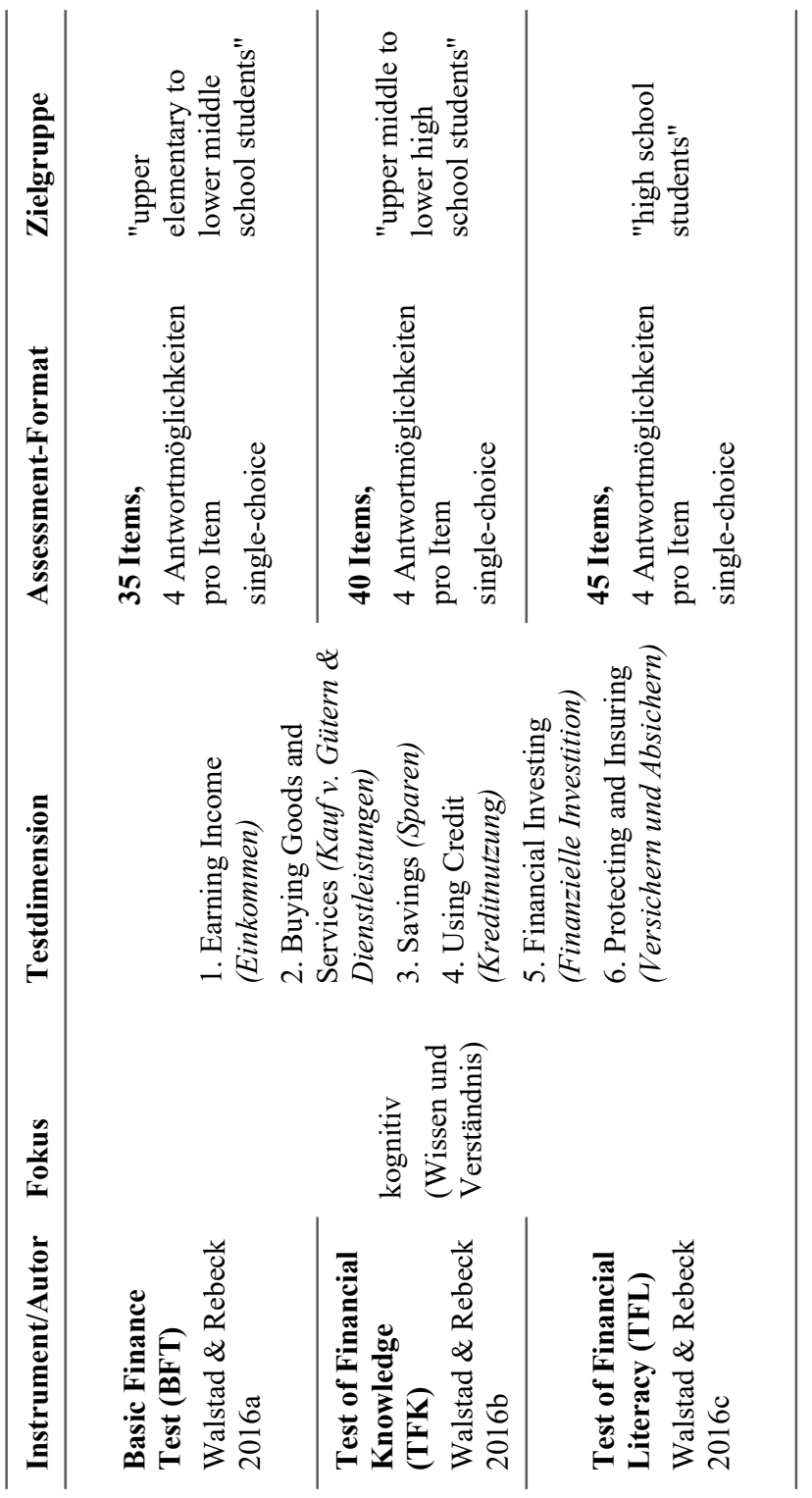




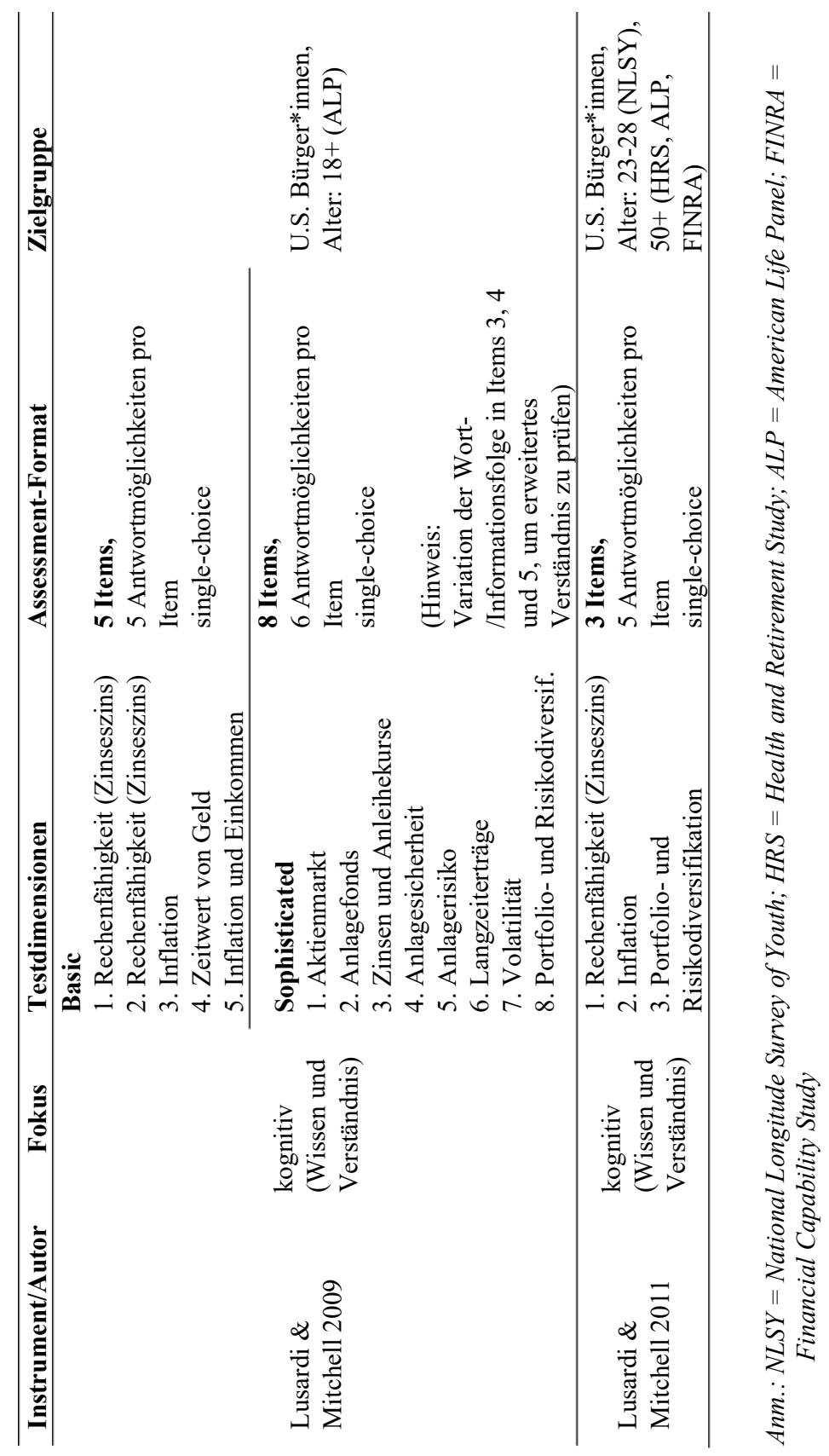




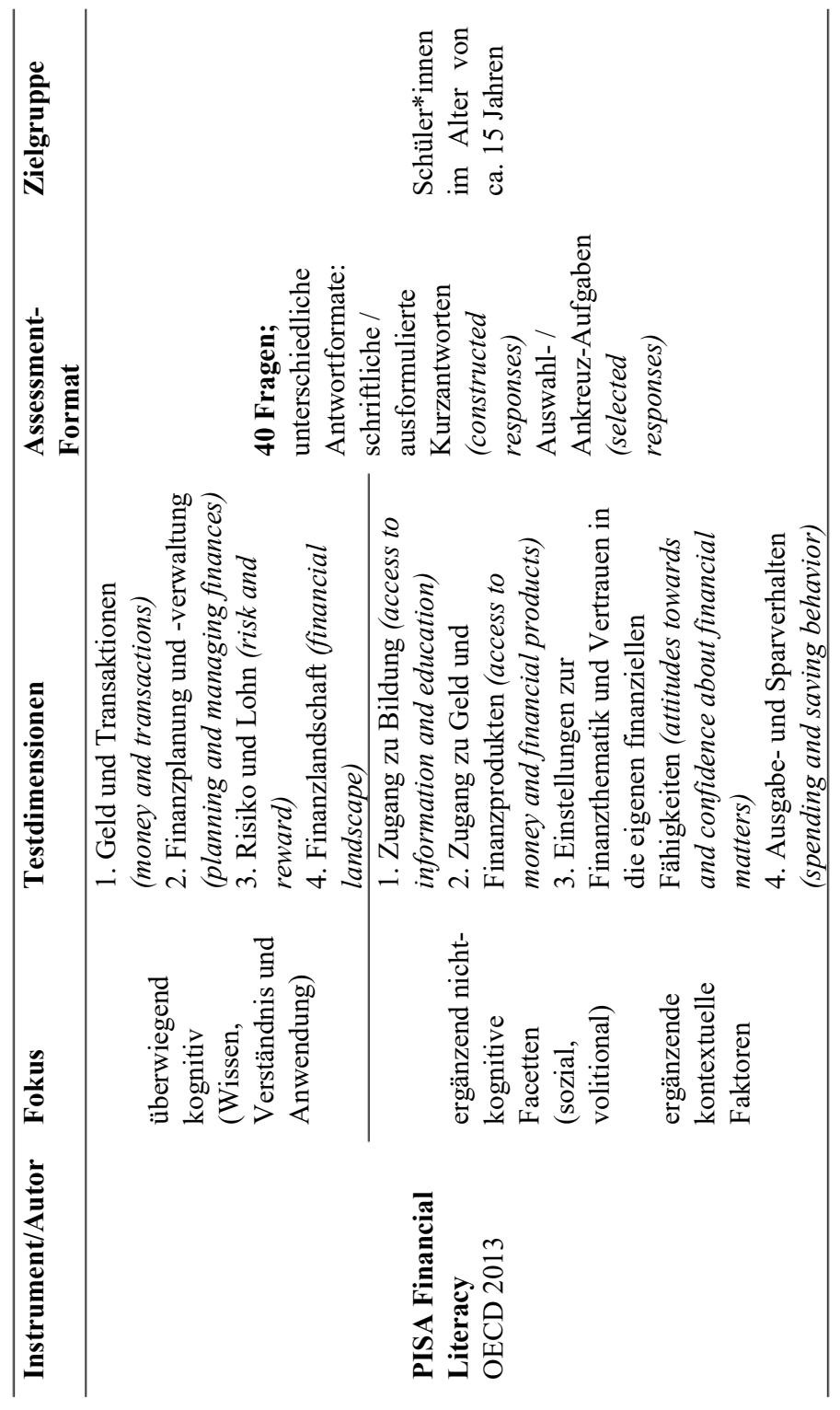




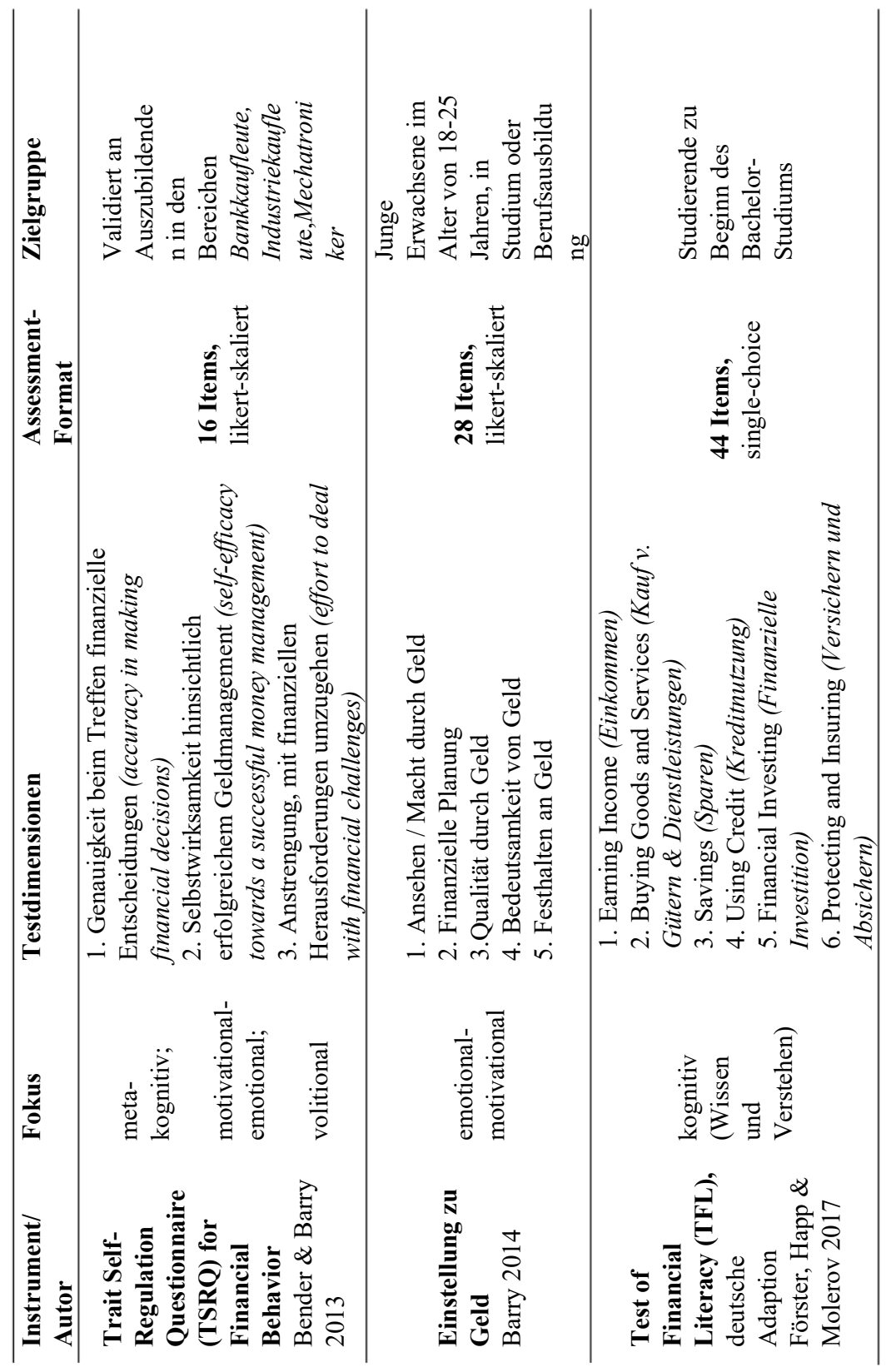




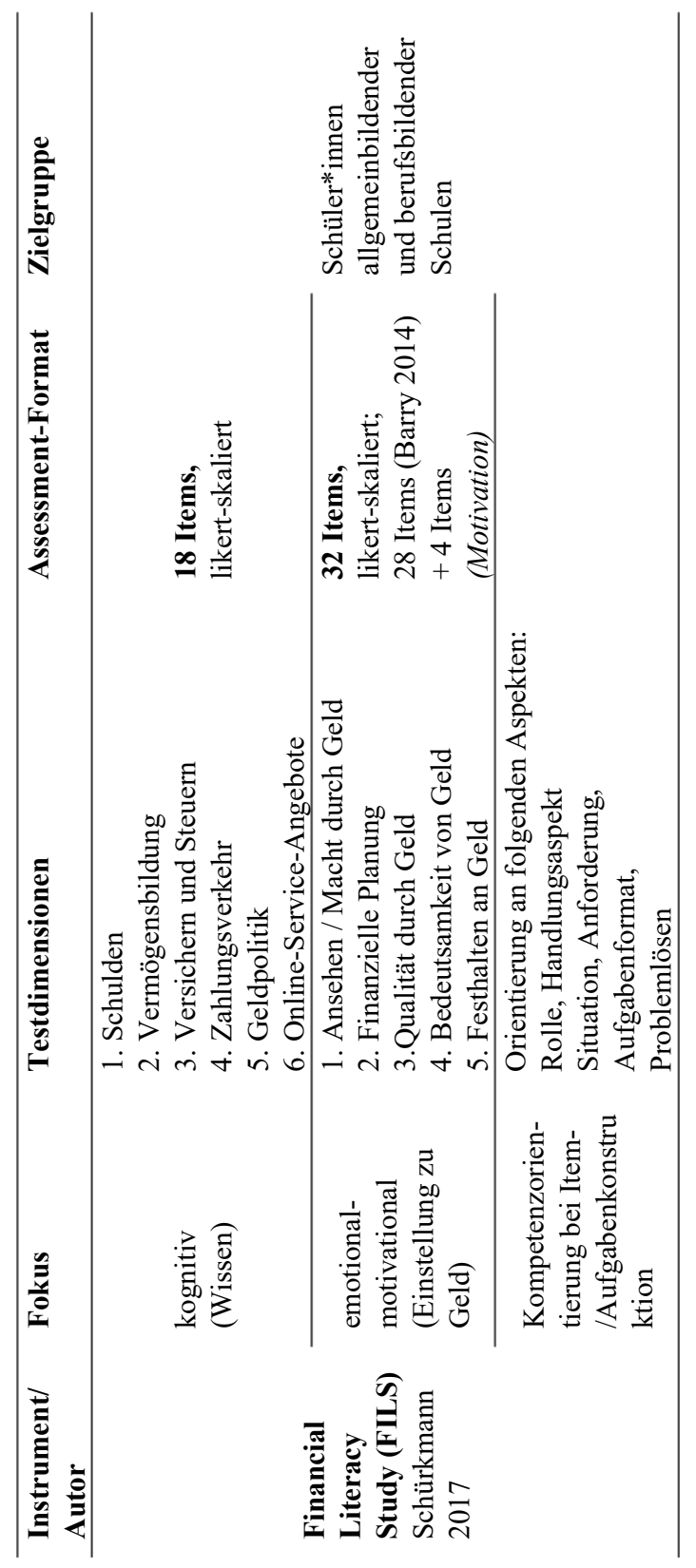




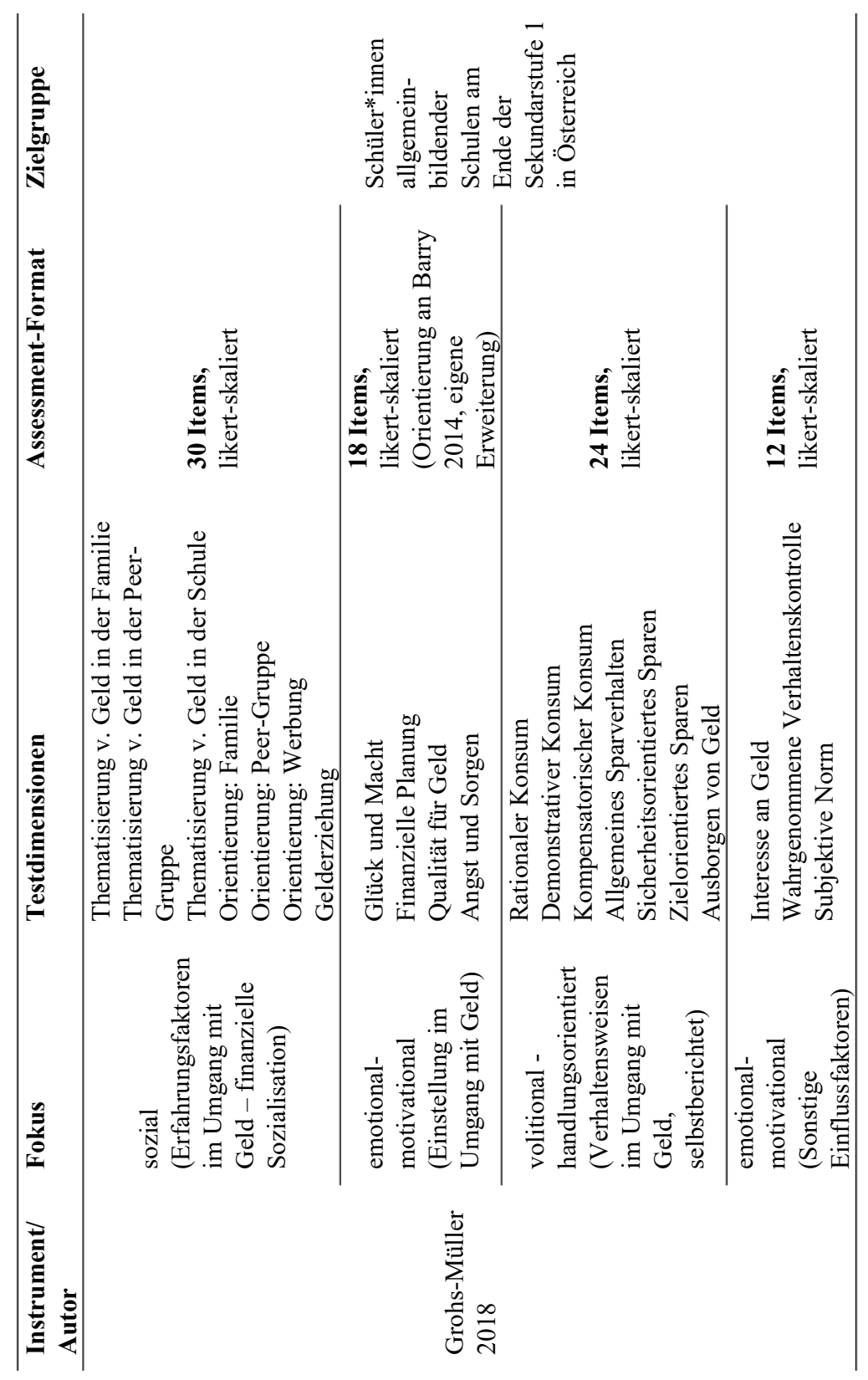




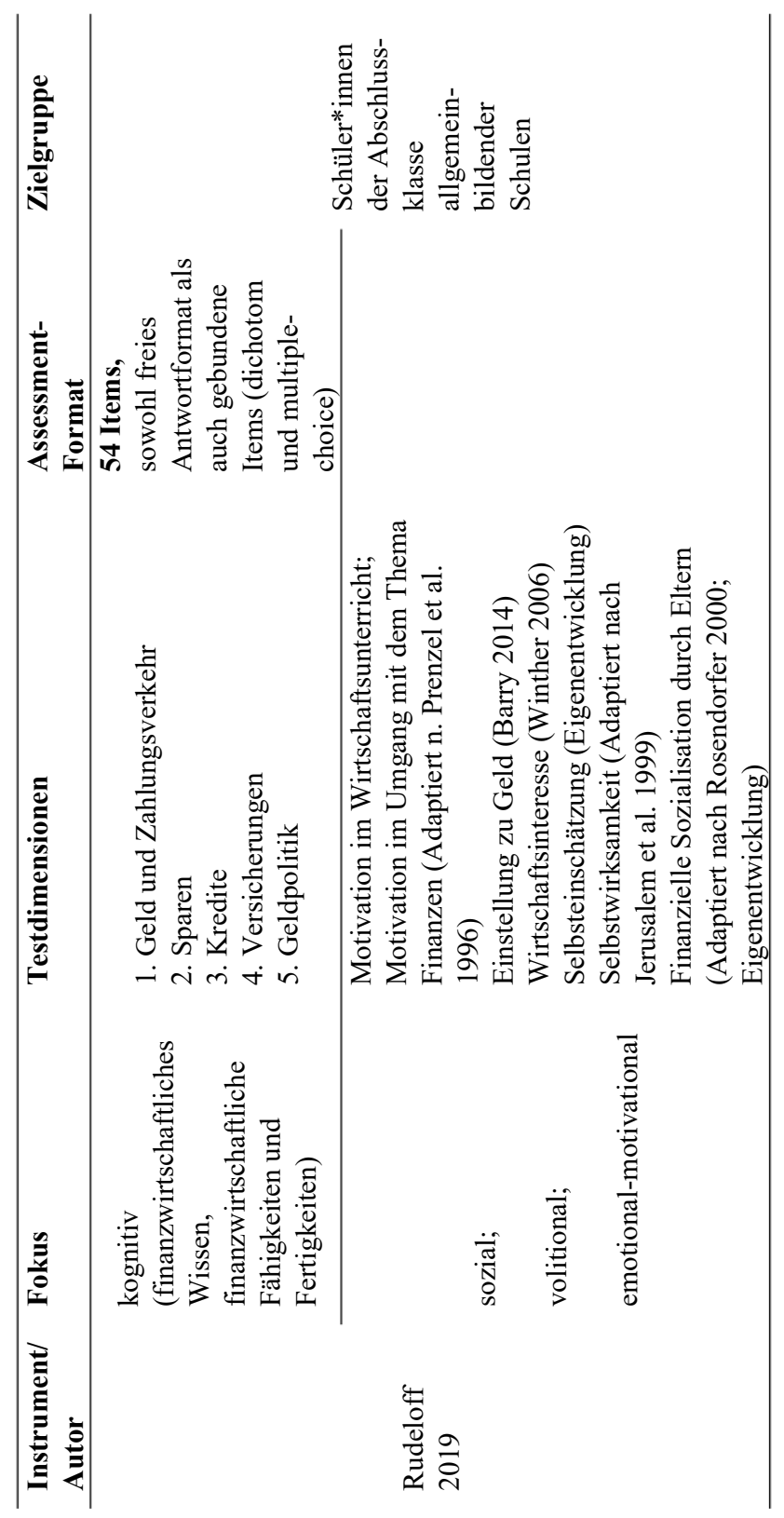




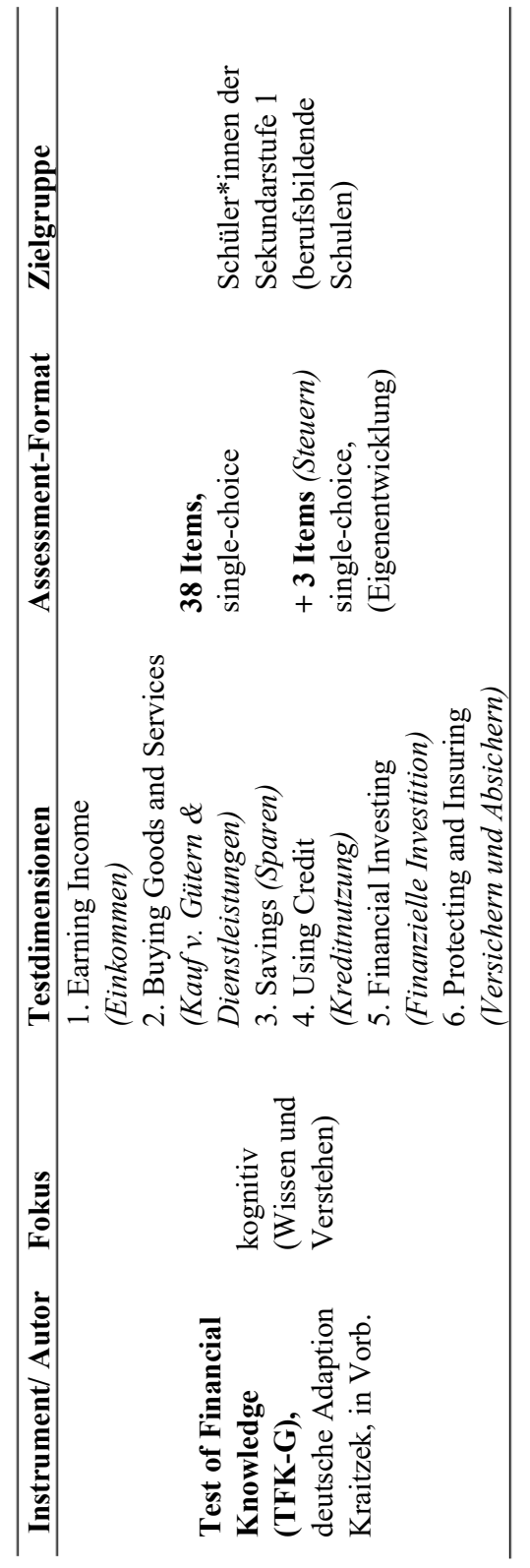




\title{
Theoretische Reflexionen zur Didaktik der Berufsbildung für nachhaltige Entwicklung (BBNE) aus der Perspektive der Modellversuchsforschung
}

\author{
Andreas Fischer, Marc Casper, Karina Kiepe, Harald Hantke, Jan Pranger
} und Sören Schütt-Sayed

\section{Einleitung}

Das Jahr 2019 war geprägt vom Themenkomplex „Nachhaltigkeit“. Die Medienwirksamkeit der „Fridays for Future“-Bewegung ist nur eines von vielen Beispielen für die Relevanz dieses, oft emotional aufgeladenen, gesamtgesellschaftlichen Diskurses für das Bildungssystem. Unter den jugendlichen Demonstrant*innen befinden sich letztendlich auch die Berufsschüler*innen von heute und morgen.

Junge Menschen fordern Reflexion und Transformation im Sinne der regulativen Idee der nachhaltigen Entwicklung, eine Transformation, die wirtschaftliche Ziele und die konkrete Ausgestaltung von Berufsarbeit fundamental betrifft. Diese Idee einer nachhaltigen Entwicklung, die insbesondere im UNESCO-Weltaktionsprogramm ausformuliert ist, ist jedoch anspruchsvoll und wertebasiert, damit erklärungs- und aushandlungsbedürftig. Sie fordert Zukunftsorientierung und Verantwortung auf unterschiedlichen Ebenen in oft abstrakten, eher kursorischen Ansprüchen. Im Sinne der Brundtland-Definition ist ,nachhaltige Entwicklung eine Entwicklung, die den Bedürfnissen der heutigen Generation entspricht, ohne die Möglichkeiten künftiger Generationen $\mathrm{zu}$ gefährden, ihre eigenen Bedürfnisse $\mathrm{zu}$ befriedigen.“ (WCED 1987, Ch. 1 § 49). Die Spannweite dieser Vision geht offensichtlich weit über das in 2019 so prominente Thema „Klimawandel“ hinaus, wie es auch die Vielfalt der 17 Nachhaltigkeitsziele der UN auf einer weiteren Konkretisierungsebene vermittelt. Doch wie lässt sich ein solch umfassender Anspruch für Berufsbildung konkretisieren? Wie lässt er sich in konkretes, domänenspezifisches Berufshandeln und in Lernprozesse übersetzen, auch angesichts betrieblicher Realitäten und vorherrschender Sachzwänge? Welche rationalen Kriterien pädagogischer Professionalität müssen angesetzt werden, um konstruktiv zu wirken und nicht im Normativen verhaftet zu bleiben?

Die UN-Dekade „Bildung für nachhaltige Entwicklung“ widmet sich diesen Fragen unter dem Motto „Vom Projekt zur Struktur“ (Hemkes 2014). Es gilt, die Erfahrungen aus diversen Modellversuchen und Pilotprojekten derart $\mathrm{zu}$ bündeln, zu reflektieren, zu verallgemeinern und zu verstetigen, dass sich 
nachhaltige Entwicklung in der Breite der Berufsbildung als Zieldimension etablieren kann. Hierzu förderte das Bundesinstitut für Berufsbildung (BIBB) - als eine von mehreren Förderlinien - den Schwerpunkt „Entwicklung von domänenspezifischen Nachhaltigkeitskompetenzen in kaufmännischen Berufen" (vgl. www.bbne.de).

In sechs Modellversuchen wurden diverse Ergebnisse erzielt: von Instrumenten für den Einsatz in der betrieblichen Ausbildung, über umfassende Fortbildungskonzepte bis zu komplexen e-Learning-Angeboten. Gemeinsam war allen Modellversuchen ein didaktisches Gestaltungsinteresse: Im Spannungsfeld betrieblicher Routinen und transformativer Ansprüche mussten neue Formen des Lernens und Mitgestaltens erprobt werden. Diese Ergebnisse sind über die zentrale Webseite des BIBBs (www.bbne.de) zugänglich. Zusätzlich entstanden zahlreiche Berichte, Praxishandreichungen und ein abschließender Sammelband, in dem auch die programmübergreifenden Ergebnisse zur Kompetenzstruktur und zu didaktischen Kriterien dargestellt werden (Schütt-Sayed, Casper \& Vollmer, im Erscheinen).

Über jene pragmatisch orientierten Ergebnisse hinaus verfolgt dieser Jahrbuchbeitrag das noch offene Anliegen einer theoretischen Gesamtreflexion. Das zweite Kapitel widmet sich zunächst der Frage, welche fachwissenschaftliche Fundierung eine BBNE in kaufmännischen Berufen des Handels braucht, damit fachdidaktische Überlegungen nicht gegenstandslos, um nicht zu sagen zahnlos, bleiben. Schließlich ist „Lernen stets eine kognitiv-emotional-soziale Bezugnahme auf etwas - als Gegenstand des Lernens. Eine Untersuchung dieses Lernens muss auch die Eigen-Strukturen dieses »Etwas« in Rechnung stellen, um beurteilen zu können, inwiefern das Lernen seinem Gegenstand gerecht wird oder diesen verfehlt, und um aufzuklären, inwiefern Sinn und Zweck des Lernens mit Sinn und Zweck des Lerngegenstandes korrespondieren." (Rhein 2015, 350). Anhand des Modellversuchs GeKoNaWi ${ }^{1}$ wird der Implikationszusammenhang fachdidaktischer Bezüge und methodischer Entscheidungen einer BBNE exemplarisch illustriert. Im dritten Kapitel wird hieran anschließend das Potenzial innovativer und transformationsorientierter Methoden für eine subjektorientierte BBNE reflektiert. Am Beispiel der systemischen Visualisierung aus dem Modellversuch Pro-DEENLA wird diskutiert, inwieweit Perspektivwechsel, Widerspruchsbewusstsein und transformatorische Aushandlungsprozesse in kaufmännischen Kontexten methodisch ermöglicht werden können. Das vierte Kapitel bildet ein vorläufiges Fazit mit Ausblick. Es führt dazu mit dem Begriff des „Lebendigen Lernens“ im Sinne der humanistischen Psychologie analytisch zusammen, welche Prinzipien und produktiven Spannungsfelder die Didaktik der BBNE aus der Perspektive der Modellversuchsforschung kennzeichnen.

1 Geschäftsmodell- und Kompetenzentwicklung für nachhaltiges Wirtschaften im Handel (GeKoNaWi) 


\section{Die kaufmännische Berufsbildung für nachhaltige Entwicklung und ihre fachwissenschaftliche Fundierung - am Beispiel des Modellversuchs GEKONAWI}

Die BBNE ist bis heute weder in der Praxis noch in der Forschung hinreichend etabliert. Ein wesentlicher Grund dafür ist, dass die BBNE bislang nur partiell an den Inhalten und Gegenständen der beruflichen Fachrichtungen anschließt, was sich besonders in der Fachrichtung Wirtschaft und Verwaltung bemerkbar macht. Hier stellt sich nach wie vor die Frage, auf welches Fachverständnis von nachhaltigem Wirtschaften, respektive auf welche Theorie und Praxis der nachhaltigen Unternehmung die BBNE konkret bezogen werden kann. Das Ausbleiben einer Antwort auf diese Frage führt dazu, dass sich die BBNE auch zukünftig nicht in die berufsfeldspezifischen Handlungsfelder, d. h. Geschäftsprozesse und Aufgaben integrieren lassen wird. Es ist daher zwingend notwendig, dass die BBNE fachwissenschaftlich und fachpraktisch unterlegt wird.

Als Anknüpfungspunkt für die fachwissenschaftlich sowie fachpraktische Unterlegung einer BBNE kann der seit Jahrzehnten geführte Diskurs zum nachhaltigen Wirtschaften genutzt werden. Für die BBNE bietet dieser Diskurs die Chance, die Lehr-Lernangebote wissenschaftlich zu fundieren und vor allem an lebensweltlichen Zukunftspraktiken zu orientieren.

Neben dem Konzept der suffizienten Geschäftsmodelle (Schneidewind 2012), der Theorie einer kulturalistischen Unternehmung (Pfriem 2004, 2015, 2016; Beschorner 2015) und dem Ansatz neuer Wertschöpfungsmuster (Redlich \& Moritz 2018, 3) lassen sich eine Reihe weiterer Arbeiten benennen, die nachhaltiges Wirtschaften einordnen und modellieren als: (1) Antriebsfeder für die Innovationsgestaltung in Unternehmen (Fichter \& Clausen 2016); (2) integralen Bestandteil unternehmerischer Entscheidungen (vgl. Müller-Christ 2014); (3) Sustainable Supply Chain Management-Aufgabe (vgl. Seuring 2011) oder (4) als Triebfeder einer zukunftsgerechten, nachhaltigen Personalund Organisationsentwicklung (Ehnert, Harry \& Zink 2014).

Nachfolgend werden die ersten drei genannten Ansätze als Referenzpunkte einer fachwissenschaftlichen Fundierung der BBNE hinsichtlich ihrer Grundaussagen und Annahmen zum nachhaltigen Wirtschaften sowie ihrer Relevanz für die BBNE vorgestellt.

Konzept der Geschäftsmodellentwicklung

Geschäftsmodelle beschreiben die Kausallogiken, nach denen Unternehmen ihre Gewinnziele erreichen. Dazu werden Kundensegmente und -beziehungen, Leistungs- und Nutzenversprechen, Ressourcen, Fähigkeiten und Prozesse, Partner sowie das finanzielle Kalkül eines Unternehmens im Zusammenhang modelliert (Schneidewind 2012). 
Schneidewind illustriert, dass auch nachhaltig-suffiziente Geschäftsmodelle rentabel sind. Die Suffizienzstrategie zielt nach Stengel darauf ab, „dass Menschen ihr Verhalten ohne Zwang verändern und Praktiken, die Ressourcen übermäßig verbrauchen, einschränken oder ersetzen. Sie bemüht sich um einen genügsamen, umweltverträglichen Verbrauch von Energie“ (2012, 140).

Suffiziente Geschäftsmodelle bieten damit eine Alternative zu wachstumsorientierten Geschäftsmodellen. Statt auf Konsumquantität wird auf Konsumqualität gesetzt. Es geht um Befähigung mündiger Kunden und nicht darum, zusätzliche Bedürfnisse in bereits gesättigten Märkten zu wecken. Suffiziente Geschäftsmodelle zielen also auf die Entkopplung der Lebensqualität vom Ressourcenverbrauch ab. Sie setzen auf Entschleunigung, Entflechtung und Entrümpelung von Märkten, Konsumgewohnheiten und Lebensstilen. Geschäftsmodelle bieten dabei zunächst „nur“ eine konzeptuelle Basis, um Lernenden in der kaufmännischen Berufsbildung basale Zusammenhänge und vor allem Möglichkeiten und Zukunftsoptionen einer nachhaltigen Unternehmung aufzuzeigen.

Kulturalistische Theorie der nachhaltigen Unternehmung

Dieser zweite Referenzpunkt ist von den Arbeiten von Pfriem (2004, 2015, 2016) zur kulturalistischen Unternehmenstheorie und von Beschorner (2015) zur kulturalistischen Wirtschaftsethik geprägt. Mit kulturalistisch ist hier gemeint, dass es kein Jenseits von Kultur gibt. Der mögliche Ort nachhaltigen Wirtschaftens ist demnach jene kulturelle Praxis, die durch Unternehmensstrategien hervorgebracht und gestaltet wird. Die Grundannahme ist: „Unternehmensstrategien sind kulturelle Angebote an die Gesellschaft" (Pfriem 2004, 375)

Im Kern geht es darum, dass Unternehmen sich als gesellschaftliche Akteure begreifen, die durch ihre Art zu wirtschaften die Gesellschaft in einem nachhaltigen Sinne mitgestalten können. Es gilt hierfür, den Blick nicht allein auf Betriebsabläufe zu richten, sondern eine gesellschaftswissenschaftliche Perspektive einzunehmen und die Folgen dessen, was durch die Unternehmen in die Welt gesetzt wird, zu berücksichtigen. Es handelt sich um einen übergeordneten Ansatz, der ein grundständiges Nachdenken darüber und Aushandeln dessen, was Unternehmen wann, wie und warum tun, einfordert und eine umfassende Theorie der Unternehmung darstellt. Nachhaltiges Wirtschaften ist dabei nicht als Tatsache zu begreifen, die nur so und niemals anders möglich ist und bei der von vornerein festgelegt wäre, durch welche Strategie sie nun umzusetzen sei. Nachhaltiges Wirtschaften ist aus dieser Blickrichtung eine Frage gesellschaftlicher Verantwortung von Unternehmen. Moralische Werte und Verantwortung entstehen in dieser Perspektive durch gesellschaftliche Diskurse (Beschorner 2015) und soziale Praktiken (Pfriem 2015). Für die Berufsbildung besteht der Wert einer kulturalistischen Theorie der nachhaltigen 
Unternehmung darin, dass sie Lernenden, jenseits von Eindeutigkeiten, befähigt, sich als politische Wesen herauszubilden. Sie können lernen, mit Widersprüchen umzugehen und ein kritisches Verhältnis zur gesellschaftlichen Gegenwart zu entwickeln sowie zu der Rolle, die Unternehmen dabei spielen.

\section{Nachhaltiges Wertschöpfungsmanagement}

Als dritter Referenzpunkt wird hier die konkrete Gestaltung der Prozesse nachhaltigen Wirtschaftens thematisiert. Gemeint sind damit Arbeiten zur Bottomup-Ökonomie und zu neuen Wertschöpfungsprozessmustern. So hat sich inzwischen eine interdisziplinäre Community etabliert, die den Paradigmenwechsel von der industriellen Produktion hin zur Bottom-up-Ökonomie beforscht.

Redlich und Moritz beschreiben diesen Wechsel wie folgt: „Weltweit können wir beobachten, wie Menschen sich vernetzen und (weitestgehend) frei von Hierarchie und Markt und in der Regel unentgeltlich mit oder ohne Unternehmensbeteiligung gemeinsam Wert schöpfen (auch bekannt als commonsbased peer production). Neue Wertschöpfungsmuster sind dadurch entstanden, die entgegen traditioneller Konzepte auf Offenheit basieren und kollaborativer sowie dezentraler Natur sind (zusammengefasst im Konzept der Bottom-upÖkonomie). Wir sehen ein neues erstarktes Selbstverständnis von Konsumenten, die zunehmend auch zu Produzenten, sogenannten „Prosumern“, werden können und nicht mehr zwingend auf Unternehmen angewiesen sind. Die professionelle und nicht-professionelle Sphäre verschwimmen. Somit verändert sich auch die Rolle von Unternehmen: Vom ehemals zentralen und beherrschenden Akteur degradiert zu einem untergeordneten Teil eines Systems. Sharing-Plattformen ermöglichen es Privatpersonen, selbst als Wertschöpfungsakteure tätig zu werden (sharing economy).“ (2018, 3).

Zusammengefasst wird damit nicht nur aufgezeigt, wie nachhaltige Wertschöpfung auf Basis suffizienter Geschäftsmodelle und vor dem Hintergrund nachhaltiger Kulturen der Unternehmung entstehen könnte, sondern vor allem auch, welche Mitgestaltungschancen sich in Beruf und Arbeit für Individuen ergeben könnten. Konkret umgesetzt wird dieser Ansatz bereits heute in sogenannten offenen Werkstätten.

Die Erklärungs- und Gestaltungsansätze zum nachhaltigen Wirtschaften stehen dabei weitestgehend unverbunden nebeneinander. Der Modellversuch GeKoNaWi hat den Versuch gewagt, für die BBNE anschlussfähige Referenzpunkte auszuwählen, zu prüfen und zu erproben. Die Fragestellung lautete: Wie können betriebliche Ausbilder zum einen fachtheoretisch und fachpraktisch im Hinblick auf die Theorie und Praxis des nachhaltigen Wirtschaftens sowie zum anderen wirtschaftspädagogisch und wirtschaftsdidaktisch mit Bezug zur Theorie und Praxis der Kompetenzentwicklung professionalisiert werden? 
Zur Beantwortung dieser Frage wurde die Professionalisierung des betrieblichen Ausbildungspersonals in ihren idealtypischen Wirkungszusammenhängen und Bruchstellen untersucht. Es wurden zunächst (1) curricular-didaktische Bezüge zur Fachwissenschaft und Fachpraxis des nachhaltigen Wirtschaftens hergestellt, (2) betrieblich-berufliche Handlungsmuster des nachhaltigen Wirtschaftens ausgewiesen, (3) Konzepte einer BBNE-Didaktik entworfen und (4) ein Kompetenzstrukturmodell entwickelt. Diese theoretisch-konzeptuellen Befunde wurden in Form einer Modulfortbildung für das betriebliche Ausbildungspersonal in den Domänen Einzel-, Groß- und Außenhandel erprobt und evaluiert. ${ }^{2}$

Im Projekt wurde u. a. die von Osterwalder und Pigneur (2011) vorgelegte CANVAS-Methode zur Geschäftsmodellentwicklung zu einem neuen Instrument (Gemo.NaWi) ${ }^{3}$ weiterentwickelt: Dort werden die Anforderungen nachhaltig-suffizienter Geschäftsmodelle durch sechszehn Leitfragen zu den Dimensionen Finanzen, Nutzen, Kunden, Wertschöpfung, Partner und Unternehmensumwelt abgebildet (hierzu Kiepe et al. 2019, 34). Ziel ist es, den Entwicklungsprozess von Geschäftsmodellen umfassend und verständlich darzustellen. Die einzelnen Fragen haben einen gegenseitigen Bezug. Die Anwender der Gemo.NaWi werden mit der Komplexität der Geschäftsmodellentwicklung konfrontiert, zugleich wird diese aber auch gestaltbar. Insbesondere aber löst die Arbeit mit der Gemo.NaWi einen kreativen, zukunftsoffenen und multiperspektivischen Diskurs aus. Aus der Warte der betrieblich-beruflichen Bildung stellt das Konzept der Geschäftsmodelle einen effektiven Lerngegenstand dar: Es lassen sich Zugänge zu komplexen Zusammenhängen eröffnen, Lernende an zentralen Zukunftsfragen beteiligen und Aufgaben der Mitgestaltung ableiten. Es werden ganz konkret die Handlungsalternativen, Zusammenhänge und Wirkungen visualisiert. Damit werden unternehmerisches, systemisches und prozessuales Denken und Handeln gefördert. Zugleich wird ein „Schonraum“ geboten, in dem Handlungsalternativen in den Berufsdomänen durchdacht, modelliert und diskutiert werden können.

2 Die fünf zukunftsorientierten Lerngegenstände der Modulfortbildung wurden von insgesamt 87 Teilnehmer*innen erprobt. Die Erprobung erfolgte in unterschiedlichen Formaten. Diese reichen von Inhouseschulung, über Fortbildungen bei der IHK und Verbänden, bis hin zur Erprobung in einem 3D-Learnspace.

3 Alle Materialien und Anleitungen zur Modulfortbildung sind als Open Access verfügbar: Kiepe, K., Wicke, C., Reichel, J., Schlömer, T., Becker, C., Jahncke, H. \& Rebmann, K. (2019). Geschäftsmodell- und Kompetenzentwicklung für nachhaltiges Wirtschaften. Handbuch und Fortbildungskonzept für die betriebliche Personalentwicklung. Berlin: Logos. 


\section{3. „Systemische Visualisierung“ als Erkenntnismethode zum Umgang mit Widersprüchlichkeiten zwischen Wachstumsorientierung und Nachhaltigkeitsorientierung am Beispiel des Modellversuchs Pro-DEENLA}

Wendet man den Blick von der im letzten Abschnitt erörterten fachwissenschaftlichen Fundierung einer BBNE auf die Ebene des Subjekts, wird deutlich, dass beruflich Lernende dazu herausgefordert sind, ihre Persönlichkeit im Umgang mit Widersprüchlichkeiten zwischen einer fachwissenschaftlich fundierten Nachhaltigkeitsidee und ihren alltäglichen betrieblichen Lebenssituationen zu entwickeln. Denn nachhaltigkeitsorientierte berufliche Handlungen zeichnen sich durch Situationen aus, in denen Entscheidungen zwischen Alternativen getroffen werden müssen, die mitunter nicht zur gleichen Zeit realisiert werden können (exemplarisch Fischer, Hahn \& Hantke 2017).

Dies rekurriert auf den bereits im letzten Abschnitt erwähnten ressourcenorientierten ${ }^{4}$ Managementansatz von Müller-Christ (2014), der Nachhaltigkeit als Prämisse eines rationalen Wirtschaftens betrachtet. Kurz: Müller-Christ sieht nachhaltiges Wirtschaften als die ,Erhaltung der ökologischen, ökonomischen und sozialen Substanz [an], aus der die Gesellschaft dauerhaft wirtschaften will“ $(2014,205)$ und betont damit, dass genau genommen das ,durch Nachhaltigkeit zu lösende Problem ein originär ökonomisches ist: das Haushalten mit Ressourcen" (ebd., 115). Im Kontext des gegenwärtigen Wirtschaftens, das „neben unbestrittenen Erfolgen zu ökologischen Zerstörungen, sozialen Verwerfungen und immer wiederkehrenden ökonomischen Krisen“ (Schneidewind et al. 2016, 1) führt, kann die Nachhaltigkeitsidee jedoch als Kritik an eben diesen negativen Entwicklungen angesehen werden.

Zur Übertragung dieser gesellschaftspolitischen Perspektive auf die Ebene des beruflichen Alltags, ist es hilfreich, sich die Prämissen anzuschauen, die für ein (nachhaltig ausgerichtetes) berufliches Handeln grundsätzlich relevant sind. Demnach muss das komplexe berufliche Handeln funktionieren (Funktionalität), sich rechnen (ökonomische Effizienz), legal sein (Legalität), ökologisch verträglich sein (ökologische Effizienz), sozial verantwortbar sein (Sozialverträglichkeit), auf Dauer angelegt sein (Substanzerhaltung) und verantwortungsethisch „einwandfrei“ sein (Verantwortung) (Müller-Christ 2014, 361). Da die Beziehungen zwischen diesen Prämissen auf der Restriktion absolut knapper Ressourcen beruhen, gestalten sich diese mitunter widersprüchlich.

4 Müller-Christ (2014, 305 ff.) geht bei der Verwendung des Ressourcenbegriffs im Sinne der Nachhaltigkeitsdimensionen von natürlichen, gesellschaftlichen sowie wirtschaftlichen Ressourcen aus. 
An dieser Stelle setzte der Modellversuch „Pro-DEENLA“ an. Im Rahmen von Pro-DEENLA wurden gemeinsam mit 15 Praxispartnerbetrieben 27 Lernaufgaben für den Einsatz in der betrieblichen Ausbildung zum Kaufmann / zur Kauffrau für Spedition und Logistikdienstleistung konzipiert, erprobt und verbreitet. Der konstruktive, domänenspezifische Umgang mit den Widersprüchlichkeiten zwischen einer effizienz- und wachstumsorientierten Unternehmenspolitik und einer gesellschaftspolitischen, sozialen und ökologischen Verantwortungsethik kann durch diese Lernaufgaben - teilweise implizit - gefördert werden (Hantke \& Pranger 2019). Ziel dieses Prozesses - wie auch des gesamten Modellversuchsförderschwerpunkts - war die Persönlichkeitsentwicklung beruflich Lernender im Sinne der Förderung einer umfassenden, nachhaltigkeitsorientierten beruflichen Handlungskompetenz.

Eine Möglichkeit, das Spannungsfeld zwischen betrieblichen Anforderungen und nachhaltigen Herausforderungen didaktisch zu inszenieren, ist der Einsatz der im Rahmen von Pro-DEENLA erprobten und als Lernmethode reformulierten Erkenntnismethode „Systemische Visualisierung“, die ihren Ursprung in der systemischen Therapie hat und sich mittlerweile in der Managementberatung etabliert hat (Müller-Christ \& Pijetlovic 2018, 279). Ziel der Anwendung dieser Methode ist es, durch eine Übersetzung von einer Text- in eine Raum-, Körper- und Bildsprache, informelle Strukturen und Beziehungen aufzudecken und deren komplexe Geflechte reflexiv zugänglich zu machen. In diesem Prozess wird nicht nur die kognitive Ebene der Lernenden angesprochen, sondern auch Emotionen und Intuitionen der Lernenden bewusst adressiert (Fischer et al. 2018).

Konkret wird im Rahmen der „Systemischen Visualisierung“ sukzessive ein Raumbild entwickelt, indem Personen als Stellvertretende von zuvor bestimmten Elemente im Raum positioniert werden. Die Entstehung des Raumbildes wird von den Stellvertretenden verbalisiert und gemeinsam mit den möglichen Zuschauenden interpretiert und reflektiert. „Systemische Visualisierungen" sind also dynamisch ausgerichtet - das interaktive und kommunikative Vorgehen steht im Mittelpunkt. Dabei sollen die Lernenden a) das Bild selbst konstruieren und b) das dreidimensionale Bild betrachten und interpretativ nachvollziehen, wodurch das analytische Denken mit dem interpretativen Denken und das kausale Denken mit dem Strukturdenken verknüpft wird. Damit baut der Prozess der „Systemischen Visualisierung“ eine gemeinsame Gesprächsgrundlage auf. Auf Basis dieser Grundlage kann ein tieferes Verständnis für den visualisierten Sachverhalt aufgebaut werden. 
Abb. 1: Mögliche „Systemische Visualisierung “ zum komplexen Beziehungsgeflecht der (nachhaltig ausgerichteten) Transport- und Logistikbranche

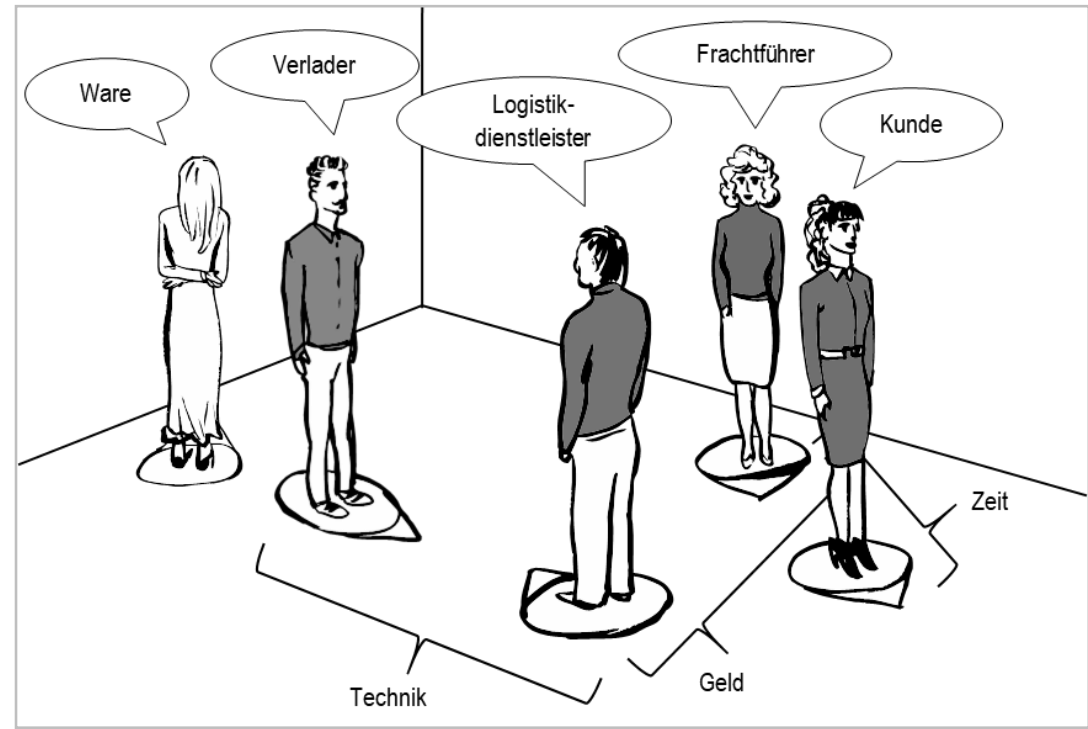

Quelle: eigene Darstellung

Das im Rahmen der „Systemischen Visualisierung“ sukzessiv entstehende Raumbild kann wie folgt gedeutet werden (Müller-Christ \& Pijetlovic 2018, 279):

- Sind die Elemente einander zugewandt, sind sie sehr stark auf einander bezogen, stehen im Konflikt miteinander oder haben ein bestimmtes Anliegen zu klären.

- Ist nur ein Element dem anderen zugewandt, handelt es sich um eine einseitige Beziehung.

- Die Hauptblickrichtung zeigt an, auf wen oder was sich ein Element bezieht. Dabei kann es andere Elemente im Blickfeld haben, sie aber nur am Rande wahrnehmen.

- Je größer der Abstand zwischen den Elementen, desto größer ist auch die Perspektive der Elemente. Großer Abstand kann demnach eine schwache Beziehung andeuten, muss es aber nicht. 
Die Ausbilder*innen ${ }^{5}$ der „Pro-DEENLA“-Praxispartnerbetriebe haben beispielsweise das komplexe Beziehungsgeflecht der (nachhaltig ausgerichteten) Transport- und Logistikbranche sowie ihre persönliche Nachhaltigkeitswahrnehmung visualisiert (Abb. 1). Dabei hat die Visualisierung verdeutlicht, dass die Transport- und Logistikbranche einem enormen technischen, finanziellen und zeitlichen Effizienzdruck ausgesetzt ist, der oftmals im Widerspruch zur Realisierung eines sozial gerechten Lebens in einer intakten Umwelt steht. In der Diskussion merkten die Ausbilder*innen an, dass eine Ausweitung betrieblicher Nachhaltigkeit in der Regel über betriebswirtschaftlich-kaufmännische Argumentationsfiguren gerechtfertigt werden müsse. Vor diesem Hintergrund plädierten sie dafür, Nachhaltigkeit nicht als Belastung, sondern als Kern betrieblicher Zukunftsfähigkeit aufzufassen. Dazu gehöre auch, betriebliche „Reflexionsräume“ - wie die Durchführung der „Systemischen Visualisierung“ zu schaffen, um die sozialen und ökologischen Auswirkungen des eigenen Handelns (nicht nur) im Betrieb sichtbar und reflexiv zugänglich machen $\mathrm{zu}$ können. Nicht zuletzt an diesem Beispiel wird deutlich, dass sich mit Hilfe der Erkenntnismethode „Systemische Visualisierung“ die oben skizzierten, scheinbar unvereinbaren Leitbilder einer effizienz- und wachstumsorientierten Unternehmenspolitik auf der einen und einer gesellschaftspolitischen, sozialen und ökologischen Verantwortungsethik auf der anderen Seite bildungswirksam inszenieren lassen. Denn das Spannungsverhältnis zwischen einer Orientierung an der langfristig ausgerichteten Nachhaltigkeitsidee und der überwiegend kurzfristig ausgerichteten Wachstumssteigerung eröffnet Räume, die es erlauben, unterschiedliche Vorstellungen, Prämissen, Weltbilder und Leitlinien kritisch-konstruktiv zu reflektieren und gegebenenfalls (neu) zu erfinden. Kurzum: Die im Rahmen von Pro-DEENLA erprobte Erkenntnismethode „Systemische Visualisierung“ hilft, in einem ästhetisch-performativen Prozess die Veränderung der Wahrnehmung zu ermöglichen und Alternativen aufzudecken. Dabei handelt es sich um einen resonanten Erkenntnisprozess, der ein (selbst-)reflexives Denken und Handeln in multiplen Ursache-Wirkungs-Zusammenhängen ermöglicht. Somit können die Multikausalitäten zwischen einer fachwissenschaftlich fundierten Nachhaltigkeitsidee und den alltäglichen betrieblichen Lebenssituationen kognitiv und emotional erfasst und im beruflichen Handlungsalltag problemlösend verarbeitet werden, um vom Erkennen und Lernen zum tatsächlichen Handeln bzw. Gestalten zu gelangen (Hantke 2018).

5 Im Rahmen von Pro-DEENLA wurden Methodenkarten entwickelt, die Ausbilder*innen beim Einsatz der „Systemischen Visualisierung“ behilflich sein können. Online: http://bwpschriften.univera.de/Band20_18/02a_fischer_hantke_roth_senneke_pranger_Band20_18.pdf Die grundsätzliche und berechtigte Frage nach der weiteren Professionalisierung des Berufsbildungspersonals im Umgang mit der Methode „Systemische Visualisierung“ kann an dieser Stelle nicht ausführlich erörtert werden (im Hinblick auf Professionalisierung in der Durchführung von Systemaufstellungen St. John 2019). 


\section{Ein Fazit und Ausblick: „Lebendiges Lernen“ als balancierendes didaktisches Prinzip der BBNE}

Die aufgezeigten Beispiele illustrieren ein Kernanliegen der BBNE, gewissermaßen ihre „DNA“ (Fischer et al. 2020): Den Umgang mit Widersprüchlichkeiten, beruflichen Paradoxien und konkurrierenden unternehmerischen und gesellschaftlichen Interessen. In der Gesamtschau des Modellversuchsprogramms wurde deutlich, dass alle didaktischen Konzepte in unterschiedlichen Ausprägungen darauf abzielten, Räume zu schaffen - für die Exploration und Aushandlung von Widersprüchen, für das Denken in Alternativen, für Gestaltung und Transformation. Solche auf Reflexion zielende Räume können analytisch sein, wie bei der Geschäftsmodellentwicklung von GeKoNaWi, oder physisch repräsentierend, wie bei Pro-DEENLA. Sie werden "Schonräume“, „Resonanzräume“, „Denk-Räume“ oder „Gedeihräume“ genannt. Mit derartigen Metaphern des „Gedeihens“ wird organisches Wachstum und lebendiges Lernen assoziiert, wobei „Lebendiges Lernen“ im Sinne der humanistischen Psychologie mehr ist als eine Metapher, nämlich ein fester Begriff mit methodischen Zugängen (Cohn 2016; Cohn \& Terfurth 2007; Härle 2011; Stollberg \& Schneider-Landolf 2010). Das „Lebendige“ hat hierbei eine dreifache Bestimmung (Casper 2019): (1) indem sich Lernprozesse auf konkrete Biographien, Lebensentwürfe und subjektiv relevante Lebenswelten beziehen; (2) indem die Prozesse eine lebendige, das heißt: aktivierend-sinnliche Qualität mit emotionaler Tiefe aufweisen und (3) indem Lernprozesse auf unmaskiert-authentische Begegnungen abzielen, in denen Ehrfurcht vor dem Gegenüber und dem Lebendigen an sich zum Tragen kommen.

Im Sammelband zu diesem Modellversuchsprogramm werden insgesamt zehn Handlungsorientierungen zur didaktischen Gestaltung der BBNE hergeleitet (Schütt-Sayed et al. im Erscheinen), im Rahmen dieser theoretischen Reflexion soll exemplarisch nur das Prinzip des „Lebendigen Lernens“ hervorgehoben werden. Es erlangt seine besondere Bedeutung für die BBNE dadurch, dass es in Anlehnung an Härle (2011) als Gegenpol des für die Berufsbildung unstrittigen Prinzips der „Kompetenzorientierung“ verstanden werden kann. Wo es der Kompetenzorientierung um Definition, Rationalität und Struktur geht, definiert sich das Konzept des „Lebendigen Lernens“ über deren „Schwestertugenden“ von Metapher, Emotionalität und Improvisation. Beide Prinzipien können entwertend übertrieben werden - so führt überspitzte Definition zu Reduktion, Rationalität möglicherweise zu überhöhter Verzweckung und unreflektierte Strukturen gar zu Starrheiten. Auf dieser Seite der Überspitzungen lassen sich eingetretene unternehmerische Pfade, festgefahrene Prozesse und daraus geborene Sachzwänge und Betriebsblindheiten verorten. Auswege aus solchen Dynamiken bieten nun ebenjene Merkmale des „Lebendigen Lernens“, realisiert z. B. in den oben genannten Räumen zum Denken 
und Fühlen in Alternativen. Wenn BBNE als transformativer Prozess im Sinne der regulativen Idee der Nachhaltigkeit greifen soll, sind es genau solche Prozesse des gedanklichen und emotionalen Öffnens, des kreativen Umdenkens und der beruflichen wie privaten Partizipation. Doch auch Metapher, Emotionalität und Improvisation können entwertend übertrieben werden, in ein esoterisch anmutendes ,,anything goes“ aus Ubiquität, Irrationalität und Willkür. Es gilt daher, für eine balancierte BBNE im Dienst der Lernenden, „Lebendiges Lernen“ und „Kompetenzorientierung“ als Schwesterprinzipien zu verstehen, die in einem positiven Spannungsverhältnis gehalten werden sollten, um Übertreibungen und Sackgassen sowie Verzweckung oder Verflachung der beruflichen Bildung zu vermeiden. Nach dem Vorbild von Schulz von Thun (2018) lässt sich dieser Zusammenhang in einem Werte- und Entwicklungsquadrat darstellen, das Leitplanken für eine balancierte didaktische Gestaltung bietet:

Abb. 2: Werte- und Entwicklungsquadrat für eine Didaktik der BBNE

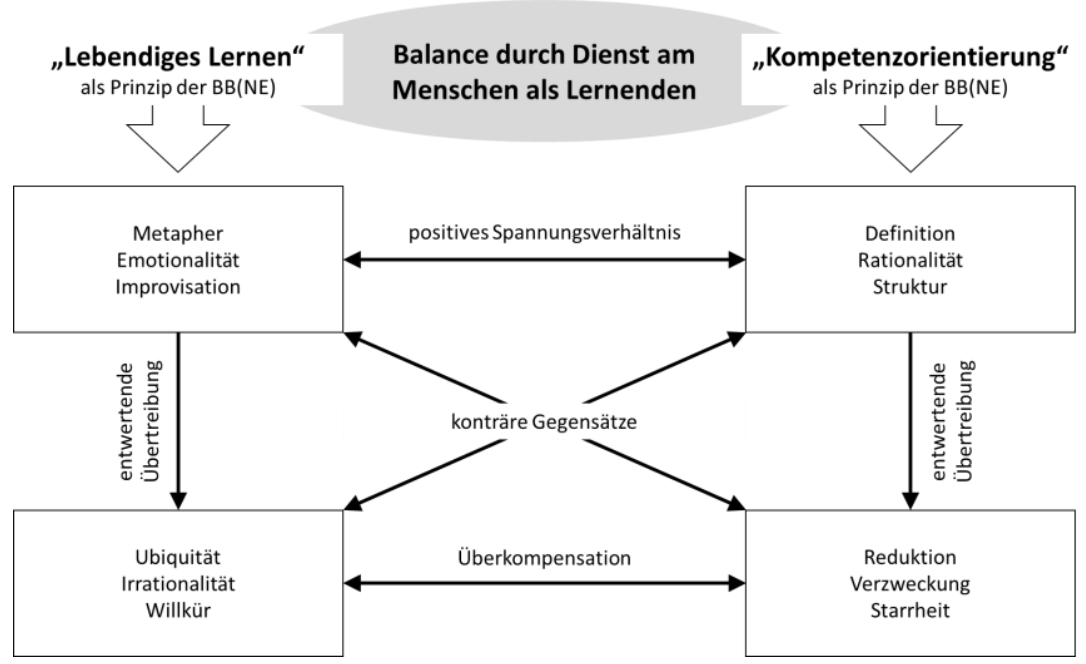

Quelle: eigene Darstellung in Anlehnung an Casper 2019; Schulz von Thun 2018

So wie es im öffentlichen Diskurs nachhallt, kann sich eine nachhaltige Entwicklung weder in Form eines krisenhaften Katastrophenszenarios noch einer boulevardjournalistischen „Ökodiktatur“ durchsetzen. Sie kann sich weder der Verzweckung für althergebrachte Strukturen unterwerfen, noch sich in träumerischen Illusionen verlieren, wenn sie ihr transformatives Anliegen verfolgen will. Nicht nur, aber besonders in der beruflichen Bildung gilt es vielmehr, 
Räume für das Denken in Alternativen und die berufsfachlich fundierte Aushandlung von Widersprüchen zu schaffen, wie es an den Beispielen der Modellversuche verdeutlicht wurde.

Die didaktische Implikation, und damit auch das unmittelbare Desiderat für weitere Forschung, liegt in der Balance zwischen für sich genommen unstrittigen, aber sich notwendigerweise in positiver Spannung befindenden Prinzipien. Didaktische Forschung zur BBNE sollte sich demnach weiter mit der Identifikation von Spannungsfeldern als potenzielle Lerngegenstände beschäftigen und das methodische Repertoire zu deren produktiver Aushandlung erweitern, sowie dieses durch Wirkungsforschung elaborieren.

Im Fazit lebt die Didaktik der BBNE von der Dynamik von Widerspruch und Balance, im Spannungsfeld beruflicher Sachkompetenz und Sachzwänge einerseits und humanistischer Werte und Anliegen andererseits. Dieses der Berufsbildung so immanente Spannungsfeld wurde von Cohn emotiv auf den Punkt gebracht: „Achtung vor dem Leben ist wichtig. Fähigkeiten und Wissen sind wichtig. Wissen ohne Achtung vor den Menschen baut Gaskammern und Napalmfabriken. Menschlichkeit ohne Wissen kann kein Brot backen, keine Häuser, Spitäler oder Schulen bauen und keine gebrochenen Knochen oder Seelen heilen.“ (Cohn 2016, 109)

\section{Literatur}

Beschorner, T. (2015). Kulturalistische Wirtschaftsethik. Grundzüge einer Theorie der Anwendung. In D. van Aaken \& P. Schreck (Hrsg.), Theorien der Wirtschafts- und Unternehmensethik (S. 151-180). Berlin: Suhrkamp.

Casper, M. (2019). Lebendige Wirtschaftsdidaktik. Problemfelder, Entscheidungsfelder und Forschungsfelder der Wirtschaftsdidaktik am Beispiel des Prototyps ,,Wirtschaftswissenschaften als Gegenstand Ökonomischer Bildung “. Dissertation. Universität Hamburg, Hamburg.

Cohn, R. C. (2016). Von der Psychoanalyse zur themenzentrierten Interaktion. Von der Behandlung einzelner zu einer Pädagogik für alle (18. Aufl.). Stuttgart: KlettCotta (Konzepte der Humanwissenschaften).

Cohn, R. C. \& Terfurth, C. (2007). Lebendiges Lehren und Lernen. TZI macht Schule (5. Aufl.). Stuttgart: Klett-Cotta.

Ehnert, I., Harry, W. \& Zink, K. J. (2014). Sustainability and Human Resource Management. Berlin: Springer.

Engartner, T., Fridrich, C. \& Graupe, S. (2018). Sozioökonomische Bildung und Wissenschaft. Entwicklungslinien und Perspektiven. Wiesbaden: Springer Fachmedien.

Fichter, K. \& Clausen, J. (2016). Diffusion Dynamics of Sustainable Innovation - Insights on Diffusion Patterns Based on the Analysis of 100 Sustainable Product and Service Innovations. Journal of Innovation Management, 2(4), 30-67. 
Fischer, A. et al. (2018). Lernmodul „Umgang mit Widersprüchen”. Berufsbildungswissenschaftliche Schriften, 20, 1-8. Online: http://bwp-schriften.univera.de/ Band20_18/02b_fischer_hantke_roth_senneke_pranger_Band20_18.pdf (27.11.2019).

Fischer, A., Hahn, G. \& Hantke, H. (2017). Gesucht: Resonanzräume für Wahrnehmung und Erkennen in der Berufsbildung für nachhaltige Entwicklung sowie in der sozio-ökonomischen Bildung. Vom „Wahrnehmen-Müssen“ zum „Mehrwahrnehmen-Können“. Berufsbildungswissenschaftliche Schriften, 18, 4-32. Online: http://bwp-schriften.univera.de/Band18_17/03_fischer_hahn_hantke_Band 18_17.pdf (27.11.2019).

Fischer, A., Hantke, H. \& Roth, J.-J. (2020, im Erscheinen). Innovatives Lernen zwischen betrieblichen Anforderungen und nachhaltigen Herausforderungen. In Melzig et al. (Hrsg.), Berufsbildung für nachhaltige Entwicklung 2015 - 2019. Berichte zur beruflichen Bildung. Bonn.

Härle, G. (2011). „Lebendiges Lernen“ und „Kompetenz“ - Gegensatz oder Bereicherung? Themenzentrierte Interaktion, 25(2), 11-24.

Hantke, H. (2018). „Resonanzräume des Subpolitischen“ als wirtschaftsdidaktische Antwort auf ökonomisierte (wirtschafts-)betriebliche Lebenssituationen - eine Forschungsheuristik vor dem Hintergrund der Nachhaltigkeitsidee. bwp@Berufsund Wirtschaftspädagogik - online, 35, 1-23. Online: http://www.bwpat.de/ausgabe35/hantke_bwpat35.pdf (27.11.2019).

Hantke, H., \& Pranger, J. (2019). Nachhaltigkeits-Werte ausbilden und kommunizieren: Lernmodule zu Corporate Social Responsibility im Bereich Transport und Logistik. Berufsbildung in Wissenschaft und Praxis - BWP, 48(4), 29-31.

Hemkes, B. (2014). Vom Projekt zur Struktur - Das Strategiepapier der AG „Berufliche Aus- und Weiterbildung“. In W. Kuhlmeier, T. Vollmer \& A. Mohorič (Hrsg.), Berufsbildung für nachhaltige Entwicklung. Modellversuche 2010-2013: Erkenntnisse, Schlussfolgerungen und Ausblicke (S. 235-255). Bertelsmann W. Verlag (Berichte zur beruflichen Bildung).

Kastrup, J., Kuhlmeier, W., Reichwein, W. \& Vollmer, T. (2012). Mitwirkung an der Energiewende lernen - Leitlinien für die didaktische Gestaltung der Berufsbildung. lernen \& lehren, 107, 117-124.

Kiepe, K., Wicke, C., Reichel, J., Schlömer, T., Becker, C., Jahncke, H. \& Rebmann, K. (2019). Geschäftsmodell- und Kompetenzentwicklung für nachhaltiges Wirtschaften. Handbuch und Fortbildungskonzept für die betriebliche Personalentwicklung. Berlin: Logos.

Kremer, H.-H., Tramm, T. \& Wilbers, K. (2014). Kaufmännische Bildung? Sondierungen zu einer vernachlässigten Sinndimension. Berlin: epubli GmbH (Texte zur Wirtschaftspädagogik und Personalentwicklung, 10).

Kuhlmeier, W., Vollmer, T. \& Mohorič, A. (2014). Berufsbildung für nachhaltige Entwicklung. Modellversuche 2010-2013: Erkenntnisse, Schlussfolgerungen und Ausblicke (1. Aufl.). Bertelsmann W. Verlag (Berichte zur beruflichen Bildung).

Müller-Christ, G. \& Pijetlovic, D. (2018). Komplexe Systeme lesen. Das Potential von Systemaufstellungen in Wissenschaft und Praxis. Berlin: Springer.

Müller-Christ, G. (2001). Nachhaltiges Ressourcenmanagement. Eine wirtschaftsökologische Fundierung. Marburg: Metropolis. 
Müller-Christ, G. (2014). Nachhaltiges Management. Einführung in Ressourcenorientierung und widersprüchliche Managementrationalitäten (2. Aufl.). Baden-Baden, Stuttgart: UTB.

Osterwalder, A. \& Pigneur, Y. (2011). Business Model Generation. Ein Handbuch für Visionäre, Spieleveränderer und Herausforderer. Frankfurt a. M.: Campus

Pfriem, R. (2004). Unternehmensstrategien sind kulturelle Angebote an die Gesellschaft. In R. Pfriem (Hrsg.), Perspektiven einer kulturwissenschaftlichen Theorie der Unternehmung (S. 375-404). Marburg: Metropolis.

Pfriem, R. (2015). Kulturalistische Unternehmensethik. In D. van Aaken \& P. Schreck (Hrsg.), Theorien der Wirtschafts- und Unternehmensethik (S. 187-208). Berlin: Suhrkamp.

Pfriem, R. (2016). Ökonomie als kulturelle Praxis. In R. Pfriem (Hrsg.), Ökonomie als Gemengelage kultureller Praktiken (S. 19-34). Marburg: Metropolis.

Redlich, T. \& Moritz, M. (2018). Die Zukunft der Wertschöpfung - dezentral, vernetzt und kollaborativ. In T. Redlich, M. Moritz \& J. P. Wulfsberg (Hrsg.) Interdisziplinäre Perspektiven zur Zukunft der Wertschöpfung (S. 1-8). Wiesbaden: Springer Gabler.

Rhein, R. (2015). Hochschulisches Lernen - eine analytische Perspektive. Zeitschrift für Weiterbildungsforschung - Report, 38(3), 347-363.

Schneidewind, U. et al. (2016). Transformative Wirtschaftswissenschaft im Kontext nachhaltiger Entwicklung. Ökologisches Wirtschaften - Fachzeitschrift, 31(2), 3034.

Schneidewind, U. (2012). Nachhaltiges Ressourcenmanagement als Gegenstand einer transdisziplinären Betriebswirtschaftslehre. In H. Corsten \& S. Roth (Hrsg.), Nachhaltigkeit. Unternehmerisches Handeln in globaler Verantwortung (S. 6792). Wiesbaden: Springer Gabler.

Schütt-Sayed, S., Casper, M. \& Vollmer, T. (in Vorbereitung). Mitgestaltung lernbar machen - Didaktik der Berufsbildung für nachhaltige Entwicklung. In Melzig et al. (Hrsg.), Berufsbildung für nachhaltige Entwicklung 2015 - 2019. Berichte zur beruflichen Bildung. Bonn.

Schulz von Thun, F. (2018). Stile, Werte und Persönlichkeitsentwicklung. Differentielle Psychologie der Kommunikation (37. Aufl.). Reinbek: Rowohlt.

Seuring, S. (2011). Supply Chain Management for Sustainable Products - Insights from research applying mixed-methodologies. Business Strategy and the Environment, 20(7), 471-484.

St. John, D. (2019). Zur Didaktik der Aufstellungsarbeit. Zeitschrift für Psychodrama und Soziometrie, 18(1), 243-255.

Stollberg, D. \& Schneider-Landolf, M. (2010). Lebendiges Lernen. In M. SchneiderLandolf, J. Spielmann \& W. Zitterbarth (Hrsg.), Handbuch Themenzentrierte Interaktion (TZI) (2. Aufl., S. 147-153). Göttingen: Vandenhoeck \& Ruprecht.

World Commission on Environment and Development (WCED) (1987). Our Common Future: Report of the World Commission on Environment and Development. Doc. $\mathrm{A} / 42 / 427$. 



\title{
Verarbeitung von Studienabbrüchen innerhalb beruflicher Entwicklungsprozesse - eine berufsbiografische Analyse
}

\author{
Dana Bergmann
}

\section{Problemaufriss}

Bildungsmobilität stellt ein erklärtes Ziel bildungspolitischer Bemühungen dar (u. a. Hemkes \& Wilbers 2019; Frommberger 2019). Dabei geht es um die Anschlussfähigkeit von Akteuren an ihre Umgebung. Der Mobilitätsbegriff verschränkt zwei Analyseebenen: Auf struktureller Ebene geht es um Fragen nach der Erreichbarkeit, Zugangsmöglichkeit und Durchlässigkeit. Auf personaler Ebene kennzeichnet der Mobilitätsbegriff die individuelle Beweglichkeit zwischen (Bildungs-)Aktivitäten (Dick 2009).

Der strukturelle Mobilitäts- bzw. Durchlässigkeitsdiskurs verlagerte sich in den letzten Jahren auf Bewegungen ,nach unten“ (Jahn \& Birckner 2014, 14), sodass die Gruppe der „Studienabbrecher*innen“ zunehmend in den Fokus des Hochschul- und Berufsbildungssystems rückte. Da beide Mobilitätsebenen in einem Wechselverhältnis zueinander stehen (Dick 2009), erhöhen steigende Mobilitätserfordernisse auf struktureller Ebene die Anforderungen an individuelle berufliche Orientierungsleistungen (Bergmann 2020). Auch wenn, wie bspw. in den Empfehlungen des Wissenschaftsrates expliziert, nachgelagerte „Interessenverschiebungen oder persönliche Entwicklungen stattfinden“ und „Entscheidungsrevisionen bzw. Neuorientierungen“ (Wissenschaftsrat 2014) legitimiert werden, wird in den Diskursen deutlich, dass zwar die personale Ebene der Bildungsmobilität thematisiert wird, die mit diesen Übergängen verbundenen Probleme allerdings primär auf struktureller Ebene diskutiert werden. In Bezug auf Studienabbrüche führt die Problematik der Anrechenbarkeit und Zertifizierung der im Studium erworbenen Kompetenzen, die für den weiteren beruflichen Verlauf weder transparent noch individuell verwertbar sind (Jahn \& Birckner 2014), zu einer Einschränkung der strukturellen Mobilität. Die Wechselseitigkeit beider Mobilitätsebenen nährt die Vermutung, dass Studienabbrüche auch auf personaler Ebene problematisch sind. Um die personale Ebene zu untersuchen und zu erfahren, welche biografische Relevanz Studienabbrüche in beruflichen Lebensläufen ehemaliger Studierender einnehmen, ist es notwendig, eine subjektorientierte Perspektive einzunehmen. Es soll innerhalb dieses Beitrags geklärt werden, wie Studienabbrecher*innen ihren beruflichen Weg trotz eines diskontinuierlichen Verlaufs gestalten, 
welche subjektiven Deutungs- und Verarbeitungsmuster diesem zugrunde liegen und welche Bedeutung der Beruf innerhalb ihres Werdegangs einnimmt.

\section{Der Studienabbruch als Forschungsgegenstand}

Studienabbrüche stellen neben Studienwechseln (bspw. auf Fach- bzw. Hochschulebene) eine Ausprägung des Schwunds an Hochschulen dar (Heublein \& Wolter 2011). Als Studienabbrecher*innen, gelten ehemalige Studierende, die zwar durch Immatrikulation ein Erststudium an einer deutschen Hochschule aufgenommen haben, dann aber das Hochschulsystem endgültig ohne (erstes) Abschlussexamen verlassen haben“ (Heublein et al. 2017, 273). Die komplexe Thematik des Studienabbruchs wird multidisziplinär vorwiegend im Hinblick auf die Fragen nach dem Umfang, den Ursachen, den Folgen und Wirkungen sowie der Prävention erforscht, die jedoch nicht immer klar voneinander abgegrenzt werden können (Schröder \& Daniel 1998). Der Umfang wird zwar nicht bundes- und hochschulweit, allerdings in regelmäßigen Abständen vom Deutschen Zentrum für Hochschul- und Wissenschaftsforschung in Form von Studienabbruchquoten ermittelt (u. a. Heublein et al. 2017). So beträgt bspw. die Abbruchquote bei Bachelorstudiengängen an Universitäten durchschnittlich $32 \%$ (Heublein et al. 2018, 13). Aufgrund der vielfältigen Hochschul- und Abschlussarten sowie Fächergruppen ist die Aussagekraft einer allgemeingültigen Gesamtquote allerdings eingeschränkt (Bergmann 2016). In Bezug auf die Ursachen des Studienabbruchs lässt sich konstatieren, dass diese nahezu gut erforscht sind (bspw. Stegmann \& Kraft 1988; Schindler 1997; Schröder-Gronostay 1999 sowie diverse DZHW-Studien seit den 1970er Jahren). Auch die Frage nach der Prävention von Studienabbrüchen wird vermehrt aus der Hochschulforschung thematisiert (bspw. Pohlenz, Tinsner \& Seyfried 2012).

Die Folgen und Wirkungen von Studienabbrüchen sind sowohl bildungsökonomisch als auch bildungssystemisch relevant: Aus bildungsökonomischer Perspektive stellen Studienabbrüche aufgrund der Effizienz- bzw. Effektivitätslogik eine Verschwendung von Ressourcen bzw. eine „staatliche Fehlinvestition" (Schröder \& Daniel 1998, 13) dar, die es zu vermeiden gilt. Aus einer bildungssystemischen Sichtweise lässt sich zwischen den Bildungsteilsystemen eine mangelnde „Durchlässigkeit nach unten“ (Jahn \& Birckner 2014,14 ) bescheinigen. Im Studium erworbene Kompetenzen sind weder zertifiziert, noch am Arbeitsmarkt verwertbar (ebd.).

Die systemischen Unzulänglichkeiten beim Übergang von der Hochschule in den Ausbildungs- und Arbeitsmarkt schränken Mobilität nicht nur auf struktureller Ebene ein. Gleichzeitig können auf personaler Ebene negative Auswirkungen für die Studienabbrecher*innen vermutet werden (Bergmann 2020). 
Vor allem die auf Effizienz und Effektivität ausgelegte bildungsökonomische Perspektive evoziert, dass das vorzeitige Verlassen der Hochschule ein „Scheitern“, ein „Versagen“ bzw. „verschwendete Zeit“" darstellt. Das wiederum lässt die Annahme zu, dass Studienabbrüche auch aus der Sichtweise des Individuums als „Ausdruck eines individuellen biographischen Versagens“ (Heublein \& Wolter 2011, 214) gelten. Innerhalb des „Studienschicksals“ (Gold 1988, 191) kommt es zu „krisenhaften Situationen“ (Schindler 1997, 6), die mit einem individuellen Scheitern gleichgesetzt werden. Demgegenüber können Studienabbrüche aus individueller Sicht allerdings auch als „biographische Neuorientierung“" (Ahles et al. 2016, 130) bzw. als Korrektur- bzw. Optimierungsmöglichkeit individueller Bildungswege betrachtet werden (Meyer et al. 1999). Wenngleich bekannt ist, dass $43 \%$ der Studienabbrecher*innen nach dem Ausstieg aus dem Studium eine Berufsausbildung aufnehmen bzw. 31\% eine Berufstätigkeit ausüben (Heublein et al. 2017), ist hinsichtlich der individuellen, subjektiven beruflichen Entwicklung ein erhebliches Forschungsdesiderat festzustellen. Vor dem Hintergrund der Diskussion um Beruf und Beruflichkeit ist zu klären, welche Rolle das vorzeitige Verlassen der Hochschulen für die Individuen innerhalb ihrer Berufsbiografien einnimmt und inwieweit es Studienabbrecher*innen gelingen kann, ihren beruflichen Weg so zu gestalten, dass sich dieser als konsistenter Entwicklungsprozess in ihre Berufsbiografie einfügt.

\section{Berufliche Identitätsentwicklung}

Nach dem Studium stehen Studienabbrecher*innen vor der Herausforderung, ihren beruflichen Weg neu- bzw. umzugestalten. Unumstritten ist, dass berufsbiografisch relevante Ereignisse und Statuspassagen, wie bspw. die Berufsbzw. Studienwahl (z. B. Mischler 2017; Eberhard, Scholz \& Ulrich 2009) oder das vorzeitige Beenden des Studiums, „Formen der Orientierungen am Beruf erkennen lassen“ (Schaeper, Kühn \& Witzel 2000). Der Beruf stellt ein mehrschichtiges, fluides und (vor dem Hintergrund des Employability-Ansatzes) umstrittenes Konstrukt dar (u. a. Kraus 2007). Trotzdem ist „Beruf“ als Abstraktion für die biografische Orientierung von Studienabbrecher*innen subjektiv bedeutsam.

Für die weitere berufliche Entwicklung der Studienabbrecher*innen stellt sich die Frage, welche Auswirkungen Studienabbrüche - als objektiv diskontinuierliche Entwicklung im Lebenslauf - auf die Berufsbiografien der Individuen haben. Hinsichtlich der Subjektseite des Berufs geht es um den subjektiven Bedeutungsgehalt, was sich wiederum in vorhandenen Interessen, Neigun- 
gen sowie einer beruflichen Identitätsbildung abzeichnet. Versteht man berufliche Identität als eine ,mittels eines Lernprozesses vom Subjekt selbst herzustellende Passung zwischen Subjektivem und Objektivem (dem Beruf)“ (Unger 2008, 42), ergibt sich in innerhalb der berufsbiografischen Betrachtung der Studienabbrecher*innen die Frage, was der „Sinnhorizont des Berufs“ (ebd.) ist. So besteht einerseits die Möglichkeit, dass der Beruf innerhalb der Biografie der Studienabbrecher*innen ein sinn- und identitätsstützendes bzw. -hervorbringendes Konstrukt darstellt, der (auch losgelöst von einer formalen Qualifizierung) als Orientierungsrahmen fungiert. Andererseits ist es auch möglich, dass die Biografie primär einen Erwerbscharakter (im Sinne des Employability-Ansatzes) aufweist, in dem berufliche Identifikationsprozesse eine eher untergeordnete Rolle spielen (Bergmann 2020).

Hieraus geht die Frage hervor, inwieweit sich beide polarisierenden Blickwinkel unterstützen können bzw. inwieweit „Beruflichkeit, als dem Beruf zugrundeliegende Form, eine Brücke oder Klammer“ darstellen kann, „mit der auf individueller Ebene Diskontinuitäten in Erwerbsverlauf“ (Tiemann 2012, 50) integriert werden können. Auch wenn sich Berufs- und Erwerbsbiografien aufgrund ihrer disziplinären Verortung nicht nur terminologisch, sondern auch inhaltlich voneinander unterscheiden, erscheint es sinnvoll, beide nicht als konkurrierende biografische Ausprägungen zu betrachten. Vielmehr ist es ratsam, ein zeitadäquates Berufsverständnis als Verknüpfung zu nutzen, um (vermeintlich) diskontinuierliche biografische Verläufe zu legitimieren. Es kann bspw. sein, dass ein von Diskontinuitäten beruflicher Lebenslauf von Studienabbrecher*innen objektiv eine Erwerbsbiografie darstellt, das Individuum den biografischen Verlauf allerdings als kohärente sowie konsistente Berufsbiografie rahmt (Bergmann 2020). Da sich der individuelle Lebenslauf aufgrund der von Dynamisierung und Flexibilisierung geprägten Arbeitswelt nicht am Konzept des stetigen Lebensberufes ausrichtet, sondern von Wechseln und Brüchen geprägt ist, ergibt sich daraus folgend die Frage, worin das Subjekt seinen beruflichen Sinn konstituieren kann. Innerhalb des Austarierens zwischen der berufsbildungstheoretischen und der ökonomischen Fundierung der Objektseite des Berufs erscheint es offen, inwieweit es Individuen trotz objektiver Diskontinuitäten (hier: Studienabbrüche) gelingen kann, einen individuellen Grad an Kontinuität sowie Konsistenz innerhalb der (individualisierten) Biografie zu erzeugen und eine berufliche Identität herzustellen. Berufliche Identitätsbildung stellt für das Subjekt einen Entwicklungsprozess dar (Haußer 1983; Marcia 1966), in welchem es aufgrund von Krisen immer wieder gezwungen ist, Entscheidungen zu treffen bzw. gegebene Handlungssituationen zu hinterfragen. Berufliche Identitätsentwicklung ist dabei ein kontinuierlicher Austarierungsprozess, bei dem es für das Subjekt um das Bewusstsein von Konsistenz, Individualität und Kontinuität geht (Hoff 1990). Für diesen 
Entwicklungsprozess sind Krisen essenziell (Erikson 1963, 1989; Marcia 1966). Diese müssen nicht zwingend negativ konnotiert bzw. identitätsbedrohend sein. Vielmehr geht es innerhalb der beruflichen Identitätsentwicklung darum, bestimmte berufliche Situationen kritisch zu reflektieren, um - sofern möglic - Entscheidungen hinsichtlich der beruflichen Entwicklung (z. B. Abbruch oder Verbleib, Arbeit oder Ausbildung) zu treffen.

\section{Untersuchungsdesign}

\subsection{Entfaltung der Forschungsfrage}

Auf Grundlage der Ausführungen zum Studienabbruch einerseits und zur beruflichen Identität andererseits lässt sich die Fragestellung der vorliegenden Untersuchung konkretisieren. Da die personale Mobilitätsebene in Bezug auf den Studienabbruch innerhalb der Forschungslandschaft bis dato kaum betrachtet wurde, erhebt die vorliegende Studie den Anspruch, eine subjektorientierte Perspektive einzunehmen und die berufliche Entwicklung von ehemaligen Studierenden zu erfassen. ${ }^{1}$

Um nachvollziehen zu können, wie sich die (objektiv diskontinuierliche) berufliche Entwicklung von Studienabbrecher*innen vollzieht bzw. welche subjektiven Sichtweisen sowie Deutungs- und Orientierungsmuster damit einhergehen, richtet die vorliegende Arbeit den Blick auf deren Berufsbiografien. Folglich ergibt sich für die vorliegende Studie die nachstehende Fragestellung, welche beruflichen Identitätsentwicklungen innerhalb der berufsbiografischen Rekonstruktionen von Studienabbrecher*innen vorliegen.

\subsection{Datenerhebung, Feldzugang und Sampling}

Die beruflichen Verläufe von Studienabbrecher*innen, welche in der vorliegenden Studie das Datenmaterial darstellen, wurden mit Hilfe von autobiografisch-narrativen Interviews nach Schütze $(1981,1983)$ erhoben. Das autobiografisch-narrative Interview erzeugt Erzählungen eigens erlebter Erfahrungen, welche sowohl das faktische Handeln als auch die Erfahrungsrekapitulation des Subjekts darstellen (Schütze 1977). Insgesamt wurden 18 Interviews mit ehemaligen Studierenden erhoben, deren Auswahl auf Basis des Theoretical

1 Die Darstellung der Datenauswertung und die ausführliche Explikation der Ergebnisse sind innerhalb der (an der Otto-von-Guericke-Universität Magdeburg entstandenen) Dissertation „Verwirklicht, entwickelt, diffus. Eine biografische Analyse der beruflichen Entwicklung von StudienabbrecherInnen“ zu entnehmen (Bergmann 2020). 
Samplings erfolgte. Voraussetzungen waren, dass die Studienaussteiger*innen a) die Hochschule vor mindestens fünf Jahren ohne Abschluss verlassen und b) vor dem Studium keinen berufsqualifizierenden Abschluss erworben hatten. Die Studienabbrecher*innen hatten innerhalb des autobiografisch-narrativen Interviews die Möglichkeit, ihre individuelle berufliche Laufbahn (beispielsweise in Form einer Berufsausbildung sowie einer anschließenden Tätigkeit) sowie die damit verbundene Erfahrungsaufschichtung in der Retrospektive zu rekonstruieren. Die nachfolgende Tabelle veranschaulicht das Sample der Untersuchung, welches aufgrund des schwierigen Zugangs insgesamt als heterogen einzuschätzen ist.

Abb. 1: Sampling der Untersuchung

\begin{tabular}{|c|c|c|c|c|c|}
\hline & $\begin{array}{l}\mathrm{Al}- \\
\text { ter }\end{array}$ & $\begin{array}{l}\text { Semes- } \\
\text { ter }\end{array}$ & $\begin{array}{l}\text { Studien- } \\
\text { richtung }\end{array}$ & $\begin{array}{l}\text { Berufsausbil- } \\
\text { dung nach } \\
\text { Studium }\end{array}$ & $\begin{array}{l}\text { Tätigkeit zum } \\
\text { Interview- } \\
\text { zeitpunkt }\end{array}$ \\
\hline Paul & 31 & 7 & $\begin{array}{l}\text { Lehramt } \\
\text { Wirt./Eng. }\end{array}$ & Physiotherapeut & $\begin{array}{l}\text { Physiotherapeut } \\
\text { und Praxislehrer }\end{array}$ \\
\hline Amy & 32 & 16 & $\begin{array}{l}\text { Lehramt } \\
\text { Eng./Deu. }\end{array}$ & keine & Künstlerin \\
\hline Maria & 32 & 17 & $\begin{array}{l}\text { Soziologie/ } \\
\text { Politik }\end{array}$ & keine & Yoga-Lehrerin \\
\hline Bob & 29 & 2 & $\begin{array}{l}\text { Südostasienkd. } \\
\text { / Informatik }\end{array}$ & $\begin{array}{l}\text { Fachinforma- } \\
\text { tiker }\end{array}$ & IT-Manager \\
\hline Walter & 34 & 11 & $\begin{array}{l}\text { Jura/Rechts- } \\
\text { wiss. }\end{array}$ & keine & $\begin{array}{l}\text { Leiter } \\
\text { Möbelbranche }\end{array}$ \\
\hline Sven & 40 & 10 & Jura & keine & Leiter Einkauf \\
\hline Mark & 33 & 13 & $\begin{array}{l}\text { Soziologie/ } \\
\text { Politik }\end{array}$ & $\begin{array}{l}\text { Konstruktions- } \\
\text { mechaniker }\end{array}$ & $\begin{array}{l}\text { Konstruktions- } \\
\text { mechaniker }\end{array}$ \\
\hline Lilly & 27 & 2 & $\begin{array}{l}\text { Kunstgesch./ } \\
\text { Musikwiss. }\end{array}$ & Fotografin & Fotografin \\
\hline Clemens & 30 & 6 & $\begin{array}{l}\text { Wirt.ingenieur } \\
\text {-wesen }\end{array}$ & $\begin{array}{l}\text { Kaufmann im } \\
\text { Einzelhandel }\end{array}$ & $\begin{array}{l}\text { Leiter } \\
\text { Einzelhandel }\end{array}$ \\
\hline Sabrina & 34 & 13 & $\begin{array}{l}\text { Soziologie/ } \\
\text { Pädagogik; } \\
\text { Lehramt } \\
\text { Wirt./Eng. }\end{array}$ & $\begin{array}{l}\text { Kauffrau im } \\
\text { Einzelhandel }\end{array}$ & $\begin{array}{l}\text { Handelsfach- } \\
\text { wirtin } \\
\text { Einzelhandel }\end{array}$ \\
\hline
\end{tabular}




\begin{tabular}{|c|c|c|c|c|c|}
\hline Robert & 24 & 1 & Maschinenbau & $\begin{array}{l}\text { Industrie- } \\
\text { kaufmann }\end{array}$ & $\begin{array}{l}\text { Industrie- } \\
\text { kaufmann }\end{array}$ \\
\hline Brad & 32 & 9 & Sport/Technik & $\begin{array}{l}\text { Kaufmann im } \\
\text { Einzelhandel }\end{array}$ & $\begin{array}{l}\text { Einzelhandels- } \\
\text { kaufmann }\end{array}$ \\
\hline Fiona & 26 & 4 & $\begin{array}{l}\text { Öffentliche } \\
\text { Verwaltung }\end{array}$ & $\begin{array}{l}\text { Kauffrau im } \\
\text { Einzelhandel }\end{array}$ & $\begin{array}{l}\text { Vertrieb } \\
\text { Großhandel }\end{array}$ \\
\hline Simon & 35 & 8 & $\begin{array}{l}\text { Wirt.ingenieur } \\
\text {-wesen/ Wirt.- } \\
\text { informatik }\end{array}$ & Hotelfachmann & Informatiker \\
\hline Mick & 37 & 13 & $\begin{array}{l}\text { BWL/ } \\
\text { Erw.bildung }\end{array}$ & $\begin{array}{l}\text { Kaufmann } \\
\text { Groß- u. } \\
\text { Außenhandel }\end{array}$ & IT-Manager \\
\hline Steffen & 35 & 8 & Elektrotechnik & $\begin{array}{l}\text { Verfahrens- } \\
\text { mechaniker }\end{array}$ & $\begin{array}{l}\text { Verfahrens- } \\
\text { mechaniker }\end{array}$ \\
\hline Susi & 30 & 5 & $\begin{array}{l}\text { Sicherheits- u. } \\
\text { Gefahrenab- } \\
\text { wehr }\end{array}$ & $\begin{array}{l}\text { Kauffrau im } \\
\text { Groß- und } \\
\text { Einzelhandel }\end{array}$ & $\begin{array}{l}\text { Vertrieb } \\
\text { Großhandel }\end{array}$ \\
\hline
\end{tabular}

Quelle: Bergmann 2020, 127

Die Heterogenität zeigt sich in Bezug auf Alter, Studiendauer (in Semestern), Fachrichtung, beruflicher Qualifizierung nach dem Studium sowie der aktuellen Tätigkeit. Anhand der beiden letzten Aspekte wird deutlich, dass unterschiedliche Formen des Übergangs in die Arbeitswelt erfasst werden. Darüber hinaus sind alle Probanden*innen zum Zeitpunkt der Interviews in den Arbeitsmarkt integriert.

\subsection{Datenauswertung}

Die Datenauswertung erfolgte anhand der Systematik der Grounded Theory (Strauss \& Corbin 2010; Tiefel 2005) sowie der biografieanalytischen Methode (Schütze 1981, 1983). Im zirkulären Auswertungsprozess wurde das von Tiefel (2005) auf erziehungswissenschaftliche Biografieforschung angepasste Kodierparadigma genutzt. Dadurch können nicht nur die Sinnkonstruktionen der Subjekte (also der Studienabbrecher*innen), sondern auch die die Biografie umgebenden Strukturen bzw. sozialen Rahmen herausgearbeitet werden, um letztendlich auf die konkreten Handlungsaktivitäten zu schließen (Tiefel 2005). Im Zuge des zirkulären Auswertungsprozesses (ausführlich Bergmann 2020) wurden drei Gruppen aus dem Material heraus extrahiert. Innerhalb der 
gebildeten Gruppen werden die zugeordneten Fälle hinsichtlich ihrer internen Homogenität verglichen (,Ebene des Typus“). Zudem erfolgt auf der „Ebene der Typologie" ein Vergleich der Gruppen untereinander. Es geht also um das Herausstellen einer genügend hohen externen Heterogenität, d. h. um die Varianz der herausgebildeten Typen sowie um eine Analyse inhaltlicher Sinnzusammenhänge (Kelle \& Kluge 2010). Insgesamt lassen sich anhand der biografieanalytischen Untersuchung drei verschiedene Typen beruflicher Identitätsentwicklung identifizieren: Identitätsverwirklicher, Identitätsdiffuse sowie Identitätsentwickler. Ihre biografischen Verläufe unterscheiden sich hinsichtlich ihrer Handlungsweisen, ihrer subjektiven Deutungen, ihrer strukturellen Bindungen sowie ihrer Prozesshaftigkeit. Die Handlungsweisen bilden vor allem die berufsbiografischen Entscheidungen und den Umgang mit Krisen innerhalb der beruflichen Entwicklung ab (Bergmann 2020). Die subjektiven Deutungen lassen sich anhand des Krisenempfindens und der Attributionsmuster sowie anhand der Ausprägung eines sinnstiftenden Identifikationsankers spezifizieren (ebd.). Für die Berufsbiografien kann sowohl die Bindung zum Beruf als auch zu sozialen Akteure strukturgebend sein (ebd.). Bei der Prozesshaftigkeit der berufsbiografischen Darstellungen geht es um die Frage, wie die Individuen innerhalb ihrer rekonstruierten Biografie den Prozesscharakter, also die Verkettung von Einstiegen, Übergängen bzw. Wechseln sowie die Prozesslogik bzw. -dynamik darstellen (ebd.).

\section{Typen beruflicher Identitätsentwicklung}

\section{Typus 1: „Identitätsverwirklicher“ (Simon, Bob, Lilly, Sven, Max, Mick)}

Handlungsweisen: Identitätsverwirklicher zeichnen sich innerhalb der beruflichen Entwicklung durch Entscheidungen aus, die im Spannungsfeld zwischen Selbstbestimmung, Selbstinitiative und Ereignetem getroffen werden. Die Entscheidungen werden vom Subjekt aus initiiert und werden selbstbestimmt getroffen. Obwohl das Handeln dieses Typus nicht primär intendiert, sondern eher von Ereignetem bestimmt wird, ist es vom aktiven Agieren des Biografieträgers gekennzeichnet. Berufliche Optionen werden angenommen und für sich genutzt. Darüber hinaus erfolgt eine bewusste Abwägung von Alternativen. Entscheidungen werden primär auf Grundlage vorhandener Interessen und Neigungen getroffen. Treten innerhalb der beruflichen Entwicklung Krisen auf, zeichnet sich ihr Handeln durch Exploration, das heißt durch ein aktives Suchen bzw. Streben nach beruflichen Alternativen aus. Auftretende Krisen beeinflussen die Handlungslogik des Biografieträgers, indem diese durch eine proaktive Kompensation für die berufliche Gestaltung genutzt werden. 
Subjektive Deutung: Ein Charakteristikum dieses Typus ist es, dass die berufliche Entwicklung, welche bspw. mit (Studienab-)Brüchen bzw. Wechseln durchsetzt ist, in der subjektiven Wahrnehmung nicht zwingend krisenbehaftet sein muss. Wenngleich es objektiv zu einer Einschränkung der Person-Umwelt-Passung kommt, stellt dieses Mismatch keine unmittelbare identitätsbedrohende Krise dar. In Bezug auf die Attributionsmuster zeichnet sich dieser Typus durch Variabilität aus (internal/ external bzw. global/ spezifisch). Identitätsverwirklicher folgen von Beginn des universitären Bildungsweges an ihrem beruflichen Interessenszentrum. Innerhalb der gesamten beruflichen Entwicklung richten sie ihr Handeln entlang eines inneren, sinnstiftenden Identifikationsankers aus.

Strukturelle Ebene: Institutionelle berufliche Möglichkeits- und Gestaltungsräume werden antizipiert und proaktiv ausgenutzt. Der entsprechende Beruf fungiert in diesem Kontext unabhängig von einer formalen Qualifizierung als subjektiv bedeutsames Konstrukt einerseits und als strukturgebender Handlungsrahmen andererseits (Unger 2008). Aufgrund des identitätsstiftenden Charakters ist die Bindung an den Beruf sehr ausgeprägt. Dabei ist es für die subjektive Bedeutsamkeit des strukturgebenden beruflichen Rahmens irrelevant, ob eine formale Qualifizierung bspw. durch den institutionellen Rahmen der Berufsausbildung vorliegt. Sowohl formal als auch nicht-formal qualifizierte Studienabbrecher*innen weisen hinsichtlich ihres äußerlich ausgeübten Berufs eine innere Verbundenheit auf, welche ausschlaggebend für die berufliche Identifikation ist. Neben dem Beruf als orientierungs- bzw. strukturgebendes Konstrukt sind auf der strukturellen Ebene zudem soziale Beziehungen (bspw. in Form von Kommiliton*innen) relevant, welche die berufliche Entwicklung der ehemaligen Studierenden beeinflussen. Das Subjekt zeichnet sich durch ein Weltbild aus, in welchem signifikante Andere als orientierungsgebende und unterstützende soziale Faktoren agieren.

Prozessebene: Der Typus des Identitätsverwirklichers zeichnet sich über die Rekonstruktion der Berufsbiografie hinweg durch eine ,glückliche“ Verkettung von beruflichen Einstiegen, Übergängen bzw. Wechseln aus. Trotz eines objektiv diskontinuierlichen beruflichen Lebensablaufs ergeben sich für das Subjekt zufällige und glückliche Fügungen, welche es für sich nutzt, so dass die berufliche Entwicklung von einer subjektiv konsistenten, individualisierten und kontinuierlichen (rekonstruierten) Sichtweise geprägt ist.

Typus 2: „Identitätsdiffuse“ (Paul, Sabrina, Fiona, Robert, Steffen, Clemens, Mark, Walter)

Handlungsweisen: Die berufsbiografische Entscheidungsebene der Identitätsdiffusen ist primär durch berufliche Entschlüsse (und nicht Entscheidungen) gekennzeichnet, welche wiederum eine eher reaktive, alternativlose Konse- 
quenz auf Ereignetes darstellen. Auch wenn die Entscheidungen selbstbestimmt getroffen werden, stellen diese primär einen Ausweg aus der jeweiligen Situation dar, um die entsprechende Handlungsfähigkeit aufrecht zu erhalten. Der Umgang mit Krisen ist von Verteidigung bzw. Aufrechterhaltung des beruflichen Status quo geprägt. Eine aktive Exploration beruflicher Alternativen findet überwiegend nicht statt. Vielmehr sind die Handlungsweisen der Identitätsdiffusen von Passivität geprägt.

Subjektive Deutung: Dieser Typus erlebt Krisen sowohl als gering als auch als identitätsbedrohend und nimmt eine variable Ursachenzuschreibung kritischer Situationen vor. Während die Fälle, die Krisen als identitätsbedrohend ansehen, diese external und global attribuieren, zeichnen sich diejenigen mit einem geringem Krisenempfinden durch einen internalen und spezifischen Attributionsstil aus. Die berufliche Entwicklung der Identitätsdiffusen ist von einer eher diffusen Interessenlage gekennzeichnet. Innerhalb ihrer gesamten beruflichen Entwicklung gelingt es den ehemaligen Studierenden nicht, einen inneren beruflichen Sinn zu konstituieren. Vielmehr orientiert sich deren berufliche Entwicklung an instrumentellen Faktoren wie bspw. Abschlüssen oder Verdienstmöglichkeiten.

Strukturelle Bindung: Für diesen Typus ist es charakteristisch, dass die Handlungen immer in einem strukturgebenden Rahmen stattfinden. Diesem institutionellen Rahmen (bspw. das Studium oder die Berufsausbildung) kommt eine orientierungsgebende und schützende Funktion zu. Sind Identitätsdiffuse dazu angehalten, diesen Rahmen (aus differenzierten Gründen) zu verlassen, begeben sich diese in ein neues, von Sicherheit geprägtes Muster. Das eher passiv handelnde Subjekt wird dabei von äußeren Faktoren gesteuert. Aufgrund dieser Passivität gelingt es den Identitätsdiffusen nicht, sich an die entsprechende berufliche Tätigkeit subjektiv zu binden. Trotz dieser mangelnden Bindung benötigt der Typus des Identitätsdiffusen innerhalb der beruflichen Entwicklung eine institutionelle stabilisierende Struktur. Dabei ist es für das Subjekt unerheblich, ob der strukturgebende Anker der formale Beruf oder die derzeit ausgeübte Tätigkeit ist. Trotz der Suche nach einem strukturgebenden Anker haben soziale Beziehungen in der Rolle der signifikant Anderen eine eher untergeordnete Bedeutung. Der Typus der Identitätsdiffusen weist eher eine niedrige Ausprägung sozialer Bindungen vor, welche als Orientierungs- bzw. Unterstützungsfaktoren den beruflichen Weg beeinflussen.

Prozessebene: Der Typus zeichnet sich durch eine ,ereignete“ Verkettung von beruflichen Einstiegen, Übergängen bzw. Wechseln aus. Für die Fälle der Identitätsdiffusen, welche Krisen als identitätsbedrohend wahrnehmen, ist der berufliche Werdegang nicht nur auf objektiver, sondern auch auf subjektiver Ebene ein eher inkonsistenter und diskontinuierlicher Weg voller Brüche und Wechsel. Das Subjekt weist in diesem Fall ein Gefühl der Gespaltenheit, der Zerrissenheit sowie der Austauschbarkeit auf. Diejenigen Identitätsdiffusen, 
welche die Situationen innerhalb ihrer beruflichen Entwicklung als gering krisenbehaftet deuten, rekonstruieren ihre Berufsbiografie als konsistenten und kontinuierlichen Weg.

\section{Typus 3: „Identitätsentwickler“ (Amy, Brad, Maria, Susi)}

Handlungsweisen: Die Entscheidungsgestalt dieses Typus zeichnen sich durch ihren Wandel aus. Während die beruflichen Entscheidungen bzw. Entschlüsse vor bzw. innerhalb des Studiums primär von äußeren Begebenheiten (wie bspw. fehlende Zulassungsbeschränkung oder der Übernahme familiär tradierter Erwartungen) sowie von Reaktivität geprägt waren und ein bewusstes Abwägen interessengeleiteter Alternativen eher zu kurz kam, gelingt dem Typus des Identitätsentwicklers aufgrund von biografischen Wendepunkten innerhalb der beruflichen Entwicklung ein Wandel zur selbstbestimmten Entscheidungsfähigkeit. Es handelt sich nicht mehr um ein Reagieren, sondern um ein bewusstes biografisches Agieren, welches von Interessen und Neigungen bestimmt wird. Während dieser Typus anfänglichen Krisen primär passiv begegnet, vollzieht sich innerhalb der beruflichen Entwicklung ein Wandel zu einem aktiven Explorationsverhalten.

Subjektive Deutung: Obwohl innerhalb des Umgangs mit Krisen ein Wandel zu verzeichnen ist, ist das entsprechende Attributionsmuster von Stabilität geprägt (internal und global). Die berufliche Entwicklung dieses Typus ist überwiegend von einem Wendepunkt gekennzeichnet, welcher als ein Auslöser eines Identitätsentwicklungsprozesses fungiert. Mit diesem biografischen Wendepunkt entwickeln die ehemaligen Studierenden sukzessive berufliche Interessen und Neigungen im Sinne einer beruflichen Sinnkonstitution und setzen diese in ihren Tätigkeiten um. Den Identitätsentwicklern gelingt es also über ihre berufliche Entwicklung hinweg, eine berufliche Identität zu entwickeln.

Strukturelle Ebene: Innerhalb des berufsbiografischen Verlaufs des Typus des Identitätsentwicklers kommt dem Beruf nach dem entsprechenden (impliziten oder expliziten) Wendepunkt die Funktion eines strukturgebenden Handlungsrahmens zu. Berufliche Möglichkeits- und Gestaltungsspielräume werden zum Zeitpunkt des Interviews vollends ausgeschöpft und fungieren als Orientierungsgrundlage. Das Subjekt und der entsprechende Beruf zeichnen sich durch eine ausgeprägte Verbundenheit aus. Dabei ist es unerheblich, ob für diesen Beruf eine formale Qualifizierung vorhanden ist. Während die Verbundenheit an die berufliche Station des Studiums bzw. hinsichtlich sozialer Akteure zu Beginn der beruflichen Entwicklung eher rudimentär war, unterliegen beide Aspekte einem Wandlungsprozess, sodass die Biografieträger zum Zeitpunkt des Interviews eine ausgeprägte berufliche bzw. soziale Verbundenheit aufweisen. 
Prozessebene: Insgesamt ist der berufliche Weg dieses Typus durch eine „erarbeitete" Verkettung von Einstiegen, Übergängen und Wechseln geprägt. Obwohl die berufliche Entwicklung noch nicht abgeschlossen ist, ist aufgrund des Wandlungsprozesses zu erwarten, dass die ehemaligen Studierenden ihren beruflichen Weg als konsistent und kontinuierlich rahmen. Der Beginn der beruflichen Entwicklung (mit dem Studium) zeichnet sich eher von Destabilisierung und Desorientierung aus und wandelt sich erst mit einem expliziten bzw. impliziten Wendepunkt zum entsprechenden Gegenpol. Dieser Wendepunkt stellt zwar objektiv einen Verkettungsbruch dar, allerdings wird die berufliche Entwicklung über den Prozess hinweg von den Subjekten als konsistent und kontinuierlich wahrgenommen.

\section{Fazit}

Aus den Ergebnissen zur beruflichen Identitätsentwicklung von Studienabbrecher*innen wird deutlich, dass es in unterschiedlicher Weise gelingen kann, die berufliche Laufbahn so zu gestalten, dass diese sich als konsistentes Entwicklungsprojekt in ihre Berufsbiografie einfügt: Einem Teil der ehemaligen Studierenden gelingt es, ihre berufliche Identität entlang eines beruflichen Identifikationsankers zu konstituieren (Identitätsverwirklicher) bzw. kontinuierlich zu entwickeln (Identitätsentwickler). Demgegenüber lassen sich berufliche Verläufe verzeichnen, deren berufliche Identitätsentwicklung von Diffusion und einer Orientierung an äußeren Faktoren (bspw. Verdienstmöglichkeiten) geprägt sind (Identitätsdiffuse). Unabhängig von der Ausprägung der beruflichen Identitätsentwicklung wird allerdings deutlich, dass die Subjekte als Gestalter und Ausrichter der eigenen Berufsbiografie im Mittelpunkt stehen. Sie sind innerhalb ihrer (objektiv diskontinuierlichen) beruflichen Verläufe permanent gefragt, berufliche Möglichkeiten auszuschöpfen und Orientierungsleistungen $\mathrm{zu}$ erbringen, um subjektiv anschlussfähig zu sein. Es wird also deutlich, dass die Berufsbiografien der Studienabbrecher*innen personale Mobilität aufzeigen. Auch wenn dieser berufliche Weg als subjektives und von Krisen durchsetztes Scheitern bilanziert wird, zeigt sich anhand der Biografieanalysen dennoch, dass es vielmehr um einen identitätsentwickelnden (Bildungs-)Prozess geht, in welchem sich das Subjekt auf eine berufliche Platzsuche begibt. Unabhängig von der Konnotation des eigenen beruflichen Verlaufs wird deutlich, dass personale (berufliche) Mobilität bzw. die subjektiven beruflichen Orientierungsleistungen eine intensive Auseinandersetzung mit der eigenen beruflichen Identität erfordert. 


\section{Literatur}

Ahles, L., Köstler, U., Vetter, N. \& Wulff, A. (2016). Studienabbrüche an deutschen Hochschulen. Stand der Thematisierung und strategische Ansatzpunkte. BadenBaden: Nomos (Studien zum sozialen Dasein der Person).

Bergmann, D. (2016). Studien- und Ausbildungsabbrüche im Vergleich. Berufsbildung: Zeitschrift für Theorie, Praxis, Dialog, 70(157), 22-24.

Bergmann, D. (2020). Verwirklicht, entwickelt, diffus. Eine biografische Analyse der beruflichen Entwicklung von StudienabbrecherInnen. Wiesbaden: Springer.

Dick, M. (2009). Mobilität als Tätigkeit. Individuelle Expansion - alltägliche Logistik kulturelle Kapazität. Lengerich: Pabst.

Eberhard, V., Scholz, S. \& Ulrich, J. G. (2009). Image als Berufswahlkriterium. Bedeutung für Berufe mit Nachwuchsmangel. Berufsbildung in Wissenschaft und Praxis, 38(3), 9-13. Online: www.bibb.de/veroeffentlichungen/de/bwp/show/1584 (06.04.2020).

Erikson, E. H. (1989). Identität und Lebenszyklus. Drei Aufsätze (11. Aufl.). Frankfurt a. M.: Suhrkamp.

Frommberger, D. (2019). Berufliche und hochschulische Bildung im Wandel - Entwicklungen zwischen Annäherung, Differenzierung und Öffnung. In B. Hemkes \& K. Wilbers (Hrsg.), Durchlässigkeit zwischen beruflicher und hochschulischer Bildung (S. 36-59). Bielefeld: Bertelsmann Verlag.

Gold, A. (1988). Studienabbruch, Abbruchneigung und Studienerfolg. Vergleichende Bedingungsanalysen des Studienverlaufs. Frankfurt a. M. und Bern: P. Lang.

Haußer, K. (1983). Identitätsentwicklung. New York: Harper \& Row.

Heublein, U. \& Wolter, A. (2011). Studienabbruch in Deutschland. Zeitschrift für Pädagogik, 2, 214-235.

Heublein, U., Ebert, J., Hutzsch, C., Isleib, S., König, R., Richter, J. \& Woisch, A. (2017). Zwischen Studienerwartungen und Studienwirklichkeit. Ursachen des Studienabbruchs, beruflicher Verbleib der StudienabbrecherInnen und Studienabbrecher und Entwicklung der Studienabbruchquote an deutschen Hochschulen. Hannover: DZHW.

Heublein, U., Hutzsch, C., König, R., Kracke, N. \& Schneider, C. (2018). Die Attraktivität der beruflichen Bildung bei Studienabbrecherinnen und Studienabbrechern. (Reihe Berufsbildungsforschung, Band 18). Berlin: BMBF.

Hemkes, B. \& Wilbers, K. (2019). Einführung: Herausforderung Durchlässigkeit - Versuch einer Näherung. In B. Hemkes, K. Wilbers \& Michael Heister (Hrsg.), Durchlässigkeit zwischen beruflicher und hochschulischer Bildung (S. 1-33). Leverkusen: Budrich.

Hoff, E.-H. (1990). Identität und Arbeit. In C. Baitsch \& E. Ulich (Hrsg.), Arbeit und Identität (S. 7 -25). München: Psychologie Verlag.

Jahn, R. W. \& Birckner, M. (2014). Studienabbrecher. Über die Situation einer (noch) kaum beachteten Zielgruppe innerhalb und außerhalb der Beruflichen Bildung. Jena: JIBB.

Kelle, U. \& Kluge, S. (2010). Vom Einzelfall zum Typus. Fallvergleich und Fallkontrastierung in der qualitativen Sozialforschung (2. überarb. Aufl.). Wiesbaden: VS Verlag für Sozialwissenschaften. 
Kraus, K. (2007). Vom Beruf zur Employability? Zur Theorie einer Pädagogik des Erwerbs. Wiesbaden: VS Verlag für Sozialwissenschaften.

Marcia, J. E. (1966). Development of a validation of ego identity status. Journal of Personality and Social Psychology, 3(5), 551-558.

Meyer, T., Diem, M., Droz, R., Galley, F. \& Kiener, U. (1999). Hochschule - StudiumStudienabbruch. Synthesebericht zum Forschungsprojekt "Studienabbruch an schweizerischen Hochschulen als Spiegel von Funktionslogiken ". Chur: Rüegger.

Mischler, T. (2017). Die Attraktivität von Ausbildungsberufen im Handwerk. Eine empirische Studie zur beruflichen Orientierung von Jugendlichen. Bielefeld: W. Bertelsmann Verlag. Online: https://www.bibb.de/veroeffentlichungen/ de/publication/show/8551 (06.04.2020).

Pohlenz, P., Tinsner, K. \& Seyfried, M. (2012). Studienabbruch. Ursachen, Probleme, Begründungen. Saarbrücken: AV Akademikerverlag.

Schaeper, H., Kühn, Th. \& Witzel, A. (2000). Diskontinuierliche Erwerbskarrieren und Berufswechsel in den 1990ern. Strukturmuster und biografische Umgangsweisen betrieblich ausgebildeter Fachkräfte. Mitteilungen aus der Arbeitsmarkt- und Berufsforschung, 33(1), 80-100.

Schindler, G. (1997). "Frühe" und "späte" Studienabbrecher. München: Bayerisches Staatsinstitut für Hochschulforschung und Hochschulplanung.

Schröder, M. \& Daniel, H. D. (1998). Studienabbruch. Eine annotierte Bibliographie (1975-1997). Kassel: Jenior und Pressler.

Schröder-Gronostay, M. (1999). Studienabbruch - Zusammenfassung des Forschungsstandes. In M. Schröder-Gronostay \& H. D. Daniel (Hrsg.), Studienerfolg und Studienabbruch. Beiträge aus Forschung und Praxis (S. 209-240). Neuwied: Luchterhand.

Schütze, F. (1977). Die Technik des narrativen Interviews in Interaktionsfeldstudien. Dargestellt an einem Projekt zur Erforschung von kommunalen Machtstrukturen. Bielefeld: Arbeitsberichte und Forschungsmaterialien/ Universität Bielefeld, Fakultät für Soziologie.

Schütze, F. (1981). Prozessstrukturen des Lebenslaufes. In Biographie in handlungswissenschaftlicher Perspektive (S. 67-156), Kolloquium am Sozialwissenschaftlichen Forschungszentrum der Universität Erlangen-Nürnberg. Nürnberg: Verl. d. Nürnberger Forschungsvereinigung.

Schütze, F. (1983). Biographieforschung und narratives Interview. Neue Praxis, 13, 283-293.

Stegmann, H. \& Kraft, H. (1988). Bestimmungsfaktoren für den Studienabbruch und Berufswege von Studienabbrechern. Mitteilungen aus der Arbeitsmarkt- und Berufsforschung, 21(4), 498-511.

Strauss, A. L.\& Corbin, J. M. (2010). Grounded Theory. Grundlagen qualitativer Sozialforschung (unveränd. Nachdr. der letzten Aufl.). Weinheim: Beltz.

Tiefel, S. (2005). Kodierung nach der Grounded Theory lern- und bildungstheoretisch modifiziert. Zeitschrift für qualitative Bildungs-, Beratungs- und Sozialforschung, $6(2), 65-84$.

Tiemann, M. (2012). Die Entwicklung von Beruflichkeit im Wandel der Arbeitswelt. In A. Bolder, R. Dobischat, G. Kutscha \& G. Reutter (Hrsg.), Beruflichkeit zwischen institutionellem Wandel und biographischem Projekt (S. 49-72). Wiesbaden: VS Verlag für Sozialwissenschaften. 
Unger, T. (2008). Wissensstrukturen beruflicher Identität. Ausgewählte theoretische Grundlagen einer Längsschnittstudie zur Entwicklung beruflicher Identität in einem Leistungspunktesystem in der beruflichen Bildung. In BMBF (Hrsg.), Entwicklung eines Leistungspunktesystems in der beruflichen Bildung (S. 41-52).

Wissenschaftsrat (2014). Empfehlungen zur Gestaltung des Verhältnisses von beruflicher und akademischer Bildung - Erster Teil der Empfehlungen zur Qualifizierung von Fachkräften vor dem Hintergrund des demographischen Wandels. Darmstadt. Online:

https://www.wissenschaftsrat.de/download/archiv/3818-14.pdf (06.04. 2020). 



\section{Vorzeitige Vertragslösungen aus der Perspektive der Auszubildenden - ein Vorschlag für einen subjektorientierten Erklärungsansatz}

Silke Lange

\section{Einleitung}

Vorzeitige Vertragslösungen und Ausbildungsabbrüche zählen aufgrund der seit den 1980er Jahren gestiegenen Vertragslösequoten bundesweit zu den zentralen Problemfeldern der dualen Berufsausbildung. Erklärt werden vorzeitige Vertragslösungen im wissenschaftlichen Diskurs über verschiedene Ansätze. Empirisch zeigt sich, dass ein Ansatz allein nicht ausreicht, sondern das Vertragslösegeschehen komplex und die Ursachen vielfältig und mehrdimensional sind. Das deutet darauf hin, dass vorliegende Erklärungsansätze das Vertragslösegeschehen nicht ausreichend modellieren können, sondern nur einzelne Bereiche und Einflussfaktoren des Gegenstands erfassen. Das Vertragslösegeschehen ist folglich als ,prozesshaftes Geschehen zu betrachten [...], das nicht eine Ursache hat, sondern von mehreren Faktoren bestimmt wird" (Jasper et al. 2009, 12). Klaus ergänzt die vorliegenden Ansätze daher um ein „Prozessmodell“ (2014, 225ff.), das die Identitäts- und Persönlichkeitsentwicklung der Betroffenen fokussiert. Das Vertragslösegeschehen wird in diesem Modell als mehrphasiger Prozess beschrieben, der durch unterschiedliche Schlüsselereignisse strukturiert ist und durch verschiedene Auslöser und Gründe initiiert und verstärkt wird. Damit legt er einen Ansatz vor, der die subjektive Perspektive der betroffenen Auszubildenden auf die vorzeitige Vertragslösung hervorhebt und den Prozess aus dieser Perspektive analysiert und erklärt. Folglich ist ,nicht der Prozess selbst individuell, sondern nur die Kombination aus Gründen und Motiven“" (ebd., 420).

Aufbauend auf eine Untersuchung zur Berufsausbildungseingangsphase soll im Beitrag ein Vorschlag für einen ergänzenden subjektorientierten Erklärungsansatz geliefert werden, der die wahrgenommene fehlende Passung auf Defizite individueller Bedürfnisse der Jugendlichen zurückführt und damit einen Strukturierungsvorschlag für die individuelle Kombination aus Gründen und Motiven liefert. Im Fokus des Beitrages steht daher die Fragestellung, wie die individuelle Kombination von Gründen und Motiven vorzeitiger Vertragslösungen erklärt und strukturiert werden kann? 
Im folgenden zweiten Abschnitt wird zunächst der Problemzusammenhang der vorzeitigen Vertragslösungen in der dualen Berufsausbildung weiter ausgeführt. Auf Basis der im dritten Kapitel vorgestellten Untersuchung zur Berufsausbildungseingangsphase wird im vierten Kapitel ein Ansatz zur Erklärung der Gründe und Motive vorzeitiger Vertragslösungen aus subjektiver Perspektive der Auszubildenden entworfen, der einen theoretischen Beitrag zum berufs- und wirtschaftspädagogischen Diskurs vorzeitiger Vertragslösungen leistet.

\section{Vorzeitige Vertragslösungen}

Die duale Berufsausbildung nimmt in Deutschland eine zentrale Funktion in der Entwicklung einer Vielzahl von Jugendlichen und jungen Erwachsenen und ihrer beruflichen Handlungskompetenz ein. Zu den zentralen Problemfeldern der dualen Berufsausbildung zählen vorzeitige Vertragslösungen, die für die Jugendlichen nicht selten soziale und psychische Konsequenzen haben. Doch nicht nur für die Jugendlichen, sondern auch für Ausbildungsbetriebe sind Vertragslösungen mit Unsicherheiten und einem Verlust von Zeit und Ressourcen verbunden (BMBF 2019). Daher zählt die Vermeidung vorzeitiger Vertragslösungen bis heute zu den berufsbildungspolitischen Zielsetzungen (Allianz für Aus- und Weiterbildung 2019) und wird mit einer Reihe von Aktivitäten umgesetzt (ebd.).

Unter einer vorzeitigen Vertragslösung wird die Aufkündigung eines angetretenen Ausbildungsvertrages vor dem vertraglich vereinbarten Ausbildungsende von einer der beiden Seiten (Auszubildende oder Betriebe) oder von beiden Seiten verstanden. Die Ausbildungsverhältnisse unterliegen dabei den besonderen Kündigungsschutzbestimmungen des Berufsbildungsgesetzes (§ 22). Demnach können Ausbildungsverhältnisse innerhalb der Probezeit von beiden Seiten jederzeit gekündigt werden. Außerhalb der Probezeit kann eine Kündigung nur noch im gegenseitigen Einvernehmen oder aus wichtigem Grund vonseiten des Ausbildungsbetriebes und vonseiten des Auszubildenden jederzeit unter Einhaltung einer vierwöchigen Kündigungsfrist erfolgen.

Etwa jeder achte Auszubildende eines Ausbildungsjahrgangs macht von seinem Kündigungsrecht Gebrauch und löst den Ausbildungsvertrag vorzeitig (u. a. Piening et al. 2010). Dazu kommen die Ausbildungsverhältnisse, die vonseiten der Ausbildungsbetriebe oder im gegenseitigen Einvernehmen gelöst werden. Insgesamt wird derzeit etwa ein Viertel der Ausbildungsverträge eines Ausbildungsjahrgangs vorzeitig gelöst, das zeigt die regelmäßige Berufsbildungsberichterstattung (BIBB 2017; Lange 2019). Die darin jährlich ausge- 
wiesene Lösungsquote ${ }^{1}$ beschreibt näherungsweise den Anteil der neu abgeschlossenen Ausbildungsverträge eines Ausbildungsjahrgangs, die vorzeitig gelöst werden.

Seit den 1990er Jahren schwanken die Lösungsquoten zwischen $20 \%$ und $25 \%$, wobei die Lösungsquoten in den letzten Jahren leicht überhalb des üblichen Schwankungsbereichs lagen (BMBF 2019). Dabei zeigen sich deutliche Unterschiede zwischen unterschiedlichen Ausbildungsberufen und Berufsbereichen; von vorzeitigen Vertragslösungen besonders betroffen ist das Handwerk (BMBF 2019; Lange 2019). Darüber hinaus gelten verschiedene sozioökonomische Merkmale der Auszubildenden (u. a. Geschlecht, Staatsbürgerschaft, Schulabschluss) sowie markt- und ausbildungsbetriebsspezifische Merkmale (u. a. Ausbildungsbetriebsgröße, Region, Ausbildungsmodell) als Risikofaktoren vorzeitiger Vertragslösungen (zusammenfassend Lange 2019).

Auch innerhalb des Ausbildungsverlaufs unterscheidet sich das Risiko vorzeitiger Vertragslösungen. Etwa ein Drittel der Vertragslösungen erfolgt innerhalb der Probezeit, ein weiteres Drittel der vorzeitig gelösten Verträge wird nach der Probezeit im ersten Ausbildungsjahr gelöst. Anschließend sinkt das Risiko vorzeitiger Vertragslösungen deutlich; im zweiten Ausbildungsjahr erfolgt etwa ein Viertel der Vertragslösungen, anschließend liegt der Anteil der Vertragslösungen (an allen Vertragslösungen) bei knapp 10\%. Der Anteil der frühen vorzeitigen Vertragslösungen (im ersten Ausbildungsjahr) ist seit 1993 von knapp der Hälfte der Vertragslösungen auf zwei Drittel aller Vertragslösungen gestiegen, während der Anteil der Vertragslösungen im zweiten Ausbildungsjahr kontinuierlich gesunken ist ${ }^{2}$ (Lange 2019, 66). Zurückzuführen ist der Anstieg der frühen vorzeitigen Vertragslösungen auf einen Anstieg der Vertragslösequote sowohl während der Probezeit (von rund $25 \%$ auf rund $35 \%$ ) als auch innerhalb des ersten Ausbildungsjahres nach der Probezeit (von rund $24 \%$ auf rund $31 \%$ ) (ebd.). Das erste Jahr der Berufsausbildung weist folglich ein besonderes Potential vorzeitiger Vertragslösungen auf.

Zur Erklärung vorzeitiger Vertragslösungen wird auf verschiedene Ansätze zurückgegriffen, die das Phänomen aus unterschiedlichen Perspektiven und Blickwinkeln analysieren und interpretieren:

- Aus einer ökonomischen Perspektive werden vorzeitige Vertragslösungen als Folge struktureller und konjunktureller Faktoren der regionalen Wirtschaftsstruktur erklärt (Weiss 1982). Rohrbach-Schmidt und Uhly

1 Berechnet wird die Lösungsquote seit dem Berichtsjahr 2009 nach dem sogenannten Schichtenmodell. Zur Berechnung und den Problemen mit einer auf diese Weise ermittelten Lösungsquote sei auf Uhly (2015) verwiesen.

2 Die Analyse der Entwicklung der Vertragslösequote seit 1993 unterliegt der Einschränkung, dass die Methode zur Ermittlung der Vertragslösequoten im Zuge der Umstellung von der Aggregatdatenstatistik auf die vertragsbezogene Einzeldatenerhebung verändert wurde. Insofern ist die Vergleichbarkeit der Daten eingeschränkt, jedoch lassen sich die dargestellten Differenzen nicht über diese methodische Umstellung erklären (Lange 2019; Uhly 2015). 
(2015, 130) konnten zeigen, dass die sehr gute Arbeitsmarktlage 2008 „deutlich vertragslösungserhöhende Effekte in der Probezeit" und nach der Probezeit „risikoerhöhende Effekte bei ausländischen Auszubildenden“ nach sich zog. Der Anstieg der Lösungsquote in den 1980er Jahren von rund 13\% in 1979 auf knapp 25\% in 1992 erfolgte jedoch weitgehend unabhängig von den Veränderungen auf dem Ausbildungsstellenmarkt (Uhly 2015; Weiss 2002).

- Soziologisch werden vorzeitige Vertragslösungen als Folge familiärer Sozialisationsbedingungen und Strategie der Bewältigung von Normenkonflikten (Weiss 1982) sowie als Ergebnis sozialer Selektion und Allokation an der ersten Schwelle (Berufswahl und Rekrutierung) (Büchler 2012) erklärt.

- Psychologisch wird das Phänomen vorzeitiger Vertragslösungen über bestimmte Persönlichkeitsmerkmale der Auszubildenden erklärt, z. B. Entwicklungskrisen (Busemann 1959) und kognitive Fähigkeiten, gemessen bspw. am Schulabschluss (Deuer 2006). Anzunehmen ist, dass auch affektive Persönlichkeitsmerkmale (etwa im Zusammenhang mit der Ausbildungsreife, Engin 2016) oder sensomotorische Fertigkeiten einen Einfluss auf vorzeitige Vertragslösungen haben; hierzu stehen empirische Nachweise jedoch noch aus.

- Aus berufspädagogischer Perspektive werden vorzeitige Vertragslösungen über die Bedingungen der Berufswahl und Berufsorientierung sowie der Ausbildung selbst (z. B. Ausbildungsqualität) erklärt (Lange 2019).

Empirisch zeigt sich, dass das Vertragslösegeschehen komplex und mehrdimensional ist und daher ein Erklärungsansatz allein nicht ausreicht (u. a. Büchler 2012; Deuer 2006; Rohrbach-Schmidt \& Uhly 2015). Vorzeitige Vertragslösungen werden daher zunehmend als prozesshaftes Phänomen konstruiert, das nicht nur eine Ursache hat, sondern von mehreren, unterschiedlichen Faktoren ausgelöst und beeinflusst wird. Einen Vorschlag zur Modellierung des Prozesses legt Klaus (2014) vor. Er charakterisiert das Phänomen einer vorzeitigen Vertragslösung durch mehrere Phasen, in denen Schlüsselereignisse den Prozess auslösen oder vorantreiben: Ausgangspunkt ist das Erleben einer fehlenden Passung infolge des Verlustes von sinn- und identitätsstiftenden Momenten in der Ausbildung. Diese ersten Problemlagen initiieren ein Abwägen und eine Bearbeitung der Problemlagen, mit dem Ziel, Sinnhaftigkeit und Identität wiederherzustellen. Gelingt die Bearbeitung der Problemlagen nicht, verliert der Betroffene zunehmend an Handlungsfähigkeit und fühlt sich überfordert. Als Reaktion darauf konzentrieren sich die Jugendlichen auf andere Lebensbereiche und verlieren die positiven Assoziationen mit der Ausbildung. Die Situation spitzt sich bis zur Entscheidung für eine vorzeitige Vertragslösung zu. Ab dann gibt es keine Möglichkeit mehr, das Problem zu bearbeiten; 
es beginnt die Zeit des Ausharrens bis zur Umsetzung der Entscheidung. Genutzt wird diese Zeit entweder für die Suche nach Alternativen oder die Zentrierung auf andere Lebensbereiche. Abgeschlossen wird der Prozess mit der Umsetzung der vorzeitigen Vertragslösung und damit dem Ausstieg aus dem unmittelbaren Ausbildungsverhältnis.

Die spezifischen Gründe, Motive und Schlüsselereignisse vorzeitiger Vertragslösungen sind empirisch nur schwer erfassbar. Je nachdem, welche Akteure befragt werden, kommen die Studien zu unterschiedlichen Ergebnissen. Während Ausbildungsbetriebe häufig Gründe anführen, die überwiegend im Verantwortungsbereich der Auszubildenden liegen, geben Auszubildende vor allem Gründe an, die den Ausbildungsbetrieben zuzurechnen sind, wobei jedoch auch eigenes Fehlverhalten eingeräumt wird (zusammenfassend Lange 2019; Uhly 2015). Unter methodischen Gesichtspunkten sind die vorliegenden Ergebnisse vorsichtig zu interpretieren, da sie ausschließlich aus retrospektiven Untersuchen gewonnen wurden, die ein gewisses Potential der gegenseitigen Schuldzuschreibung beinhalten.

Unabhängig von den Ursachen basieren die bisherigen Erklärungsansätze vorzeitiger Vertragslösungen überwiegend auf der Analyse und Interpretation der Vertragslösungen als Phänomen aus einer außenstehenden Perspektive. Zu wenig berücksichtigt wird dabei das „Innenleben“ der Auszubildenden, dem subjektive Verarbeitungsprozesse zugrunde liegen, über die sich vorzeitige Vertragslösungen ebenfalls erklären ließen. Das dürfte auch Ausgangspunkt der Modellentwicklung von Klaus (2014) sein, der mit seinem Prozessmodell der vorzeitigen Vertragslösungen erstmals die individuelle Perspektive der Betroffenen auf das Vertragslösegeschehen in den Mittelpunkt rückt.

\section{Anforderungen von Auszubildenden zu Beginn der Berufsausbildung - Einblick in ein Forschungsprojekt zur Berufsausbildungseingangsphase}

Die frühen vorzeitigen Vertragslösungen waren der Ausgangspunkt für eine empirische Untersuchung zur Eingangsphase in die Berufsausbildung (Lange 2019), die im Anschluss an die Befunde zu vorzeitigen Vertragslösungen und aus forschungspragmatischen Gründen auf das erste Ausbildungsjahr ${ }^{3}$ bezogen wurde. In der Berufsbildungsforschung wurde diesem Gegenstandsbereich bisher zu wenig Aufmerksamkeit gewidmet (Kutscha 2006). Insbesondere vor

3 Die Berufsausbildungseingangsphase wurde damit entgegen bisheriger Analysen (Ernst 1997; Kutscha et al. 2009), die sie auf das erste Ausbildungshalbjahr begrenzten, auf einen längeren Zeitraum bezogen. 
dem Hintergrund der Individualisierung als zunehmend dominierende Sozialform und der damit verbundenen Verantwortung des Einzelnen für seine eigene (Berufs-)Biografie (Beck 1996) gewinnt dabei ein subjektorientierter Zugang eine besondere Bedeutung. Solche Zugänge betonen die ,,aktive Rolle des Subjekts bei der Gestaltung sozialer Wirklichkeit“ (Flick et al. 2005, 107). Insofern verdienen die aus der Sicht der Auszubildenden während der Eingangsphase subjektiv wahrgenommenen und erlebten Probleme und Belastungen“" besondere Aufmerksamkeit (Kutscha 2009).

Der als Berufsausbildungseingangsphase bezeichnete Anfang der Berufsausbildung wird als eigene Episode der Transition von der Schule in die Berufsausbildung aufgefasst und im Sinne der Transitionsforschung als Eintritt in einen neuen, routinierten Zusammenhang interpretiert, dem ,bedeutende Veränderungen im Kernland unseres Selbst“ folgen (Parkes 1974, 13). Diese Veränderungen erfolgen durch Lern- und Entwicklungsprozesse, die durch die Anforderungen und Erwartungen im neuen, für den Auszubildenden unbekannten Lebenszusammenhang initiiert werden. Im Gegensatz zu anderen Lern- und Entwicklungsprozessen sind Transitionen durch „verdichtete und akzelerierte Veränderungsphasen“(Welzer 1990, 37) gekennzeichnet, die „,das Subjekt zu neuen Erfahrungen, Wahrnehmungs- und Deutungsweisen“ führen (Welzer 1993, 19). Entgegen der Kausalitäts- und Linearitätsvorstellungen anderer Übergangskonzepte, wird mit dem Transitionsansatz das Ausbalancieren individueller Handlungspotentiale und gesellschaftlicher Handlungsanforderungen betont, das interaktiv und transaktional verläuft und daher viel eher „nach dynamischen und relationalen Referenzen“ (ebd., 37) strukturiert ist, als nach Kausalitätsmustern. Insofern erfordern Transitionen die Bewältigung von Diskontinuitäten auf mehreren Ebenen. Griebel und Niesel (2013) definieren für Bildungsübergänge drei Anforderungsebenen: Entwicklungen finden demnach statt auf einer individuellen Ebene, auf der Veränderungen im Selbstkonzept und im Weltbild des Einzelnen zusammengefasst werden. Auf einer interaktionalen Ebene werden Entwicklungen adressiert, die im Kontext sozialer Beziehungen und Bindungen ausgelöst werden. Die kulturellen Entwicklungsanregungen sowie durch materielle Umgebungsbedingungen ausgelöste Entwicklungen werden auf einer kontextuellen Ebene zusammengefasst.

Die Identitätsentwicklung, das Lernen und die damit verbundenen Verhaltensänderungen werden als Entwicklung im entwicklungspsychologischen Sinne interpretiert. Zur Strukturierung der Entwicklung im Übergang wird auf den ökopsychologischen Ansatz der menschlichen Entwicklung zurückgegriffen, der diese als „Ergebnis der Interaktion zwischen dem wachsenden menschlichen Organismus und seiner Umwelt" (Bronfenbrenner 1989, 32) (Transaktion) versteht. Der Übergang in die duale Berufsausbildung ist mit einer Veränderung der ökologischen Umwelt infolge der Erweiterung der individuellen Lebensbereiche und der Übernahme der Auszubildendenrolle verbunden (Lange 2019). Die damit verbundenen Entwicklungsprozesse sind im 
Sinne Havighursts (1981) durch das Spannungsverhältnis der individuellen Bedürfnisse und Wünsche auf der einen Seite und den gesellschaftlichen und institutionellen Anforderungen auf der anderen Seite geprägt. Diese gesellschaftlichen und institutionellen Anforderungen werden nach dem Anforderungs-Bewältigungs-Paradigma (Lazarus 1995) von den Subjekten vor dem Hintergrund der eigenen Handlungsmöglichkeiten und der Chancen für eine Lösung eingeschätzt und individuell bearbeitet bzw. bewältigt. Misslingt die Bewältigung kann es zu einem Belastungserleben kommen.

Im Fokus der qualitativen Untersuchung zur Berufsausbildungseingangsphase (Lange 2019) standen die individuell von den Auszubildenden in der Berufsausbildungseingangsphase wahrgenommenen Anforderungen und deren Bewältigung sowie das Belastungserleben innerhalb der Berufsausbildungseingangsphase. Ziel der Untersuchung war es, deskriptives und kausales Wissen über die bisher in der Forschung zu wenig berücksichtigte Phase des Eintritts in die duale Berufsausbildung aus Perspektive der Auszubildenden am Beispiel des Handwerks zu gewinnen und damit die auf kaufmännische Berufe ausgerichtete Untersuchung von Kutscha und Kollegen (2009) zu ergänzen.

Die Datenerhebung erfolgte mittels problemzentrierter Interviews in zwei Interviewwellen (nach dem ersten Ausbildungshalbjahr und nach dem ersten Ausbildungsjahr). Insgesamt konnten 18 Auszubildende der Ausbildung im Kraftfahrzeugmechatronikerhandwerk für die erste Interviewwelle gewonnen werden, von denen mit 11 Auszubildenden auch in der zweiten Interviewwelle ein Interview durchgeführt werden konnte. Die gewonnenen und transkribierten Daten wurden mittels verschiedener Verfahren der qualitativen Inhaltsanalyse (induktiv-deduktive Kategorienbildung, inhaltlich-strukturierende Analyse, skalierende Analyse) ausgewertet und interpretiert (Lange 2019).

Im Ergebnis zeichnet das Projekt ein differenziertes Bild der Auszubildenden auf ihre Ausbildung nach, das entsprechend des theoretischen Ansatzes durch die Anforderungen und Diskontinuitäten zu Beginn der Berufsausbildung geprägt ist. Die von den Auszubildenden wahrgenommenen Anforderungen sind erwartungsgemäß vielfältig. Grundlegende Annahme der Analyse und Strukturierung der Anforderungen war es, dass die Wahrnehmung der Anforderungen und der damit verbundenen Lern- und Entwicklungsanlässe von einer persönlichen Sinnhaftigkeit abhängt, die sich auf die „Bedrohung selbstrelevanter Sollwerte in Form von zentralen psychischen Motiven und Bedürfnissen“ (Kaluza 2015, 36f.) zurückführen lässt. Damit wird auf eine grundlegende Annahme der Motivationspsychologie und Bedürfnisforschung rekurriert, nach der Bedürfnisse das intrapersonelle Geschehen bestimmen und Auslöser von Handlungsmotivation sind (Mägdefrau 2006). Insofern wurden die von den Auszubildenden individuell wahrgenommenen Anforderungen als individuelle, unerfüllte Bedürfnisse der Auszubildenden interpretiert und im Anschluss an das Konzept der psychologischen Grundbedürfnisse nach Maslow 
$(1990)^{4}$ auf fünf Anforderungsebenen verdichtet, innerhalb derer sich verschiedene Anforderungen an die individuelle Entwicklung der Jugendlichen, ihre soziale Einbindung und die Erschließung des neuen Lebensraums der Ausbildung (Griebel \& Niesel 2013) bündeln:

- Auf der physiologischen Anforderungsebene wurden alle Anforderungen zusammengefasst, die von den Jugendlichen konstitutionelle Anpassungsleistungen erfordern. Charakteristisch dafür sind zeitliche und physische Anpassungserfordernisse, die sich auf die Bedürfnisse der Jugendlichen nach Erholung und Schlaf sowie körperliche Fitness zurückführen lassen. Beispielsweise berichteten die Jugendlichen in den Interviews, dass sie Probleme mit dem zeitigen Aufstehen oder dem langen Stehen im Ausbildungsbetrieb haben.

- Die Ebene der Sicherheitsanforderungen umfasst solche Anforderungen, die den Umgang mit Unsicherheiten und Gesundheitsgefährdungen erfordern, die dem Bedürfnis nach Sicherheit und Gesundheit entgegenstehen. Dazu zählen etwa der Umgang mit körperlichen Beeinträchtigungen im Sinne körperlicher Einschränkungen aufgrund körperlicher Probleme oder Erkrankungen oder die Verarbeitung starker Emotionen wie Unsicherheit und Angst.

- Anforderungen, die sich auf das soziale Umfeld der Auszubildenden beziehen, wurden auf der Ebene der sozialen Anforderungen gebündelt. Das soziale Umfeld wird in vielerlei Zusammenhängen als bedeutender Faktor der Berufsausbildung herausgestellt, etwa wird sein Einfluss auf die Ausbildungszufriedenheit, auf die Bewertung der Ausbildungsqualität und auf das Risiko vorzeitiger Vertragslösungen herausgearbeitet. Auch in den der Untersuchung zugrunde liegenden Daten zeigten sich eine Reihe von sozialen Anforderungen, wie etwa die soziale Integration in die Arbeitsgruppe oder die Bewältigung von Konflikten, die sich nicht immer mit dem Bedürfnis der Jugendlichen nach sozialer Einbindung und Anerkennung in Einklang bringen lassen.

- Auf der Ebene der Kompetenzanforderungen wurden alle Anforderungen zusammengefasst, die sich auf die Kompetenzentwicklung der Jugendlichen beziehen. Wie auch in anderen Untersuchungen haben die Jugendlichen hohe Erwartungen bzw. Bedürfnisse an ihre Kompetenzen und ihre Kompetenzentwicklung, die in der Berufsausbildungseingangsphase nicht immer erfüllt werden können. Auch das Fehlen von Lerngelegenheiten, sei es durch die Qualität der Vermittlungsprozesse

4 Die Maslow'sche Bedürfnistheorie ist zwar nicht unumstritten (zusammenfassend Mägdefrau 2006), doch hat sie bis heute eine hohe Bedeutung in der Arbeitszufriedenheitsforschung und gilt auch in der Bedürfnisforschung als eines ,,der zentralen Erklärungsmodelle menschlichen Bedürfnisgeschehens“" (Mägdefrau 2006, 42). 
oder das Verrichten ausbildungsfremder Tätigkeiten, belastet die Auszubildenden, da es ihrem Bedürfnis nach Kompetenz und Kompetenzentwicklung entgegensteht.

- Auch ein Bedürfnis nach Autonomie und Selbstbestimmung verspüren zumindest einzelne Auszubildende in der Berufsausbildungseingangsphase. So berichten die Jugendlichen etwa über Belastungen durch Kontrollen und vorgeschriebene Arbeitsabläufe (Lange 2019).

Zwischen den einzelnen Anforderungsebenen bestehen Beziehungen, darauf deuten die Daten hin. Dies zeigt sich beispielsweise darin, dass der Befriedigung physiologischer Bedürfnisse zumindest bis zu einem bestimmten Befriedigungsgrad mehr Bedeutung beigemessen wird, als der Befriedigung anderer Bedürfnisse. Ein Auszubildender berichtet etwa, dass er nach der Arbeit eher Erholung und Schlaf bevorzugt, als etwas mit seinen Freunden zu unternehmen und damit soziale Kontakte zu pflegen, obwohl ihm die sozialen Kontakte fehlen (ebd.). Es deutet sich an, dass die von Maslow (1990) postulierte Annahme der Hierarchisierung der psychologischen Grundbedürfnisse durchaus auch in der Strukturierung der Berufsausbildungseingangsphase relevant wird. Dies zeigt sich allerdings lediglich für die Ebene der physiologischen Anforderungen und der Sicherheitsanforderungen, die überwiegend zu Beginn der Ausbildung wahrgenommen und im ersten Interview (nach dem ersten Ausbildungshalbjahr) überwiegend nur retrospektiv thematisiert wurden. Vor allem die Ebenen der sozialen Anforderungen und der Kompetenzanforderungen bestimmen die Berufsausbildungseingangsphase. Auf diesen Anforderungsebenen erleben die Auszubildenden vielfältige Diskontinuitäten, die mit hohen Belastungen einhergehen.

Im Ergebnis wird die Berufsausbildungseingangsphase als konflikt- und stressreiche Entwicklungsphase der Auszubildenden entfaltet, die durch die individuellen Bedürfnisse der Auszubildenden gekennzeichnet ist. Zu diesen Bedürfnissen zählen: sich bzw. die eigene physiologische Konstitution dem neuen Kontext anzupassen (physiologische Bedürfnisse), sich sicher zu fühlen und Ambiguität zu reduzieren (Sicherheitsbedürfnisse), sich in das neue soziale Gefüge zu integrieren (soziale Bedürfnisse), die eigene Handlungsfähigkeit (wieder-)herzustellen (Kompetenzbedürfnisse) und sich selbst $\mathrm{zu}$ entfalten (Selbstverwirklichungsbedürfnisse). Gerade zu Beginn der Ausbildung werden die individuellen Bedürfnisse der Jugendlichen erschüttert, weshalb sie versuchen, die Bedürfnisbefriedigung wiederherzustellen. Dabei entstehen für die Auszubildenden Belastungen, die individuell verarbeitet und bewältigt werden müssen. Wie in anderen Untersuchungen auch, konnte eine hohe Motivation der Auszubildenden, die mit der Ausbildung verbundenen Anforderungen zu bewältigen, rekonstruiert werden. Einzelne, punktuelle Unzufriedenheit und Überforderungen scheinen dabei keinen Einfluss auf die Bewertung der Ausbildung zu haben, erst eine Vielzahl belastender Umstände und 
ein intensives Ungleichgewicht der individuellen Bedürfnisse und ihrer Befriedigungsmöglichkeiten führen scheinbar zum Verlust der sinn- und identitätsstiftenden Wirkung der dualen Berufsausbildung (Lange 2019).

\section{Vorzeitige Vertragslösungen aus subjektiver Perspektive der Auszubildenden - Schlussfolgerungen und Ausblick}

Vorzeitige Vertragslösungen gehen in etwa der Hälfte der Fälle von den Auszubildenden aus, insofern ist zur Erklärung des Phänomens die individuelle Perspektive der betroffenen Jugendlichen auf das Vertragslösegeschehen durchaus als relevant zu bewerten. In bisherigen Erklärungsansätzen wird jedoch kaum auf das subjektive Erleben des Ausbildungsgeschehens und der vorzeitigen Vertragslösungen rekurriert, Vertragslösungen werden eher durch äußere Einflussfaktoren und individuelle Merkmale der Jugendlichen erklärt. Die individuelle Perspektive der Jugendlichen wird vor allem durch die retrospektive Rekonstruktion von Motiven und Gründen vorzeitiger Vertragslösungen berücksichtigt, wobei auch hierbei nicht das subjektive Erleben der Ausbildung und die Verarbeitungsprozesse der Auszubildenden im Analysefokus stehen, sondern einzelne (isolierte) Ursachen und Gründe vorzeitiger Vertragslösungen gesucht werden. Mit dem Prozessmodell von Klaus (2014) rückt die individuelle Wahrnehmung der Auszubildenden in Bezug auf den Ausbildungsprozess in den Analysefokus vorzeitiger Vertragslösungen. Im Gegensatz zu anderen Erklärungsansätzen wird der prozesshafte Charakter und die Multidimensionalität der vorzeitigen Vertragslösungen berücksichtigt. Vorzeitige Vertragslösungen werden erklärt als zunehmender Verlust der Handlungsfähigkeit der betroffenen Auszubildenden und eine zunehmend nachlassende sinn- und identitätsstiftende Wirkung der Ausbildung (vgl. Abschnitt 2).

Für die Frage, wie es zu einer nachlassenden sinn- und identitätsstiftenden Wirkung der Ausbildung kommen kann, liefert das im Abschnitt 3 vorgestellte Projekt bzw. das im Rahmen des Projekts entworfene Modell der Berufsausbildungseingangsphase fruchtbare Ansätze. Bereits im Rahmen der Untersuchung musste festgestellt werden, dass die identifizierten Anforderungen sich nicht auf die Berufsausbildungseingangsphase beschränken, sondern zu erwarten ist, dass diese auch über die Berufsausbildungseingangsphase hinweg relevant werden (bspw. Lange 2019). Insofern ist durchaus zu hinterfragen, ob das vorgelegte Modell auf die Berufsausbildungseingangsphase beschränkt ist oder eine Relevanz über die gesamte Ausbildungsdauer aufweist. Wird der Übergang in die Berufsausbildung im Anschluss an van Gennep (1986) als spezifische Phase eines größeren Übergangs von der Schule in die Berufstätigkeit aufgefasst, würde die Berufsausbildung als sogenannte Schwellen- oder Umwandlungsphase begriffen, in der die Übergänger sich in einer Schwelle 
zwischen dem Nicht-mehr-Schüler (im Sinne des Schülers im vollzeitschulischen, allgemeinbildenden Schulsystem) und dem Noch-nicht-Facharbeiter befinden. Ganz im Sinne Turners befinden sie sich in dieser Phase „weder hier noch da; sie sind weder das eine noch das andere, sondern befinden sich zwischen den vom Gesetz, der Tradition, der Konvention und dem Zeremonial fixierten Positionen“ (1989, 5). Insofern ließe sich erklären, warum die mit dem Übergang verbundenen Anforderungen der Berufsausbildungseingangsphase nur schwer von anderen Anforderungen der Ausbildung abgegrenzt werden können. Gleichwohl hieße das, dass eine Übertragbarkeit des Modells auf die gesamte Ausbildungsphase durchaus begründet wäre. Insofern kann das Modell durchaus als fruchtbarer Ansatz zur Erklärung vorzeitiger Vertragslösungen herangezogen werden.

Erklärt werden können vorzeitige Vertragslösungen aus der subjektiven Perspektive der Auszubildenden dann als Überforderung durch unzureichende individuelle Bedürfnisbefriedigung und das zunehmende Unvermögen, die Bedürfnisbefriedigung wiederherzustellen. Im Sinne der dem Modell zugrunde liegenden entwicklungspsychologischen Grundlagen, vor allem dem Anforderungs-Bewältigungs-Paradigma nach Lazarus (1995), lösen die unerfüllten Bedürfnisse kognitive Bewertungsprozesse aus, in denen sowohl der potentielle Stressauslöser (das unerfüllte oder bedrohte Bedürfnis) als auch die (wahrgenommenen) Bewältigungsmöglichkeiten individuell eingeschätzt und bewältigt werden und je nach Ergebnis der kognitiven Einschätzungsprozesse ein Belastungserleben entsteht. Insofern initiieren unerfüllte oder bedrohte Bedürfnisse in der Ausbildung Belastungen, die von den Auszubildenden mit den ihnen zur Verfügung stehenden und den individuell wahrgenommenen Ressourcen zu bewältigen sind, um die Befriedigung der eigenen Bedürfnisse sicherzustellen. Die Entscheidung für eine Vertragslösung ist dann als individuelle Bewältigungsstrategie der Jugendlichen zu verstehen, um die mit den nicht erfüllten oder bedrohten Bedürfnissen verbundenen Belastungen zu reduzieren und damit die individuelle Bedürfnisbefriedigung wiederherzustellen.

Die vorzeitige Vertragslösung wird dabei, wie bei Klaus (2014), als lang andauernder, iterativer Prozess aufgefasst, der durch zunehmende Belastung und Überforderung und einer dadurch wachsenden, individuell wahrgenommenen Handlungsunfähigkeit des Jugendlichen zugespitzt wird. Die Einflussfaktoren, die in anderen Ansätzen zur Erklärung vorzeitiger Vertragslösungen herangezogen werden, beeinflussen den Bewältigungsprozess bzw. die individuell wahrgenommenen Bewältigungsmöglichkeiten ebenso, wie die individuellen Verarbeitungs- und Deutungsweisen der Jugendlichen. Beispielsweise würde die von den Auszubildenden individuell wahrgenommene Ausbildungsmarktsituation als Bewältigungsmöglichkeit interpretiert werden, die die Entscheidung für eine vorzeitige Vertragslösung als Bewältigungsstrategie beeinflusst. 
Mit diesem Ansatz ließe sich auch erklären, warum die Gedanken an vorzeitige Vertragslösungen nicht zwangsweise zu vorzeitigen Vertragslösungen führen bzw. die Gedanken an vorzeitige Vertragslösungen keinen guten Prädiktor vorzeitiger Vertragslösungen darstellen (u. a. Deuer 2006). Ebenso wie die Entscheidung für eine vorzeitige Vertragslösung wären Gedanken an vorzeitige Vertragslösungen eine Strategie zur Bewältigung der mit den unerfüllten Bedürfnissen verbundenen Belastungen. Im Gegensatz zu der problemorientierten (aktiven) Bewältigungsstrategie der vorzeitigen Vertragslösung zielen Gedanken an vorzeitige Vertragslösungen auf die emotionale Regulation der individuellen Stressreaktion, die der Literatur nach vorrangig dann angewandt werden, wenn die betroffene Person das Gefühl hat, die Situation nicht (mehr) kontrollieren zu können und ertragen zu müssen (Reimann \& Pohl 2006).

Mit dem dargestellten Erklärungsansatz rücken individuelle Wahrnehmungs-, Deutungs- und Verarbeitungsprozesse der Jugendlichen in den Fokus der Analysen vorzeitiger Vertragslösungen. Die Potentiale des Modells zur Erklärung vorzeitiger Vertragslösungen beschränken sich jedoch auf die Perspektive der Auszubildenden, da das Modell aus einem subjektbezogenen Übergangsverständnis aus der Perspektive der Übergänger abgeleitet wurde. Eine Übertragbarkeit des Modells auf Ausbilder oder ausbildende Personen als ebenfalls am Übergang beteiligte Akteure ist nicht möglich, da der Übergang für diese Personen, die sich bereits in dem von den Auszubildenden neu zu erschließenden Lebensraum befinden, keine „bedeutenden Veränderungen im Kernland [ihres] Selbst“" (Parkes 1974, 13) erfordert. Aus der Perspektive der Übergangsforschung sind diese Personen als Bewältigungsressource zu interpretieren, die von den Auszubildenden mehr oder weniger auch als solche wahrgenommen werden. Zur Erschließung vorzeitiger Vertragslösungen aus der Perspektive der Ausbildenden eignet sich das Modell daher nicht, hierfür sind andere Analyseansätze, beispielsweise aus einer systemtheoretischen Perspektive (Bührmann 2008), zu erschließen.

Der skizzierte Ansatz stellt Überlegungen im Anschluss an das in Abschnitt 3 vorgestellte Projekt und die Befunde zur Berufsausbildungseingangsphase dar. Um die tatsächliche Erklärungskraft des Modells bewerten und darauf aufbauende berufsbildungspraktische Implikationen formulieren zu können, bedarf es empirischer, numerisch repräsentativer Analysen des Modells und der Übertragbarkeit des Modells auf das Phänomen vorzeitiger Vertragslösungen. Insofern stellt der Ansatz einen theoretischen Beitrag zum berufsund wirtschaftspädagogischen Diskurs um vorzeitige Vertragslösungen dar, der auf empirische Befunde und theoretische Ansätze der Übergangsforschung und der Entwicklungspsychologie aufbaut. 


\section{Literatur}

Allianz für Aus- und Weiterbildung (2019). Allianz für Aus- und Weiterbildung 2019 2021. Online: https://www.aus-und-weiterbildungsallianz.de/AAW/Redaktion/DE/Downloads/allianz-fuer-aus-und-weiterbildung-2019-2021.pdf?_blob= publicationFile\&v=3 (02.12.2019).

Beck, U. (1996). Risikogesellschaft. Auf dem Weg in die Moderne. Edition Suhrkamp 3326. Frankfurt a. M. u. a.: Suhrkamp.

Bundesinstitut für Berufsbildung (BIBB) (2017). Datenreport zum Berufsbildungsbericht 2017. Informationen und Analysen zur Entwicklung der beruflichen Bildung. Bielefeld: Bertelsmann.

Bundesministerium für Bildung und Forschung (BMBF) (2019). Berufsbildungsbericht 2019. Bonn: BMBF.

Bronfenbrenner, U. (1989). Die Ökologie der menschlichen Entwicklung. Natürliche und geplante Experimente. Fischer-Taschenbücher. Frankfurt a. M. u. a: Fischer.

Büchler, J. (2012). Sekundärstatistische Analyse von Abbrecherdaten aus soziologischer Perspektive zur Abbruchprävention. In C. Baumeler, B.-J. Ertelt \& A. Frey (Hrsg.), Diagnostik und Prävention von Ausbildungsabbrüchen in der Berufsbildung. Programm für lebenslanges Lernen (Praelab) (S. 74-80). Landau: Empirische Pädagogik (Bildung, Arbeit, Beruf und Beratung, 1).

Bührmann, T. (2008). Übergänge in sozialen Systemen. Weinheim u. a.: Beltz.

Busemann, A. (1959). Krisenjahre im Ablauf der menschlichen Jugend (2. Aufl.). Ratingen: Aloys Henn.

Deuer, E. (2006). Früherkennung und Prävention von Ausbildungsabbrüchen - Ursachenanalyse, Handlungsfelder und relevante Akteure. Empirische Pädagogik, 30(3/4), 453-474.

Engin, G. (2016). Berufliche Aspirationen von Jugendlichen der gymnasialen Mittelstufe. Eingrenzung und Konstitution im Kontext von persönlicher Bindung, beruflicher Exploration und beruflicher Identität. Online: https://tuprints.ulb.tu-darmstadt.de/5695/1/Berufliche\%20Aspirationen\%20von\%20Jugendlichen $\% 20 \mathrm{der} \% 2$ 0gymnasialen $\% 20$ Mittelstufe $\% 20 \% 282016 \% 29 \% 2 \mathrm{C} \% 20$ Dissertation $\% 2 \mathrm{C} \% 20$ Gaby\%20Engin\%20\%28geb\%20Steinritz\%29.pdf (02.12.2019).

Ernst, C. (1997). Berufswahl und Ausbildungsbeginn in Ost- und Westdeutschland. Eine empirisch-vergleichende Analyse in Bonn und Leipzig. Bielefeld.

Fischer, A. (2002). Erfahrungen der vom Ausbildungsabbruch betroffenen Personengruppen. In S. Bohlinger \& K. Jenewein (Hrsg.), Ausbildungsabbrecher - Verlierer der Wissensgesellschaft? Konzepte, Risiken und Chancen aktueller Handlungsansätze aus der Berufsbildungsforschung und -praxis. Beiträge zu den 12. Hochschultagen Berufliche Bildung 2002 in Köln (S. 17-26). Bielefeld: Bertelsmann (Berufsbildung in der Wissensgesellschaft. Globale Trends - Notwendige Fragen Regionale Impulse, 22).

Flick, U., von Kardoff, E. \& Steinke, I. (2005). Theorie qualitativer Forschung. Einleitung. In U. Flick, E. von Kardoff \& I. Steinke (Hrsg.), Qualitative Forschung. Ein Handbuch (4. Aufl., S. 106-109). Reinbek: Rowohlt.

Griebel, W. \& Niesel, R. (2013). Übergänge verstehen und begleiten. Transitionen in der Bildungslaufbahn von Kindern. Berlin: Cornelsen. 
Havighurst, R. J. (1981). Developmental tasks and education (3 ${ }^{\text {rd }}$ ed.). New York: Longman.

Jasper, G, Richter, U. A., Haber, I. \& Vogel, H. (2009). Ausbildungsabbrüche vermeiden - neue Ansätze und Lösungsstrategien. Berufsbildungsforschung 6. Bielefeld: Bertelsmann.

Kaluza, G. (2015). Stress - was ist das eigentlich? Wissenschaftliche Stresskonzepte. Berlin, Heidelberg: Springer.

Klaus, S. (2014). Ausbildungsabbruch und Biographie. Über Prozesse, Mechanismen und Wechselwirkungen in Lebensverläufen von Personen mit vorzeitiger Vertragsauflösung in der Berufsausbildung. Frankfurt a. M. u. a.: Peter Lang.

Kutscha, G. (2006). Aller Anfang ist schwer. Einstieg in die Berufsausbildung als Entwicklungsaufgabe beruflicher Kompetenz- und Identitätsentwicklung. In H.E. Tenorth (Hrsg.), Beruf und Berufsbildung. Situation, Reformperspektiven, Gestaltungsmöglichkeiten (S. 85-100). Weinheim u. a.: Beltz.

Kutscha, G. (2009). Einleitung. In G. Kutscha, A. Besener \& S. O. Debie (Hrsg.), Probleme der Auszubildenden in der Eingangsphase der Berufsausbildung im Einzelhandel-ProBE. Abschlussbericht zum Forschungsprojekt (S. 1-8). Duisburg.

Kutscha, G., Besener, A. \& Debie, S. O. (2009). Probleme der Auszubildenden in der Eingangsphase der Berufsausbildung im Einzelhandel - ProBE. Abschlussbericht zum Forschungsprojekt. Duisburg.

Lange, S. (2019). Die Berufsausbildungseingangsphase. Anforderungen an Auszubildende und ihre Bewältigungsstrategien am Beispiel des Kfz-Mechatronikerhandwerks. Bielefeld: wbv (Berufsbildung, Arbeit und Innovation, 55).

Lazarus, R. S. (1995). Stress und Stressbewältigung - ein Paradigma. In S.-H. Filipp (Hrsg.), Kritische Lebensereignisse (S. 198-232). Weinheim: Beltz.

Mägdefrau, J. (2006). Bedürfnisse und Pädagogik. Eine Untersuchung an Hauptschulen. Bad Heilbrunn: Kinkhardt.

Maslow, A. H. (1990). Psychologie des Seins. Ein Entwurf(3. Aufl.). Frankfurt a. M. u. a.: Fischer.

Parkes, C. M. (1974). Vereinsamung. Die Lebenskrise bei Partnerverlust. Psychologisch-soziologische Untersuchung des Trauerverhaltens. Reinbek: Rowohlt.

Piening, D., Hauschildt, U. \& Rauner, F. (2010). ...eigentlich wäre der Abbruch nicht notwendig gewesen - Lösung von Ausbildungsverträgen aus Sicht von Auszubildenden und Betrieben. Eine Studie im Auftrag der Industrie- und Handelskammer Osnabrück-Emsland: Univ. Bremen.

Reimann, S. \& Pohl, J. (2006). Stressbewältigung. In B. Renneberg \& P. Hammelstein (Hrsg.), Gesundheitspsychologie (S. 217-227). Berlin, Heidelberg: Springer.

Rohrbach-Schmidt, D. \& Uhly, A. (2015). Determinanten vorzeitiger Lösungen von Ausbildungsverträgen und berufliche Segmentierung im dualen System. Eine Mehrebenenanalyse auf Basis der Berufsbildungsstatistik. Kölner Zeitschrift für Soziologie und Sozialpsychologie, 67, 105-135.

Turner, V. (1989). Das Ritual. Struktur und Anti-Struktur. Frankfurt a. M. u. a.: Campus (Theorie und Gesellschaft, 10).

Uhly, A. (2015). Vorzeitige Vertragslösungen und Ausbildungsverlauf in der dualen Berufsausbildung. Forschungsstand, Datenlage und Analysemöglichkeiten auf Basis der Berufsbildungsstatistik. Bonn: BIBB.

van Gennep, A. (1986). Übergangsriten. Les rites de passage (Übers. K. Schomburg \& S. M. Schoburg-Scherff). Frankfurt a. M. u. a.: Campus. 
Weiss, R. (1982). Ausbildungsabbruch im Handwerk. Ursachen und Einflussfaktoren bei der vorzeitigen Lösung von Berufsausbildungsverträgen. Köln: Carl (Berufsbildung im Handwerk: Reihe A).

Weiss, R. (2002). Ausbildungsabbruch - eine Herausforderung für die betriebliche Berufsbildung. In S. Bohlinger \& K. Jenewein (Hrsg.), Ausbildungsabbrecher - Verlierer der Wissensgesellschaft? Konzepte, Risiken und Chancen aktueller Handlungsansätze aus der Berufsbildungsforschung und -praxis. Beiträge zu den 12. Hochschultagen Berufliche Bildung 2002 in Köln (S. 5-16). Bielefeld: Bertelsmann (Berufsbildung in der Wissensgesellschaft. Globale Trends - Notwendige Fragen - Regionale Impulse, 22).

Welzer, H. (1990). Zwischen den Stühlen. Eine Längsschnittuntersuchung zum Übergangsprozeß von Hochschulabsolventen. Weinheim: Dt. Studienverl. (Psychologie sozialer Ungleichheit).

Welzer, H. (1993). Transitionen. Zur Sozialpsychologie biographischer Wandlungsprozesse. Zur Sozialpsychologie biographischer Wandlungsprozesse. Tübingen: diskord. 



\title{
Theoretische Grundlagen und empirische Befunde zum Wissensmanagement in der inklusiven Berufsorientierung
}

\author{
Leonie Bogaczyk, Marie Schröder, Thomas Retzmann und Thomas \\ Bienengräber
}

\section{Die inklusive Berufsorientierung als Forschungsdesiderat}

Seit der Ratifizierung der UN-Behindertenrechtskonvention 2009 avancierte der gemeinsame Unterricht zu einem zentralen Gegenstand pädagogischer Forschung; dagegen blieb die inklusive Berufsorientierung als besonderes Handlungsfeld weitgehend unerforscht (Bienengräber, Greiten \& Retzmann et al. 2019). Dabei stellen sich an allgemein- und berufsbildenden Schulen gleichermaßen neue An- und Herausforderungen, auf die die pädagogischen Fachkräfte nicht hinreichend vorbereitet sind (Dreer 2013). Um auch in Anbetracht der Exklusionsrisiken auf dem Arbeitsmarkt einen gelingenden Übergang in den Beruf ermöglichen zu können, kommt der Professionalität der pädagogischen Fachkräfte in diesem Handlungsfeld jedoch eine noch gröBere Bedeutung zu als ohnehin (Bylinski 2015). Professionalität versteht sich als professionelle Handlungskompetenz (Weinert 2001, Baumert \& Kunter 2006; Blömeke, Kaiser \& Lehmann 2008), unterteilt in motivational-affektive und kognitive Bereiche. Für die kognitive Bewältigung beruflicher Anforderungen ist professionelles, also fundiertes und breit gefächertes sowie schnell verfügbares Wissen erforderlich, um domänenspezifische Probleme innerhalb pädagogischer Handlungsfelder erkennen und lösen zu können (Bach, Schmidt \& Schaub 2016). Im Kontext der inklusiven Berufsorientierung können pädagogische Fachkräfte demnach dann professionell handeln, wenn sie über jene berufliche Handlungskompetenz verfügen und sie nutzen, die - konkretisiert als Wissen, Fähig- und Fertigkeiten sowie Haltungen - zur Bearbeitung von Problemen in der inklusiven Berufsorientierung erforderlich sind. Demnach ist Wissen eine Prämisse professioneller Handlungskompetenz und somit auch des Wissensmanagements im spezifischen Kontext der inklusiven Berufsorientierung. Es ist eine Voraussetzung, um den Anforderungen einer schulischen inklusiven Berufsorientierung gerecht zu werden und im Sinne des ,organisationalen Lernens“" (Pawlowsky 1994; Probst \& Büchel 1994) einen erfolgreichen Übergang von der Schule in den Beruf gestalten zu können.

Das Forschungsfeld der schulischen Berufsorientierung nebst der entsprechenden Qualifizierung von Lehrpersonen sei „fest etabliert“, so Brüggemann, Driesel-Lange und Weyer $(2017,10)$. Jedoch besteht bislang kein Konsens 
über das Ziel der Berufsorientierung. Im „Thüringer Modell“ wird die Förderung der Berufswahlkompetenz als Ziel bestimmt (Driesel-Lange, Kracke \& Hany et al. 2013); Rahn, Brüggemann und Hartkopf (2013) heben auf das „Gelingen der Berufswahl" ab und somit auf den Erfolg des Übergangsprozesses. Auch die Unterstützung seitens der Lehrer*innen wird bereits als eine Gelingensbedingung für einen erfolgreichen Übergang in die Arbeitswelt empirisch exploriert (Mayhack \& Kracke 2010). In Verbindung mit dem Anspruch der Inklusion gibt es jedoch kaum Forschungsanknüpfungspunkte (Koch 2015, Nentwig 2018). Empirische Studien zur inklusiven Berufsorientierung sowie zur Professionalisierung und Qualifizierung (Moser 2016; Bergs \& Niehaus 2016; Buchmann \& Bylinski 2013) stehen noch aus.

Der folgenden Untersuchung liegt ein weites Inklusionsverständnis zugrunde, um nicht bestimmte Gruppen ex ante auszuschließen, die von Benachteiligungen betroffen sein könnten (Hinz 2002, 2009; Sander 2004; Löser \& Werning 2015). Dies wirft die Frage auf, wie auf die damit steigende Heterogenität der Zielgruppe pädagogisch reagiert werden kann. Eine Antwort darauf ist eine individualisierte Berufsorientierung (vgl. im Überblick Brüggemann, Driesel-Lange \& Weyer 2017), die konzeptionell allerdings das gesamte Spektrum der Schüler*innen adressiert. Individualisierungsbemühungen hinsichtlich der Schüler*innen mit sonderpädagogischem Förderbedarf finden sich in der schulischen Praxis beispielsweise im Zusammenhang mit der Suche nach geeigneten Betrieben und Arbeitsplätzen für Betriebspraktika (Greiten, Bienengräber \& Retzmann et al. 2020). Das hier zugrunde gelegte weite Inklusionsverständnis umschließt somit eine Adaption schulischer Konzepte, die über die Gruppe der Schüler*innen mit einem sonderpädagogischen Unterstützungsbedarf hinausweisen, jedoch verändern sich hierdurch die Anforderungen an Lehrpersonen. Aus einer Professionalisierungsperspektive ist bedeutsam, wie die Lehrer*innen (Regelschullehrkräfte und Sonderpädagog*innen) sowie „weitere“ pädagogische Fachkräfte (in der Schule vor allem Sozialpädagog*innen, extern unter anderem Berufseinstiegbegleiter*innen), die im Handlungsfeld der inklusiven Berufsorientierung aktiv sind, diesen fächerübergreifenden Anforderungen gerecht werden können (Dreer 2013). Bezüglich der Professionalität der Lehrer*innen ist zu fragen, wie sie in den drei Phasen der Lehrerbildung gefördert werden sollte. Dies ist das erkenntnisleitende Interesse einer hochschulübergreifenden Studie (BMBFFörderkennzeichen: 01NV1723A/B).

Die beteiligten pädagogischen Fachkräfte können wichtige Hinweise und Handlungsempfehlungen zur schulpraktischen Umsetzung sowie zur Einbettung in die Aus- und Weiterbildung von Lehrkräften geben (Lazarides, Ohlemann \& Wills et al. 2015). Nachfolgend wird der Frage nachgegangen, welche Bedeutung dabei dem Wissensmanagement zukommt. Es werden ausgewählte Aspekte herausgegriffen, die die qualitative Teilstudie als bedeutsam erwies. Zunächst wird ein weit verbreitetes Konzept des Wissensmanagements 
eingeführt und auf das hier interessierende Handlungsfeld übertragen. In einer dichten Beschreibung werden die drei Bausteine »Wissensziele, Wissensidentifikation und Wissenserwerb « näher beleuchtet - zunächst theoretisch, sodann empirisch auf der Grundlage einer qualitativen Exploration des Handlungsfeldes. Es wird erörtert, ob die sich stellenden Anforderungen und Herausforderungen durch ein professionelles Wissensmanagement (besser) bewältigt werden können. Abschließend sollen mögliche Schlussfolgerungen für die Lehrerbildung und Schulentwicklung in Forschung und Praxis abgeleitet werden.

\section{Referenzrahmen für das schulische Wissensmanagement}

Unter »Wissensmanagement « kann - ganz allgemein - die Identifikation aller relevanten Wissenspotenziale und ihre systematische Ausschöpfung durch die Optimierung der Wissensflüsse entlang von Kernprozessen verstanden werden, wie sie unter anderem Probst, Raub und Romhardt (2012) beschreiben. Bereits Chott (1998) stellt die Bedeutung des Wissensmanagements in Schulen heraus, die seither noch zugenommen haben dürfte. Das Bildungssystem kann als gesellschaftliches Subsystem aufgefasst werden, das im Kontext der Berufsorientierung insbesondere mit dem Beschäftigungssystem als weiterem Subsystem der Gesellschaft gekoppelt ist. Als ein zeitlich vorgelagertes Subsystem übt es diesbezüglich eine Qualifikationsfunktion aus. Die Dynamik der gesellschaftlichen Entwicklungsprozesse verlangt nun eine Weiterentwicklung des Subsystems Schule, damit diese ihrem gesellschaftlichen Auftrag entsprechen kann (Wiater 2007). Der geschilderte Zusammenhang lässt sich auch in das Konzept der Schule als einer „lernenden Organisation“ einordnen (Reinmann-Rothmeier 2001). Was unter Wissensmanagement genauer zu verstehen ist, wird im Folgenden begrifflich und modelltheoretisch entfaltet. Zudem werden ausgewählte Kernprozesse beschrieben. Damit wird ein theoretisch fundierter Referenzrahmen aufgespannt, der die systematische Einordnung der in Kapitel 4 vorgelegten empirischen Befunde zum schulischen Wissensmanagement bei der inklusiven Berufsorientierung erlaubt.

\subsection{Zum Begriff des Wissens, das es ,,zu optimieren“ und auszuschöpfen gilt}

Probst, Raub und Romhardt $(2012,22)$ definieren Wissen extensional sehr umfassend und funktional sehr eng als "die Gesamtheit der Kenntnisse und Fähigkeiten, die Individuen zur Lösung von Problemen einsetzen“. Es umfasse theoretische Erkenntnisse, praktische Alltagsregeln und Handlungsanweisungen, stütze sich zwar auf Daten und Informationen, werde jedoch von Individuen konstruiert und repräsentiere ,deren Erwartungen über Ursache- 
Wirkungs-Zusammenhänge“ (Probst, Raub \& Romhardt 2012, 22). Dagegen stellt Baumert $(1998,214)$ nicht auf die Verwendung, sondern auf die Entstehung und den Inhalt ab: Wissen sei „das Kenntnishaben von etwas per Sinneswahrnehmung, per Mitteilung oder durch das Lernen“. Es bezeichne „die Erkenntnis von etwas als das Kennen von Zusammenhängen, des Wesens und der Gründe von Seiendem" (ebd.). Beiden Definitionen ist gemeinsam, dass Wissen als Zustand und kristalline Struktur verstanden wird. Demgegenüber betonen Davenport und Prusak dessen fluiden Charakter: Wissen sei ,eine fließende Mischung aus strukturierten Erfahrungen, Wertvorstellungen, Kontextinformationen und Fachkenntnissen, die in ihrer Gesamtheit einen Strukturrahmen zur Beurteilung und Eingliederung neuer Erfahrungen und Informationen bietet" $(2000,32)$. Wissen wäre demnach also Voraussetzung und Ergebnis strukturierter Informationsverarbeitung zugleich.

Willke (2001) unterscheidet personales Wissen von Einzelpersonen von organisationalem Wissen, das institutionalisiert sei und daher auch als institutionelles oder Systemwissen deklariert wird. Dabei handele es sich um Leitlinien oder Arbeitsprozessbeschreibungen (ebd.; Huber \& Krey 2009). Für Probst, Raub und Romhardt (2012) setzt sich das organisationale Wissen aus individuellen und kollektiven Wissensbeständen zusammen, auf die eine Organisation zur Lösung ihrer Aufgaben zurückgreifen kann. Beiden Differenzierungen gemeinsam ist, dass sie die Träger des Wissens klassifizieren.

Resultat der begrifflichen Auseinandersetzung ist, dass es keine einheitliche Definition gibt. Es ist daher angeraten, der empirischen Exploration einen weiten Begriff von Wissen als Inhalt, Struktur und Prozess zugrunde zu legen, um a priori nichts auszuschließen, was sich a posteriori als wesentlich herausstellen könnte.

\subsection{Charakterisierung des ausgewählten Wissensmanagementmodells}

Für das Wissensmanagement gibt es mehrere Modelle und Konzepte, denen die Intention der Wissensentwicklung und Wissensbewahrung gemeinsam ist und die auch sonst Ähnlichkeiten aufweisen. Schüppel (1996) beschreibt die Prozesse Generierung, Nutzung, Transfer und Institutionalisierung; Davenport und Prusak (2000) nennen die Prozesse Entwicklung, Kodifizierung und Transfer. Als theoretisch fundierter Referenzrahmen soll hier das umfassendere Modell des Wissensmanagements von Probst, Raub \& Romhardt 2012 herangezogen werden, das bereits auf den schulischen Kontext angewandt wurde (Hamm 2004; Sarnitz 2011; Heitmann 2013), jedoch noch nicht auf einen inklusiven Kontext.

Innerhalb eines Regelkreises aus Wissenszielen und Wissensbewertung befinden sich die Kernprozesse Identifikation, Bewahrung, Nutzung, 
Verteilung, Entwicklung und Erwerb von Wissen (Dick \& Wehner 2002). Aus Platzgründen erfolgt hier die Beschränkung auf die Wissensziele, die Wissensidentifikation und den Wissenserwerb; die davon abgrenzbaren Prozesse des Wissensmanagements müssen einer zukünftigen Analyse vorbehalten bleiben. Durch eine dichte Beschreibung der ausgewählten Wissensmanagementbausteine wird allerdings die Grundlage dafür gelegt: Zum einen ist die Explikation der Wissensziele die Voraussetzung der Wissensbewertung, die die Wirksamkeit des Wissensmanagements zum Gegenstand hat; zum anderen setzen die Wissensnutzung und -entwicklung die Wissensidentifikation und Wissensverteilung voraus (Probst, Raub \& Romhardt 2012).

Das Modell erlaubt es, einzelne Bausteine des Wissensmanagements unabhängig voneinander zu analysieren und zu einem späteren Zeitpunkt weitere Wissensbausteine hinzuzunehmen sowie Verbindungen zwischen ihnen zu untersuchen. Im Zuge der weiteren Beforschung des Handlungsfeldes kann dann eine Systematisierung der Funktionen des Wissensmanagements vorgenommen werden. Die Explikation von Wissenszielen ist jedoch grundlegend für eine lösungsorientierte Anwendung des Modells beim schulischen Wissensmanagement und stellt daher auch hier den ersten Schritt dar. In engem Zusammenhang damit steht die Identifikation des Wissens, das für die Erreichung dieser Ziele notwendig ist. Der dritte Kernprozess »Wissenserwerb « muss hier schon allein deshalb besondere Beachtung finden, weil es insgesamt um die Professionalisierung im Rahmen der Lehrerbildung geht.

\subsection{Grundlegende Kernprozesse des Wissensmanagements - restrukturiert}

Probst, Raub und Romhardt (2012) zufolge sind Wissensziele die Grundlage der inhaltlichen Ausgestaltung des Wissensmanagements. Sie stehen für den Soll-Zustand des Wissens. Der Ist-Zustand wird durch die Wissensidentifikation erhoben. Etwaige Soll-Ist-Abweichungen (fehlendes Wissen) sollen durch den Prozess des Wissenserwerbs abgebaut werden. Zusammen können diese drei Bausteine als Lernprozess aufgefasst werden (siehe Abb. 1):

(1) Wissensziele: Die zentralen Wissensziele in einem Handlungsfeld legen fest, wozu die Wissensbestände, hier der Lehrer*innen sowie der weiteren pädagogischen Fachkräfte aufgebaut werden sollen. Die Identifikation von Wissenszielen bietet Hinweise darauf, in welche Richtung und bis zu welchem Punkt deren Qualifizierung in den drei Phasen der Lehrerbildung erfolgen sollte. Nur wenn eine konkrete Zielbestimmung vorliegt, kann das organisationale Lernen effektiv gestaltet werden.

(2) Wissensidentifikation: In diesem Prozess wird nicht nur erfasst, welche Daten, Informationen und Fähigkeiten intern vorliegen, sondern auch das Wissensumfeld der Organisation analysiert; darüber hinaus, welches interne und externe Wissen nicht vorhanden ist. Indem Wissensbestände und -defizite 
offengelegt werden, wird Transparenz geschaffen. Ferner wird beleuchtet, welche kollektiven Fähigkeiten sich in diesem Handlungsfeld explorieren lassen oder welche Experten wichtige Wissensträger sind.

(3) Wissenserwerb: Der Wissenserwerb umfasst externe Quellen, die relevantes Wissen für die Organisation besitzen und weitergeben. Der Austausch zwischen Akteuren spielt eine wichtige Rolle, etwa durch gemeinsame Aufgaben als Prozesse und den damit verbundenen Austausch von Expertenwissen. Es ist aber auch zu eruieren, welches vorhandene Wissen intern erweitert bzw. vertieft werden könnte. Übertragen auf das vorliegende Handlungsfeld ist zu fragen, welches professionsbezogene Wissen (und Können) in der Lehrerbildung erworben wird und wie Lehrer*innen sowie weitere pädagogische Fachkräfte ihr problemlösungsrelevantes, funktionales Wissen (und Können) im Handlungsfeld der inklusiven Berufsorientierung entwickeln.

Abb. 1: Schulisches Wissensmanagement als Lernprozess

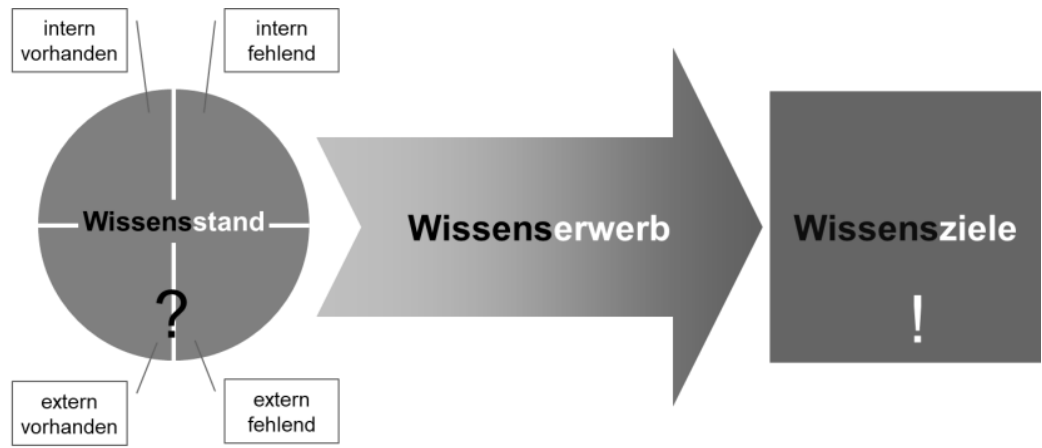

Quelle: eigene Abbildung

\section{Empirische Ergebnisse zum Wissensmanagement in der inklusiven Berufsorientierung}

Da das Feld der inklusiven Berufsorientierung nahezu unerforscht ist, wurde ein explorativer Zugang gewählt. Zur Datenerhebung wurden von Mai bis September 2018 insgesamt 22 Gruppendiskussionen im Setting multiprofessioneller Expertenworkshops geführt. Unter den Teilnehmer*innen waren unterschiedliche Professionen und Institutionen vertreten. Neben pädagogischen Fachkräften (darunter Regelschullehrkräfte, Sonder- und Sozialpädagog*innen) von Schulen der Sekundarstufen in Nordrhein-Westfalen nahmen unter anderem auch Akteur*innen der regionalen Bundesagenturen sowie diverser 
Ämter und Verbände teil. In den Gruppendiskussionen wurden je vier bis sechs Personen zusammengeführt, wobei auf eine möglichst große Heterogenität der Institutionen geachtet wurde.

Der nach Lamnek (2005) für Gruppendiskussionen erforderliche gemeinsame Erfahrungsraum bestand in Erfahrungen in der inklusiven Berufsorientierung aus Sicht der jeweiligen Institution bzw. Profession. Alle im Folgenden wiedergegebenen Aussagen der Proband*innen müssen vor diesem Hintergrund verstanden werden, auch wenn diese über den eingegrenzten Gegenstandsbereich der inklusiven Berufsorientierung hinauszuweisen scheinen. Als Impuls zur Stimulation der Diskussion wurden je zwei bis drei Leitfragen zu Themen der Erhebungseinheiten gestellt. Diese Diskussionen dauerten zwischen 80 und 90 Minuten. Es wurden zehn der 18 transkribierten Gruppendiskussionen ausgewählt und mittels qualitativer Inhaltsanalyse nach Mayring (2015) mit MAXQDA 2018 ausgewertet. Um das Material mit einer größtmöglichen Offenheit zu strukturieren, wurde ein induktives Vorgehen zur Kategorienbildung gewählt (Kuckartz 2014). Das Kategoriensystem wurde konsensuell validiert (Gläser \& Laudel 2010). Die Interkoderreliabilität betrug in allen Fällen mindestens $70 \%$.

\subsection{Wissensziele: das Wissensmanagement zweckorientiert betreiben}

Die Inhaltsanalyse ergab vier grundlegende Wissensziele im Handlungsfeld:

(1) Berufsorientierung gestalten: In allen Gruppendiskussionen bestand Konsens darüber, dass Lehrer*innen sowie weitere pädagogische Fachkräfte über ein grundlegendes Wissen zu den Maßnahmen und Handlungsoptionen in der Berufsorientierung verfügen müssen, um diese erfolgreich gestalten zu können. Kontrovers diskutiert wurde, welche Kenntnisse im Hinblick auf berufliche Perspektiven erforderlich seien: Es gab sowohl die Forderung, dass man mehr als nur eine Auswahl von Berufen kennen sollte, als auch das Argument, dass man nicht jedes Berufsbild kennen könne (BfA1d 193, EfK1d 48). Allerdings war sich die Mehrheit der Diskutanten darin einig, dass ein grundlegendes Verständnis über berufliche Möglichkeiten und Ausbildungswege unerlässlich sei und steter Weiterentwicklung bedürfe. Die Bedeutung von Einblicken in die Arbeitswelt jenseits der Institution Schule wurde thematisiert, um Schüler*innen auf die Arbeitswelt vorbereiten zu können (BfK1d 259).

Lehrer kennen häufig nur System Schule. Sie müssen bereit sein zu akzeptieren, dass es andere Systeme gibt und die so weit zu verstehen, wie sie es für ihre Schule brauchen. (AfN2w 161)

Um die Schüler*innen auf dem Weg zum Praktikum oder in die Ausbildung unterstützen zu können, sollten die pädagogischen Fachkräfte über Wissen zur 
Praktikums- oder Ausbildungsplatzsuche verfügen. Insbesondere seien hierbei Kenntnisse zum Umgang mit entsprechenden Online-Portalen unerlässlich (AmK1d 260, CfK1d 289, BfK1d 238).

Bezugnehmend auf berufliche Perspektiven wurden die Digitalisierung und deren Auswirkungen auf den Arbeitsmarkt diskutiert. Für die Beratung sei es heute wichtig, die Risiken der „Industrie 4.0“ zu kennen. Gleichzeitig erachten zwei Teilnehmende auch das Wissen über Chancen der Digitalisierung, etwa im Hinblick auf individuelle Unterstützungsmöglichkeiten für Schülerinnen und Schüler, als bedeutsam.

Die [gesellschaftliche Entwicklung] wird sich ja im- für diese jungen Menschen massiv verändern, [...] dass einige Berufe- Ihre Tätigkeit, die lernen=ein Handwerk, was demnächst=ein Roboter macht. Und da bin ich mir sehr sicher, dass sie, diese Schüler das erleben werden. Wie bereite ich sie in der Berufsorientierung darauf vor oder kann ich das überhaupt? (CfK1d 306)

Es gibt momentan so eine rasante Entwicklung, ich sag mal, (.) Technik 4.0, (.) wo man Schüler (2), oder, wo man Menschen mit, mit (.) körperlich motorischen Einschränkung, kognitiven Einschränkungen, so toll unterstützen könnte am Arbeitsplatz, ne. (.) Da gibt's ja vom, vom Buzzer bis hin zum Greifarm, den ich dann mit Augen steuere, (.) das ist ja=eine wahnsinnige Entwicklung, die da gerade voranschreitet. (DfN2w 25)

(2) Teilhabe für alle Schüler*innen ermöglichen: Die Gestaltung der Berufsorientierung im gemeinsamen Lernen könne nur auf Basis eines umfassenden Wissens im Handlungsfeld Inklusion gelingen. Dazu zählten profunde Kenntnisse zur Gestaltung von Teilhabe in der Schule und auf dem Arbeitsmarkt (BmA1w 7), ebenso eine umfassende sonderpädagogische Expertise (CfA2d 98). Neben dem Wissensaufbau auf personaler Ebene gelte es auch, Wissen auf organisationaler Ebene, etwa im Sinne von Leitlinien, zu schaffen und Einstellungen zum gemeinsamen Lernen in der Schulkultur zu etablieren.

(3) Unterstützung organisieren: In allen Gruppendiskussionen war man sich einig, dass keine pädagogische Fachkraft über ein allumfassendes Wissen im Feld der inklusiven Berufsorientierung verfügen könne. Vielmehr gehe es darum, ein Wissen über Kooperations- und Unterstützungsmöglichkeiten aufzubauen und sich einen Überblick über Zuständigkeiten und Kompetenzprofile anderer Professionen zu verschaffen (CmK3d 747, AfA2e 87).

Das ist genau das Wissen, was die brauchen, die müssen nicht alles (.) in die Tiefe. SIE MÜSSEN KEINE EXPERTEN SEIN, aber sie müssen, ich nenne das immer, bei denen muss an der richtigen Stelle zum richtigen Moment die Alarmglocke angehen, hier stimmt was nicht, wen frage ich jetzt. (AfA1w 138) 
(4) Individuell beraten: Im Hinblick auf eine individuelle Beratung von Schüler*innen wurden folgende Aspekte besonders häufig genannt und diskutiert: das Wissen über die Eigenschaften von Schüler*innen, über ihre Motivation und die soziale Herkunft. Zur gemeinsamen Eruierung beruflicher Möglichkeiten bedürfe es einer realistischen Einschätzung individueller Stärken, Schwächen (DfAld 184) und gegebenenfalls der Unterstützungsbedarfe $(\mathrm{CmN} 2 \mathrm{~d} 48)$. Als Voraussetzung für erfolgreiche Berufsorientierungsprozesse galt „einmal das Interesse für den Beruf und dann noch das weitere Interesse, was die Berufsorientierung et cetera bei (.) Schülern angeht" (CmK3d 384). In diesem Kontext wurde auch die Bedeutung der sozialen Herkunft thematisiert:

Und das macht mir Sorgen, wie kriege ich da die Schüler denn auch motiviert, wenn die Eltern sich schon weniger drum kümmern, wie (.) kriege ich die Schüler, ja, dahin, dass sie sich selber engagieren, weil das ja für ihre Zukunft ist, das (.) ist mir noch=ein Rätsel. (CfA1d 18)

\subsection{Wissensidentifikation: Wissensbestand und-defizite bilanzieren}

(1) Fehlendes internes Wissen identifizieren: Da in den Gruppen weitgehend problemorientiert diskutiert wurde, konnten vor allem Wissensdefizite ausgemacht werden. So seien Klassenleitungen mit der Berufsorientierung oft überfordert; es fehle Wissen zu den Maßnahmen und Handlungsoptionen, insbesondere im Kontext von Inklusion. Die standardisierten Maßnahmen des Landesprogramms „Kein Abschluss ohne Anschluss“ (KAoA) erforderten sehr viel „Know-how“ sowie ein starkes Maß an Eigeninitiative bei der Umsetzung und führten entsprechend oft zu Überforderungen (EfA2d 22). Eine Teilnehmerin konstatierte, ihr falle es schwer, einen Überblick über die verschiedenen beruflichen und schulischen Anschlussmöglichkeiten ihrer Schülerinnen und Schüler zu behalten (EfK3d 114). Konkret wurde benannt, dass vielen Lehrkräften das Wissen über Portale zur Berufsinformation sowie zur Praktikumsund Ausbildungsplatzsuche fehle.

Also ihr habt jetzt diese Anforderungen genannt, aber ich bin mir sicher, dass, wenn ich bei uns im Kollegium gucken würde, die Hälfte von den Kollegen wüssten selber nicht, wo die nachschauen müssten. Auch welche Portale es im Internet gibt, ob Stepstone, Monster oder Indeed, wo man jetzt mittlerweile Jobs finden kann, Praktika [...]. (AmK1d 260)

(2) Experten(wissen) identifizieren: Das Handlungsfeld der inklusiven Berufsorientierung umfasse Wissen, das sich ständig verändert, [sodass] es eine völlig illusorische Vorstellung ist, dass ein Lehrer das gewissermaßen erlernen und sichern kann“ (AmA1w 7). Daher solle es weniger darum gehen, jeder Lehrkraft ein allumfassendes Wissen zu den Themen Inklusion und Berufsorientierung $\mathrm{zu}$ vermitteln, als vielmehr darum, ein Bewusstsein für die 
Bedeutung einer multiprofessionellen Zusammenarbeit zu schaffen: Es gelte, relevantes Expertenwissen und die entsprechenden Wissensträger zu identifizieren. Als außerschulische Kooperationspartner, die als Wissensquelle essenziell seien, wurden genannt: Betriebe, die Agentur für Arbeit, der Integrationsfachdienst, die Kammern sowie die Landschaftsverbände. ${ }^{1}$

Innerhalb der Institution Schule konnten ebenfalls Träger unterschiedlichen Wissens identifiziert werden: Die Klassenleitungen verfügten im Regelfall über ein grundlegendes Wissen über ihre Schülerschaft im Allgemeinen, dagegen wurde sonderpädagogischen Fachkräften im gemeinsamen Lernen ein Expertenwissen im Hinblick auf die Eigenschaften und Bedarfe der Schülerinnen und Schüler mit sonderpädagogischem Förderbedarf zugesprochen. Dementsprechend wurde die innerschulische Zusammenarbeit als Voraussetzung einer ,guten Inklusion“ beschrieben:

Schulen, die gute Inklusion machen, die zeichnen sich dadurch aus, dass die gute, intern Arbeitsstrukturen entwickeln und zusammen Unterricht planen oder (.) diagnostische Prozesse begleiten. Und (.), ich sag mal [...] zusammen an dem Thema arbeiten, (.) also das muss=eine Schule leisten und das müssten die Kollegen haben können, ne, dass sie, also- Und man muss ja nicht alles wissen, so wie er, der (.) in vielen Jahren Wissen (.) in dem Bereich angehäuft hat, aber diese Fähigkeit und die Bereitschaft, dieses sich zusammen zu erarbeiten, ne. Die Intelligenz des Schwarms. (BmN2w 178)

Als bedeutsame Wissensträger gelten die Koordinatorinnen und Koordinatoren für Studien- und Berufsorientierung $\left(\right.$ StuBos) ${ }^{2}$ (unter anderem EfA1d 6, EFA2d 22, BfA2d 75). Dabei wurde deren Rolle klar definiert und eingegrenzt:

Und der StuBo ist in so großen Systemen oder das StuBo-Team ja eher, die Organisatoren, die Sachen initiieren, Konzepte erstellen dann entsprechend auch und die Kommunikation zu den Lehrkräften übernehmen. Aber die, die Verantwortung liegt auch bei der Klassenleitung und bei jedem Fachlehrer, das- gibt es ja auch Möglichkeiten, dass man (.) das fachbezogen aufteilt nochmal. (AfN2d 96)

Es wurde allerdings konstatiert, dass die Schulen diesem Anspruch nicht immer gerecht würden:

1 Bei den Landschaftsverbänden handelt es sich um bundeslandspezifische Organisationen, die in Nordrhein-Westfalen z. B. als Träger des Inklusionsamtes für die Teilhabe schwerbehinderter Menschen und ihnen gleichgestellter auf dem allgemeinen Arbeitsmarkt zuständig sind und entsprechende Unterstützung leisten (Landschaftsverband Rheinland 2019).

2 Die Koordinatorinnen und Koordinatoren für die Berufliche Orientierung werden in Nordrhein-Westfalen an allen weiterführenden Schulen zur Initiierung und Umsetzung von Studien- und Berufswahlprozessen eingesetzt. Sie sind von den Schulleitungen zu benennen (Ministerium für Schule und Bildung 2019, Kap. 1). 
Also die [...] Klassenlehrer, die haben damit gar nicht am Hut, die wissen (.) in der 9. Klasse gibt's=ein 3-Wochen-Praktikum und in der 8. Klasse drei Tage. Dann kümmern die sich=ein bisschen da drum, acht, in der 7. Klasse sind Potenzialanalyse, da fahren die nach Essen, zweimal die Woche. [...] Das ist das einzige, was die-, womit sie sich beschäftigen. Aber auch weil sie es nicht so richtig gele- also, ne, gelernt haben. (AmA1d 84)

(3) Transparenz schaffen: Die Problematik einer fehlenden Transparenz über den Wissensstand wurde sowohl im Hinblick auf Prozesse innerhalb einer Schule als auch den Wissenstransfer zwischen Schulen thematisiert. Zunächst gelte es, innerorganisationale Transparenz über Handlungsoptionen und Maßnahmen zu schaffen sowie Erfahrungswerte mit dem Kollegium zu teilen (AfA2d 54). Darüber hinaus müsse es einen Austausch zwischen Schulen geben, um vorhandene Wissensbestände zu nutzen, zum Beispiel müsse es „Konferenzen zwischen abgebenden und der übernehmenden Schule (.) geben [...] zu den Schülern, die da ankommen" (AfA2e 102), jedoch verhindere auch das Datenschutzgesetz eine stärkere Zusammenarbeit.

\subsection{Wissenserwerb: personales und organisationales Wissen auf-/ausbauen}

In den Gruppen herrschte Konsens darüber, dass die Themen Inklusion und Berufsorientierung in der ersten und zweiten Phase der Lehrerbildung nicht oder nur unzureichend thematisiert würden (DfA1d 51, AfA1w 15, EfA3e 224), ebenso in der Fortbildung: Zwar gebe es Fortbildungen sowohl zur Inklusion als auch zur Berufsorientierung, es fehle jedoch an Veranstaltungen, die beides miteinander verbinden (EfA1d 46, CfA3e 64). Dabei fielen auch die Bewertungen zu den Fortbildungsinhalten kritisch aus.

Ja, wir basteln da immer rum. Wir haben Fortbildungen besucht, aber, ich meine, so=eine eintägige Fortbildung oder so, (.) ne. @(2)@, sagt- hat man keine Ahnung danach. (BfA2e 68)

Einen Großteil des Wissens über die inklusive Berufsorientierung würden die pädagogischen Fachkräfte eigenständig entwickeln. Dies erfordere ,sehr viel persönliches Engagement und sehr viel Zeit“" (AfA2d 54). Zudem wurde die Bedeutung des Wissenserwerbs von externen Wissensträgern verdeutlicht:

Wir haben jetzt gerade geschafft, dass [der] Integrationsfachdienst, der bisher anders aufgestellt war, eine Kollegin hat, die macht nichts anderes als GL, die reist also den ganzen Tag durch [Stadt in NRW] und (.) klappert die Schulen ab. Die hat vor den Sommerferien (.) alle Lehrer, die da tätig waren, kennengelernt, hatte ihnen genau diese komplizierten Unterschiede zwischen STAR, GL, KAoA, Regel und all was wir da haben, erklärt. (AfA1w 46) 
Neben dem eigenständigen Wissenserwerb spielt also auch der Einbezug von Expertenwissen eine Rolle. So werde etwa das Wissen von Integrationshelfern und Job-Coaches vor allem durch ihre direkte Beteiligung an Beratungsprozessen genutzt (BmN2d 166). Bei der Berufsorientierung im gemeinsamen Lernen sei die Expertise sonderpädagogischer Fachkräfte essenziell, doch der Wissenstransfer von der Förder- zur Regelschullehrkraft und umgekehrt gestalte sich mitunter schwierig. Ein Sonderpädagoge mahnte an, dass die Unterstützung von Regelschullehrkräften angenommen werden müsse (DmK3d 458). Ähnlich hielt eine Diskutantin fest:

Und das dritte ist nun, also ich nenne es jetzt mal, so ein bisschen Bewusstseinsebene vom Lehrer. Also es gibt ja immer (.) den alten Spruch, ne, "my class is my castle" und das muss weg, ne. "My class ist not my castle." Ich muss ganz viele reinholen, weil alleine ist das völlig unmöglich. (AfA1w 23)

\section{Implikationen für die Praxis - Desiderate der Forschung}

Aus diesen Ergebnissen zum Realitätsausschnitt der inklusiven Berufsorientierung lassen sich Qualifizierungsbedarfe der pädagogischen Fachkräfte und Entwicklungsbedarfe der Schulen sowie Forschungsdesiderate ableiten.

\section{Lehrerbildung, insbesondere Studium und Fortbildung}

Es sind Wissensdefizite betreffend die Handlungsoptionen und Maßnahmen der Berufsorientierung sowie die schulischen und beruflichen Anschlusswege zu konstatieren. Da diese Wissenselemente als wesentliche Wissensziele ausgewiesen wurden, kann daraus ein konkreter Qualifizierungsbedarf gefolgert werden. Zudem scheint der Wissenserwerb im Kontext von Inklusion durch Regelschullehrkräfte weitgehend ungesteuert zu erfolgen. Aus den Daten wird ersichtlich, dass die Vermittlung entsprechender Inhalte in allen drei Phasen der Lehrerbildung gewünscht wird. Die 2016 neugefasste Lehramtszugangsverordnung schreibt inzwischen inklusionsbezogene Studienanteile von mindestens 14 Leistungspunkten verbindlich vor, so dass zukünftige Lehrerinnen und Lehrer über eine bessere Ausgangsbasis verfügen dürften. Und sie eröffnet - jedoch nur im Lehramt für Haupt-, Real-, Sekundar- und Gesamtschulen die Option, einen Profilbereich „Berufswahl/Berufsorientierung“ einzurichten. Von letzterem macht allerdings landesweit keine Universität Gebrauch; doch selbst wenn, so blieben beide Bereiche - Inklusion und Berufsorientierung im Studium gleichwohl systematisch unverbunden. Für die zielgerichtete Fortund Weiterbildung der pädagogischen Fachkräfte sollten Formate entwickelt werden, die die bislang auch dort separierten Gebiete verbinden. 
Die Lehrkräfte betonten die Bedeutung eigener, außerschulischer Berufserfahrungen. Mit dem seit 2009 für alle lehramtsqualifizierenden Studiengänge obligatorischen, mindestens vierwöchigen „Berufsfeldpraktikum“, das seit der Reform des nordrhein-westfälischen Lehrerausbildungsgesetzes von 2016 ,in der Regel“" außerschulisch sein soll (§ 12 Abs. 1 Nr. 2), wurde ein „Praxiselement" geschaffen, das Einblicke in die Wirtschaft und Arbeitswelt ermöglicht. Lehrerbetriebspraktika, die im nordrhein-westfälischen Runderlass zur beruflichen Orientierung unter Nr. 7 „Qualifizierung der Lehrkräfte“ recht ausführlich dargelegt werden, bieten weitere Möglichkeiten der Sammlung einschlägiger Erfahrungen. Sie zu organisieren obliegt der Eigenverantwortung der Schulen; sie zu genehmigen der Schulleitung, was ihr allerdings aktuell recht schwerfallen dürfte, da sie zwei bis vier Wochen dauern sollen, vielfach jedoch akuter Lehrermangel herrscht und gleichzeitig die Minimierung von Unterrichtsausfall gefordert ist - ein Zielkonflikt für die Schulleitungen.

Wie bereits bei der Erörterung des Modells für das Wissensmanagement von Probst, Raub und Romhardt (2012), so lässt auch das empirische Material die Verbindungen zwischen den Wissensbausteinen erkennen. So ist etwa die Reflexion der eigenen Wissensgrenzen im Baustein „Wissensziel“ eine Voraussetzung für den Baustein „Wissenserwerb“, in Form des Einbezuges von weiterem Expertenwissen, das in einem direkten Prozess der Zusammenarbeit erlangt werden kann. Es bedarf dazu der Bereitschaft, Erfahrungswerte mit Kolleginnen und Kollegen zu teilen. Insofern bestätigen die Ergebnisse jenen Ausschnitt aus dem Referenzrahmen - wenn auch nur auf Basis qualitativer Daten und nicht mit Anspruch auf Allgemeingültigkeit.

Dem Baustein „Wissenserwerb“ zuzuordnende Passagen verdeutlichen die tragende Rolle personaler Fähigkeiten beim Aufbau eines schulischen Wissensmanagements. Die Bereitschaft Wissen anzunehmen wurde insbesondere in Fällen der gemeinsamen Beratung von Schüler*innen mit besonderem Unterstützungsbedarf gefordert. Bezüglich der individuellen Einflussfaktoren der Schülerinnen und Schüler erscheint es notwendig, ein Bewusstsein dafür zu schaffen und entsprechendes Wissen aufzubauen, denn beim gemeinsamen Lernen sollten alle Schüler*innen individuell betrachtet werden und nicht bestimmte Gruppen vorrangig. Das „Risiko curricularer und organisatorischer Fehlsteuerung“ (Baethge 2014, 230) kann nur so vermieden werden. Als ein relevanter Einflussfaktor sei hier nur die soziale Herkunft genannt, deren besondere Relevanz im Übergangssystem von Brändle und Müller (2014) thematisiert wird.

Die Analyse von Wissenszielen bekräftigte die Relevanz personaler Fähigkeiten insofern, als die Notwendigkeit der Selbstreflexionsfähigkeit betont wurde. Sich der eigenen Grenzen des Wissensaufbaus bewusst zu sein, geht dem Eruieren von Kooperations- und Unterstützungsmöglichkeiten voraus. Zu ähnlichen Schlussfolgerungen gelangt Meschenmoser (2001) im 
Kontext von Wissensmanagement in der schulischen Allgemeinbildung. Insbesondere in Arbeitsbereichen, in denen die beteiligten pädagogischen Fachkräfte unterschiedliche Wissensbereiche abdecken, ist ihre Zusammenarbeit unerlässlich. Dies erfordert zunächst die Identifikation von inner- und außerschulischen Wissensträgern. Diesbezüglich bedarf es zudem weiterer Untersuchungen zu den Aufgabenbereichen und Zuständigkeiten der Akteure.

Schulentwicklung, insbesondere organisationales Wissensmanagement

Auch zehn Jahre nach der Ratifizierung der UN-Behindertenrechtskonvention ist die Inklusion ein aktuelles Schulentwicklungsthema, nicht zuletzt im Handlungsfeld der inklusiven Berufsorientierung. Hier bieten Driesel-Lange, Hany und Kracke et al. (2011) Anknüpfungsmöglichkeiten an die von ihnen entwickelten Qualitätskriterien einer berufswahlkompetenten Schule, die sich auf Ebene des Personals, des Unterrichts sowie der Schulorganisation verorten lassen. Besondere Bedeutung kommt dabei etwa der Entwicklung eines Schulkonzeptes sowie der Befähigung zur Selbstreflexion im Hinblick auf Entwicklungs- und Fortbildungsbedarfe zu. Auf Basis unserer Untersuchung lassen sich insbesondere aus der festgestellten fehlenden Transparenz von Handlungsoptionen und schulischen Wissensständen auf der Ebene der Schulorganisation Entwicklungsbedarfe ableiten. In diesem Zusammenhang wird organisationalem Wissensmanagement attestiert, einen wichtigen Beitrag zu Schulentwicklungsprozessen leisten zu können (Heitmann 2013). Als Ansatzpunkte für die Praxis nennt sie die „Erleichterung der Zugänglichkeit personalen Wissens“ sowie die „Organisierung des Austauschs im Kollegium“ (ebd., 304). Auf Basis der Analyse der drei Wissensbausteine lassen sich schulische Entwicklungsbedarfe zusammenfassen, denen dabei Rechnung zu tragen wäre.

Das Wissen über Kooperations- und Unterstützungsmöglichkeiten wurde bei der Untersuchung als eines von vier Wissenszielen ausgemacht. Dabei wurde festgehalten, dass eine Zusammenarbeit nur dann möglich ist, wenn Zuständigkeiten und Kompetenzprofile anderer Professionen bekannt sind. Organisationales Wissensmanagement muss dementsprechend nicht nur Sorge für die Identifikation der Wissensträger tragen, sondern auch die Sichtbarkeit personalen Wissens erhöhen, etwa durch „Wissensträgerkarten“ (ebd., 286). Die dadurch mögliche koordinierte Zusammenarbeit der an der inklusiven Berufsorientierung beteiligten Akteure erfordert nicht zuletzt soziale Kompetenzen sowie Kooperationsbereitschaft. Dafür sei das Aufstellen von Regeln einer guten Zusammenarbeit förderlich. Insbesondere einer gegenseitigen Wertschätzung der Expertise anderer Berufsgruppen kommt an dieser Stelle eine große Bedeutung zu. Die Bereitschaft, unter Kolleginnen und Kollegen Erfahrungswerte zu teilen, schafft ferner die Basis für eine transparente Kommunikation im System Schule. Ein gezielter Austausch im Kollegium kann darüber hinaus über die Etablierung „kollektiver Austauschforen“ 
erfolgen, in denen zum Beispiel über Veränderungen gesetzlicher Vorgaben zur Berufsorientierung sowie innerschulische (Weiter-)Entwicklungen informiert wird (Heitmann 2013). Dieser Kooperations- und Kommunikationsauftrag erfordert schließlich eine gezielte Vorbereitung der angehenden Lehrkräfte sowie der weiteren pädagogischen Fachkräfte. So fordern Hopmann, Böhm-Kasper und Lütje-Klose (2019) insbesondere im Hinblick auf inklusive Settings, einen Lernbereich zum Thema multiprofessionelle Kooperation in der universitären Lehrerbildung zu verankern.

Zusammenfassend zeichnen sich in der dichten Beschreibung der Wissensbausteine im Feld der inklusiven Berufsorientierung drei Kompetenzfacetten als Anknüpfungspunkte für Bildungsformate ab: affektiv-motivational, sozial-kommunikativ und metakognitiv. Da diese Ergebnisse jedoch bislang lediglich im Rahmen eines qualitativen Verfahrens erzielt wurden, tragen sie den Status von Hypothesen und könnten noch nicht als Vorlage für bundeslandübergreifende Aus- und Weiterbildungsanstrengungen für Lehrer*innen sowie weitere pädagogische Fachkräfte dienen. Hier wäre mit dem Instrumentarium quantitativer Forschung zunächst zu versuchen, die vorliegenden Befunde zu sichern - oder eben zu falsifizieren und auf diese Weise ein dann anscheinend bundeslandspezifisches Qualifizierungserfordernis unter erneuter Hypothesenbildung zu präzisieren und ggf. zu generalisieren.

Eine Frage, deren Beantwortung weitergehender Forschung bedarf, ist, ob die Wissensbausteine eine unterschiedliche Relevanz für unterschiedliche Professionen und/oder Funktionen in der Schule besitzen. Die Schulleitung dürfte mutmaßlich andere Kompetenzen im Bereich des Wissensmanagements benötigen, als Studien- und Berufsorientierungskoordinatoren, und evtl. werden für die inklusive Berufsorientierung solche Wissensziele von Regelschullehrkräften gesetzt, wie sie bislang von Sonderpädagoginnen und -pädagogen oder Sozialarbeiterinnen und -arbeitern verlangt wurden. Lassen sich entsprechende Hypothesen quantitativ sichern, so könnten sie die Basis für differenzierte Ausund Weiterbildung pädagogischer Fachkräfte liefern.

Im Hinblick auf die Schulentwicklung konnte zudem vor allem die Bedeutung der Transparenz und Identifikation von Wissensträgern sowie der koordinierten Zusammenarbeit aufgezeigt werden. Dabei gilt es anzumerken, dass die Ergebnisse in Bezug zu den gesetzlichen Vorgaben in NordrheinWestfalen stehen und aufgrund des föderalen Bildungssystems, das sich auch in den länderspezifischen Vorgaben zur Berufsorientierung widerspiegelt, in anderen Bundesländern anders ausfallen könnten. Neben der Frage nach einer Generalisierbarkeit dieser Ergebnisse sollten auch die inner- und außerschulischen Akteure in der inklusiven Berufsorientierung als Wissensträger näher untersucht werden. 


\section{Literatur}

Bach, A., Schmidt, C. \& Schaub, C. (2016). Professionalisierung von Lehrkräften für eine inklusive gewerblich-technische Berufsbildung. bwp@ Berufs- und Wirtschaftspädagogik - online, 30, 1-24. http://www.bwpat.de/ausgabe30/bach_ schmidt_schaub_bwpat30.pdf (02.06.2020).

Baethge, M. (2014). Der vergessene Bildungsraum: Übergang von der allgemeinbildenden Schule in Ausbildungs- und Arbeitsmarkt. Unterrichtswissenschaft, 42(3), 224-243.

Baumert, J. (1998). Fachbezogenes-fächerübergreifendes Lernen - Erweiterte Lernund Denkstrategien. In Bayerisches Staatsministerium für Unterricht, Kultus, Wissenschaft und Kunst (Hrsg.), Wissen und Werte für die Welt von morgen (S. 214231). München.

Baumert, J. \& Kunter, M. (2006). Stichwort: Professionelle Kompetenz von Lehrkräften. Zeitschrift für Erziehungswissenschaft, 9(4), 469-520.

Bergs, L. \& Niehaus, M. (2016). Berufliche Bildung. In I. Hedderich, G. Biewer, J. Hollenweger \& R. Markowetz (Hrsg.), Handbuch Inklusion und Sonderpädagogik (S. 293-297). Bad Heilbrunn: Klinkhardt.

Bienengräber, T., Retzmann, T., Greiten, S. \& Turhan, L. (2019). Berufsorientierung im Gemeinsamen Lernen der Sekundarstufen - Skizze eines Forschungsprojekts. In M. Esefeld, K. Müller \& P. Hackstein et al. (Hrsg.), Inklusion im Spannungsfeld von Normalität und Diversität (S. 103-110). Bad Heilbrunn: Klinkhardt.

Blömeke, S., Kaiser, G. \& Lehmann, R. (2008). Professionelle Kompetenz angehender Lehrerinnen und Lehrer: Wissen, Überzeugungen und Lerngelegenheiten deutscher Mathematikstudierender und -referendare. Erste Ergebnisse zur Wirksamkeit der Lehrerausbildung. Münster: Waxmann.

Brändle, T. \& Müller, S. (2014). Berufsorientierung von Benachteiligten Jugendlichen. Sozialer Fortschritt, 63(4/5), 82-89.

Brüggemann, T., Driesel-Lange, K. \& Weyer, C. (2017). Instrumente zur Berufsorientierung. Pädagogische Praxis im wissenschaftlichen Diskurs. Münster: Waxmann.

Buchmann, U. \& Bylinski, U. (2013). Ausbildung und Professionalisierung von Fachkräften für eine inklusive Berufsbildung. In H. Döbert \& H. Weishaupt (Hrsg.), Inklusive Bildung professionell gestalten. Situationsanalyse und Handlungsempfehlungen (S. 147-202). Münster: Waxmann.

Chott, P. O. (1998). Das Lehren des Lernens. PÄDForum, 2(26/11), 174-180.

Davenport, Th. H. \& Prusak, L. (2000). Working knowledge: How organizations manage what they know. Boston, MA: Harvard Business School Press.

Dick, W. \& Wehner, T. (2002). Wissensmanagement zur Einführung: Bedeutung, Definition, Konzepte. In W. Lüthy, E. Voit \& Th. Wehner (Hrsg.), Wissensmanagement-Praxis. Einführung, Handlungsfelder und Fallbeispiele (S. 7-27). Zürich: vdf.

Dreer, B. (2013). Kompetenzen von Lehrpersonen im Bereich der Berufsorientierung. Wiesbaden: Springer VS.

Driesel-Lange, K., Hany, E., Kracke, B. \& Schindler, N. (2011). Konzepte und Qualitätsmerkmale schulischer Berufsorientierung an allgemeinbildenden Schulen. DDS, 4(3), 312-325. 
Driesel-Lange, K., Kracke, B., Hany, E. \& Schindler, N. (2013). Das Thüringer Berufsorientierungsmodell: Charakteristika und Bewährung. In T. Brüggemann, S. Rahn (Hrsg.), Berufsorientierung. Ein Lehr- und Arbeitsbuch (S. 281-297). Münster: Waxmann.

Gläser, J. \& Laudel, G. (2010). Experteninterviews und Qualitative Inhaltsanalyse als Instrumente Rekonstruierender Untersuchungen (4. Aufl.). Wiesbaden: VS Verlag.

Greiten, S., Bienengräber, T., Retzmann, T., Turhan, L. \& Schröder, M. (2020). Kompetenzen von Lehrkräften und weiteren pädagogischen Fachkräften als Gelingensbedingungen der inklusiven Berufsorientierung in allgemein- und berufsbildenden Schulen am Beispiel des Organisierens von Schülerbetriebspraktika. Journal für Psychologie, 2(27), 313-335.

Hamm, C. (2004). Schulentwicklung und Bildungsnetzwerke-Eine wirtschaftspädagogische Modellierung der für den Aufbau und die Nutzung von Bildungsnetzwerken förderlichen Bedingungen in berufsbildenden Schulen. Dissertation, Universität zu Köln.

Heitmann, K. (2013). Wissensmanagement in der Schulentwicklung - theoretische Analyse und empirische Exploration aus systemischer Sicht. Wiesbaden: Springer VS.

Hinz, A. (2002). Von der Integration zur Inklusion - terminologisches Spiel oder konzeptionelle Weiterentwicklung? Zeitschrift für Heilpädagogik, 53(9), 354-361.

Hinz, A. (2009). Inklusive Pädagogik in der Schule. Zeitschrift für Heilpädagogik, 60(5), 171-179.

Hopmann, B., Böhm-Kasper, O. \& Lütje-Klose, B. (2019). Multiprofessionelle Kooperation in inklusiven Ganztagsschulen in der universitären Lehre. Entwicklung inklusions- und kooperationsbezogener Einstellungen von angehenden Lehrkräften und sozialpädagogischen Fachkräften in einem interdisziplinären Masterseminar. Herausforderung Lehrerinnenbildung, 2(3), 400-421.

Huber, S. G. \& Krey, J. (2009). Wissensmanagement in Teams. In S. G. Huber (Hrsg.), Handbuch für Steuergruppen. Grundlagen für die Arbeit in zentralen Handlungsfeldern des Schulmanagements (S. 327-340). Köln: Link-Luchterhand.

Koch, B. (2015). Berufsorientierung in einer inklusiven Schule.bwp@Berufs-und Wirtschaftspädagogik - online, 27, 1-18. http://www.bwpat.de/ausgabe27/ koch_bwpat27.pdf (02.06.2020).

Kuckartz, U. (2014). Mixed Methods: Methodologie, Forschungsdesigns und Analyseverfahren. Wiesbaden: Springer Fachmedien.

Landschaftsverband Rheinland (2019). Online: https://www.lvr.de/de/nav_main/soziales_1/inklusionsamt/wir_ueber_uns/das_lvr_integrationsamt/integrationsamt.jsp, (12.12.2019).

Lamnek, S. (2005). Gruppendiskussion: Theorie und Praxis (2. Aufl.). Weinheim u. a.: Beltz.

Lazarides, R., Ohlemann, S., Wills, R., Henze, U. \& Ittel, A. (2015). Die Perspektive von Lehrerinnen und Lehrern auf schulische Berufs- und Studienorientierung in der Sekundarstufe. Schulpädagogik heute, 6(12), 3-16.

Löser, J. M. \& Werning, R. (2015). Inklusion - allgegenwärtig, kontrovers, diffus? Erziehungswissenschaft, 26(51), 17-24.

Mayhack, K. \& Kracke, B. (2010). Unterstützung der beruflichen Entwicklung Jugendlicher: Der Beitrag von Lehrer/innen und Eltern. Diskurs Kindheits- und Jugendforschung, 4, 397-411. 
Mayring, P. (2015). Qualitative Inhaltsanalyse: Grundlagen und Techniken (12. Auf1.). Basel: Beltz.

Meschenmoser, H. (2001). Wissensmanagement in der schulischen Allgemeinbildung/ Chancen und Konsequenzen (S. 133-143). Conference: Informatikunterricht und Medienbildung, INFOS 2001, 9. GI-Fachtagung Informatik und Schule, 17.-20. September 2001 in Paderborn.

Ministerium für Schule und Bildung (2019). Berufliche Orientierung (Ausbildungsund Studienorientierung). RdErl. d. Ministeriums für Schule und Bildung v. 16.09.2019 (ABl. NRW. 11/19) Düsseldorf.

Moser, V. (2016). Professionelle Kooperation in inklusiven Schulen aus sonderpädagogischer Perspektive. In A. Kreis, J. Wick \& C. Kosorok Labhart (Hrsg.), Kooperation im Kontext schulischer Heterogenität (S. 159-169). Münster: Waxmann.

Nentwig, L. (2018). Berufsorientierung als unbeliebte Zusatzaufgabe in der Inklusion? Eine Studie zur Bedeutsamkeit der professionellen Handlungskompetenz unter Fokussierung der motivationalen, volitionalen und sozialen Bereitschaften von Lehrpersonen zum Engagement in der inklusiven Berufsorientierung. Dissertation, Technische Universität Dortmund.

Pawlowsky, P. (1994). Wissensmanagement in der lernenden Organisation. Paderborn.

Probst, G. J. B. \& Büchel, B. S. T. (1994). Organisationales Lernen. Wettbewerbsvorteil der Zukunft. Wiesbaden: VS Verlag.

Probst, G. J. B., Raub, S. \& Romhardt, K. (2012). Wissen managen: wie Unternehmen ihre wertvollste Ressource optimal nutzen. Wiesbaden: VS Verlag

Rahn, S., Brüggemann, T. \& Hartkopf, E. (2013). Berufliche Orientierungsprozesse Jugendlicher in der Sekundarstufe I. Ergebnisse aus dem Berufsorientierungspanel (BOP). In T. Brüggemann \& S. Rahn (Hrsg.), Berufsorientierung. Ein Lehr- und Arbeitsbuch (S. 141-156). Münster: Waxmann.

Reinmann-Rothmeier, G. (2001). Wissen managen. Das Münchener Modell. Forschungsberichte der LMU 131. München.

Sander, A. (2004). Konzepte einer Inklusiven Pädagogik. Zeitschrift für Heilpädagogik, $5,240-244$.

Sarnitz, A. (2011). Einflussfaktoren auf den Wissenstransfer in Projektübergabeprozessen und Handlungsoptionen von Optimierungen von Projektübergaben. Dissertation, Universität Stuttgart.

Schüppel, J. (1996). Wissensmanagement. Organisatorisches Lernen im Spannungsfeld von Wissens- und Lernbarrieren. Wiesbaden: Deutscher Universitäts-Verlag.

Weinert, F. E. (2001). Vergleichende Leistungsmessung in Schulen - eine umstrittene Selbstverständlichkeit. In F. E. Weinert (Hrsg.), Leistungsmessungen in Schulen (S. 17-31). Weinheim: Beltz.

Wiater, W. (2007). Wissensmanagement. Eine Einführung für Pädagogen. Wiesbaden: VS Verlag für Sozialwissenschaften.

Willke, H. (2001). Systemisches Wissensmanagement (2. Aufl.). Stuttgart: Lucius und Lucius. 


\title{
Berufsdarstellungen in Vorabendserien zwischen 1990 und 2018 als Informationsquellen über Berufe
}

\author{
Heike Jahncke, Florian Berding, Jane Porath und Christian Steib
}

\section{Einleitung}

Auch wenn es heute nicht mehr für alle Menschen gleichermaßen gilt, dass sie ihr ganzes Leben in dem zu Beginn ihres Berufslebens erlernten Beruf arbeiten müssen und werden, ist die Wahl des Berufs für einen jungen Menschen eine weitreichende und bedeutende Entscheidung, weil sie dessen Arbeits- und Lebenschancen und -risiken und dessen berufliche und allgemeine Zufriedenheit zu einem großen Teil festlegt (Gehrau 2014). Jedoch ist diese Entscheidung für die jungen Menschen oftmals mit einer großen Unsicherheit behaftet, da sie regelmäßig bereits während der Schulzeit getroffen werden muss, also zu einem Zeitpunkt, zu dem die jungen Menschen selbst nur wenige Informationen über Berufe und Arbeit sammeln konnten (ebd.). Genau hier setzt die Berufsorientierung an: ,Das Ziel der pädagogischen Unterstützung der Berufsorientierung ist es also, den gesamten Berufsorientierungsprozess so zu fördern, dass die Wahrscheinlichkeit steigt, dass die Jugendlichen die einzelnen Teilaufgaben des Berufswahlprozesses [...] vollständig und vor allem rechtzeitig bearbeiten und lösen“ (Brüggemann \& Rahn 2013, 16).

Die Entwicklungsaufgabe der Berufswahl stellt sich Jugendlichen in der Regel in einem Alter zwischen dem 14. und 24. Lebensjahr (Blickle 2014). Die dazu notwendige Fähigkeit lässt sich als Berufswahlreife bzw. als Berufswahlkompetenz umschreiben. Die Berufswahlreife meint sowohl die Fähigkeit als auch die Bereitschaft, mit den spezifischen Entwicklungsaufgaben im Berufswahlprozess erfolgreich umgehen zu können und darüber hinaus die Realisierung eines bestimmten Berufes anzustreben (Hartkopf 2013). Die Berufswahlkompetenz kann verstanden werden als ,die Bereitschaft und Fähigkeit, die in bestimmten beruflichen Entwicklungsphasen gestellten Berufswahlaufgaben wahrzunehmen, ihren Problemgehalt zu analysieren, dabei die in ihnen liegenden Chancen zur Selbstbestimmung zu entdecken und zu Handlungsmöglichkeiten auszuarbeiten, diese zu entscheiden und mit persönlichen sowie sozialen Bindungsfolgen zu versehen, d. h. zu verantworten, und sie in ein situationsgerechtes Verwirklichungshandeln einzubringen“(Bußhoff 1984, 67). Die Berufswahlkompetenz bzw. die Berufswahlreife beinhalten als Dimensionen neben der Berufswahlzuversicht, Planungskompetenz, Entscheidungskompetenz 
und Wissen über das eigene Selbst insbesondere auch Wissen über die relevante berufliche Umwelt (Blickle 2014).

Neben dem familiären Umfeld können vor allem Medien als eine Informationsquelle über Berufe dienen. Die aktuelle JIM-Studie zeigt dabei, dass mittlerweile mindestens 93\% der Jugendlichen über ein Handy bzw. ein Smartphone verfügen, aber nur rund $65 \%$ über einen Computer bzw. einen Laptop und nur 50\% über ein Fernsehgerät (Feierabend, Rathgeb \& Reutter 2020, 7). Das Fernsehen nimmt bei der Beschäftigung mit Medien im Jahr 2019 dementsprechend den fünften Platz hinter Internet, Smartphone, Musik hören und Online-Videos ein, während online Video-Streaming-Dienste den achten und die Betrachtung von aufgezeichneten Serien, DVDs und Blurays den elften Platz erreichen (ebd.). So sehen rund 76\% der Jugendlichen täglich oder mehrmals die Woche TV, während 84\% der Jugendlichen mehrmals die Woche oder täglich Online-Videos betrachten. Anders war die Situation noch im Jahr 1998. Hier kommt die JIM-Studie der damaligen Zeit zu dem Ergebnis, dass rund 95\% der Jugendlichen täglich oder mehrmals die Woche fernsehen (Medienpädagogischer Forschungsverbund Südwest 2018). Die Entwicklung im Zeitablauf verdeutlicht Abbildung 1.

Abb. 1: Entwicklung der Fernseh- bzw. Fernsehnahen Nutzung von Medien durch Jugendliche auf der Grundlage der JIM-Studien von 1998 bis 2019

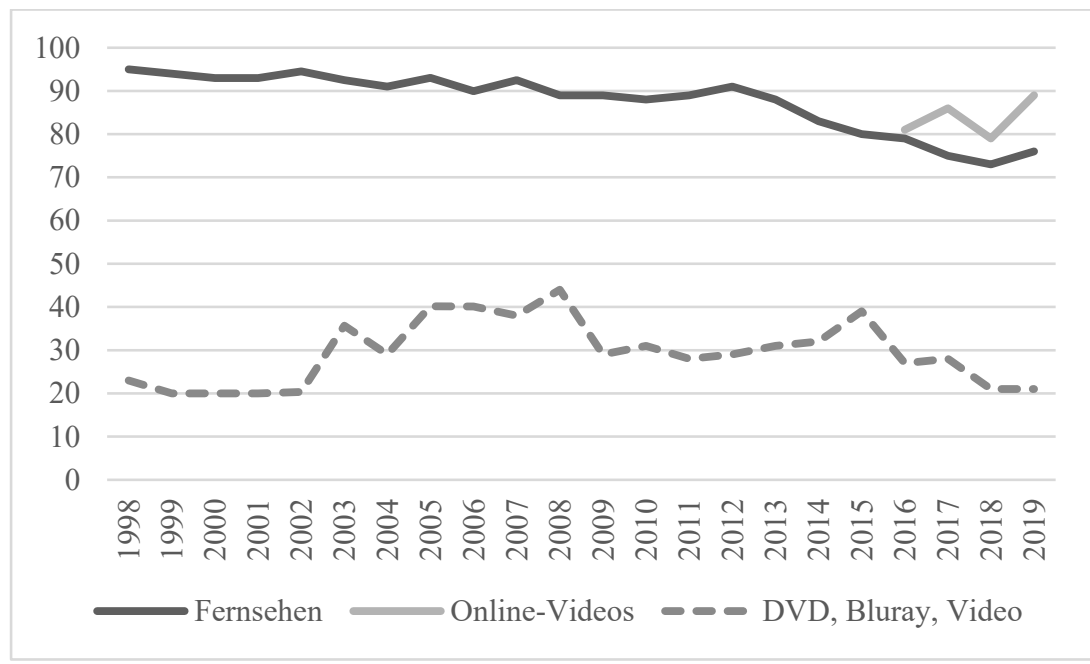

Quelle: eigene Darstellung

Allerdings zeigt die aktuellste vorliegende KIM-Studie für Kinder zwischen 6 und 13 Jahren, dass rund 65\% der Mädchen und 63\% der Jungen Vorbilder 
besitzen und diese vor allem aus dem TV bzw. Filmen stammen (Feierabend, Plankenhorn \& Rathgeb 2017). Deutlich wird damit, dass das klassische Fernsehen aktuell nicht mehr das dominante Medium der Jugendlichen ist, gleichwohl aber immer noch eine mittlere bis hohe Bedeutung besitzt.

Entsprechend der nach wie vor hohen Bedeutung des Fernsehens, liegen einige Studien vor, die untersuchen, ob der Konsum von Fernsehserien die Berufswahlentscheidungen von Jugendlichen beeinflusst (z. B. van den Bulck \& Beullens 2007). Diese Studien geben jedoch kaum Hinweise darauf, welche Informationen die Sendungen über die thematisierten Berufe zur Verfügung stellen. So werden Berufe im Fernsehen selten als tatsächliche Berufe thematisiert, man findet sie meistens nur als Begleiterscheinungen bzw. Folgen der Protagonisten oder Antagonisten, Themen und Handlungen, sodass Berufsbilder im Fernsehen überwiegend indirekt vermittelt werden (Krüger 2004). Dennoch ist nachgewiesen, dass die Fernsehpräsentation in der Lage ist, das Image eines Berufes gezielt zu beeinflussen (Michel \& Pelka 2004). Ziel des vorliegenden Beitrages soll es daher sein, zu klären, wie sich die Informationen über Berufe in Fernsehsendungen in den letzten 20 Jahren entwickelt haben. Dabei sind die drei folgenden Fragestellungen leitend für die Analyse:

1. Welches Spektrum an Berufen wird in populären Vorabendserien dargestellt?

2. Welches Spektrum an Tätigkeiten der einzelnen Berufe stellen populäre Vorabendserien dar?

3. Wie werden die Berufsumwelten der dargestellten Berufe charakterisiert?

Zur Beantwortung dieser Forschungsfragen geht der Beitrag zunächst auf den Berufswahlprozess ein und sichtet dann den Forschungsstand zur Berufsorientierung mit Medien mit besonderem Fokus auf Fernsehserien. Der Beitrag erläutert dann das Forschungsdesign der Studie, stellt ausgewählte Ergebnisse dar und diskutiert diese vor dem Hintergrund bestehender Erkenntnisse.

\section{Theoretischer und empirischer Hintergrund}

\subsection{Grundlagen der Berufsorientierung}

Der heutige Berufsbegriff setzt bei der Erwerbsarbeit an und gibt nach Kurtz (2002) die jeweilige Form der Arbeit an. Der „Beruf ist eine aus gesellschaftlichen Bildungsprozessen hervorgehende soziale Form spezifisch zugeschnittener Fähigkeiten und Fertigkeiten und dazu komplementärer fachlicher Tätig- 
keiten und Leistungen. Berufe werden zur Erfüllung gesellschaftlicher Funktionen und darüber [hinaus] zum Erwerb von Geldeinkommen von Personen übernommen. Die Berufstätigen werden dadurch gesellschaftlich eingebunden, sozialen Normen unterworfen und in wichtigen persönlichen Aspekten geprägt - sie wirken über die Ausübung ihres Berufs aber auch auf die Gesellschaft zurück.“ (Demszky \& Voß 2018, 477).

Für die Klärung, welchen Beruf1 ein Individuum ergreifen und realisieren möchte, existieren verschiedene theoretische Ansätze. Eine der einflussreichsten Berufswahltheorien stammt von Holland (1997). Diese Theorie basiert u. a. auf der Annahme, dass Menschen nach Berufen suchen, ,die es ihnen ermöglichen, ihre Fähigkeiten und Fertigkeiten anzuwenden, ihre Werthaltungen und Einstellungen auszudrücken sowie ihnen gemäße Probleme und Rollen zu übernehmen bzw. nicht-korrespondierende zu vermeiden" (Seifert 1977, 211). Das Modell unterscheidet sechs Persönlichkeitstypen bzw. berufliche Umwelten, die zur Klärung dieser Passung herangezogen werden.

Damit Jugendliche diese Passung herstellen können, benötigen sie unter anderem Kenntnisse über ihre eigenen Fähigkeiten, Werte und Ideale auf der einen Seite und Informationen über die berufsrelevanten Umwelten auf der anderen Seite (Blickle 2014). Eine wichtige Informationsquelle stellen hierzu Medien dar. So nehmen Medien bereits den vierten Platz zur Begründung von Berufswünschen nach den Eltern und eigenen Interessen, aber deutlich vor institutionalisierten Angeboten/Schule ein (Weyer, Gehrau \& Brüggemann 2016). Medien sind dabei besonders bedeutsam, wenn das Medium mit den Interessen eines Jugendlichen übereinstimmt und wenn Erfahrungen aus Praktika fehlen, d. h. die Informationen aus den Medien kompensieren fehlende praktische Erfahrungen (Weyer, Gehrau \& Brüggemann 2016).

\subsection{Empirischer Forschungsstand zur Berufsorientierung mit Medien}

Obgleich die vorrangige Bedeutung der Eltern, Familie und Freunde für die Genese der Berufsvorstellungen und die Realisation der Berufswahl junger Menschen unstrittig ist, kommt in der modernen Gesellschaft den (Massen-)Medien ein großer Einfluss auf die Meinungsbildungs- und damit auch auf die Berufswahlprozesse der jungen Menschen zu (Dostal 2006; Esch 2011; Hipeli \& Süss 2013).

Der Begriff des Mediums geht in seinem ursprünglichen Verständnis auf die Arbeiten der beiden US-amerikanischen Mathematiker Shannon

1 Somit ist der Beruf vom Job, als einer schnell zu erlernenden, kurzfristig zu erledigenden und wechselnde Tätigkeit zum Geld verdienen, abzugrenzen, bei der es nicht zu einer stabilen Identifikation mit der jeweiligen Aufgabe kommt (Dostal, Stooß \& Troll 1998). 
(1949/1998) und Weaver (1949/1998) zurück, wonach ein Medium als ein zwischen „transmitter [Kursiv im Original; Anm. d. Verf.] " und „receiver [Kursiv im Original; Anm. d. Verf.]“ gelegener „,communication channel [Kursiv im Original; Anm. d. Verf.]" verstanden wird. Einen wichtigen Einfluss für die Erarbeitung eines modernen Verständnisses des Begriffs des Mediums bildeten hier die Arbeiten des kanadischen Geisteswissenschaftlers McLuhan (1964/1975; s. a. McLuhan \& Fiore 1967/1971), wonach das eingesetzte Medium die transportierte Information verändert. Nach diesem Medienverständnis fügt das ausgewählte Medium der beförderten Nachricht immer auch eine dem Medium innewohnende Nachricht bei, wodurch die Medienauswahl einen großen Einfluss auf die erfolgreiche Realisation der verfolgten Absicht besitzt, unabhängig davon, ob diese ökonomischer, pädagogischer oder wie auch immer gelagerter Natur ist (McLuhan \& Fiore 1971; McLuhan 1975; Sesink 2007).

Insbesondere das Fernsehen besitzt für junge Menschen eine große Bedeutung und beeinflusst deren Vorstellungen und Erwartungen von der Gesellschaft und Arbeitswelt (Krüger 2004; Dostal 2006; Hipeli \& Süss 2013). Nach Bandura (2001a; 2001b) findet das soziale Lernen junger Menschen anhand realer oder fiktiver, bspw. medial vermittelter Modelle, also durch die Orientierung an und das Nachahmen von (positiven) Rollenvorbildern statt, denn dieser geht davon aus, dass junge Menschen nicht nur durch das Erleben eigener, sondern auch durch das Teilen, Reflektieren und Nachmachen fremder Erfahrungen lernen können. Sofern (positive) Rollenvorbilder in der Lebenswirklichkeit fehlen, können diese durch entsprechende der Fernsehwirklichkeit ersetzt werden (Esch \& Grosche 2011; Hipeli \& Süss 2013; Gehrau 2014).

Befragungen von jungen Menschen vor und nach ihrer Berufswahl haben aufgezeigt, dass der regelmäßige Konsum von Fernsehinhalten im Allgemeinen und von Fernsehserien, wie Daily Soaps bzw. Seifenopern, im Speziellen deren Berufswahl stark beeinflusst. So gaben im Rahmen der Schüler-Befragung des MINTiff-Projektes 23\% der jungen Frauen und 18\% der jungen Männer an, durch fiktionale Serien und Filme auf ihren späteren Berufswunsch aufmerksam geworden zu sein - mehr als bspw. durch Schulunterricht (Esch \& Grosche 2011).

Weiterhin kamen diverse Studien zu dem Ergebnis, dass in Daily Soaps soziale Berufe des tertiären Wirtschaftssektors (Dienstleistung), insbesondere der Bereiche Ordnung und Sicherheit, Geisteswissenschaften und Kunst, Medien und Unterhaltung, im Gegensatz zu produktiven Berufen des sekundären Sektors (Handwerk und Industrie) und des primären Sektors (Landwirtschaft) deutlich überrepräsentiert dargestellt werden (Krüger 2004; Michel \& Pelka 2004; Dostal 2006; Gehrau 2014). Dessen Ursache wird insbesondere in dem Umstand begründet gesehen, dass beim Fernsehen überwiegend Menschen arbeiten, die den Geisteswissenschaften oder der Kunst zuzurechnen sind (insb. 
Autor*innen, Produzent*innen, Regisseur*inne(n), Schauspieler*innen, welche ihre eigenen Berufe sehr gut kennen und ein realistisches Bild von ihnen zeichnen können, während sie von anderen Berufen in der Regel nur die in der Gesellschaft vorhandenen stereotypischen Vorstellungen teilen (Krüger 2004; Osterath 2010). So sind beispielsweise die dargestellten Berufe (in Seifenopern) zu fast 90\% Dienstleistungsberufe. In der Realität hingegen kommen Dienstleistungsberufe (1t. Stat. Bundesamt) lediglich auf 65,5\%. Die Serie „Gute Zeiten, Schlechte Zeiten“ thematisiert sehr häufig journalistische und künstlerische Berufe (Michel \& Pelka 2004). „Marienhof“ rückt hingegen Gesundheits-, Sozial- und Erziehungsberufe sowie geisteswissenschaftliche Berufe in den Vordergrund (Michel \& Pelka 2004). Es kommt somit zu einer Überschätzung des tatsächlichen Anteils dieser Berufe in der Gesellschaft.

Ferner besitzen die Ausrichtungen, die Zielgruppen und die dementsprechenden Programmstrukturen der Sender, also die verwendeten TV-Formate und dargestellten Genres, einen großen Einfluss auf die Darstellungen der Berufsstruktur (so zeigen Privatsender bevorzugt Angebote der Genres Science Fiction und Sitcom, die öffentlich-rechtlichen Sender bevorzugen Angebote des Genres Alltag/Familie) (Esch 2011; Krüger 2004). Dies führt dazu, dass auch in den Köpfen der jungen Zuschauer*innen ein realitätsfernes Bild von der Verteilung und den Merkmalen der Berufe in der Arbeitswelt entsteht (Dostal \& Troll 2004; Dostal 2006).

Dabei ist jedoch zu beachten: Je höher der Fernsehkonsum ist, desto höher ist der Fernseheinfluss (Dostal 2006) und es ist anzumerken, dass die Fernsehrealität nicht immer der Lebensrealität in vollem Umfang entspricht (Dostal \& Troll 2004; Dostal 2006; Esch 2011; Krüger 2004). Für die Wahl des Berufs durch die jungen Menschen kommt es in einem ersten Schritt allerdings nicht darauf an, dass dieser realistisch dargestellt wird, sondern, dass er attraktiv erscheint (Michel \& Pelka 2004; Osterath 2010). Und auch die Fremdaufsicht durch den Staat sowie die Selbstkontrolle durch die TV-Anstalten haben sicherzustellen, dass wichtige soziale Themenbereiche realitätsnah und verantwortungsvoll dargestellt werden.

Schließlich konnten Studien nachweisen, dass der Konsum von Sendungen, die einen bestimmten Beruf thematisieren, tendenziell eine verstärkende Wirkung ausüben, d. h. die Chance erhöhen, dass Jugendliche genau den dargestellten Beruf erlernen möchten (Gehrau \& Hofe 2013; van den Bulck \& Beullens 2007).

Aus diesem Forschungsstand ergeben sich grundlegende Annahmen bei der Analyse der drei Forschungsfragen:

1. Darstellungen von Berufen in Fernsehserien können Informationen über Berufe bereitstellen, die für Jugendliche ausschlaggebend für oder gegen die Wahl eines bestimmten Berufs sein können (dazu z. B. Gehrau \& Hofe 2013; van den Bulck \& Beullens 2007). 
2. Berufsdarstellungen aus Fernsehserien können bei der Berufswahl fehlende praktische Erfahrungen zu einem Beruf kompensieren (Weyer, Gehrau \& Brüggemann 2016)

3. Jugendliche suchen vor allem Medien auf und nutzen diese zur Berufswahlbegründung, wenn sie zu ihren Interessen passen (Weyer, Gehrau \& Brüggemann 2016).

Das aus diesen Annahmen entstandene Forschungsdesign zur Beantwortung der Fragen wird im nachfolgenden Abschnitt dargestellt.

\section{Methodisches Vorgehen}

Zur Beantwortung der Forschungsfragen wurden insgesamt 40 Folgen von Gute Zeiten, Schlechte Zeiten (GZSZ) und 40 Folgen von Unter Uns mittels eines Kodierleitfadens inhaltsanalytisch ausgewertet. Die Auswahl der Serien GZSZ und Unter $U n s^{2}$ erfolgte, da es aktuell die am längsten ununterbrochen ausgestrahlten Serien dieser Art in Deutschland sind: GZSZ seit 1992, Unter uns seit 1994. ${ }^{3}$ Insgesamt entsteht über die hier getroffene Auswahl somit die Möglichkeit der Umfassung eines möglichst großen Zeitraums. Darüber hinaus bieten die Serien den Vorteil einer breiten Abdeckung an Themen und Regionen, da GZSZ in Berlin angesiedelt ist und ein jugendliches Klientel darstellt, das an der Schwelle des Übergangs ins Berufsleben steht. Konkret dargestellt wird das alltägliche Leben mit seinen Höhen und Tiefen von Schüler*innen und jungen Berufstätigen im sozialen Jahr oder der Medienbranche, dem Modebereich, der Gastronomie sowie dem Handwerk. Unter Uns spielt dagegen in einer Kölner Bäcker- und Konditorfamilie und stellt den beruflichen und privaten Alltag einer Familie mit insgesamt drei Generationen dar.

Aus der qualitativen Inhaltsanalyse ergaben sich in Summe 1.328 Kodierungen (764 Kodierungen bei GZSZ und 564 Kodierungen bei Unter Uns). Für die Auswahl der Folgen wurde der Ausstrahlungszeitraum der beiden Serien in 4 gleichgroße Quartale eingeteilt. Aus dem ersten Quartal jeder Serie wurde die erste Staffel für die Analyse ausgewählt - für GZSZ ist dies das Jahr 1992 und für Unter Uns das Jahr 1994/1995.

2 Die Relevanz beider Serien wird auch durch die zweistelligen Einschaltquoten bestätigt GZSZ: 2018, 2,84 Mio., Unter Uns: 2018: 1,07 Mio. ${ }^{2}$, wobei die jugendliche Zuschauerschaft tendenziell eher weiblich geprägt ist (BR-Online 2002).

3 Andere über lange Jahre erfolgreiche Serien wie Marienhof oder Verbotenen Liebe mit jeweils mehr als 4.000 Folgen sind hingegen bereits seit längerem eingestellt worden, sodass hier bei einer Analyse keine Aktualität gegeben wäre. Weitere noch laufende Serien wie Berlin - Tag \& Nacht oder Köln 50667 bieten wiederum nur deutlich kürzere Zeitreihen. 
Aus den beiden mittleren Quartalen wurden jeweils Folgen ausgewählt, die möglichst mittig in dem jeweiligen Zeitraum ausgestrahlt wurden. Für das letzte Quartal wurden die aktuellsten Folgen zum Zeitpunkt der Kodierung für die Analyse genutzt. Jede Folge wurde mit einem standardisierten inhaltsanalytischen Schema ausgewertet, welches nach den Kriterien für die skalierende Strukturierung nach Mayring (2015) entwickelt wurde. Jede Darstellung einer beruflichen Tätigkeit löste eine Kodierung aus, bei der die Zeitstelle in der Serie, eine potentielle Berufs- bzw. Jobbezeichnung sowie die gezeigten Tätigkeiten, Arbeitsbedingungen, Arbeitsmittel und Arbeitsumgebungen in dem Schema festgehalten wurden. Darüber hinaus wurde kodiert, ob die Tätigkeit von einem Mann oder einer Frau durchgeführt wurde, wie alt die darstellende Person ungefähr war und welches Persönlichkeitsmerkmal nach dem Modell von Holland (1985) die jeweilige Szene suggeriert $(0=$ Merkmal nicht erkennbar, 1 = Merkmal erkennbar). Auf der Grundlage dieser Daten wurden die einzelnen Kodierungen in die Systematik der Berufe und Anforderungen (KldB 2010) der Bundesagentur für Arbeit eingeordnet.

In einem weiteren Schritt wurden die 1.328 Kodierungen zu Berufsprofilen weiter verdichtet. Hierzu erfolgte für jeden Beruf ein Abgleich der dargestellten Tätigkeiten mit den Tätigkeiten, die laut berufe.net die Kerntätigkeit des jeweiligen Berufes ausmachen. Auf diese Weise lässt sich abschätzen, in welchem Umfang die Berufsdarstellungen in den Serien das Tätigkeitsspektrum der Berufe abdecken. Die Ergebnisse werden im Folgenden dargestellt.

\section{Darstellung der Ergebnisse}

\subsection{Ergebnisse zum dargestellten Berufsspektrum}

Welche Berufshauptgruppen in den beiden Serien thematisiert werden, zeigt die nachfolgende Abbildung 2. 
Abb. 2 Entwicklung der Berufsbereiche in der Serie „,GZSZ“

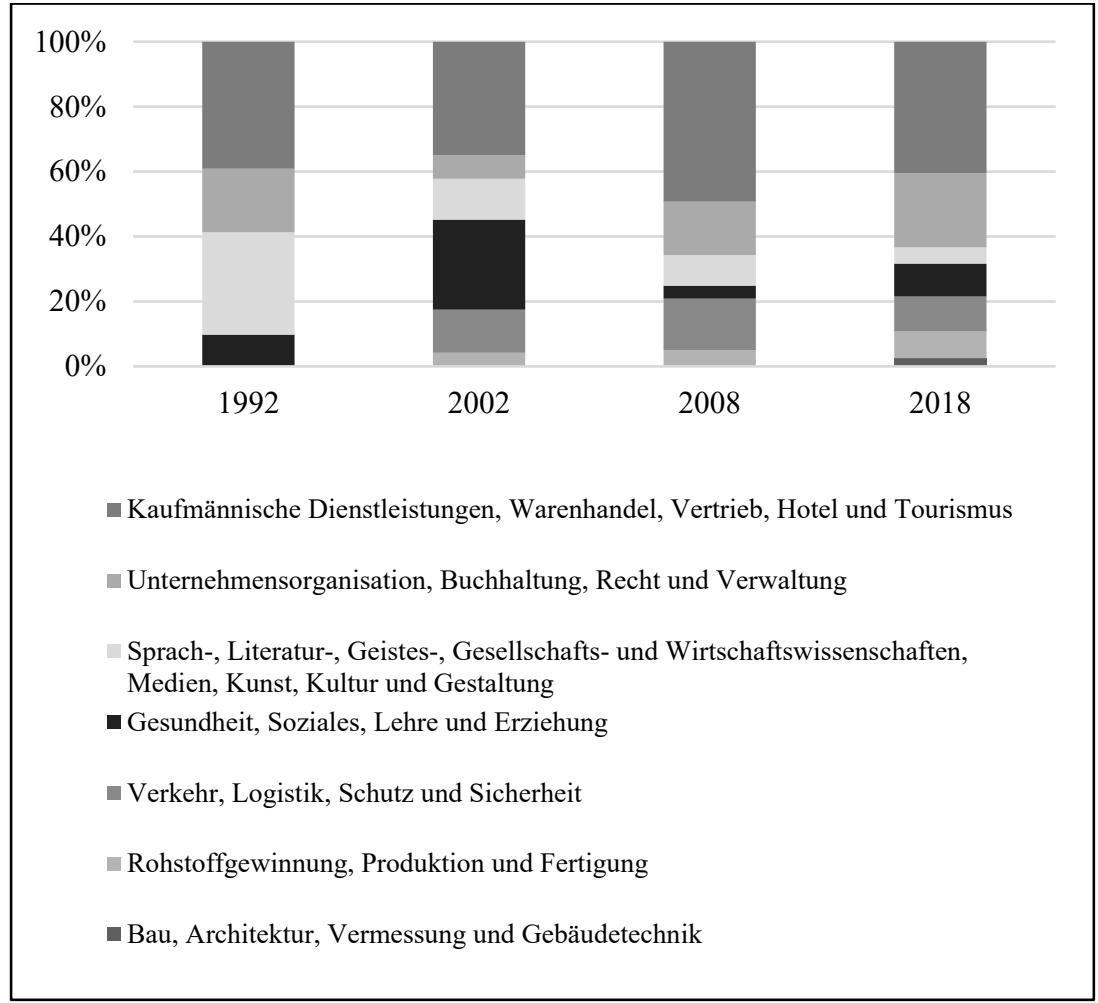

Quelle: eigene Darstellung

Es zeigt sich, dass die kaufmännischen Dienstleistungsberufe die ersten drei Quartale von GZSZ dominieren und ihr Anteil sogar noch wächst, während im vierten Quartal eine Verschiebung zugunsten von Berufen in der „Unternehmensorganisation, Buchhaltung, Recht und Verwaltung" erfolgt. Berufe aus den Sektoren „Verkehr, Logistik, Schutz und Sicherheit“ bleiben hingegen konstant wie auch der Anteil der Szenen aus dem Bereich „Rohstoffgewinnung, Produktion und Fertigung“. Berufe mit Bezug zu „Bau, Architektur, Vermessung und Gebäudetechnik" kommen in der Serie GZSZ kaum vor. Mit der Zeit haben hingegen Darstellungen in den Berufsgruppen „Sprach-, Literatur-, Geistes-, Gesellschafts- und Wirtschaftswissenschaften, Medien, Kunst, Kultur und Gestaltung“" sowie „Gesundheit, Soziales, Lehre und Erziehung“ 
abgenommen. Berufe aus dem Bereich „Gesundheit, Soziales, Lehre und Erziehung" haben in der Regel einen geringen Anteil mit Ausnahme des zweiten Quartals, in dem es einen Ausreißer des Anteils nach oben gibt.

Auch bei Unter Uns liegt der Schwerpunkt auf den kaufmännischen Dienstleistungsberufen, die bis Quartal 3 zugenommen haben. Und ebenfalls haben im vierten Quartal die kaufmännischen Dienstleistungsberufe im Vergleich zu Berufen der „Unternehmensorganisation, Buchhaltung, Recht und Verwaltung" an Anteilen verloren. ,Verkehr, Logistik, Schutz und Sicherheit“" hingegen spielen eine geringere Rolle als bei GZSZ. Im Gegensatz dazu tauchen Berufe der „Rohstoffgewinnung, Produktion und Fertigung“ deutlich häufiger auf, nehmen aber gleichzeitig im Zeitverlauf wieder ab. Einen konstant geringen Anteil weisen die Berufe der „Gesundheit, Soziales, Lehre und Erziehung“ und auch diejenigen für „Verkehr, Logistik, Schutz und Sicherheit" auf. In welchem Ausmaß die Tätigkeiten der Berufe dargestellt werden, verdeutlicht der nachfolgende Abschnitt.

\subsection{Ergebnisse zum dargestellten Tätigkeitsspektrum der Berufe}

Die zweite Forschungsfrage ermittelt, welches Spektrum an Tätigkeiten der einzelnen Berufe populäre Vorabendserien darstellen. Abbildung 6 zeigt zur Beantwortung zunächst getrennt für die beiden Serien, wie sich der Umfang der dargestellten Berufstätigkeiten in den analysierten Serien entwickelt hat.

Die Analysen zeigen, dass das Ausmaß an Übereinstimmung im Durchschnitt sehr gering ist. So reicht die durchschnittliche Übereinstimmung für GZSZ von ca. $4 \%$ bis maximal 15\%. Bei Unter Uns reicht die Spanne von 5,7\% bis ca. 11\%. Lediglich bei Unter Uns lässt sich der Trend erkennen, dass die durchschnittliche Übereinstimmung mit der Zeit abnimmt. Bei der Interpretation der Ergebnisse ist aber zu berücksichtigen, dass für alle Serien eine hohe Standardabweichung festzuhalten ist, die teilweise bis zu 29\%-Punkte umfasst. Dies bedeutet, dass es sehr wohl vereinzelt Berufsdarstellungen in den Serien gibt, die eine relativ hohe Übereinstimmung mit den Berufsbeschreibungen der Bundesagentur für Arbeit aufweisen. Einen konkreten Einblick in das unterschiedliche Ausmaß der Abbildung von Tätigkeiten in den Serien verdeutlich exemplarisch die nachfolgende Abbildung 3. 
Abb. 3: Ausmaß der Übereinstimmung der Tätigkeiten für den Beruf , Bäcker*in “ in der Serie ,Unter Uns“

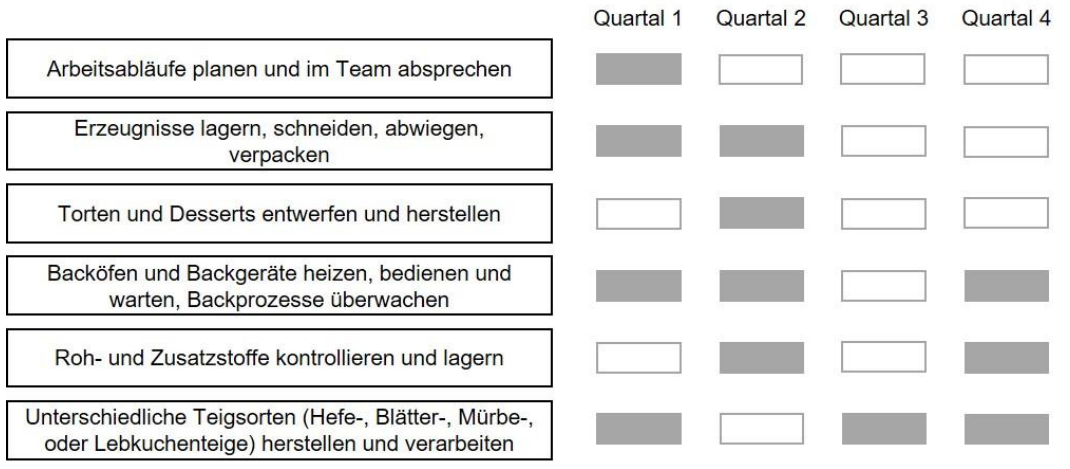

Quelle: eigene Darstellung

So zeigt Abbildung 3, dass maximal 4 von 6 Tätigkeiten von Bäcker*innen in den Folgen zu erkennen sind. Die erkennbaren Tätigkeiten schwanken im Zeitablauf deutlich. Konstanten gibt es bei diesem Beruf im Wesentlichen in der Herstellung verschiedener Teigsorten und der Bedingung der notwendigen Geräte zum Backen. Ein ähnliches Bild zeigt auch Abbildung 8 im Vergleich der Serien zusammengefasst über die Zeit. Wie sich die Charakterisierung der Berufsumwelt in den Serien unterscheidet und entwickelt, analysiert der nachfolgende Abschnitt.

\subsection{Ergebnisse zur Charakterisierung der Berufsumwelten}

Wie die Serien die Berufsumwelten darstellen (Forschungsfrage 3), wird mit Tabelle 1 über eine mehrfaktorielle Varianzanalyse untersucht. Dabei sind Einflüsse auf dem 1\%- oder 5\%-Niveau grau hinterlegt, um eine bessere Orientierung zu ermöglichen. Nach Cohen (1988, 283 ff.) zeigt ein $\eta^{2}$ von mindestens .001 einen schwachen, von .06 einen mittleren und von .14 einen starken Effekt an. Aus Platzgründen werden nur die Effektstärken dargestellt. 
Tab. 1: Einfluss verschiedener Faktoren auf die Berufsmerkmale - Effektgröße $\eta^{2}$

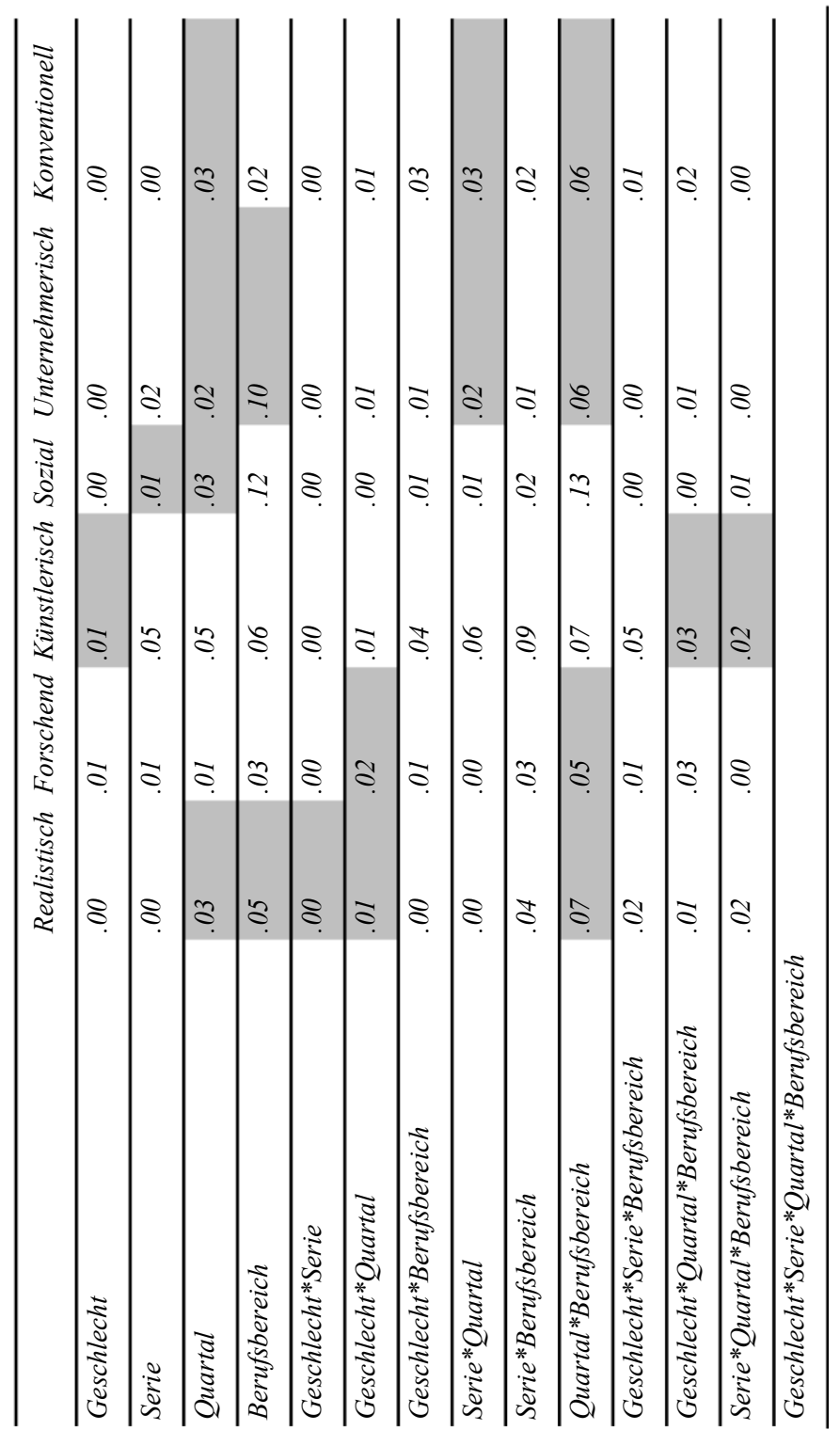

Quelle: eigene Darstellung 
Tabelle 1 zeigt zunächst, dass es keinen Einflussfaktor gibt, der für alle Berufsumwelten gleichermaßen bedeutsam ist. Werden die Effektgrößen aus der Tabelle 1 zur weiteren Eingrenzung von praktisch bedeutsamen Einflüssen hinzugezogen, so zeigt sich, dass der Berufsbereich für das Merkmal „realistisch" und „unternehmerisch“"zwischen 5\% und 10\% der Variation erklärt und damit ein mittlerer bis starker Effekt vorliegt. Weiterhin kann die Interaktion aus zeitlichem Verlauf und Berufsbereich bei allen Merkmalen mit Ausnahme der Merkmale „künstlerisch“ und „sozial“ zwischen 5\% und 7\% der Variation erklären.

Das Geschlecht der Schauspieler*innen der Tätigkeiten wird zudem oft als Interaktionseffekt und einmal als Haupteffekt für das Merkmal „künstlerisch“ signifikant. Werden jedoch die Effektgrößen zur weiteren Interpretation herangezogen zeigt sich, dass der Haupteffekt nur 1\% der Unterschiede im Merkmal „,künstlerisch“ erklären kann. Für das Merkmal „realistisch“ ist die Effektgröße über alle signifikanten Einflüsse, die das Geschlecht beinhalten bei 1\% und für ,forschend“ bei $2 \%$. Insgesamt sind damit keine praktisch bedeutsamen Unterschiede in den Charakteristika zwischen den Darstellungen von Männern und Frauen in den Serien feststellbar. Vor diesem Hintergrund konzentriert sich die weitere Analyse auf die exemplarische Darstellung eines Einflusses mit großer Varianzaufklärung (vgl. Abb. 4).

Abb. 4: Variation der Merkmale Realistisch und Unternehmerisch in Abhängigkeit vom Berufsbereich

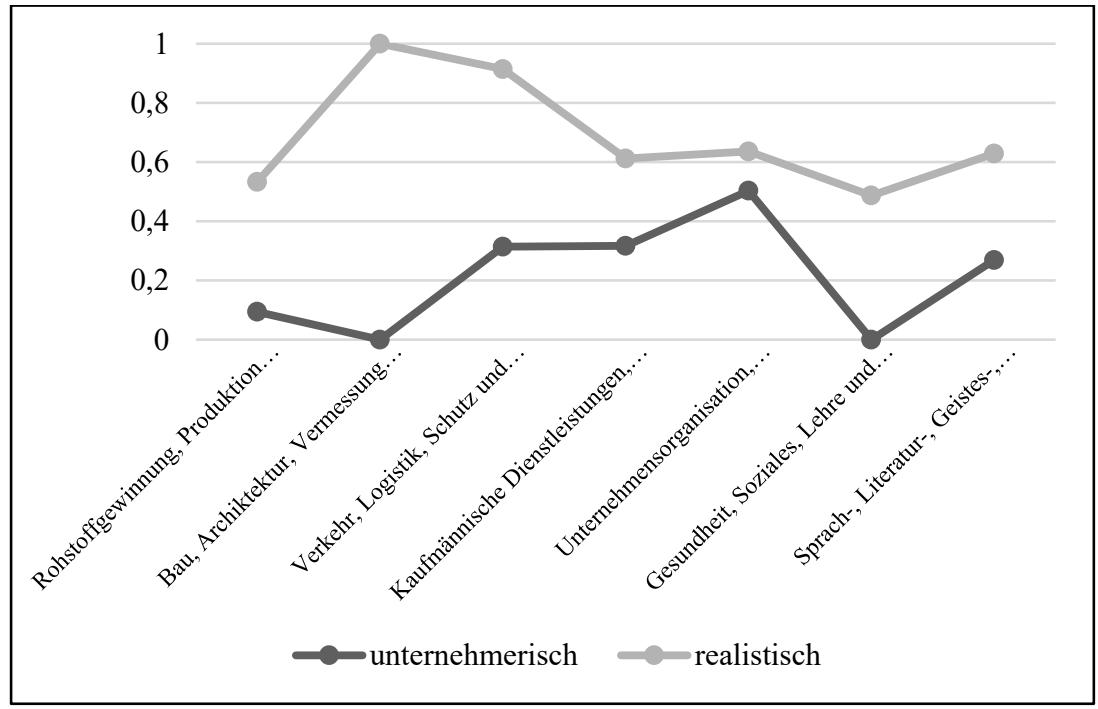

Quelle: Eigene Darstellung 
Die Abbildung 4 zeigt den Einfluss des Berufsbereiches auf die Charakterisierung der Berufsdarstellung als „unternehmerisch“ und als ,realistisch“. Es wird deutlich, dass vor allem die Berufsbereiche „Bau, Architektur, Vermessung und Gebäudetechnik" sowie „Verkehr, Logistik, Schutz und Sicherheit" als ,realistisch“ dargestellt werden. Im Gegensatz dazu werden die Berufe des Bereiches „Gesundheit, Soziales, Lehre und Erziehung“ als am wenigsten realistisch eingeschätzt. Im Bereich der Darstellung als „unternehmerisch“ erhalten vor allem Berufe des Bereiches „Unternehmensorganisation, Buchhaltung, Recht und Verwaltung“" sowie die weiteren kaufmännischen Berufe eine solche Einschätzung. Am wenigsten ,unternehmerisch“ werden hingegen der Berufsbereich „Bau, Architektur“ sowie „Gesundheit, Soziales, Lehre und Erziehung" angesehen.

\section{Diskussion}

Studien der Vergangenheit konnten zeigen, dass die Betrachtung von Fernsehsendungen einen Einfluss auf Berufswahlentscheidungen Jugendlicher nimmt und die Stärke des Einflusses tendenziell mit einem gesteigerten Konsum zunimmt (Gehrau \& vom Hofe 2013; van den Bulck \& Beullens 2007). Auch zeigt die Studie vom Weyer, Gehrau und Brüggemann (2016), dass Medien bei der Begründung von Berufswünschen eine mittlere bis hohe Bedeutung annehmen. Dies gilt insbesondere dann, wenn Erfahrungen aus Praktika zur Berufswelt fehlen. Dies deutet darauf hin, dass Jugendliche Informationen über Berufe aus Medien sammeln und für ihre Berufswahlentscheidungen nutzen. Bisherige Studien zum Einfluss von Fernsehserien auf die Berufswahl haben die Informationen, die in Sendungen dargestellt werden, nur randständig analysiert. Diese Lücke greift der vorliegende Beitrag auf.

Fragestellung 1: Deutlich wird bei der Datenanalyse zunächst, dass eine große Anzahl an Berufen dargestellt wird. Bei GZSZ werden insgesamt 47 verschiedene Berufe erkennbar, bei Unter Uns hingegen nur 42. Der Schwerpunkt bei GZSZ und Unter Uns liegt aber deutlich auf kaufmännisch-verwaltenden Berufen. Damit zeigen sie nur einen kleinen Teil möglicher Berufe. Dies passt zu den Studienergebnissen von Michel und Pelka (2004), die ebenfalls feststellen, dass in Daily Soaps v. a. Dienstleistungsberufe thematisiert werden.

Fragestellung 2: Obwohl die Serien sich somit auf ein eher enges Spektrum an Berufen konzentrieren, zeigt die tiefergehende Analyse der Tätigkeiten, dass diese ebenfalls nur Ausschnitte aus den Berufen präsentieren. So liegt der mittlere Anteil an dargestellten Tätigkeiten bei nicht mehr als 15\%. Im Durchschnitt werden also nur rund maximal 15\% der Tätigkeiten aus den Berufen erkennbar. Dies gilt für den gesamten analysierten Zeitraum. So werden beispielsweise nur Ausschnitte der Tätigkeiten von Bäcker*innen in der Serie 
Unter Uns sichtbar (vgl. Abb. 3), obwohl diese Serie gerade eine Bäckerfamilie thematisiert. Damit liegt im Ergebnis eine doppelte Einschränkung vor, einerseits bei dem Spektrum der dargestellten Berufe und andererseits bei den dargestellten Tätigkeiten der Berufe.

Fragestellung 3: Etwas anders erscheint die Situation bei der Charakterisierung der beruflichen Umwelten auf der Basis des Modells von Holland (1963). So zeigt die Analyse hier, dass v. a. die Berufsbereiche „Bau, Architektur, Vermessung und Gebäudetechnik“ sowie „Verkehr, Logistik, Schutz und Sicherheit" als realistisch beschrieben werden. Dies passt durchaus zu dem Modell von Holland (1963), da zu diesem Merkmal Berufe wie LKW-Fahrer*innen, Tischler*innen, Geräteführer*innen oder auch Landwirte zählen. Im Gegensatz dazu werden die Berufe des Bereiches „Gesundheit, Soziales, Lehre und Erziehung" als am wenigsten realistisch eingeschätzt. Dies ist nicht verwunderlich, da sich Lehrer*innen, Sozialarbeiter*innen, Krankenpfleger*innen und Therapeut*innen häufig dem sozialen Persönlichkeitstyp zuordnen lassen (Holland 1963).

Im Bereich der Darstellung als ,unternehmerisch“erhalten v. a. Berufe des Bereiches „Unternehmensorganisation, Buchhaltung, Recht und Verwaltung“ sowie weitere kaufmännischen Berufe eine solche Einschätzung. Berufliche Umwelten dieses Typus beschäftigen sich mit Macht-, Status- und Führungsproblemen und Persönlichkeiten dieses Typus schreiben sich selbst dominante und führende Fähigkeiten zu. Sie haben häufig den Wunsch, Politiker*in, Unternehmer*in oder Industrieberater*in zu werden (Holland 1963; Scheller 1976; Beinke 1999). Somit korrespondieren auch diese Charakteristika mit Berufen dieses Berufsbereiches, dennoch wäre zu erwarten gewesen, dass ebenfalls eine hohe Einschätzung im Bereich des angepassten Persönlichkeitstypus liegt, da hier Buchhalter*innen, Statistiker*innen, Steuerberater*innen oder Banker*innen zugeordnet werden können (Holland 1963; BA 2011). Am wenigsten ,unternehmerisch“ werden hingegen der Berufsbereich „Bau, Architektur" usw. sowie „Gesundheit, Soziales, Lehre und Erziehung" angesehen. Somit passt ein Teil der Charakteristika zu den Berufen.

Obwohl also die Tätigkeiten nicht umfassend dargestellt werden, gelingt es den Serien scheinbar, die Art der beruflichen Umwelt so zu charakterisieren, wie es näherungsweise dem Modell von Holland (1963) entspricht. Möglicherweise lassen sich diese grundlegenden Typen leichter in Fernsehsendungen implementieren als detaillierte Handlungen einzelner beruflicher Tätigkeiten, die ein entsprechendes Fachwissen zu diesen Tätigkeiten voraussetzen.

Überraschend ist in diesem Zusammenhang, dass das Geschlecht keinen praktisch bedeutsamen Anteil an der Variation in den Merkmalen erklärt. Dies bedeutet, dass sich die Merkmalsdarstellungen nicht systematisch zwischen Frauen und Männern unterscheiden. Den Serien ist es scheinbar gelungen, Stereotypen bei den beruflichen Tätigkeiten zu vermeiden. 
Damit untermauert diese Studie, dass die Informationen, die Fernsehsendungen Jugendlichen zur Verfügung stehen, in den letzten 20 Jahre keine belastbare Wissensbasis über die berufliche Welt zur Verfügung stellen. Für den berufsorientierten Unterricht ergibt sich daraus die Konsequenz, dass Jugendliche für die verzerrte bzw. unvollständige Darstellung von Berufen in diesem Medium zu sensibilisieren sind, wie sich z. B. auch die Studie von Gehrau und vom Hofe (2013) berichtet. Hierzu könnten im Unterricht entsprechende Serien betrachtet, analysiert und mit den Tätigkeitsbeschreibungen von Berufe.Net oder Praktika abgeglichen werden. Für die weitere Forschung ergibt sich hieraus der Bedarf zu klären, wie Berufe dargestellt werden, um daraus Konzepte für den berufsorientierenden Unterricht zu entwickeln. So zeigt beispielsweise die Studie von Jahncke et al. (2020), dass Jugendliche beruflich relevante Informationen auch über Instagram wahrnehmen und suchen und diese vor allem bei Jugendlichen mit einem Realschulabschluss zu einer Bestärkung eines Berufswunsches beitragen, unabhängig davon, wie erfolgsversprechend die Realisation des Berufswunsches ist.

Limitationen: Die Studie ist nicht ohne Limitationen. Eine wesentliche Grenze ergibt sich aus dem verwendeten Berufswahlmodell von Holland. Hierzu zählt, dass eine rein statische Betrachtung hinsichtlich der Persönlichkeitsstruktur und der Umweltstruktur vorgenommen wird (im Folgenden auch Porath 2013). Auch das der Interaktion zugrundeliegende Kausalverhältnis berücksichtigt keine Veränderungen der Umwelt oder der Persönlichkeitsmerkmale (Beinke 1999; Potocnik 1990; Ries 1970). Der Prozess der Entscheidungsfindung entspricht auch hier eher einer Blackbox, wodurch nicht geklärt wird, ob ein Individuum tatsächlich versucht, eine kongruente Interaktion herzustellen (Beinke 1999; Scheller 1976). Die Aussagen zu den Zufriedenheits-, Stabilitäts- und Erfolgsprognosen hat Holland selbst gemäß Bußhoff (2009) durch zahlreiche Untersuchungen teilweise widerlegt. So hat der Grad der Kongruenz zwar eine starke empirische Unterstützung hinsichtlich der Zufriedenheit, aber nur einen geringen Erklärungsgehalt für die Berufsstabilität und den Berufserfolg (Bußhoff 1984). Die Bedeutung von Aspekten wie die Dauer der Beschäftigung, die Anzahl der Berufsalternativen, die Tendenz zur Anpassung, um keine Inkongruenzen erleben zu müssen, die persönliche Disposition zur Zufriedenheit oder auch das Alter wird anerkannt, sodass Matching eher als Suchtendenz zu verstehen ist, bei der das Individuum eine Umwelt sucht, in der es günstige Bedingungen für eine wechselseitige Anpassung zwischen Person und beruflicher Umwelt bzw. Beruf vorfindet. Es entsteht somit eine Anpassungsdynamik, in der eine scheinbar kongruente berufliche Option erprobt wird. Bei Zufriedenheit wird diese beibehalten und bei Unzufriedenheit verändert oder ganz aufgegeben. Diese Anpassungsprozesse erfolgen so lange, bis das Individuum eine subjektiv zufriedenstellende Person-Umwelt-Balance hergestellt hat (Bußhoff 2009). 


\section{Literatur}

Bundesagentur für Arbeit (BA) (2011). Klassifikation der Berufe 2010. Band 1: Systematischer und alphabetischer Teil mit Erläuterungen. Nürnberg.

Bandura, A. (2001a). Social cognitive theory of mass communication. Mediapsychology, 3, 265-299.

Bandura, A. (2001b). Social cognitive theory: an agentic perspective. Annual Review of Psychology, 52, $1-26$.

Beinke, L. (1999). Berufswahl. Der Weg zur Berufstätigkeit. Bad Honnef: Bock.

Blickle, G. (2014). Berufswahl und berufliche Entwicklung. In F. W. Nerdinger, G. Blickle \& N. Schaper (Hrsg.), Arbeits- und Organisationspsychologie (3. Aufl., S. 185-206). Berlin: Springer.

BR-Online (2002) IZI-Forschung - Sonstige Forschungsprojekte. Online: https://www.bronline.de/jugend/izi/deutsch/forschung/Sonstige_Forschungsprojekte/daily_soaps.htm (09.04.2020).

Brüggemann, T. \& Rahn, A. (2013). Zur Einführung: Der Übergang Schule-Beruf als gesellschaftliche Herausforderung - Entwicklung, rechtliche Verankerung und pädagogischer Auftrag der Berufsorientierung. In T. Brüggemann \& S. Rahn (Hrsg.), Berufsorientierung (S. 11-20). Münster: Waxmann.

Bußhoff, L. (1984). Berufswahl. Theorien und ihre Bedeutung für die Praxis der Berufsberatung (2. Aufl.). Stuttgart: Kohlhammer.

Bußhoff, L. (2009). Berufsberatung als Unterstützung von Übergängen in der beruflichen Entwicklung. In R. Zihlmann (Hrsg.), Berufswahl in Theorie und Praxis. Konzepte zur Berufswahlvorbereitung und Beratung unter veränderten wirtschaftlichen und gesellschaftlichen Bedingungen (3. Aufl., S. 9-77). Bern: SDBB.

Cohen, J. (1988). Statistical power analysis for the behavioral sciences $\left(2^{\text {nd }}\right.$ ed.). New York: Psychology Press.

Demszky, A. \& Voß, G. G. (2018). Beruf und Profession. In F. Böhle, G. G. Voß \& G. Wachtler (Hrsg.), Handbuch Arbeitssoziologie. Band 2: Akteure und Institutionen (2. Aufl., S. 477-538). Wiesbaden: Springer.

Dostal, W., Stooß, D. \& Troll, L. (1998). Beruf - Auflösungstendenzen und erneute Konsolidierung. Mitteilungen aus der Arbeitsmarkt- und Berufsforschung, 31, 438-460.

Dostal, W. \& Troll, L. (2004). Die Berufswelt im Fernsehen. Folgen für das Berufsverständnis und den Berufswahlprozess? ibv, 24, 57-58.

Dostal, W. (2006). Der Einfluss des Fernsehens auf das Berufswahlverhalten. In N. Bley \& M. Rullmann (Hrsg.), Übergang Schule und Beruf. Aus der Praxis für die Praxis - Region Emscher-Lippe. Wissenswertes für Lehrkräfte und Eltern (S. 305314). Recklinghausen: Forschungsinstitut Arbeit, Bildung, Partizipation.

Esch, M. (2011). MINT und Chancengleichheit in fiktionalen Fernsehformaten - Einführung und ausgewählte Ergebnisse einer Programmanalyse. In Bundesministerium für Bildung und Forschung (BMBF) (Hrsg.), MINT und Chancengleichheit in fiktionalen Fernsehformaten (S. 6-15). Bonn \& Berlin: W. Bertelsmann. 
Esch, M. \& Grosche, J. (2011). Fiktionale Fernsehprogramme im Berufsfindungsprozess - Ausgewählte Ergebnisse einer bundesweiten Befragung von Jugendlichen. In Bundesministerium für Bildung und Forschung (BMBF) (Hrsg.), MINT und Chancengleichheit in fiktionalen Fernsehformaten (S. 16-31). Bonn \& Berlin: W. Bertelsmann.

Feierabend, S., Plankenhorn, T \& Rathgeb, T. (2017) KIM-Studie 2016. Kindheit, Internet, Medien. Online: https://www.mpfs.de/fileadmin/files/Studien/KIM/2016/ KIM_2016_Web-PDF.pdf(14.04.2020).

Feierabend, S., Rathgeb, T. \& Reutter, T. (2020). JIM-Studie 2019. Jugend, Information, Medien. Online: https://www.mpfs.de/fileadmin/files/Studien/JIM/2019/ JIM_2019.pdf(14.04.2020).

Gehrau, V. \& vom Hofe, H. J. (2013). Medien und Berufsvorstellung Jugendlicher. Eine Studie zur Darstellung von Berufen in Fernsehserien und deren Einfluss auf die Berufsvorstellungen Jugendlicher. In T. Brüggemann \& S. Rahn (Hrsg.), Berufsorientierung. Ein Lehr- und Arbeitsbuch (S. 123-133). Münster: Waxmann.

Gehrau, V. (2014). Kultivierungseffekte des Fernsehens auf die Berufsvorstellungen von jugendlichen. $M \& K, 62(3), 417-438$.

Hartkopf, E. (2013). Berufswahlreife und Berufswahlkompetenz - zwei Schlüsselbegriffe der Berufswahlforschung und der Berufsorientierungspraxis aus psychologischer und pädagogischer Perspektive. In T. Brüggemann \& S. Rahn (Hrsg.), Berufsorientierung. Ein Lehr- und Arbeitsbuch (S. 42-57). Münster: Waxmann.

Hipeli, E. \& Süss, D. (2013). Werther, Soap Stars und Ego-Shooter-Helden: Das Einflusspotenzial medialer Vorbilder. In W. Schweiger \& A. Fahr (Hrsg.), Handbuch Medienwirkungsforschung (S. 191-205). Wiesbaden: Springer Fachmedien.

Holland, J. L. (1963). Explorations of a theory of vocational choice and achievement: II. A four-year predicition study. Psychological Reports, 12, 547-594.

Holland, J. L. (1985). Making vocational choices: A theory of vocational personalities and work environments. Englewood Cliffs, NJ: Prentice-Hall.

Holland, J. L. (1997). Making vocational choices. A theory of vocational personalities and work environments ( $3^{\text {rd }}$ ed.). Odessa: Psychological Assessment Resources.

Jahncke, H., Berding, F., Albers, K., Logemann, S. \& Rebmann, K. (2020). Die Rolle der Social-Media-Anwendung Instagram bei der Berufswahlentscheidung von Jugendlichen. Zeitschrift für Berufs- und Wirtschaftspädagogik, 116(1), 57-90.

Krüger, U. M. (2004). Berufe im Fernsehen: Ergebnisse einer Pilotstudie. Kurzfassung. $i b v, 24,58-65$.

Kurtz, T. (2002). Berufssoziologie. Bielefeld: transcript.

Mayring, P. (2015). Qualitaitve Inhaltsnalyse (12. Aufl.). Weinheim: Beltz.

McLuhan, M. \& Fiore, Q. (1971). The Medium is the Massage. An Inventory of Effects. Harmonsworth: Penguin Books.

McLuhan, M. (1975). Understanding Media. The Extensions of Man (5 $5^{\text {th }}$ ed.). London: Routledge \& Kegan Paul.

Michel, L. P. \& Pelka, B. (2004). Die Darstellung von Berufen im Fernsehen und ihre Auswirkungen auf die Berufswahl: Ergebnisse einer Pilotstudie. Kurzfassung. $i b v$, 24, 65-69.

mpfs (Medienpädagogischer Forschungsverbund Südwest) (2018). JIM 2018. Jugend, Information, Medien. Basisuntersuchung zum Medienumgang 12- bis 19-Jähriger in Deutschland. Stuttgart. 
Osterath, B. (2010). Chemikerinnen? Fehlanzeige! Nachrichten aus der Chemie, 58, 1234-1236.

Porath, J. (2013). Beförderung von Arbeits- und Berufsorientierung bei Schüler(inne)n der Berufsfachschule durch den Einsatz von Lernaufgaben. Eine konstruktiv-evaluative Studie. München: Hampp.

Potocnik, R. (1990). Entscheidungstraining zur Berufs- und Studienwahl. Theorie Konzeption - Evaluierung. Trainingsmanual. Bern: Huber.

Ries, H. (1970). Berufswahl in der modernen Industriegesellschaft. Bern: Huber.

Scheller, R. (1976). Psychologie der Berufswahl und der beruflichen Entwicklung. Stuttgart: Kohlhammer.

Seifert, K. H. (1977). Theorien der Berufswahl und der beruflichen Entwicklung. In K. H. Seifert (Hrsg.), Handbuch der Berufspsychologie (S. 173-279). Göttingen: Hogrefe.

Sesink, W. (2007). Bildung und Medium. Bildungstheoretische Spurensuche auf dem Felde der Medienpädagogik. In W. Sesink; M. Kerres \& H. Moser (Hrsg.), Jahrbuch Medienpädagogik 6: Medienpädagogik - Standortbestimmung einer erziehungswissenschaftichen Disziplin (S. 70-100). Wiesbaden: VS-Verlag.

Shannon, C. E. (1998). The Mathematical Theory of Communication. In C. E. Shannon $\&$ W. Weaver (eds.), The Mathematical Theory of Communication ( $3^{\text {rd }}$ ed., pp. 29125). Urbana \& Chicago: University of Illinois.

van den Bulck, J. \& Beullens, K. (2007). The Relationship between Docu Soap Exposure and Adolescents' Career Aspirations. European Journal of Communication, 22(3), 355-366.

Weaver, W. (1998). Some Recent Contributions to the Mathematical Theory of Communication. In C. E. Shannon \& W. Weaver (eds.), The Mathematical Theory of Communication ( $3^{\text {rd }}$ ed., pp. 1-28). Urbana \& Chicago: University of Illinois.

Weyer, C., Gehrau, V. \& Brüggemann, T. (2016). Der Einfluss von Medien auf die Entwicklung von Berufswünschen im Prozess der Berufsorientierung. Zeitschrift für Berufs- und Wirtschaftspädagogik, 112(1), 108-126. 



\title{
Inklusive Lernumgebungen mit digitalen Medien in Überbetrieblichen Berufsbildungsstätten der Bauindustrie
}

\author{
Susanne Korth, Svenja Noichl und Volker Rexing
}

\section{Ausgangslage}

Die Forderung nach inklusiver Bildung ist keineswegs neu und wird gleichermaßen international wie auch deutschlandweit intensiv diskutiert. In die internationale Diskussion eingeführt wurde der Begriff inclusive education bereits 1994 mit dem Salamanca Statement (United Nations Educational 1994), öffentlich breit thematisiert erst seit 2008 mit dem Übereinkommen der Vereinten Nationen über die Rechte von Menschen mit Behinderungen (häufig abgekürzt als Behindertenrechtskonvention BRK) (VN-BRK 2008; Hinz 2013). Seitdem dokumentiert sich die Forderung nach inklusiver Bildung zunehmend in reformierten Gesetzen und Verordnungen auf vielfältigen Handlungsebenen des Bildungssystems. Die Diskussion fokussiert dabei bisher weitgehend die Entwicklungen im allgemeinbildenden Schulbereich. Bezüge zu Fragen der Berufsbildung scheinen demgegenüber bislang eher vernachlässigt (Euler 2016, 27). Dabei fordert bereits das Berufsbildungsgesetz vom 23.03.2005 ( $\S$ $64 \mathrm{BBiG}$ ) ausdrücklich, dass behinderte Menschen in anerkannten Ausbildungsberufen ausgebildet werden sollen. Dies impliziert das Recht aller Jugendlichen mit Behinderungen, gemeinsam mit Nicht-Behinderten lernen zu dürfen.

In dem Kontext kann von einer gewissen Zahl von als lernbehindert anzunehmenden Auszubildenden ausgegangen werden, die in Ausbildungsberufe (auch) der Bauwirtschaft einmünden. Entscheidend ist in diesem Zusammenhang, dass es sich bei der Kategorie sonderpädagogischer Förderbedarf im Bereich Lernen um ein „Label“ auf Zeit handelt, das mit Verlassen der allgemeinbildenden Schule seine Gültigkeit verliert (z. B. Euler 2016, 34). Dadurch werden Jugendliche mit einer zuvor diagnostizierten Lernbehinderung in der groBen Gruppe der Jugendlichen ohne Schulabschluss nicht mehr von Lernenden ohne Behinderung unterscheidbar, obwohl mehr als die Hälfte aller Schulabgänger*innen ohne (Haupt-)Schulabschluss aus Förderschulen gekommen sind (Autorengruppe Bildungsberichterstattung 2014). Alle Schulabgänger*innen haben grundsätzlich die Möglichkeit, in das duale System der beruflichen Bildung einzutreten und eine berufliche Erstausbildung aufzunehmen (Arnold \& Münch 2000). 
Das Handwerk und die Bauindustrie dürften ein zunehmend gesteigertes Interesse daran haben, auch diesen Schüler*innen mit problematischen Lernvoraussetzungen die Möglichkeit einer erfolgreichen Ausbildung zu eröffnen, da aufgrund des demografischen Wandels ein stetig wachsender Bedarf an Fachkräften zu verzeichnen ist. Dieser Fachkräftemangel zeichnet sich in besonderem Maße für die Bauwirtschaft ab (DIHK 2011; hierzu auch Petsch, Norwig \& Nickolaus 2014). Für die Ausbildungsbetriebe dieses Wirtschaftszweigs können gerade Auszubildende mit einer ausgeprägten praktischen Begabung aufgrund des hohen Praxisanteils in der Ausbildung gewinnbringend sein. Für Menschen mit Lernbehinderungen könnte eine Ausbildung in der Bauwirtschaft, die über ein Erlernen im Handeln und Tun, bei dem über greifbare, sinnliche Erfahrungen berufsfachliche Kompetenzen erworben werden, ebenso einbringlich sein. Werden die derzeitigen Ausbildungsangebote noch um weitere unterstützende Förderangebote erweitert, so könnten auch Lernende mit defizitären Lesekompetenzen, Rechenstörungen und weiteren unter Lernbehinderungen subsummierten Lernstörungen durchaus einen qualifizierten Beruf erlernen und nachhaltig ausüben.

Für die Berufe der Bauwirtschaft haben in diesem Zusammenhang die Überbetrieblichen Berufsbildungsstätten (ÜBS) eine quantitativ und qualitativ besondere Bedeutung (z. B. Meyser 2010). Im dualen System der beruflichen Bildung ergänzen die überbetrieblichen Berufsbildungsstätten (ÜBS) als dritter Lernort bzw. Verbundpartner der Ausbildungsbetriebe die Berufsausbildung. Aufgrund von Spezialisierungen sind nicht alle KMU in der Lage, die in der Ausbildungsordnung geforderten Ausbildungsinhalte am Arbeitsplatz zu vermitteln (Schelten 2010, 75). Durch das ergänzende Ausbildungsangebot der ÜBS können Niveauunterschiede der betrieblichen Berufsausbildung ausgeglichen und der Transfer aktueller Techniken und Technologien in die Betriebe unterstützt werden (Arnold \& Münch 2000). Darüber hinaus bietet der Lernort ÜBS - im Unterschied zur Berufsschule und zum Ausbildungsbetrieb - die Möglichkeit der systematischen Verknüpfung von theoretischer Aneignung und praktischer Anwendung. In berufs(feld)nahen Anwendungskontexten können Lerngegenstände durch konkretes Tun erprobt werden.

Trotz der besonderen Relevanz der ÜBS hat das Thema Inklusion hier bislang vergleichsweise wenig Aufmerksamkeit erfahren (z. B. Köhlmann-Eckel 2015). Aus diesem Grund ist es ein zentrales Anliegen des in diesem Beitrag vorgestellten BMBF-Verbundprojekts Digitale Medien als Werkzeuge inklusiven Lernens in Überbetrieblichen Berufsbildungsstätten der Bauindustrie (MeinBerufBau), diesen Lernort verstärkt in den Blickpunkt inklusionsorientierter Bildungsangebote zu nehmen. Im Unterschied zum Lernen im Betrieb, das i. d. R. ökonomischen und arbeitsorganisatorischen Restriktionen unterliegt, bietet die Entwicklung inklusiver Lernumgebungen vor allem in den ÜBS größere Freiheitsgrade für die Gestaltung systematischer Lehr-Lernprozesse und Raum für gemeinsames Lernen (z. B. Euler 2015). Insbesondere für 
Auszubildende mit Lernbeeinträchtigungen und deren spezifische Unterstützungsbedarfe erscheint dieser Lernort folglich in besonderem Maße potenzialreich.

Im Folgenden werden nach einer kurzen Projektbeschreibung vor dem Hintergrund des entsprechenden Forschungs-/Entwicklungsdesigns erste Ergebnisse des insgesamt drei Jahre (bis 2021) laufenden Projekts vorgestellt, die folglich (insbesondere im Kontext des das Projekt leitenden Design-Based-Research-Ansatzes) exemplarischen und vorläufigen Charakter haben. Der Beitrag schließt mit einem Ausblick auf den weiteren Forschungs-/Entwicklungsprozess.

\section{Das Projekt „MeinBerufBau“}

Das BMBF-Verbundprojekt Digitale Medien als Werkzeuge inklusiven Lernens in Überbetrieblichen Berufsbildungsstätten der Bauindustrie (MeinBerufBau) lehnt sich an das Handlungsfeld „Gemeinsame Lernkonzepte für Menschen mit und ohne Behinderung“ der entsprechenden Bekanntmachung „Inklusion durch digitale Medien in der beruflichen Bildung“" (BMBF 2017) an. Im Rahmen dieses Handlungsfeldes sollen digitale Lernsettings entwickelt werden, die neuartige Formen für ein gemeinsames Lernen von Auszubildenden mit und ohne Lernbehinderung bereitstellen. Im Fokus steht dabei die Gestaltung barrierefreier Lern- und Bildungsprozesse, um Auszubildende beim Erlernen und langfristigen Ausüben einer beruflichen Tätigkeit zu unterstützen. Projektbeteiligte sind neben dem Lehr- und Forschungsgebiet Fachdidaktik Bautechnik der RWTH Aachen das Lehr- und Forschungsgebiet Informatik 9: Learning Technologies sowie das Berufsförderungswerk der Bauindustrie NRW, vertreten durch das Ausbildungszentrum der Bauindustrie Kerpen (ABZ Kerpen).

Im Rahmen des Projekts MeinBerufBau werden im Verständnis gemeinsamen Lernens grundsätzlich alle Lernenden adressiert, wobei der Schwerpunkt auf der Förderung vulnerabler Gruppen liegt (z. B. Werning \& LütjeKlose 2016; Lindmeier \& Lütje-Klose 2015). D. h., gleichwohl alle Auszubildenden einbezogen werden, sollen in besonderer Weise die Bedürfnisse von Auszubildenden mit einer Lernbehinderung in den Blick genommen werden. In diesem Bereich besteht aus Sicht der Betriebe der größte Unterstützungsbedarf (Galiläer \& Ufholz 2016; hierzu auch Enggruber \& Rützel 2016). Andere (z. B. körperliche) Formen von Behinderung sind hingegen aufgrund marktbedingter Selektionsmechanismen und Spezifika der primär handwerklichen Tätigkeiten des in diesem Vorhaben fokussierten Berufsfelds (Bautechnik) deutlich weniger relevant (Bach 2017). 
Verortet ist das Projekt in der berufsfeldbreiten Grundbildung (1. Ausbildungsjahr) der Berufe in der Bauwirtschaft. Diese stellt als Berufseingangsphase für Auszubildende einen bedeutsamen Schritt des Übergangs hin zu einer erfolgreichen Ausbildung dar. Im Konzept der Stufenausbildung werden bereits im 1. Ausbildungsjahr viele für die gesamte Ausbildung relevante (praktische und theoretische) Fachinhalte angelegt. Entsprechend der Breite des Berufsfeldes vereint die Grundbildung 16 verschiedene Berufe (BMWi 1999) des Ausbau-, Hochbau- und Tiefbaugewerbes, die im Rahmen einer dualen Ausbildung erlernt werden können. Insofern ist die entsprechende Ausbildungsordnung (ebd.) die inhaltliche Referenz für das Projekt, d.h., die Adressaten des Projekts lernen im Kontext realer Inhalte an den für die duale Ausbildung üblichen Lernorten und gemeinsam mit allen anderen Auszubildenden.

In dem übergeordneten Projektziel - der Förderung von Inklusion in der Ausbildung der Bauwirtschaft - verbinden sich drei miteinander korrespondierende Teilziele. Das erste Ziel umfasst das Identifizieren von kognitiven Lernbarrieren, welches einer der wesentlichen Schwerpunkte der ersten Projektphase ist. Mithilfe einer Anforderungsanalyse ausbildungsrelevanter Fachinhalte sowie einer auf diese fachspezifischen Anforderungen abzielenden Analyse, welche die individuellen Lernvoraussetzungen der Auszubildenden untersucht, sollen zunächst mögliche berufsfachliche Lernbarrieren bei Auszubildenden ermittelt werden. Als zweites Projektziel wurde die Entwicklung inklusionsorientierter digitaler Lernsettings konstituiert, welche Auszubildende beim Erlernen und langfristigen Ausüben einer beruflichen Tätigkeit unterstützen sollen. Diese Lernsettings bündeln sich in einer digitalen LernApp in Form einer Toolbox aus vielfältigen Hilfetools zu diversen Fachinhalten des Baubereichs, welche unter Berücksichtigung und auf Basis der vorher identifizierten Anforderungen und individuellen Lernpotentialen konzipiert und entwickelt wird. Der Zugriff auf die Lern-App erfolgt über mobile Endgeräte als Cross-Plattform-Entwicklung. Sie soll für jeden Auszubildenden eine persönliche Lernumgebung (Personal Learning Environment; kurz: PLE) vorhalten, die sich an den jeweiligen Bedürfnissen u. A. auf Basis von Nutzungsdaten in einem adaptiven Lernprozess orientiert. Die Pilotierung der Lernapplikation - als drittes Projektziel - findet ebenso im Rahmen der berufsfeldbreiten Grundbildung im ersten Ausbildungsjahr der Überbetrieblichen Berufsbildungsstätte (ÜBS) des beteiligten Projektpartners ABZ Kerpen statt. Dabei sollte die Lernapplikation neben dem traditionellen Material und den konventionellen Anleitungen durch das Lehrpersonal begleitend eingesetzt werden, da erwartungsgemäß hierdurch die Leistung von Lernenden positiv beeinflusst wird (Hillmayr, Reinhold, Ziernwald \& Reiss 2017). 


\section{Forschungs- und Entwicklungsdesign}

Die leitende Forschungs- und Entwicklungsmethode im Rahmen des Projekts MeinBerufBau folgt dem Design-Based Research (DBR). Grundlegend für den DBR-Ansatz ist das Ziel, die Entwicklung innovativer Lösungen für praktische Bildungsprobleme mit der Gewinnung wissenschaftlicher Erkenntnisse zu verknüpfen. Ausgangspunkt ist dabei die Frage, wie ein erstrebenswertes Bildungsziel (hier: Inklusion) in einem gegebenen Kontext (hier: die Ausbildungsrealität von Auszubildenden mit und ohne Lernbehinderungen) durch eine schrittweise zu elaborierende Innovation (hier: digitale Lernsettings) am besten zu erreichen ist (Abb. 1). In diesem Sinne ist es nicht nur das Anliegen des DBR, die bestehende Ausbildungsrealität zu untersuchen, sondern vor allem zukünftige Möglichkeiten inklusionsorientierter Ausbildung zu entwickeln. Der DBR-Ansatz unterscheidet sich von anderen Forschungsmethoden dadurch, dass prospektive und reflektierende Komponenten des Forschungsdesigns nicht voneinander getrennt sind (z. B. Cobb, Confrey, diSessa, Lehrer \& Schauble 2003).

Abb. 1: Design-Based Research (DBR)

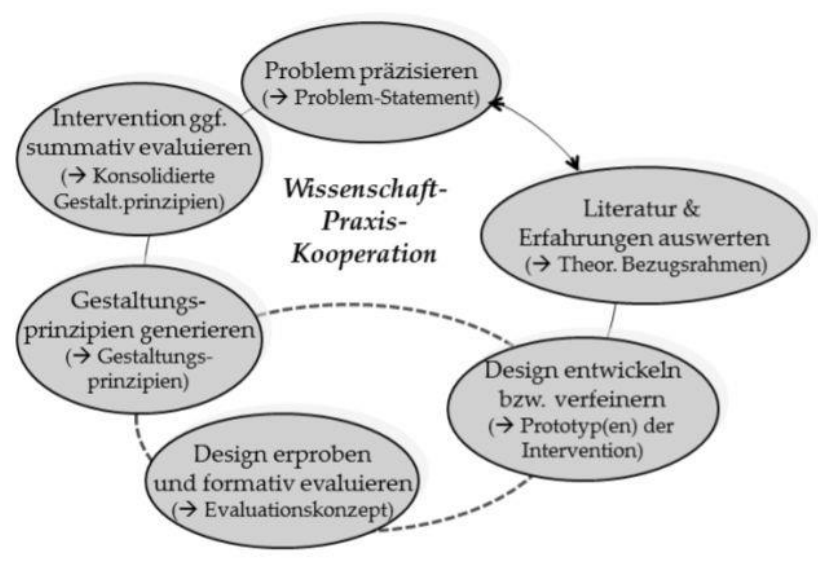

Quelle: Euler 2014, 20

Die Operationalisierung des DBR-Ansatzes im Projektkontext in fünf übergreifende Projektphasen zeigt Abbildung 2. Projektphase I fokussiert einen Kernaspekt des Projekts, die Identifikation von subjektiven berufsfachlichen Lernbarrieren. Dazu werden zunächst ausbildungsrelevante Lerngegenstände identifiziert. Darüber hinaus erfolgt eine auf individuelle Lernbeeinträchtigungen gerichtete differenzierte Beschreibung der Adressaten und deren (Lern-) 
Voraussetzungen. In dem Zusammenhang werden auch Rahmenbedingungen in der ÜBS zur Gestaltung von Lehr- und Lernprozessen (z. B. zum bisherigen Umgang mit heterogenen und teilweise defizitären Lernvoraussetzungen) erhoben.

Abb. 2: Arbeitsplan Projekt MeinBerufBau

\begin{tabular}{|c|c|c|}
\hline $\begin{array}{l}\text { Projekt- } \\
\text { phase }\end{array}$ & $\begin{array}{c}\text { Arbeits- } \\
\text { paket }\end{array}$ & Inhalt \\
\hline I & AP $1-6$ & Identifikation von Lernbarrieren \\
\hline II & AP 7-10 & $\begin{array}{l}\text { Entwicklung Prototyp } 1.0 \text { und Qualifizierung der Ausbilder- } \\
\text { *innen in einem iterativen kollaborativen Entwicklungs- } \\
\text { zyklus }\end{array}$ \\
\hline \multirow[t]{2}{*}{ III } & AP $11-15$ & Pilotphase I: \\
\hline & & $\begin{array}{l}\text { Erprobung der Lernapplikation und prozessbegleitende } \\
\text { Evaluierung, Anpassung und Optimierung der Lern- } \\
\text { applikation in Pilotphase sowie Durchführung von Work- } \\
\text { shops für betriebliches Ausbildungspersonal }\end{array}$ \\
\hline \multirow[t]{2}{*}{ IV } & AP $16-18$ & Pilotphase II: \\
\hline & & $\begin{array}{l}\text { Kontinuierliche technische und didaktisch-methodische } \\
\text { Optimierung und Erweiterung sowie Evaluierung der Lern- } \\
\text { applikation }\end{array}$ \\
\hline $\mathrm{V}$ & AP $19-21$ & $\begin{array}{l}\text { Abschlussevaluation sowie Veröffentlichung der Materia- } \\
\text { lien als Open Education Resources (OER) und der Lern- } \\
\text { applikation als Open Source Software (OSS) sowie } \\
\text { Implementierung der Projektergebnisse in die Stufen- } \\
\text { ausbildungsverordnung Bau }\end{array}$ \\
\hline
\end{tabular}

Quelle: eigene Darstellung

Um Lernbarrieren unter den vorhin genannten Grundannahmen identifizieren zu können, wurde in der ersten Projektphase eine komplexe Anforderungs- und Lernpotentialanalyse vorgenommen. Dabei erfolgte zunächst eine Klärung der berufsfachlichen Anforderungen über eine Dokumentenanalyse. Die Rahmenbedingungen am Lernort ÜBS (mit dem Schwerpunkt auf die Gestaltung von Lehr-/Lernprozessen) konnten in halbstrukturierten, leitfadengestützten Experteninterviews mit Ausbildern verschiedener Lehrgangsbereiche erhoben werden.

Bei der weiteren Diagnose kognitiver Lernbarrieren respektive Lernbeeinträchtigungen wurden verschiedene Instrumente und methodische Zugänge eingesetzt. Eine differenzierte Identifizierung von Lernbarrieren erfolgte u. a. 
aus den Daten eines Experten-Workshops mit Ausbildern am Lernort ÜBS. Zentral war vor dem Hintergrund dieses Erkenntnisinteresses aber insbesondere der Zugang über die Methode des Lauten Denkens (z. B. Knoblich \& Öllinger 2006). Dabei wird der/die Auszubildende aufgefordert, Gedanken bei der Bearbeitung der entsprechenden Aufgabe möglichst detailliert zu verbalisieren. Diese handlungsbegleitenden Verbalisierungen wurden transkribiert und inhaltsanalytisch ausgewertet. Die hier genutzte Aufgabensammlung setzte sich aus berufsfachlichen Aufgaben zusammen, die sich primär an den Ausbildungsrahmenplänen für die jeweiligen Berufsausbildungen im Hoch-, Tief- und Ausbaubau orientierten.

Die zweite Projektphase umfasste die iterative und kollaborative Entwicklung einer ersten Version der Toolbox. Diese folgte dem Adaptiven Software Development (ASD). Dabei wurden in einem iterativen Zyklus kontinuierlich neue Programmversionen entwickelt und in regelmäßigen Abständen systematisch formativ evaluiert. Neben der Softwareentwicklung werden auch Workshops zur Qualifizierung der Ausbildenden in der ÜBS konzeptioniert, die später die entwickelte Lernapplikation respektive die Toolbox mit den Auszubildenden erproben werden. Hier geht es insbesondere um technische Fragen in Bezug auf den Umgang und Einsatz der Toolbox in der spezifischen Gestaltung der überbetrieblichen Ausbildung. In diesem Zusammenhang werden u.a. Lehr- und Lernmaterialien sowie Guidelines für den technischen und didaktisch-methodischen Einsatz der Toolbox entwickelt. Der aktuelle Arbeitsstand stellt sich so dar, dass diese Phasen abgeschlossen sind und in Pilotphase I die Erprobung der Lernapplikationen in realen Lehr-Lernprozessen am Lernort ÜBS erfolgt.

\section{Ausgewählte Ergebnisse}

An dieser Stelle soll exemplarisch für den Arbeitstand auf den ersten Prototypen der Lernapp in MeinBerufBau eingegangen werden. Um beide Zielgruppen, Auszubildende und Ausbildende, darin zu unterstützen, autonom mit diesem System interagieren zu können, gibt es drei unterschiedliche Komponenten: das Aufgabenmodul, das Autorentool und die Hilfetools (s. Abb. 3). 
Abb. 3: Aufbau und Bestandteile der Lernapplikation (Prototyp 1.0)

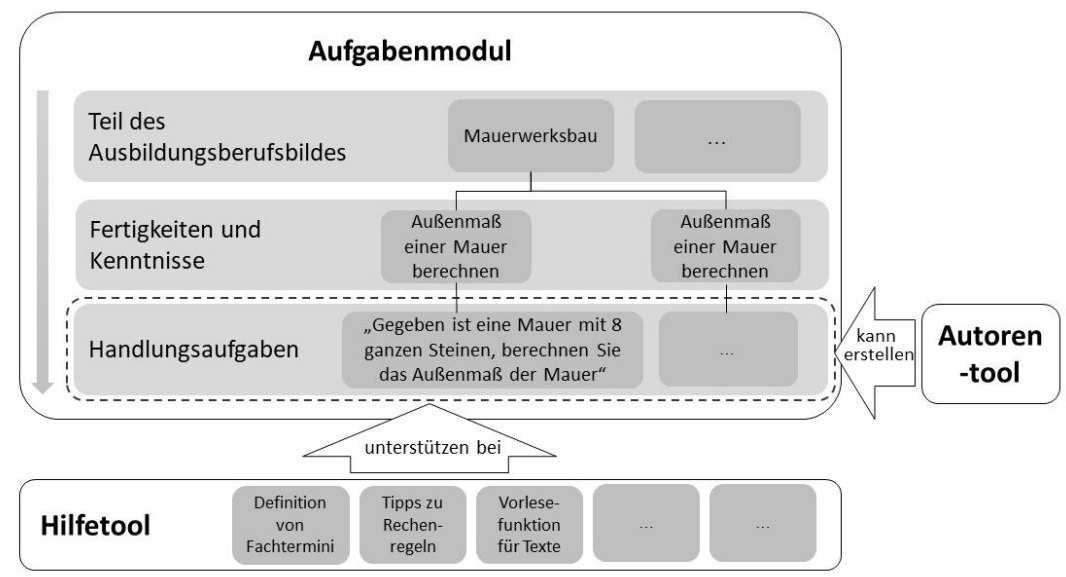

Quelle: In Anlehnung an Noichl, Korth \& Schroeder 2019, 5

Das hierarchisch aufgebaute Aufgabenmodul beinhaltet im Wesentlichen Handlungsaufgaben zu verschiedenen Fragestellungen aus dem Beton-/Stahlbetonbau, Holzbau, Kanalbau, Rohrleitungsbau, Straßenbau und Mauerwerksbau. Dabei kann zum jetzigen Entwicklungszeitpunkt der Anwender auf der obersten Ebene aus den sechs berufsfachlichen Schwerpunkten und der untergeordneten Ebene aus entsprechenden Fertigkeiten, Kenntnissen und Fähigkeiten eine Auswahl treffen. Die berufsfachlichen Schwerpunkte korrespondieren im Übrigen mit dem ÜBS-internen Curriculum am ABZ Kerpen und entsprechend mit den Teilen des Ausbildungsberufsbildes, die im Ausbildungsrahmenplan für die Berufsausbildung zum/zur Hochbaufacharbeiter*in, Ausbaufacharbeiter*in und Tiefbaufacharbeiter*in benannt sind (BMWI 1999).

So wurde im Prototyp 1.0 beispielsweise für den Teil des Ausbildungsberufsbildes „Herstellen von Baukörpern aus Steinen“ auf die zu vermittelnde berufliche Fertigkeit, Kenntnis bzw. Fähigkeit „Mauerwerk aus klein- oder mittelformatigen Steinen herstellen“ Bezug genommen (ebd., 1134). Hierzu finden Auszubildende nach Auswahl einer entsprechenden beruflichen Fertigkeit, Kenntnis oder Fähigkeit verschiedene konkrete Handlungssituationen vor, in die zu bearbeitende Handlungsaufgaben eingebettet sind. Den Auszubildenden wird im Aufgabenmodul jederzeit ermöglicht, gezielt für sie passende Aufgabenstellungen individuell nach ihren Bedürfnissen auszuwählen. 
Mit Hilfe eines Autorentools können, neben den bereits im Rahmen des Projekts implementierten Handlungsaufgaben, Ausbildende einfach und schnell eigene Aufgabenformulierungen generieren. So wird den standortspezifischen Ergänzungs- und Wahlcurricula sowie dem Aspekt der Nachhaltigkeit - als eine zentrale Zielsetzung des Projektvorhabens - Rechnung getragen. Ausbildende können die Lernapp mithilfe des Autorentools auch nach Projektbeendigung weiterverwenden und bedarfsorientiert ausbauen.

Dazu ist es notwendig, auch das Autorentool als ein möglichst barrierefreies Werkzeug zu entwickeln. Da davon auszugehen ist, dass die Zielgruppe (der Ausbildenden) größtenteils nicht über ausreichende informationstechnische Kompetenzen verfügt, um Webseiten oder mobile Applikationen selbstständig programmieren zu können, ermöglicht das Autorentool eine einfache Erstellung von Aufgaben über Templates.

Damit eine Aufgabe am Ende barrierefrei vom System dargestellt werden kann, sind in der Entwicklungsphase fundierte Kenntnisse über die vielfältigen Aspekte der barrierefreien Gestaltung einer Lernapp erforderlich. Da diese in den meisten Fällen bei den Ausbildern nicht vorhanden sind, unterstützt auch hier das Autorentool über Templates die Ausbildenden. Das Autorentool bietet dadurch beispielsweise eine einfache Einbettung von Videos mit Untertiteln. Ausbildende müssen lediglich Dateien und Texte über Pflichtfelder eingeben. Das System bildet die Inhalte innerhalb der Aufgabe entsprechend barrierefrei ab (s. Abb. 4).

Abb. 4: Template zum Einfügen von Videos im Autorentool

\begin{tabular}{|l|}
\hline Video mit Audiobeschreibung \\
Datei auswăhlen Keine ausgewăhlt \\
Video \\
Datei auswăhlen Keine ausgewählt \\
Alternativer Text \\
\hline Volltextalternative \\
\hline Untertitel \\
\hline Datei auswăhlen Keine ausgewăhlt \\
\hline
\end{tabular}

Quelle: Noichl, Korth und Schroeder 2019, 6

Ebenso leicht gestaltet sich die Einbindung von dreidimensionalen Animationen oder Abbildungen wie z. B. technischen Zeichnungen, welche für die Bewältigung von Aufgabenstellungen in der Bautechnik oftmals grundlegend 
sind. Diese Einbettungsmöglichkeiten sind ein wesentlicher Vorteil von mobilen Anwendungen gegenüber papierbasierten Aufgaben, da beispielsweise Videosequenzen zur Unterstützung des „Lernen am Modell“ nicht nur kognitive, sondern darüber hinaus auch erhöhte motivationale und emotionale Funktionen besitzen können.

Videos und Animationen sind in der Lernapp über die Funktion Hilfetools integrierbar. Als Interaktionswerkzeuge ermöglichen diese (z. B. durch die Stopp- oder Wiederholungsfunktion) den Auszubildenden, ihr Lerntempo selbst bestimmen können. Sie leisten somit einen Beitrag zum autonomen, individuellen Lernen. Entsprechend der Diversität der vorhandenen Lernpotentiale und der Variationsbreite von identifizierten Lernbarrieren sind bereits im ersten Prototyp neun Hilfetools entwickelt worden. Deren Konzipierung stützt sich, wie auch die der Lerninhalte und Aufgabenstellungen, auf die Analyseergebnisse der ersten Projektphase. Diese sind in der Lernapplikation für den/die User/-in aufgrund der Auswertung der individuellen Nutzungsdaten automatisiert zugeschaltet oder können vom Anwender gezielt ausgewählt werden. Dem übergeordneten Ziel des Projekts folgend, Inklusion in der Ausbildung zu fördern, sollen besonders mithilfe der Hilfetools die Auszubildenden in der Überwindung individueller Lernbarrieren und damit in einer erfolgreichen und autonomen Aufgabenbewältigung unterstützt werden. Dies gilt insbesondere auch für lernbeeinträchtigte Auszubildende in der Bauwirtschaft. An zwei konkreten Lernbarrieren soll exemplarisch illustriert werden, welches Potential digitale Medien (in dem Fall die Lernapp MeinBerufBau) besitzen.

Eine der identifizierten Lernbarrieren ist beispielsweise die „Kenntnis von Fachtermini“", welche beim Verständnis der textbasierten Aufgabenstellung auftreten und die Auszubildende bereits zu Bearbeitungsbeginn behindern kann. Um zu verhindern, dass eine Aufgabenstellung aufgrund von fehlender oder fehlerbehafteter Kenntnis von Fachtermini nicht verstanden wird, können die Auszubildenden daher jederzeit auf ein hinterlegtes Glossar zugreifen.

In Abbildung 5 ist dieses Hilfetool am Beispiel einer Aufgabenstellung zum Mauerwerksbau aus Sicht der Auszubildenden dargestellt. Die punktierte Linie unterhalb eines Wortes oder einer Wortkombination signalisiert die Abrufbarkeit der entsprechenden Begriffsdefinition. Bei Auswahl des Begriffs öffnet sich ein Dialogfeld mit der Definition des markierten Worts bzw. der Wortkombination. Begriffe und deren Definitionen können von den Ausbildenden im Autorentool modifiziert oder neu angelegt werden. Die Definition kann sowohl textuell als auch bildlich erfolgen. Es besteht zudem die Möglichkeit, ähnliche Begriffe zusammenzuführen und alternative Schreibweisen zuzulassen. Zukünftig sollen auch die Auszubildenden in ihrer persönlichen Lernumgebung selbstständig Begriffe anlegen können, z. B. in einer alternativen Sprache neben der deutschen Standardsprache. 
Abb. 5: Aufgabe, Mauerlänge Binderverband ' und Hilfetool ,Glossar '
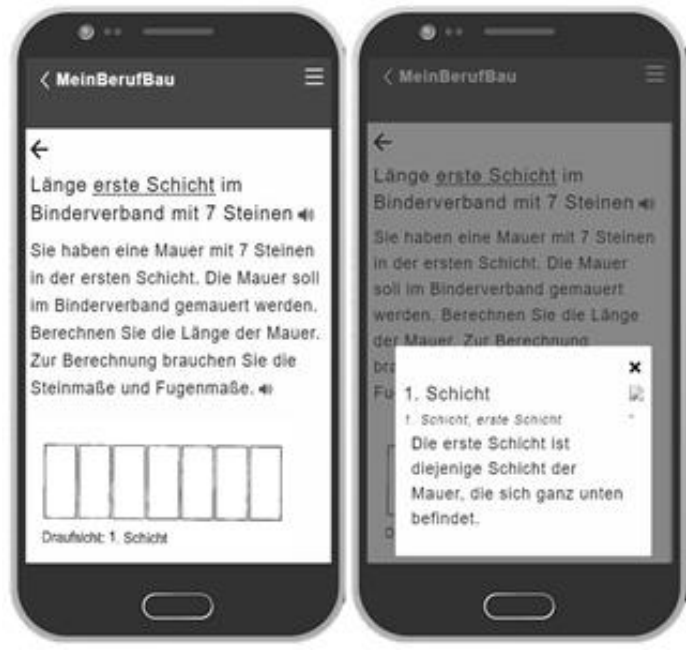

Quelle: Noichl, Korth \& Schroeder 2019, 6

Zur weiteren Förderung des Verständnisses der Aufgabenstellung wurde ein Hilfetool entwickelt, welches an der identifizierten Lernbarriere „Verständnis der Aufgabenstellung" anknüpft. So zeigte sich beispielsweise in den Prozessanalysen zur Aufgabenbearbeitung der Auszubildenden, dass diese bereits zu Beginn ungeeignete Lösungsansätze generieren. Ein Grund liegt u.a. darin, dass die Fragestellung nicht richtig erfasst wurde. Automatisierte Leitfragen derzeit in Form eines Multiple-Choice-Verfahrens - helfen zu Beginn einer Lernsequenz zu überprüfen, ob der/die User*in die Kernaussage der Aufgabenstellung nachvollziehen kann. Diese Kontrolle dient einerseits der Weiterentwicklung des Systems in Form von verwertbaren Nutzerdaten und andererseits den Auszubildenden zur Reflexion und ggf. Modifikation ihrer geplanten Vorgehensweise durch ein vom System erzeugtes Feedback. Dieses Feedback wird mithilfe von Learning Analytics auf die User*innen (entsprechend des aktuellen Lernfortschritts) individualisiert, als einfache binäre Aussage (Richtig-Falsch) oder in elaborierterer Form (z. B. ergänzende Fachinformationen oder Abbildungen) ausgegeben. So können Auszubildende, die vom System als zusätzlich förderungsbedürftig erkannt werden, in ihrem Verständnis der spezifischen Aufgabenstellung unterstützt werden. 


\section{Ausblick}

In Planung ist die Möglichkeit zur kollaborativen Zusammenarbeit mit anderen Auszubildenden durch ein weiteres Hilfetool, bei dem sich die Auszubildenden über die Lernapp vernetzen und gemeinsam am Lerngegenstand arbeiten können. Dies dient - so die Vorstellung der Projektbeteiligten - einer weiteren Lernförderung, besonders auch der (im engeren Sinne) lernbehinderten Auszubildenden. Gestützt wird dies z. B. durch eine empirische Metaanalyse zur Wirksamkeit digitaler Medien und deren Lernförderung im technisch-naturwissenschaftlichen Unterricht der Sekundarstufe II (Hillmayr, Reinhold, Ziernwald \& Reiss 2017). Hier zeigte sich deutlich, dass bei sozialer Unterstützung durch Mitschüler*innen (vor allem in Lerntandems) eine lernförderliche Wirkung von digitalen Medien nachweisbar ist. Der kommunikative Austausch über den Lerngegenstand und die vorhandenen Probleme beim Bearbeiten der Handlungsaufgaben bieten hier den Auszubildenden Raum, ihre heterogenen Wissensvoraussetzungen zum jeweiligen Lerngegenstand zu nutzen, um individuelle Hürden zu überwinden (ebd.).

Zum jetzigen Zeitpunkt wurde primär der berufsfachliche Schwerpunkt „Mauerwerksbau“ in die Applikation integriert. Neben der Weiterentwicklung dieses Schwerpunkts auf Basis von Evaluationsdaten wird in einem parallelen Prozess ein alternativer Aufbau der Lernapp im Bereich „Straßenbau“ entwickelt, der sich an dem Ablauf einer ,vollständigen Handlung“ orientiert. Projektaufgabe ist hier die Erstellung eines Gehwegabschnittes mit Pflastersteinen und Tiefenbordsteinen aus Beton. Diese lehnt sich an die Aufgabenstellung der „Unterlagen für Auszubildende“ an, die vom Bundesinstituts für Berufsbildung (BIBB) herausgegeben wurden und entspricht einer konkreten Aufgabenstellung der Ausbildungsrealität an der ÜBS (Meyser \& Uhe 2012).

Aktuell befindet sich das Projekt in der dritten Projektphase (Pilotphase I; s. hierzu Abb. 2), in der die Pilotierung der bis hierhin entwickelten Lernapplikationen mit ca. 100 Auszubildenden erfolgt. Dabei wird der Einsatz der Lernapplikationen bzw. der Toolbox insbesondere in technischer Hinsicht evaluiert und diese sukzessive bedarfsorientiert optimiert. Hierzu werden kontinuierlich über den gesamten Zeitraum die (anonymisierten) Userdaten ausgewertet, um Aussagen zur Nutzungshäufigkeit, Nutzungsart bzw. -dauer seitens der Auszubildenden treffen zu können. Diese Userdaten werden für die Ermöglichung adaptiver Lernprozesse verwendet. Auf Basis der Nutzerdaten werden so beispielsweise individuell angepasste Lernhilfen (z. B. alternative Darstellung von Lerninhalten, Rechenschritthilfen) bereitgestellt und die Aufgaben auf die jeweiligen Nutzer angepasst. Ferner tragen die Daten zur Software-Optimierung bei und geben Hinweise z. B. auf Interaktionsschwierigkeiten oder werden für eine adaptive Anpassung der grafischen Benutzeroberfläche (z. B. Schriftgröße) verwendet. 
Mit Abschluss der Erprobungsphase ist die Durchführung eines Workshops verbunden, in dem Zwischenergebnisse und Best-Practice-Beispiele den beteiligten Akteuren vorgestellt werden. Parallel erfolgt ÜBS-intern eine Verbreitung der Zwischenergebnisse über interne Workshops/Fortbildungsveranstaltungen für betriebliches Ausbildungspersonal i. S. v. Transfer und Lernortkooperation. Des Weiteren erfolgt eine Dissemination der Zwischenergebnisse an 330 Mitgliedsunternehmen der Bauindustrie NRW.

\section{Literatur}

Arnold, R. \& Münch, J. (2000). 120 Fragen und Antworten zum Dualen System der deutschen Berufsausbildung. Baltmannsweiler.

Autorengruppe Bildungsberichterstattung (2014). Bildung in Deutschland 2014. Ein indikatorengestützter Bericht mit einer Analyse zur Bildung von Menschen mit Behinderungen. Bielefeld.

Bach, A. (2017). Inklusion im Dualen System der gewerblich-technischen Berufsbildung. Empirische Vorstudie im Berufsfeld Bautechnik. Berufsbildung, 166(15), $15-17$.

Bundesministerium für Bildung und Forschung (BMBF) (2017). Bekanntmachung der Richtlinie zur Förderung von "Inklusion durch digitale Medien in der beruflichen Bildung". Online: https://www.bmbf.de/foerderungen/bekanntmachung-1317. html (05.10.2017).

Bundesministerium für Wirtschaft und Energie (BMWI) (1999). Verordnung über die Berufsausbildung in der Bauwirtschaft. Online: http://www.gesetze-im-internet.de/bundesrecht/bauwiausbv_1999/gesamt.pdf (05.11.2019) bzw. Bundesgesetzblatt (BGBL), 1999 I, Nr. 23.

Cobb, P., Confrey, J., diSessa, A., Lehrer, R. \& Schauble, L. (2003). Design Experiments in Educational Research. Educational Researcher, 32(1), 9-13.

Deutscher Industrie- und Handelskammertag (DIHK) (2011). DIHK-Mittelstandsreport. Berlin.

Enggruber, R. \& Rützel, J. (2016). Berufsausbildung junger Menschen mit Behinderungen. Ausgewählte Ergebnisse einer repräsentativen Befragung von Ausbildungsbetrieben. In A. Zoyke \& K. Vollmer (Hrsg.), Inklusion in der Berufsbildung: Befunde - Konzepte - Diskussionen (S. 79-98). Bielefeld: W. Bertelsmann Verlag.

Euler, D. (2014). Design-Research - a paradigm under development. In D. Euler \& P. F. E. Sloane (Hrsg.), Design-Based Research (S. 15-44). Stuttgart: Franz Steiner Verlag.

Euler, D. (2015). Lernorte in der Berufsausbildung zwischen Potential und Realität. Berufsbildung in Wissenschaft und Praxis, 62(109/119), 6-9.

Euler, D. (2016). Inklusion in der Berufsausbildung. Bekenntnisse - Erkenntnisse Herausforderungen - Konsequenzen. In A. Zoyke \& K. Vollmer (Hrsg.), Inklusion in der Berufsbildung: Befunde - Konzepte - Diskussionen (S. 27-42). Bielefeld: W. Bertelsmann Verlag. 
Galiläer, L. \& Ufholz, B. (2016). Ausbildung besonders förderbedürftiger Jugendlicher in und mit Betrieben. Erfahrungen aus dem Projekt TrialNet. In A. Zoyke \& K. Vollmer (Hrsg.), Inklusion in der Berufsbildung: Befunde - Konzepte - Diskussionen (S. 99-122). Bielefeld: W. Bertelsmann Verlag.

Hillmayr, D., Reinhold, F. Ziernwald, L. \& Reiss, K. (2017). Digitale Medien im mathematisch-naturwissenschaftlichen Unterricht der Sekundarstufe. Einsatzmöglichkeiten, Umsetzung und Wirksamkeit. Münster: Waxmann.

Hinz, A. (2013). Inklusion - von der Unkenntnis zur Unkenntlichkeit!? - Kritische Anmerkungen zu einem Jahrzehnt Diskurs über schulische Inklusion in Deutschland. Zeitschrift für Inklusion. Online: http://www.inklusion-online.net/index.php/inklusion-online/article/view/26/26 (07.08.2017).

Knoblich, G. \& Öllinger, M. (2006). Die Methode des lauten Denkens. In J. Funke \& P. A. Frensch (Hrsg.), Handbuch Allgemeine Psychologie: Kognition (S. 691696). Göttingen: Hogrefe.

Köhlmann-Eckel, C. (2015). Vielfältige Zielgruppen. Ein Lernort. Berufsbildung in Wissenschaft und Praxis, 62(109/119), 18-21.

Lindmeier, C. \& Lütje-Klose, B. (2015). Inklusion als Querschnittsaufgabe in der Erziehungswissenschaft. Erziehungswissenschaft, 26(51), 7-16.

Meyser, J. (2010). Berufsbildung in der Bauwirtschaft. In G. Syben (Hrsg.), Die Vision einer lernenden Branche im Leitbild Bauwirtschaft (S. 55-74). Berlin: Edition Sigma.

Meyser, J. \& Uhe, E. (2012). Handelnd Lernen in der Bauwirtschaft. Projektaufgaben Straßenbauer/in, Unterlagen für Ausbilder. Konstanz: Christiani.

Noichl, S., Korth, S. \& Schroeder, U. (2019). Mobile barrierefreie Lernanwendungen in der Bauindustrie. Mensch und Computer 2019 - Workshopband. Bonn: Gesellschaft für Informatik e.V. Online: https://dl.gi.de/bitstream/handle/20.500.12116/ 25191/422.pdf?sequence=1\&isAllowed=y (30.06.2020).

Petsch, C., Norwig, K. \& Nickolaus, R. (2014). Kompetenzförderung leistungsschwächerer Jugendlicher in der beruflichen Bildung - Förderansätze und ihre Effekte. Zeitschrift für Erziehungswissenschaft, 17 (Sonderheft 22), 81-101.

Schelten, A. (2010). Einführung in die Berufspädagogik. Stuttgart: Franz Steiner.

United Nations Educational, Scientific and Cultural Organization, Ministry of Education and Science Spain (1994). The Salamanca Statement and Framework for Action on Special Needs Education. Online: https://www.right-to-education.org/sites/right-to-education.org/files/resource-attachments/Salamanca_Statement_1994 .pdf (05.10.2017).

Werning, R. \& Lütje-Klose, B. (2016). Einführung in die Pädagogik bei Lernbeeinträchtigungen. München, Basel: Ernst Reinhardt Verlag (UTB). 


\title{
Die Bedeutung der Digitalisierung in der Neuausrichtung der pflegerischen Ausbildung - Herausforderungen für die berufliche Pflege im Kontext der Fachkräftesicherung
}

\author{
Jutta Mohr, Isabelle Riedlinger und Karin Reiber
}

\section{Aktuelle Megatrends in der beruflichen Domäne Pflege}

Die Domäne der beruflichen Pflege ist derzeit einem starken Wandel unterworfen. Branchenspezifische und intraprofessionelle Entwicklungen, wie der aktuelle Fachkräftemangel und die Neuausrichtung der beruflichen Ausbildung, stehen der Digitalisierung von Alltags- und Berufswelt gegenüber. In der aktuellen Statistik der Bundesagentur für Arbeit (BA) wird für die Gesundheits- und Krankenpflege in fast allen Bundesländern für die Altenpflege flächendeckend ein bereits manifester Fachkräftemangel identifiziert. Ein Fachkräftemangel liegt dann vor, ,wenn es im Verhältnis zur Arbeitsnachfrage (Stellenangebote) zu wenige passend qualifizierte Arbeitskräfte und zu wenige den Anforderungen entsprechend qualifizierbare Arbeitskräfte gibt" (BA 2018, 5). Dies lässt sich für die berufliche Pflege in nahezu allen Bereichen konstatieren; bundesweit lag die Arbeitslosenquote für die Altenpflege im Jahr 2019 bei $0,9 \%$ und für die Gesundheits- und Krankenpflege bei 0,6\% (BA 2018). Da nur ein Teil der offenen Stellen der BA gemeldet werden, gehen Expert*innen davon aus, dass sich die Situation insgesamt noch dramatischer darstellt, als sich dies an den statistischen Daten der BA ablesen lässt (Isfort \& Gesserich 2019). Aufgrund der demografischen Entwicklung ist zukünftig mit steigenden Bedarfen zu rechnen, die sowohl die quantitative Ausstattung (bspw. Flake et al. 2018) als auch die qualitative Ausgestaltung (bspw. Robert Koch-Institut 2015) beruflicher Pflege betreffen. Notwendig wird hierbei die Diskussion des Stellenwerts der Unterstützung durch digitale Technologien und deren Ansatzpunkte. Aktuell liegt die Gesundheitswirtschaft mit Digitalisierungsanteilen von etwas über $2 \%$ sowohl national als auch international im Vergleich der Wirtschaftsbereiche am unteren Ende (Vereinigung der Bayerischen Wirtschaft e. V. 2017). Mit den Potenzialen der Digitalisierung verbinden sich u. a. die Erwartung und Hoffnung, durch damit verbundene Arbeitserleichterungen den Pflegeberuf insgesamt attraktiver zu gestalten und zugleich die Berufsverweildauer zu erhöhen (Merda et al. 2017). Neben diesen hohen Erwartungen an die Potenziale der Digitalisierung treten zugleich aber 
auch erhebliche Befürchtungen hinsichtlich datenschutzrechtlicher und berufsethischer Fragen der pflegerischen Versorgung auf (Rösler et al. 2018). In beiden Fällen ist Digitalisierung häufig eine Chiffre für höchst unterschiedliche Anwendungen und Ausmaße von technisch-digitalen Unterstützungslösungen, über deren Reichweite zugleich auch eine große Unkenntnis herrscht (Buhtz et al. 2018).

Im Zusammenhang des aktuellen und zukünftigen Fachkräftebedarfs und der Erwartungen an Digitalisierungspotenziale in der beruflichen Pflege ist die 2020 startende Ausbildungsreform zu betrachten, die die Ausbildung in den Pflegeberufen grundlegend umstrukturiert. Um den Pflegeberuf und die Pflegeausbildung an die Veränderungen der Versorgungsstrukturen und anforderungen und die Fortschritte in der Pflegewissenschaft und den Bezugswissenschaften anzupassen, wurde nach längeren fachpolitischen Diskussions- und Aushandlungsprozessen ein neues Pflegeberufegesetz (PflBG 2017) erlassen. Dieses sieht u. a. vor, die bisher nach der Lebensphase der Klient*innen und Patient*innen differenzierten Pflegeberufe (Altenpflege, Gesundheits- und Krankenpflege, Gesundheits- und Kinderkrankenpflege) partiell $\mathrm{zu}$ einem einheitlichen Berufsprofil zu integrieren. Nach zwei generalistisch angelegten Ausbildungsjahren können sich die Auszubildenden nun entscheiden, ob sie das dritte Jahr mit einer Spezialisierung in der Altenoder Gesundheits- und Kinderkrankenpflege und der entsprechenden Berufsbezeichnung als Abschluss fortsetzen möchten oder ob sie ihre Ausbildung konsequent generalistisch mit dem Abschluss Pflegefachfrau/mann beenden. Ergänzt wird die berufsschulische Ausbildung nach dem neuen Pflegeberufegesetz durch die Möglichkeit einer hochschulischen Ausbildung zur Pflegefachfrau bzw. zum Pflegefachmann (PflBG 2017). Wenn in diesem Beitrag von beruflicher Pflege gesprochen wird, sind alle drei Fachbereiche (Altenpflege, Gesundheits- und Krankenpflege, Gesundheits- und Kinderkrankenpflege) gemeint. Im Kontext des Fachkräfteengpasses in der Pflege lässt sich konstatieren, dass der Ausbildung als wichtigster Säule der Fachkraftgewinnung (Fehst 2016) insbesondere hinsichtlich der hier schon anzubahnenden Prozesse der Berufsidentifikation und -bindung (Gerhardt \& Kanzog 2017) eine prominente Rolle zukommt. Wie sich nun die aus professionstheoretischen und versorgungspraktischen Gründen initiierte Ausbildungsreform darauf auswirkt, ist im Moment noch offen. Eine digitale Transformation lässt sich, neben Arbeits- und Geschäftsprozessen (Daum 2017), auch für die Bildungsprozesse der Gesundheitsberufe konstatieren (Kuhn et al. 2019). Vor dem Hintergrund des hohen Fachkräftebedarfs in der Pflege, hinsichtlich der benötigten digitalen Kompetenzen (PricewaterhouseCoopers 2016) und mit Blick auf eine Attraktivitätssteigerung des Berufs verbinden sich auch mit der Ausbildungsreform vielfältige und unterschiedliche, teilweise auch gegenläufige Erwartungen. 
Ziel dieses Beitrags ist es, im Hinblick auf die Erwartungshaltungen, die an die Digitalisierung beruflicher Pflege gestellt werden, den aktuellen Stand der Digitalisierung in der Praxis im Kontext der Fachkräftesicherung und die Ausgestaltung der generalistischen Pflegeausbildung hinsichtlich des Stellenwerts der Digitalisierung zu beleuchten, um hieraus Implikationen für die weitere Gestaltung sowohl des Digitalisierungsprozesses als auch dessen Verankerung in der Pflegeausbildung zu formulieren. Der Beitrag stellt zunächst die Rolle und Reichweite der Digitalisierung in der Pflege vor, um dann anhand empirischer Daten Erfahrungen mit und Erwartungen an technisch-digitale Unterstützung darzulegen. Auf der Basis dieses Anwendungsbezugs werden in einem weiteren Schritt die Ausbildungs- und Prüfungsverordnung des neuen Pflegeberufegesetzes wie auch die Rahmenlehr- und Rahmenausbildungspläne daraufhin befragt, welcher Stellenwert dem Thema Digitalisierung darin zukommt. Abschließend wird erörtert, wie die Nutzung und Anwendung digitaler Technik an das im Pflegeberufegesetz angelegte Berufsprofil anknüpfen und sich im Rahmen von Ausbildungspraxis und im Kontext der pflegerischen Versorgung berufsadäquat weiter entwickeln lassen.

\section{Die Rolle der Digitalisierung in der Pflege}

„Digitalisierung“ als wichtigste technologische Veränderung dieser Zeit bezeichnet primär die Integration digitaler Technologien in verschiedene Arbeitsprozesse (Lauterbach \& Hörner 2019). In personalen, sozialen Dienstleistungen, zu welchen berufliche Pflege gehört, geschieht Arbeit durch situationsund kontextabhängige Interaktion am und gemeinsam mit dem Menschen (Böhle \& Glaser 2006). Dadurch unterscheiden sich auch die Möglichkeiten der Digitalisierung von Arbeitsprozessen grundlegend von anderen Branchen (Pöser \& Bleses 2018) und weisen diesbezüglich ein geringeres Digitalisierungspotenzial auf (Daum 2017). Digitalisierung ist zunächst ein Sammelbegriff für diverse Anwendungen, Tools und Formate mit unterschiedlicher Reichweite, deren Einsatz in den verschiedenen Pflegesettings unterschiedlich ausgeprägt ist. Deshalb ist zunächst danach zu differenzieren, für welche Arbeitsschritte bzw. -anteile digitale Unterstützung genutzt wird und in welchem Maße berufliche Tätigkeiten digital unterstützt, mithilfe digitaler Technologie ergänzt und erweitert oder (teilweise) substituiert werden. Bislang liegt für die Domäne der Pflege keine konsentierte Definition bzw. trennscharfe Klassifikation digitaler Technologien vor. Beispielsweise werden digitale Technologien nach Gestaltungsfeldern in Informations- und Kommunikationstechnologien, intelligente und vernetzte Robotik und Technik und vernetzte Hilfs- und 
Monitoringsysteme klassifiziert (Daum 2017). In diesem Beitrag wird Digitalisierung anhand ihrer Einsatzfelder mit direktem Bezug zum pflegerischen Handeln in vier Bereiche systematisiert (Rösler et al. 2018):

1. Software für die Planung, Steuerung und Dokumentation pflegerischen Handelns;

2. Technische Assistenzsysteme, die den zu pflegenden Menschen und/oder die beruflich Pflegenden unterstützen;

3. Telecare/-nursing zur Unterstützung intra- und interprofessioneller Abstimmungs- und Entscheidungsprozesse sowie zur Kommunikation zwischen Personal und Nutzer*innen im Kontext der medizinisch-pflegerischen Versorgung;

4. Robotik zur Entlastung von zu pflegenden Menschen und/oder beruflich Pflegender sowie zur Ergänzung des pflegerischen Handelns.

Diese vier Einsatzfelder unterscheiden sich grundsätzlich in Entwicklungsstand und Verbreitung. Nachfolgend werden die verschiedenen Anwendungsgebiete kurz vorgestellt und der aktuelle Einsatz in der Pflege beschrieben. Zur Anwendung in der Praxis liegen insgesamt wenige Daten vor. Die nachfolgenden Angaben beziehen sich meist auf eine Umfrage der Berufsgenossenschaft für Gesundheitsdienst und Wohlfahrtspflege (BGW) unter 576 Pflegekräften im Jahr 2017 (Merda et al. 2017).

Software für die Planung, Steuerung und Dokumentation

Am weitesten verbreitet ist mittlerweile die digitale Dokumentation von Pflegeleistungen mithilfe digitaler Unterstützung in Form von PCs oder mobilen Endgeräten und passender Software. Diese Dokumentation kann eingebunden sein in ein erweitertes elektronisches Klient*innen-Dossier, das neben den pflegespezifischen Besonderheiten ergänzende gesundheitsbezogene Daten z. B. zu medizinischen Befunden, Diagnosen und Therapien sowie begleitender therapeutischer Maßnahmen enthält. Meist beinhaltet die elektronische Pflegedokumentation Schnittstellen zur Planung, Verwaltung und Abrechnung von Pflegeleistungen bzw. ist in solche Softwaresysteme eingebunden (Rösler et al. 2018). Hier handelt es sich also um die digitale Unterstützung von pflegeunterstützenden Verwaltungs- und Dokumentationsanteilen pflegeberuflichen Handelns. Wenn von Digitalisierung in der Pflege die Rede ist, wird meist dieser Bereich der elektronischen Pflegedokumentation gemeint. Das Bewusstsein für digitale Unterstützungsprozesse ist in diesem Bereich am meisten ausgeprägt. Während die digitale Dokumentation zunächst Einzug in die Krankenhäuser hielt - im Jahr 2014 hatten ca. zwei Drittel der Krankenhäuser mit der Umsetzung zumindest begonnen (Hübner et al. 2015) - zeigen neuere Er- 
hebungen, dass der Bekanntheitsgrad der digitalen Dokumentation mittlerweile in allen Pflegesektoren hoch ist und in der stationären Altenpflege und ambulanten Pflege den Krankenhaussektor bereits überholt hat (Merda et al. 2017).

\section{Technische Assistenzsysteme}

Technische Assistenzsysteme sollen besonders ältere und gesundheitlich beeinträchtige Personen in ihrer Selbständigkeit im Alltag unterstützen. Dabei geht es um Systeme, die alltägliche Aktivitäten messen und melden, wenn diese von der Norm abweichen. Dies können beispielsweise Softwarelösungen zur Lokalisierung von Personen mit Weglauftendenz oder intelligente Frühwarnsysteme, die (potenzielle) Sturzereignisse anzeigen, sein (Rösler et al. 2018). Es handelt sich dabei um Beispiele für digitale Unterstützungen, die einen indirekten Bezug zu Klient*innen haben und deshalb näher am pflegerischen Handeln verortet sind. Das bedeutet, dass die Pflegefachkräfte mittels dieser Systeme Informationen bzw. Warnmeldungen erhalten, auf die sie dann entsprechend reagieren können. Die digitale Unterstützung ist der Indikator, der eine pflegerische Intervention auslöst. Das pflegeberufliche Handeln ist somit eine Reaktion auf den digitalen Impuls, bleibt aber im Kern davon unberührt. Dieser Markt lässt sich nicht generell quantifizieren, da er stark wächst und sich viele Produkte noch in der Entwicklungsphase befinden. Am weitesten verbreitet sind technische Assistenzsysteme in der häuslichen pflegerischen Unterstützung mit 48\% (Merda et al. 2017).

\section{Telecare/-nursing}

Unter der Bezeichnung „Telecare“ bzw. „eCare“ firmieren digitale Lösungen zur Diagnostik und Beratung über räumliche Distanzen hinweg. Digitalisierung wird hier für den virtuellen Kontakt von Pflegefachperson und Patient*in genutzt, wenn ein realer Austausch aus räumlichen Gründen nicht möglich ist oder aber deren Realisierungsaufwand angesichts der Interventionsdauer nicht gerechtfertigt wäre (Rösler et al. 2018). Wie in der Telemedizin können solche Systeme in der Beratung über Distanzen hinweg genutzt werden. Im Gegensatz zu anderen Ländern, wie bspw. Australien und Kanada, wird Telecare in Deutschland überwiegend in Modellprojekten eingesetzt (Koehler et al. 2018). Nach der Nutzung von Telemedizin bzw. Telecare gefragt, gaben $27 \%$ der Befragten an, diese in ihrem Arbeitsalltag zu nutzen (Merda et al. 2017).

\section{Robotik}

Eine deutlich weiterreichende technisch-digitale Unterstützung sind RobotikAnwendungen. Hier reicht die Bandbreite der Anwendungen von haushaltsnahen pflegeergänzenden Technologien über emotionale Robotik bis hin zu belastungsreduzierenden und assistiven technischen Lösungen (Rösler et al. 
2018). Diese Form der Technisierung und Digitalisierung ist unterstützend als Servicerobotik oder direkt eingebunden in das pflegerische Handeln, bspw. beim Einsatz von Umsetz- und Hebehilfen bei körperlich anstrengenden Tätigkeiten (z. B. die Lagerung und der Positionswechsel von Patient*innen bzw. Bewohner*innen) im Einsatz oder ergänzt das Pflegehandeln wie im Falle der Emotionsrobotik. Auch der Einsatz von Robotik findet derzeit meist im Rahmen von Modellprojekten statt. Im Arbeitsalltag kommen bei $21 \%$ der Befragten Technologien zum Einsatz, die dem Bereich der Robotik zugeordnet werden können (Merda et al. 2017).

Die dargestellte Bandbreite an Einsatzmöglichkeiten digitaler Tools und technischer Lösungen verdeutlicht den eingangs konstatierten Befund, dass mit dem meist unscharf verwendeten Oberbegriff „Digitalisierung“ ebenso hohe Erwartungen (z. B. an Arbeitserleichterung und -entlastung) wie Befürchtungen (Entmenschlichung pflegerischen Handelns durch Technikeinsatz) verbunden werden. Während es bislang wenig Daten zur tatsächlichen Anwendung digitaler Technologien im Pflegealltag gibt, belegen empirische Studien, dass Pflegefachkräfte eine große Offenheit bezüglich der Integration von Robotik bis hin zu Künstlicher Intelligenz in das eigene pflegerische Handeln aufweisen: Die von Hofstetter und Kollegen (2019) befragten 324 Pflegekräften signalisierten eine generelle Bereitschaft für den Einsatz solcher Unterstützungssysteme. Interessant ist, dass die Befragten damit insbesondere Unterstützung und Entlastung für patient*innenferne und körperlich anstrengende Tätigkeiten wünschen (ebd.). Auch in einer Erhebung der Berufsgenossenschaft für Gesundheit und Wohlfahrtspflege konnte eine überwiegend positive Einstellung der Pflegenden gegenüber neuen Technologien festgestellt werden. Besonders positiv fiel die Beurteilung der elektronischen Dokumentation aus, etwas weniger die Beurteilung der Robotik (Merda et al. 2017). Auch bei Auszubildenden zeigt sich eine grundsätzliche Offenheit gegenüber neuen Technologien, wie eine Befragung von 415 Auszubildenden in Kranken- und Altenpflegeschulen zeigt. Das eigene Wissen diesbezüglich wird hingegen gering eingeschätzt (Buhtz et al. 2020). Einhellig belegt die aktuelle Studienlage jedoch auch, dass die Pflegefachkräfte die Einrichtungen nur bedingt auf die Digitalisierung vorbereitet einschätzen und sowohl bei den Pflegefachkräften als auch Auszubildenden ein großer Fortund Weiterbildungsbedarf hinsichtlich digitaler Technologien besteht (Buhtz et al. 2020; Kuhlmey et al. 2019; Hofstetter et al. 2019).

Die Bandbreite an Einsatzmöglichkeiten und die Offenheit der Pflegefachkräfte und Auszubildenden korrespondieren mit den hohen Erwartungen an eine Entlastung des Gesundheitssystems (Europäische Kommission 2015) und der Pflegeberufe (Bundesanstalt für Arbeitsschutz und Arbeitsmedizin 2015), die an den Einsatz digitalisierter Technik gestellt werden. Dem steht der aktuelle Stellenwert der Digitalisierung im Kontext der Fachkräftesicherung in der Pflege gegenüber. 


\section{Status quo im Kontext der Fachkräftesicherung}

Vor dem Hintergrund des eingangs konstatierten Fachkräftebedarfs, den es zunehmend schwerer zu decken gelingt, und hinsichtlich der Potenziale von und Erwartungen an die Digitalisierung bezogen auf Arbeitsentlastung und -erleichterung sowie die der damit verbundenen Attraktivitätssteigerung des Berufes werden im nächsten Schritt Ausschnitte eigener empirischer Erhebungen dazu vorgestellt. Das Teilprojekt der Hochschule Esslingen im Forschungsverbund „Zentren für angewandte Forschung an Hochschulen für angewandte Wissenschaften (ZAFH) care4care - Fachkräftebedarf in der Pflege im Zeichen von Alterung, Vielfalt und Zufriedenheit" untersucht anhand einer Delphi-Befragung und mithilfe von Betriebsfallstudien das strategische sowie situative Handeln im Kontext des Fachkräftebedarfs in der Pflege. Im Rahmen der Delphi-Befragung wurden in der Region Neckar-Alb 467 Einrichtungsund Schulleitungen zu ihren implementierten Maßnahmen und wünschenswerten Ideen und den dafür notwendigen Rahmenbindungen befragt. Ziel der Delphi-Befragung war es, Maßnahmen zur Fachkräftegewinnung und -bindung zu identifizieren, die pflegefachliche Einrichtungs- und Schulleitungen als relevant, wirksam und umsetzbar einschätzten. Die Teilnahmequote lag insgesamt bei $26,8 \%$, in den einzelnen Runden zwischen 17,6\% und 14,1\%. Die Auswertung erfolgte deskriptiv; die offenen Antworten wurden thematisch geclustert. In den fokussierten multiperspektivischen Betriebsfallstudien wurden in zwei Einrichtungen der stationären Altenhilfe und in zwei klinischen Einrichtungen in Baden-Württemberg Handlungskonstellationen im Kontext des Fachkräftebedarfs betrachtet und aus der Perspektive unterschiedlicher Akteursgruppen und -ebenen Handlungsideen, konkrete Maßnahmen und wahrgenommene Umsetzungsspielräume untersucht. Dabei wurden Fall-Kontrastierungen der unterschiedlichen Perspektiven innerhalb der Einrichtungen und einrichtungsübergreifend vorgenommen. Insgesamt wurden fünf Interviews mit Führungskräften, vier mit Beschäftigtenvertretungen, vier mit Vertreter*innen von Stabsstellen sowie sieben Gruppendiskussionen mit Pflegefachkräften durchgeführt. Die Daten wurden mittels der Dokumentarischen Methode (Amling \& Vogd 2017) ausgewertet.

Betrachtet man die Ergebnisse beider Studien, lässt sich feststellen, dass das Thema Digitalisierung eine untergeordnete Rolle in der Diskussion der aktuellen Herausforderungen beruflicher Pflege spielt. Im Kontext von Maßnahmen der strategischen Personalentwicklung (z. B. Führungskräfteentwicklung) oder des betrieblichen Gesundheitsmanagements (z. B. belastungssensitives Monitoring von Abteilungen) zeigt sich in der Delphi-Befragung zwar eine hohe Handlungsintensität bzw. ein Handlungswille der befragten Einrichtungs- und Schulleitungen. Im Zusammenhang mit der Fachkräftesicherung wurden An- 
wendungen der Digitalisierung von pflegefachlichen Einrichtungs- und Schulleitungen jedoch selten genannt. Hinsichtlich der Attraktivitätsmerkmale wurde die Antwortoption „digitale, vernetzte, intelligente Technologien“, operationalisiert über die vier Anwendungsbereiche elektronische Pflegedokumentation, technische Assistenzsysteme, Telecare und Robotik, als ein relational zu anderen Merkmalen zu bewertendes Handlungsfeld dargeboten. Unterschieden wurde dabei zwischen „Berufsattraktivität“ als Oberbegriff für die Merkmale, die den Pflegeberuf als solchen attraktiv machen und „Arbeitgeberattraktivität“, dem teilweise andere und weitere Merkmale zugeordnet wurden, auf die die einzelnen Einrichtungen direkt Einfluss nehmen können. Die Leitungs-personen wurden gebeten, die fünf wichtigsten Merkmale der Berufs- und Arbeitgeberattraktivität in eine Rangfolge zu bringen. In beiden Fällen, d. h. sowohl für den Beruf im Allgemeinen, als auch für Arbeitgeber im Besonderen, wurden die digitalen, vernetzten und intelligenten Technologien nach allen anderen Attraktivitätsfaktoren auf den letzten Platz verwiesen. Hierbei zeigten sich keine Unterschiede zwischen den Einschätzungen der Einrichtungs- und Schulleitungen.

Abb. 1: Digitalisierung als Attraktivitätsfaktor? Einschätzung von Leitungspersonen

\begin{tabular}{llll}
\hline & Berufsattraktivität & \multicolumn{2}{c}{ Arbeitgeberattraktivität } \\
\hline Rang-Nr. & Merkmal & Rang-Nr. & Merkmal \\
\hline 1. & Gehalt & 1. & Unternehmenskultur \\
2. & Arbeitsbedingungen & 2. & Arbeitszeit \\
3. & Arbeitszeit & 3. & Führung \\
4. & Berufsprofil & 4. & Arbeitsbedingungen \\
5. & Berufslaufbahn & 5. & Wohnraum \\
6. & Mediale Berichterstattung & 6. & Incentives \\
7. & Lobbyarbeit & 7. & Partizipation \\
8. & Digitale, vernetzte und & 8. & Gestaltung der \\
& intelligente Technologien & & Ausbildung \\
& & 9. & Karrieremöglichkeiten \\
& & 10. & Image \\
& & & Digitale, vernetzte und \\
& & 11. & Technoligente \\
& & & Tegien \\
\hline
\end{tabular}

Anm.: $n=66$; Quelle: eigene Darstellung 
Die Berufsattraktivitätsfaktoren bilden die prominenten Merkmale der öffentlichen Diskussion ab, in der das Gehalt, die Arbeitsbedingungen und die Arbeitszeiten eine herausragende Rolle spielen (bspw. Schmucker 2020; Kamps, Ackermann \& Timmreck 2018). Dass in dieser Rangliste die Digitalisierung eine nachgeordnete Rolle spielt, lässt sich nachvollziehen. Als relationale Attraktivitätsfaktoren, zwischen denen Beschäftigte bei der Wahl ihres Arbeitgebers gewichten, spielen andere Faktoren eine große Rolle wie beispielsweise die Arbeitsatmosphäre und das Führungsverhalten als ,weiche Faktoren“ oder die Arbeitszeiten und -bedingungen. Die Leitungspersonen sehen im Rahmen dieser selbst auf betrieblicher Ebene zu gestaltenden Attraktivitätsmerkmale in der Digitalisierung wenig Potenzial. Auch bei den offenen Antwortformaten in Bezug auf Maßnahmen zur Fachkräftegewinnung und -bindung finden technische Assistenzsysteme, Telecare und Robotik keinerlei Erwähnung. Keine der befragten Einrichtungen verfügt nach eigenen Angaben bislang über eine umfassende Strategie im formalen und inhaltlichen Umgang mit digitalen Technologien. 98,1\% der Einrichtungen stellen zwar Hilfsmittel zur Reduktion der körperlichen Belastung bereit, diese beziehen sich jedoch auf konventionelle Unterstützungssysteme. Der Einsatz neuer Technologien wird lediglich als Idee für die Zukunft formuliert. Auch eine digitale Ausstattung der Berufsschulen ist in vielen Fällen oftmals (noch) nicht vorhanden. In den Berufsschulen kommen bei 58,8\% der befragten Einrichtungen digitale Medien und digitale Lernplattformen zum Einsatz. Hier wird von Berufsbildungspersonen finanzielle Unterstützung für die digitale Ausstattung der Schule als Bedingung für eine adäquate Ausgestaltung der Lernumgebung gefordert.

In den Gruppendiskussionen mit Pflegefachkräften innerhalb der Betriebsfallstudien wurde dem Thema Digitalisierung ebenfalls eine randständige Bedeutung zugewiesen. Sowohl von Pflegefachkräften als auch von Führungskräften wird kaum eine Verbindung zwischen dem Fachkräftemangel und einer möglichen Arbeitserleichterung beispielsweise durch technische Assistenzsysteme, Telecare oder Robotik gezogen. Das Thema „elektronische Pflegedokumentation“ wird von Pflegekräften aufgrund des Umfangs teilweise wenig nutzerfreundlicher Anwendung und/oder ggf. bestehender Defizite der Anwendungskompetenz eher als zusätzliche Arbeitsbelastung beschrieben.

Jetzt geht da vieles über den PC. Aber das Problem war halt, dass viele mit dem PC gar nicht umgehen konnten. Da mussten wir erst einmal die PCGrundkenntnisse wissen und lernen. Wie funktioniert das alles? Also ich habe heute immer noch Schwierigkeiten, ja? Wo was ist, wo kann ich was finden. (Fachkraft, Betrieb C)

In einer anderen Einrichtung formuliert eine Fachkraft den Wunsch nach einer besser handhabbaren Softwarelösung, von der sie sich Zeitersparnis durch den Abbau bürokratischer Arbeitsschritte erhofft. Als Folge eines solchen in den 
Arbeitsalltag gut integrierten digitalen Assistenzsystems wäre nach Ansicht der Pflegeperson durch den damit verbundenen Zeitgewinn eine Steigerung der Pflegequalität erwartbar.

Wenn Kliniken Geld in die Hand nehmen würden, um ein vernünftiges Computersystem zu installieren. Man würde eine Menge Arbeitszeit sparen, ja? [...] Also alleine unser System [...] wie oft klicke ich da etwas an und WARTE dann erstmal einfach 30 Sekunden, bis das blöde Ding dann geladen ist. Also da geht so viel Zeit flöten. Wenn das viel kompakter und einfach und übersichtlicher gemacht wird, dann hätten ich nachher auch wiederum mehr Zeit am Patienten. Und nicht mit der ganzen Bürokratie. (Fachkraft, Betrieb D)

Die beiden beispielhaft ausgewählten Zitate verdeutlichen, dass das Thema Digitalisierung im Kontext der steigenden Dokumentationsanforderungen behandelt und mitunter als Synonym für die zunehmende Bürokratisierung pflegerischen Handelns verwendet wird. Dieser Betrachtung digitaler Anwendungen als ,notwendiges Übel', welches Mehrbelastung und Unsicherheiten mit sich bringt, steht auf der anderen Seite die Hoffnung auf eine spürbare Arbeitserleichterung durch praktikable und praxisnahe Systeme gegenüber.

Zusammenfassend lässt sich festhalten, dass Digitalisierung vorwiegend im Kontext von Planung, Dokumentation und Ausbildung diskutiert wird. Im Hinblick auf Unterstützungspotenziale der Mitarbeiter*innen bspw. im Sinne der betrieblichen Gesundheitsförderung spielen neue Technologien im pflegerischen Berufsalltag noch keine Rolle. Auch im Hinblick auf die Berufsund Arbeitgeberattraktivität wird der Digitalisierung kaum Potenzial beigemessen.

Die im Zuge der Umsetzung des Pflegeberufegesetzes anstehende Ausbildungsreform könnte nun auch dazu genutzt werden, das Potenzial der Digitalisierung in einer dem Primat des pflegerischen Handelns unterstützenden Funktion zu sehen und die dafür erforderlichen Kompetenzen so anzulegen, dass sie integriert sind in konstitutive pflegerische Kompetenzen. Welche Anknüpfungspunkte sich dafür in den ab 2020 geltenden rechtlichen Grundlagen und den Rahmenlehr- und Rahmenausbildungsplänen bieten, wird im nächsten Schritt dargelegt. 


\section{Die Rolle der Digitalisierung in der Pflegeausbildung ab 2020}

Der zuvor vorgestellte kursorische Einblick in Forschungsergebnisse, die Auskunft geben über den Status quo von digitalen (Nicht-)Anwendungen im Kontext von Fachkräftesicherung legt die Frage nahe, ob die nun anstehende Ausbildungsreform in der Pflege zugleich auch ein Motor sein kann für eine intensivere Nutzung dieses Potenzials. Über die Ausbildung der kommenden Generation beruflich Pflegender könnten sinnvolle Anwendungen von Digitalisierung zur Arbeitsentlastung sowie zur Attraktivitätssteigerung stärker in die Versorgungsrealität integriert werden. Der Stellenwert des Themas Digitalisierung wird deshalb im nächsten Argumentationsschritt anhand des Pflegeberufegesetzes mit seiner Ausbildung- und Prüfungsverordnung sowie der diese gesetzlich-formalen Grundlagen konzeptionell-inhaltlichen Operationalisierung, die zwischenzeitlich in Form der Rahmenlehr- und Rahmenausbildungspläne vorliegt, diskutiert.

Im Pflegeberufegesetz (BGB1. [2017] I, 2581-2614) selbst findet sich das Thema Digitalisierung in expliziter Weise lediglich im Kontext der nun neu hinzugekommenen hochschulischen Ausbildung. Hier wird als Zielbeschreibung die Kompetenz benannt, neue Technologien in das berufliche Handeln $\mathrm{zu}$ integrieren und dahingehende Fort- und Weiterbildungsbedarfe erkennen zu können ( $\$ 37$ Abs. 3).

In der Ausbildungs- und Prüfungsverordnung (BGBl. [2018] I, 15721621) taucht das Thema Digitalisierung an wenigen Stellen in den Anlagen zu den geforderten Kompetenzen für Zwischen- und Abschlussprüfung auf. Pflegemaßnahmen und Beobachtungen sollen ,auch unter Zuhilfenahme digitaler Dokumentationssysteme“ (ebd., 1592) dokumentiert werden. Neben analogen sollen auch digitale Pflegedokumentationssysteme genutzt werden, um „Pflegeprozessentscheidungen [...] selbständig und im Pflegeteam zu evaluieren (ebd., 1596, 1601). Im Hinblick auf technische Assistenzsysteme werden in Anlage 2 unter dem Kompetenzbereich I „Pflegeprozesse und Pflegediagnostik in akuten und dauerhaften Pflegesituationen verantwortlich planen, organisieren, gestalten, durchführen, steuern und evaluieren“" und der Subkategorie „Entwicklung und Autonomie in der Lebensspanne fördern“ als Ziel formuliert, dass beruflich Pflegende durch rehabilitative Maßnahmen zum Erhalt und zur Wiedererlangung von Alltagskompetenz bei[tragen] und hierzu auch technische Assistenzsysteme in das pflegerische Handeln [integrieren]" (ebd., 1593) sowie Potenziale und Grenzen reflektieren. Die Reflexion und Begründung des eigenen Handelns (Kompetenzbereich IV) bezieht sich auch darauf, „den Einfluss [...] technologischer Entwicklungen auf die Versorgungsverträge und Versorgungsstrukturen im Gesundheits- und Sozialsystem [zu erfassen]“ (BGBl. [2018] I, 1604). Die Nutzung „moderner 
Informations- und Kommunikationstechnologien“ als „Element der persönlichen und beruflichen Entwicklung" (ebd., 1605) wird in Kompetenzbereich V explizit im Hinblick auf das lebenslange Lernen erwähnt.

Die durch eine vom Bundesgesundheitsministerium und vom Bundesministerium für Familie, Senioren, Frauen und Jugend einberufene und eingesetzte Fachkommission (gemäß $\S 53$ PflBG) erarbeiteten Rahmenlehrpläne und Rahmenausbildungspläne bilden zukünftig die konzeptionellinhaltliche Grundlage für die curriculare Ausgestaltung der Pflegeausbildung (Fachkommission 2019). Im Zusammenhang mit der Lernkompetenz wird hier zu Ausbildungsbeginn auf die Reflexion der eigenen digitalen Kompetenzen verwiesen (ebd., 38). Insgesamt findet hier das Thema der Digitalisierung in fast allen curricularen Einheiten der Rahmenlehrpläne und Rahmenausbildungspläne Berücksichtigung. Dies wird nachfolgend anhand von Beispielen entlang der in Kapitel 2 eingeführten Systematik illustriert.

Software für die Planung, Steuerung und Dokumentation pflegerischen Handelns: In den Rahmenlehrplänen wird im Kontext des Pflegeprozesses in naheliegender Weise innerhalb der curricularen Einheiten auf die Nutzung von elektronischer Pflegedokumentation verwiesen. Als Handlungsmuster, d. h. für eine situationsspezifische Aufbereitung der Kompetenzen, wird angegeben, dass Auszubildende ,,mit Pflegedokumentationssystemen (analog/ digital) umgehen“, „durchgeführte Pflege dokumentieren“ und den Pflegedokumentationssystemen „Informationen entnehmen“ können (bspw. ebd., 48). Die Hinweise in den Rahmenausbildungsplänen korrespondieren mit den Inhalten der Rahmenlehrpläne (bspw. ebd., 310).

Technische Assistenzsysteme: Im Hinblick auf technische Assistenzsysteme finden sich in den Rahmenlehrplänen mehrere Anwendungsszenarien. Neben dem Einsatz wird hier insbesondere die Information und Schulung bzgl. dieser Technologien betont, bspw. im Kontext rehabilitativen Pflegehandelns (bspw. ebd., 133, 135). Innerhalb der curricularen Einheit „CE 08 Menschen in kritischen Lebenssituationen und in der letzten Lebensphase begleiten" wird als Handlungsmuster für das letzte Ausbildungsdrittel die „Verwendung digitaler Begleiter/Smart Home Technik“ (ebd., 163). erwähnt. In den Rahmenausbildungsplänen wird die Recherche, Beratung (bspw. ebd., 261) und Anleitung im Umgang mit digitalen Assistenzsystemen (bspw. ebd., 307) aufgeführt.

Telecare/-nursing: Im Rahmenlehrplan werden an einer Stelle „Technische/digitale Hilfsmittel für gesundheitsförderliche/präventive Informations- und Beratungsangebote [...] (z. B. Telecare etc.)“ und deren kritische fachliche Reflexion erwähnt (Fachkommission 2019, 63). Verortet ist diese Kompetenz als Handlungsmuster innerhalb der curricularen Einheit „CE 04 Gesundheit fördern und präventiv handeln“. In den Rahmenausbildungsplänen wird Telecare nicht weiter erwähnt. 
Robotik: Schließlich könnten in den Rahmenlehrplänen Anwendungen aus dem Bereich der Robotik z. B. im Kontext von Mobilität innerhalb der curricularen Einheit „CE $02 \mathrm{Zu}$ pflegende Menschen in der Bewegung und Selbstversorgung unterstützen“ verborgen sein. Unter Handlungsmuster wird als Kompetenz beschrieben, dass die Auszubildenden ,Menschen bei Alltagsaktivitäten in ihrer Mobilität unterstützen und bei Bedarf technische und digitale Hilfsmittel nutzen“ (a. a. O., 45). Diese werden nicht weiter konkretisiert. In den Rahmenausbildungsplänen wird der Einsatz geeigneter technischer ,,(auch digital unterstützter) Hilfsmittel“" bspw. im Zusammenhang mit der Förderung der Autonomie erwähnt (a. a. O., 261).

Zusammenfassend lässt sich konstatieren, dass sich Digitalisierung in ihrer Bandbreite wenig innerhalb der neuen rechtlichen Grundlagen im Unterschied dazu jedoch in den Rahmenlehr- und Rahmenausbildungsplänen in divergierendem Maße wiederfindet. An unterschiedlichen Stellen werden Formen digitaler Unterstützung im Kontext des Pflegeprozesses genannt. Insgesamt bleibt die Abbildung neuer Technologien jedoch oft punktuell und stellenweise optional.

\section{Fazit und Ausblick}

Die geringe Bedeutung von Digitalisierung im aktuellen pflegerischen Alltag und im Kontext der Fachkräftesicherung korrespondiert mit Ergebnissen weiterer Studien, die belegen, dass elaboriertere Formen und Anwendungen der Digitalisierung bislang kaum eine Rolle spielen (bspw. Zöllick et al. 2020; Boll-Westermann et al. 2019; Merda et al. 2017). Dies kann u. a. darin begründet sein, dass bisherige Forschungsaktivitäten durch einen starken Technikfokus und mit geringer Adaption in Bezug auf die Spezifika und Belange pflegerischen Handelns charakterisiert waren (Daum 2017). Vor dem Hintergrund neuerer Befunde zur Technikakzeptanz von Pflegekräften, die sich durchaus Entlastung und Unterstützung durch pflegeangepasste digitale Anwendungen vorstellen und wünschen (Hofstetter et al. 2019; Merda et al. 2017), ist ein Desiderat in Bezug auf sowohl Realisation als auch grundlagen- und anwendungsbezogener Forschung zu konstatieren. Aber auch in der berufsschulischen Ausbildung finden digitale Technologien wenig Anwendung. Dies ist jedoch Grundvoraussetzung des Erwerbs digitaler Kompetenzen. Diese Lücke wurde erkannt: Um die Durchdringungsrate zu erhöhen, werden zukünftig Pflegeschulen in den „Digitalpakt Schule“ einbezogen und somit auch finanziell bei der digitalen Ausstattung unterstützt (Bundesministerium für Familie, Senioren, Frauen und Jugend 2019).

Der geringe Stellenwert digitaler Technologien im Pflegeberufegesetz könnte auf den langandauernden Diskussions- und Verabschiedungsprozess 
des Gesetzes zurückzuführen sein. Der Entstehungsprozess des Pflegeberufegesetzes lässt sich auf ein Eckpunktepapier im Jahr 2012 zurückführen (Kriesten 2016). Da es um eine grundlegende Neuausrichtung der Pflegeausbildung ging, könnten im Rahmen grundsätzlicher Diskussionen digitale Technologien auch aufgrund des derzeit noch geringen Stellenwerts in der Praxis an dieser Stelle nicht umfänglich berücksichtigt worden sein. Die solitäre Erwähnung im Pflegeberufegesetz in Bezug zur hochschulischen Ausbildung kann darin begründet liegen, dass an dieser Stelle wissenschaftsorientierte Kompetenzen im Sinne evidenzbasierter Pflege zu Grunde gelegt werden, die eine hochschulische Ausbildung voraussetzen.

In der neuen Pflegeausbildung lässt sich eine Konkretisierung der Anwendungsformen und -bezüge digitaler Technologien vom Pflegeberufegesetz bis zu den Rahmenlehr- und Rahmenausbildungsplänen erkennen. Die für eine effektiv-entlastende und zugleich kritisch-konstruktive Nutzung digitaler Anwendungen in der Pflegepraxis erforderlichen Kompetenzen finden insgesamt jedoch nur bedingt Berücksichtigung. Für eine wirksame und sinnvolle Ergänzung und Unterstützung pflegerischen Handelns mit hohen Entlastungspotenzialen hinsichtlich der Belastungsstärke (insb. bei körperlich stark strapazierenden Tätigkeiten) und Belastungsdichte (z. B. maximale Vereinfachung von Routinetätigkeiten und Standardabläufen) müsste sich Technikkompetenz jedoch konsequenter als Querschnittskompetenz abbilden, damit die Potenziale der Digitalisierung im Rahmen aller pflegerischen Kernprozesse bereits mitgedacht werden. Neben Kernkompetenzen in Pflegeinformatik (Hübner et al. 2017) sind bspw. Kompetenzen zu ethischen Fragestellungen des Einsatzes neuer Technologien (Manzeschke et al. 2013) oder auch Beratungskompetenzen (Büscher, Oetting-Roß \& Sulmann 2016) für die Auseinandersetzung mit der digitalen Transformation erforderlich. Für den Erwerb digitaler Kompetenzen sollte als Grundlage der curricularen Implementierung ein ,multiprofessionell ausgerichtetes Mustercurriculum [...] unter Beteiligung aller relevanten Stakeholder erstellt werden“" (Kuhn et al. 2019).

Im Zuge einer weiteren Profilierung des Berufsbildes wäre es erforderlich, Digitalisierung als integralen Bestandteil zu fassen: Nicht lediglich als weiteres (möglicherweise lästiges) inhaltliches Add-on in einem ohnehin umfangreichen Inhaltskatalog, sondern als Querschnittskompetenz zur pflegerischen Kern- und Kontextkompetenz unter der Voraussetzung, dass die beruflich Pflegenden den Einsatz digitalisierter Technik definieren und steuern. Voraussetzung dafür wären empirisch abgesicherte Konzepte einer weitreichenden Arbeitsteilung, die die Potenziale von digitaler Technik größtmöglich zur Entlastung so nutzt, dass die doppelte Handlungslogik pflegerischen Handelns als analytischer Problemlösungsprozess und zugleich als hermeneutisches Fallverstehen den Ausgangs- und Bezugspunkt von Technikeinsatz und Digitalisierung bilden. In diesem Sinne wäre jede 
Erprobung und Umsetzung der Technikunterstützung in der Pflege als „,soziotechnische Innovation“ (Fuchs-Frohnhofen et al. 2018, 10f.) anzulegen, nämlich passgenau zu pflegerischen Arbeits- und Organisationsprozessen unter dem Primat der Spezifika professionellen Pflegehandelns und der spezifischen Handlungslogik professionellen Pflegehandelns als Interaktionsarbeit mit doppelter Handlungslogik (Fuchs-Frohnhofen et al. 2018; HülskenGiesler \& Daxberger 2018). Dies erfordert in Bezug auf das Berufsprofil eine auf das jeweilige Qualifikationsniveau abgestimmte Definition von Verantwortungsbereichen innerhalb des Einsatzes digitaler Technologien.

Für eine breite Akzeptanz in der Praxis wären zunächst eine begriffliche Klärung und Systematisierung erforderlich, um die Potenziale und Risiken neuer Technologien professionsimmanent kommunizier- und verhandelbar $\mathrm{zu}$ machen (PricewaterhouseCoopers 2016). Entgegen der bisherigen eher technikgetriebenen Forschung und Entwicklung (bspw. Kuhn et al. 2019; Evans et al. 2018) könnten digital unterstützte Lösungen stärker von der Professionslogik her passgenau entwickelt und implementiert werden, so dass wirksame Effekte von sinnvoller Arbeitsentlastung eintreten, die zur Attraktivität des Berufs insgesamt beitragen. Eine Weiterentwicklung des Berufsprofils dahingehend, dass technisch-digitale Anwendungen in pflegefachlich dominierter Perspektive integriert werden, könnte somit einen spürbaren Beitrag für die Fachkräftesicherung leisten. Im Kontext dieser Weiterentwicklung des Berufsprofils spielt die Ausbildung eine zentrale Rolle. Insofern stellt sich nun für die curriculare Umsetzung der Vorgaben die Herausforderung, pflegeadaptive Anwendungen der Digitalisierung konstitutiv zu verankern.

\section{Literatur}

Amling, S. \& Vogd, W. (2017). Dokumentarische Organisationsforschung. Perspektiven der praxeologischen Wissenssoziologie. Opladen, Berlin, Toronto: Verlag Barbara Budrich.

Ammende, R. et al. (2019). Rahmenlehrpläne der Fachkommission nach § 53 PflBG: Rahmenlehrpläne für den theoretischen und praktischen Unterricht. Rahmenausbildungspläne für die praktische Ausbildung. Online: https://www.bibb.de/dokumente/pdf/geschst_pflgb_rahmenplaene-der-fachkommission.pdf (04.04.2020).

Böhle, F. \& Glaser, J. (2006). Interaktion als Arbeit - Ausgangspunkt. In F. Böhle \& J. Glaser (Hrsg.), Arbeit in der Interaktion. Interaktion als Arbeit (1. Aufl., S. 1115). Wiesbaden: VS Verlag für Sozialwissenschaften. 
Boll-Westermann, S., Hein, A., Heuten, W. \& Krahn, T. (2019). Pflege 2050 - Wie die technologische Zukunft der Pflege aussehen könnte. In Zentrum für Qualität in der Pflege (Hrsg.), Pflege und digitale Technik (S. 10-15). Online: https://www.zqp.de/wp-content/uploads/ZQP-Report-Technik-Pflege.pdf (04.04.2020).

Büscher, A., Oetting-Roß, C. \& Sulmann, D. (2016). Qualitätsrahmen für Beratung in der Pflege. Hrsg. v. Zentrum für Qualität in der Pflege. Online: https://www.zqp.de/wp-content/uploads/Qualitaetsrahmen_Beratung_Pflege.pdf (25.03.2020).

Buhtz, C., Paulicke, D., Hofstetter, S. \& Jahn, P. (2020). Technikaffinität und Fortbildungsinteresse von Auszubildenden der Pflegefachberufe. Eine Onlinebefragung. HBScience, 260, 162-173.

Buhtz, C. et al. (2018). Technische Assistenzsysteme in der Pflegeausbildung. Eine Online-Befragung. Online: https://format.medizin.uni-halle.de/wp-content/uploads $/ 2019 / 08 /$ Brosch\%C3\%BCre_Sch\%C3\%BClerbefragung_Web-Version.pdf (10.12.2019).

Bundesagentur für Arbeit (2018). Fachkräfteengpassanalyse Juni 2018. Online: http://m.vpt.de/fileadmin/user_upload/news/pdf/BA-FK-Engpassanalyse-2018-06 .pdf (13.09.2019).

Bundesanstalt für Arbeitsschutz und Arbeitsmedizin (2015). Intelligente Technik in der beruflichen Pflege. Von den Chancen und Risiken einer Pflege 4.0. Dortmund: Bundesanstalt für Arbeitsschutz und Arbeitsmedizin.

Bundesministerium für Familie, Senioren, Frauen und Jugend (2019). Ausbildungsoffensive Pflege (2019-2023). Vereinbarungstext. Ergebnis der Konzertierten Aktion Pflege / $A G$ 1. Online: https://www.bmfsfj.de/blob/jump/135564/ausbildungsoffensive-pflege-2019-2023-data.pdf (25.03.2020).

Bundesrepublik Deutschland (2018). Ausbildungs- und Prüfungsverordnung für die Pflegeberufe (Pflegeberufe-Ausbildungs- und Prüfungsverordnung PflAPrV). Vom 02. Oktober 2018. Bundesgesetzblatt Teil I, 34, 1572-1621.

Bundesrepublik Deutschland (2017). Gesetz zur Reform der Pflegeberufe (Pflegeberufegesetz - PflBG). Vom 17. Juli 2017. Bundesgesetzblatt Teil I, 49, 2581-2614.

Daum, M. (2017). Digitalisierung und Technisierung der Pflege in Deutschland. Aktuelle Trends und ihre Folgewirkungen auf Arbeitsorganisation, Beschäftigung und Qualifizierung. Unter Mitarbeit von U. Ploch und T. Werkmeister. Hrsg. v. DAAStiftung Bildung und Beruf. INPUT Consulting GmbH. Online: http://www.daastiftung.de/fileadmin/user_upload/ digitalisierung_und_technisierung_der_pflege 2.pdf (25.03.2020).

Europäische Kommission (2015). Innovation for active and healthy ageing. European summit on innovation for active and healthy ageing. Final report. Online: http://ec.europa.eu/research/innovation-union/pdf/active-healthy-ageing/ageing_ summit_report.pdf (25.03.2020).

Evans, M., Hielscher, V. \& Voss, D. (2018). Damit Arbeit 4.0 in der Pflege ankommt. Wie Technik die Pflege stärken kann. Hrsg. v. Hans-Böckler-Stiftung (Policy Brief, 004). Online: https://www.boeckler.de/pdf/p_fofoe_pb_004_2018.pdf (25.03.2020).

Fehst, P. (2016). Bewältigungsstrategien für den Fachkräftemangel in der Pflege. Können wir ausreichend Nachwuchs im Pflegeberuf rekrutieren und halten? München: Studylab. 
Flake, R., Kochskämper, S., Risius, P. \& Seyda, S. (2018). Fachkräfteengpass in der Altenpflege. Hrsg. v. Institut der deutschen Wirtschaft (IW-Trends, 3). Online: https://www.econstor.eu/handle/10419/194600 (04.04.2020).

Fuchs-Frohnhofen, P. et al. (2018). Memorandum "Arbeit und Technik 4.0 in der professionellen Pflege". Online: http://www.memorandum-pflegearbeit-und-technik.de/files/memorandum/layout/js/Memorandum_AuT_Pflege_4_0.pdf (04.04.2020).

Gerhardt, A. L. \& Kanzog, J. (2017). Ausbildungsreport 2017. Online: http://jugend.dgb.de/++co++17e7ad30-8226-11e7-83fc-525400d8729f/Ausbildungsrepor t-2017.pdf (25.03.2020).

Hofstetter, S., Richey, V. \& Jahn, P. (2019). Digitale Revolution. Survey zur Akzeptanz sozial assistiver Technologie in der Pflege. Die Schwester, Der Pfleger, 58(9), 3234.

Hübner, U., Egbert, N., Hackl, W., Lysser, M., Schulte, G., Thye, J. \& Ammenwerth, E. (2017). Welche Kernkompetenzen in Pflegeinformatik benötigen Angehörige von Pflegeberufen in den D-A-CH-Ländern? Eine Empfehlung der GMDS, der ÖGPI und der IGPI. Online: https://www.egms.de/static/pdf/journals/mibe/201713/mibe000169.pdf (25.03.2020).

Hübner, U., Liebe, J.-D., Hüsers, J., Thye, J., Egbert, N., Hackl, W. \& Ammenwerth, E. (2015). Schwerpunkt Pflege im Informationszeitalter. Hrsg. v. Hochschule Osnabrück (IT-Report Gesundheitswesen). Online: https://www.hs-osnabrueck.de/fileadmin/HSOS/Homepages/Forschungsgruppe_Infomatik_im Gesund heitswesen/Pflege_im_Informationszeitalter_2015.pdf (25.03.2020).

Hülsken-Giesler, M. \& Daxberger, S. (2018). Robotik in der Pflege aus pflegewissenschaftlicher Perspektive. In O. Bendel (Hrsg.), Pflegeroboter (S. 125-139). Wiesbaden: Springer Gabler.

Isfort, M. \& Gessenich, H. (2019). Analysen zur Pflegeentwicklung ausgewählter Regionen in Baden-Württemberg. Hochschule Esslingen. Online: https://www.zafhcare4care.de/fileadmin/user_upload/DIP_Bericht_BW_Regional.pdf (25.03.2020).

Kamps, E., Ackermann, D. \& Timmreck, C. (2018). Pflegestudie 2018. Wege aus dem Fachkräftemangel in der stationären Altenpflege. Online: https://www.hs-niederrhein.de/fileadmin/dateien/FB10/PFLEGESTUDIE.pdf (25.03.2020).

Koehler, F. (2018). Efficacy of telemedical interventional management in patients with heart failure (TIM-HF2). A randomised, controlled, parallel-group, unmasked trial. The Lancet, 392, 1047-1057.

Kriesten, U. (2016). Altenpflegeausbildung in Deutschland. Diskussion der Prognosen zu einer Integration der Altenpflegeausbildung in eine generalistische Pflegeausbildung. Online: https://d-nb.info/1122111045/34 (25.03.2020).

Kuhlmey, A., Blüher, S., Nordheim, J. \& Zöllick, J. C. (2019). Technik in der Pflege Einstellungen von professionell Pflegenden zu Chancen und Risiken neuer Technologien und technischer Assistenzsysteme. Projektbericht für das ZQP. Online: https://www.zqp.de/wp-content/uploads/ZQP-Bericht-Technik-profPflege.pdf (04.04.2020).

Kuhn, S. et al. (2019). Wie revolutioniert die digitale Transformation die Bildung der Berufe im Gesundheitswesen? (careum working paper, 8). Online: www.careum.ch/workingpaper8-lang (04.04.2020). 
Lauterbach, M. \& Hörner, K. (2019). Erfolgsfaktoren in der Digitalisierung der Gesundheitsversorgung. In R. Haring (Hrsg.), Gesundheit digital. Perspektiven zur Digitalisierung im Gesundheitswesen (S. 123-142). Berlin, Heidelberg: Springer.

Manzeschke, A., Weber, K., Rother, E. \& Fangerau, H. (2013). Ethische Fragen im Bereich Altersgerechter Assistenzsysteme. Ergebnisse der Studie. Online: https://www.technik-zum-menschen-bringen.de/dateien/service/broschuere-ethische-fragen-altersgerechte-assistenzsysteme.pdf/download (25.03.2020).

Merda, M., Schmidt, K. \& Kähler, B. (2017). Pflege 4.0-Einsatz moderner Technologien aus der Sicht professionell Pflegender. Online: https://www.bgw-online.de/DE/Medien-Service/Medien-Center/Medientypen/BGW-Broschueren/BG W09-14-002-Pflege-4-0-Einsatz-moderner-Technologien.html (25.03.2020).

Pöser, S. \& Bleses, P. (2018). Digitalisierung der Arbeit in der ambulanten Pflege im Land Bremen: Praxis und Gestaltungsbedarfe digitaler Tourenbegleiter (Arbeit und Wirtschaft in Bremen, 25). Online: https://www.econstor.eu/bitstream/10419/179518/1/102418854X.pdf(25.03.2020).

PricewaterhouseCoopers (2016). Weiterentwicklung der eHealth-Strategie. Studie im Auftrag des Bundesministeriums für Gesundheit. Berlin: PricewaterhouseCoopers (PwC).

Robert-Koch-Institut (2015). Gesundheit in Deutschland. Online: www.gbebund.de/gbe10/owards.prc_show_pdf?p_id=22302\&p_sprache $=\mathrm{d} \& p \_u i d=$ gast $\& p$ aid=85131451\&p_lfd_nr=2 (04.04.2020).

Rösler, U. et al. (2018). Digitalisierung in der Pflege. Wie intelligente Technologien die Arbeit professionell Pflegender ändern. Berlin: Geschäftsstelle der Initiative Neue Qualität der Arbeit. Bundesanstalt für Arbeitsschutz und Arbeitsmedizin.

Schmucker, R. (2020). Arbeitsbedingungen in Pflegeberufen. In K. Jacobs, A. Kuhlmey \& S. Greß (Hrsg.). Pflege-Report 2019. Mehr Personal in der Langzeitpflege aber woher? (S. 49-60). Berlin: Springer Open.

Vereinigung der Bayerischen Wirtschaft e. V (2017). Digitalisierung als Rahmenbedingung für Wachstum. Online: https://www.vbw-bayern.de/Redaktion/Frei-zugaengliche-Medien/Abteilungen-GS/Volkswirtschaft/2017/Download s/Studie-Digitalisierung-als-Rahmenbedingung-f\%C3\%BCr-Wachstum_2017.pdf (25.03.2020).

Zöllick, J. C., Kuhlmey, A., Suhr, R., Eggert, S., Nordheim, J. \& Blüher, S. (2020). Akzeptanz von Technikeinsatz in der Pflege. Zwischenergebnisse einer Befragung unter professionell Pflegenden. In K. Jacobs, A. Kuhlmey \& S. Greß (Hrsg.). Pflege-Report 2019. Mehr Personal in der Langzeitpflege - aber woher? (S. 211218). Berlin: Springer Open. 


\title{
Erwartungen an Praxissemester im Studium für ein Lehramt an beruflichen Schulen - eine qualitative Studie im Rahmen der Studiengangentwicklung
}

\author{
Andrea Burda-Zoyke und Immo Degner
}

\section{Einführung}

Seit einigen Jahren ist in den Studiengängen des beruflichen Lehramts ebenso wie in allgemeinbildenden Lehramtsstudiengängen ein deutlicher Trend zur Ausweitung der schulpraktischen Anteile zu verzeichnen, welcher sich insbesondere in der Einführung von Praxissemestern ${ }^{1}$ zeigt (Frommberger \& Lange 2018; Schüssler \& Weyland 2014; Tramm \& Naeve-Stoß 2018; Weyland \& Wittmann 2015, 2017; Weyland, Gröschner \& Kosinár 2019). Allerdings existieren bundesweit zahlreiche standortspezifische Konzeptionsvarianten der sogenannten Praxissemester (Gröschner \& Hascher 2019), auch in lehrerbildenden Studiengängen für berufliche Schulen (Weyland et al. 2019). Im Folgenden wird für eine erste Annäherung unter einem Praxissemester ein spezifisches Format von Langzeitpraktika verstanden, welches neben der Teilnahme an einer schulpraktischen Phase idealtypisch auch dessen hochschulische Vorund Nachbearbeitung sowie Begleitung umfasst (Frommberger \& Lange 2018; Weyland 2016; Weyland et al. 2019). Zudem wird das Praxissemester - in Abgrenzung zum Vorbereitungsdienst - als Studienelement verstanden, welches vorrangig dem Ziel der Professionalisierung der angehenden Lehrkräfte dient (Lange \& Frommberger 2017; Pätzold 2018; Seifried \& Wuttke 2017; Tramm \& Naeve-Stoß 2018; Weyland \& Wittmann 2011, 2015, 2017; Weyland et al. 2019). Allerdings zeigt sich in ersten Studien, dass die am Praxissemester beteiligten Akteur*innen trotz des gemeinsam geteilten übergeordneten Ziels der Professionalisierung im Detail durchaus divergente Teilziele verfolgen (Großmann, Bach \& Winkel 2017; Naeve-Stoß \& Tramm 2017; Weyland \& Wittmann 2011).

Die erforderliche curriculare Integration von Praxissemestern (Frommberger \& Lange 2018; Schubarth 2017; Tramm \& Naeve-Stoß 201; Weyland et al. 2019) legt wiederum eine Studiengangentwicklung ${ }^{2}$ nahe, in deren Rahmen

1 Zur Problematisierung des Begriffs „Praxissemester“ unter Berücksichtigung der bundesweiten sprachlichen Ausprägungsformen siehe u. a. Weyland et al. $(2019,11)$.

2 Unter Studiengangentwicklung wird in diesem Beitrag aus Gründen der besseren Lesbarkeit neben der Entwicklung von neuen Studiengängen auch die weitaus häufigere Variante der Weiterentwicklung von bereits existierenden Studiengängen gefasst. 
die unterschiedlichen Ziele der Akteur*innen eingebracht und berücksichtigt werden sollten. Auf die für den Erfolg der Praxissemester notwendige Zielklärung zwischen den im Rahmen der Durchführung beteiligten Akteuren wurde in verschiedenen Studien bereits hingewiesen (Weyland \& Wittmann 2017; König \& Rothland 2018). Jedoch ist bisher weitgehend unbeleuchtet, mit welchen Erwartungen die an der Studiengangentwicklung beteiligten Akteur*innen in diesen Entwicklungsprozess eintreten und inwiefern auch in dieser Phase Gemeinsamkeiten und Unterschiede vorliegen, welche es zu berücksichtigen gilt.

Daher geht dieser Beitrag der Frage nach, welche Erwartungen die an der Studiengangentwicklung beteiligten wesentlichen Akteur*innen mit der Einführung eines Praxissemesters in das Studium für ein Lehramt an beruflichen Schulen verbinden. Hierzu wird zunächst ein kurzer Überblick über den Forschungsstand in Hinblick auf die Erwartungen zum Praxissemester gegeben (Kap. 2). Dieser bildet die Basis für die Auswertung des empirischen Datenmaterials aus einer qualitativen interviewbasierten Studie mit Akteuren der Studiengangentwicklung. Nach der Skizzierung des Forschungsdesigns (Kap. 3) werden die wesentlichen Ergebnisse dargestellt und kurz diskutiert (Kap. 4). Eine kurze Zusammenfassung der Ergebnisse sowie ein Ausblick auf weitere Forschungsdesiderate schließen den Beitrag ab (Kap. 5). Die referierten Ergebnisse sollen dazu beitragen, mögliche Gemeinsamkeiten und Diskrepanzen in den Erwartungen zwischen den an der Studiengangentwicklung bzw. der Entwicklung des intendierten Curriculums beteiligten Akteursgruppen aufzudecken. Diese können auch anderen Standorten im Rahmen der Studiengangentwicklung Ansatz- bzw. Diskussionspunkte für die Klärung von bzw. für die Verständigung über die Erwartungen bezüglich des Praxissemesters liefern, welche letztlich die Konzeption und Umsetzung auch der universitären Begleitveranstaltungen prägen können. Dies kann dann im Idealfall zu einer kohärenten Gestaltung der Begleitseminare unter Berücksichtigung der curricular verankerten Ziele der Praxissemester beitragen, der eine besondere Bedeutung beigemessen wird (Weyland \& Wittmann 2017 in Anlehnung an Gröschner, Schmitt \& Seidel 2013).

\section{Forschungsstand und theoretische Basis}

Mit dem Praxissemester als Studienelement rückt insbesondere das Ziel der Professionalisierung der angehenden Lehrkräfte in den Blick. Unter Berücksichtigung wesentlicher bildungspolitischer Programme, Ordnungsgrundlagen und empirischer Studien kann die Professionalisierung zusammenfassend über folgende Teilziele ausdifferenziert werden (Weyland \& Wittmann 2015, 2017; Weyland 2016, 2019; Weyland et al. 2019; Tramm \& Naeve-Stoß 2018; König 
\& Rothland 2018; Mertens, Schlag \& Gräsel 2018; Seifert \& Schaper 2018; Brenneke, Pfaff, Schrader \& Tervooren 2018):

- Förderung unterrichts- bzw. handlungspraktischer Fähigkeiten

- Förderung (methodengeleiteter) theoretischer Reflexionsfähigkeit

- Relationierung von Theorie und Praxis und die Relevanz von wissenschaftlichem Wissen erkennen

- Anbahnung einer forschenden Grundhaltung und Entwicklung von Forschungskompetenz

- Reflexion der eigenen Kompetenzentwicklung und Berufswahlüberprüfung

Allerdings zeigt sich in Befragungen, dass die Zielsetzungen zwischen den Akteursgruppen im Praxissemester (z. B. Studierende, Lehrkräfte an Hochschulen und in Schulen) variieren können (Großmann et al. 2017; Naeve-Stoß \& Tramm 2017). Während bei den Studierenden tendenziell eine Präferenz für unterrichtspraktische Fähigkeiten festzustellen ist, weisen Hochschullehrende eher die (methodengeleitete) theoretische Reflexionsfähigkeit, die Relationierung von Theorie und Forschung sowie die Anbahnung einer Forschenden Grundhaltung aus.

Andererseits zeichnen sich auch Herausforderungen und Problemlagen ab, welche die Akteure mit einem Praxissemester verbinden, und die zu folgenden Schwerpunkten zusammengefasst werden können (Weyland \& Wittmann 2015, 2017; Weyland 2016, 2019; Weyland et al. 2019; Tramm \& Naeve-Stoß 2018; König \& Rothland 2018; Krasemann 2019):

- Parallelität von Forschen und Unterrichten (Dominanz des Unterrichtens)

- Forschendes Lernen (u. a. Akzeptanz, Abgrenzung zur Forschung, Qualifizierung der begleitenden Akteur*innen)

- Akteursvielfalt und deren Zusammenarbeit, curriculare Kohärenz (u. a. Organisation, Kommunikation der Professionalisierungserwartungen)

- Hochschulinterne Zusammenarbeit (u. a. fachspezifische Verständnisse)

Während die Studierenden besondere Belastungen durch das Forschende Lernen parallel zum Unterrichten äußern, was durch nicht wahrgenommene Bezüge zwischen denselben verschärft wird, wenden wissenschaftliche Beiträge dies in die Herausforderung, für Akzeptanz bei und eine Begriffsklärung zum Forschenden Lernen mit den Studierenden zu sorgen. Dies soll ebenfalls zwischen den Hochschullehrenden der unterschiedlichen Fächer erfolgen. Zudem 
wird diesbezüglich die Qualifizierung der schulischen Akteure als Aufgabe benannt. Darüber hinaus steckt aus Sicht der Hochschulen eher eine besondere Herausforderung in der mit der Akteursvielfalt verbundenen organisatorischen Kooperation sowie professionalisierungsbezogenen Kommunikation.

Allerdings resultieren die oben zusammengefassten Ziele und Herausforderungen, welche durchaus Überschneidungen aufweisen können, im Wesentlichen aus Ordnungsgrundlagen, Befragungen von Akteur*innen während bzw. nach der Durchführung des Praxissemesters sowie aus wissenschaftlichen Einschätzungen. Weitgehend unberücksichtigt sind bisher die Erwartungen, die im Rahmen des Entwicklungsprozesses von Studiengängen vorherrschen, gleichwohl in dieser Phase wesentliche Eckpunkte für die Durchführung und weitere Konkretisierung des Praxissemesters in Form von Begleitveranstaltungen sowie die darüber hinausgehenden Organisations- und Kooperationsformen festgelegt werden. Daher untersucht die vorliegende Studie die Erwartungen im Rahmen der Studiengangentwicklung. Damit rücken insbesondere diejenigen Akteursgruppen in den Blick, die sich aktiv in die Entwicklung von Studiengängen einbringen (sollten) (Wilbers \& Wittmann 2013). Da i. d. R. im Rahmen der Studiengangentwicklung mit nicht-kohärenten Ansprüchen bzw. Erwartungen der beteiligten Akteursgruppen zu rechnen ist (Wilbers \& Wittmann 2013) und dies vor dem Hintergrund der oben skizzierten Befunde auch mit Blick auf das Praxissemester zu erwarten ist, erscheint eine akteursgruppendifferenzierte Erfassung der Erwartungen angeraten, welche mit der vorliegenden Studie verfolgt wird. Die Erwartungen werden, der obigen $\mathrm{Zu}-$ sammenfassung des Forschungsstandes folgend, in die angestrebten Ziele sowie die erwarteten Herausforderungen bzw. Problemlagen unterschieden.

\section{Methode}

Zur Bearbeitung der oben angeführten Forschungsfrage wird auf leitfadengestützte qualitative Interviews zurückgegriffen. Diese sind Teil einer Fallstudie (Yin 2014), die den Prozess der reakkreditierungsbedingten Studiengangentwicklung des Masters für ein Lehramt an beruflichen Schulen an der ChristianAlbrechts-Universität (CAU) zu Kiel fokussiert. Darin wurde, dem Lehrkräftebildungsgesetz Schleswig-Holsteins (2014) folgend, die curriculare Integration eines Praxissemesters vorgenommen. Nach einer Einführungsveranstaltung und einem zweiwöchigen schulpraktischen Block im Sommersemester verbringen die Studierenden während des Wintersemesters grds. 14 Stunden pro Woche in den Schulen, wo sie im Wesentlichen von schulischen Mentor*innen betreut werden. Zudem besuchen die Studierenden hochschulische Begleitveranstaltungen insbesondere aus der Berufs- und Wirtschaftspädago- 
gik und ihrem allgemeinbildenden Unterrichtsfach zu bildungswissenschaftlichen und fachdidaktischen Fragestellungen mit engem Bezug zu Unterrichtshospitation, -planung, -durchführung und -reflexion sowie Forschendem Lernen. Ergänzend bietet das Landesseminar Berufliche Bildung Begleitveranstaltungen an. ${ }^{3}$

Die problemzentrierten Gruppen- und Einzelinterviews (Witzel \& Reiter 2012) wurden 2018/2019 im Rahmen des laufenden Dissertationsvorhabens von Immo Degner mit wesentlichen hochschulinternen Akteursgruppen der Studiengangentwicklung geführt. Hierzu zählen Studierende des Lehramts an beruflichen Schulen, Vertreter*innen des wissenschaftlichen Mittelbaus der Wirtschaftswissenschaften und der studierbaren Fachwissenschaften der allgemeinbildenden Unterrichtsfächer und ihrer Fachdidaktiken sowie Professor*innen dieser Fachwissenschaften und Fachdidaktiken (Wilbers \& Wittmann 2013). ${ }^{4}$ Zum Erhebungszeitpunkt war der Reakkreditierungsprozess so weit vorangeschritten, dass die Konzeption der Prüfungs- und Praktikumsordnung zum Praxissemester abgeschlossen und formal in Kraft getreten war, die Umsetzung des Praxissemesters ab Sommer/Herbst 2019 jedoch noch ausstand. Insofern verfügten die Interviewpartner*innen über keinerlei Erfahrungen aus der praktischen Implementation und Durchführung des selbigen im berufsbildenden Lehramt. Jedoch ist kritisch anzumerken, dass ein Großteil der hochschulischen Interviewpartner*innen aus den allgemeinbildenden Unterrichtsfächern zum Erhebungszeitpunkt Erfahrungen aus der zeitlich vorgelagerten Studiengangentwicklung mit curricularer Integration eines Praxissemesters im Lehramt für Gymnasien sowie seiner erstmaligen Durchführung ab dem Wintersemester 2018 aufweist, welches allerdings abweichende Gestaltungsmerkmale beinhaltet (z. B. achtwöchiger Schulpraxisblock, vier Forschungs-/Leitfragen anstelle von zwei $)^{6}$.

Für den vorliegenden Beitrag wurden diejenigen Interviewtranskripte sowie -auszüge herangezogen, die Aussagen zu der oben angeführten Forschungsfrage enthalten und folglich im inhaltlichen Zusammenhang mit dem oben skizzierten Forschungsstand (Zielen sowie Herausforderungen) stehen.

3 Eine detaillierte konzeptionelle Darstellung des Praxissemestermodells im Lehramt für berufliche Schulen an der CAU zu Kiel kann in diesem Beitrag leider nicht erfolgen. Einen ersten Einblick hierzu bietet das Material auf der Homepage der Abteilung Berufs- und Wirtschaftspädagogik der CAU zu Kiel: www.bwp.uni-kiel.de/de/studium-lehre/ praxissemester in Verbindung mit der dort verlinkten Prüfungs- und Praktikumsordnung.

4 Daneben zählen Wilbers und Wittmann $(2013,40)$ zu den internen Akteursgruppen der Studiengangentwicklung das Leitungspersonal sowie das Verwaltungspersonal, welches in der vorliegenden Studie vernachlässigt werden.

5 Vertreter*innen aus der Berufs- und Wirtschaftspädagogik (Mittelbau und Professoral) konnten in der Studie aus forschungsethischen Gründen leider nicht berücksichtigt werden.

6 Siehe zum Praxissemester im Lehramt für Gymnasien an der CAU zu Kiel: https://www.zfl.uni-kiel.de/de/schulpraktika/zwei-faecherpruefungsordnung-2017/Praxis semester. 
Damit liegt den folgenden Ergebnissen Datenmaterial von 16 Proband*innen aus 14 Interviews zugrunde. Hiervon sind sechs Gesprächspersonen der Gruppe der Studierenden des Lehramts an beruflichen Schulen (ST) zuzuordnen, vier der Gruppe der Vertreter*innen des wissenschaftlichen Mittelbaus (WM) (davon je zwei aus den Fachdidaktiken und Fachwissenschaften) sowie sechs der Gruppe der Professor*innen (P) (davon vier aus den Fachwissenschaften und zwei aus den Fachdidaktiken). Die Proband*innen wurden im Wesentlichen in Einzelinterviews befragt, um die individuellen Orientierungen und Erfahrungen rekonstruieren zu können (Nohl 2017). Da die Proband*innen aus der Gruppe der Studierenden in deutlich unterschiedlicher Intensität bei gleichzeitig ähnlich gelagerter Erfahrungsbasis am Studiengangentwicklungsprozess teilgenommen hatten, wurden zur stärkeren Konturierung der gruppenspezifischen Orientierungen, unter dem Ansinnen der Herstellung einer Selbstläufigkeit sowie Intensivierung der interaktiven Erzähldichte in den Gesprächen (Przyborski \& Wohlrab-Sahr 2014), neben zwei Einzelinterviews zwei Gruppeninterviews mit jeweils zwei Personen geführt. Die Auswertung der selektierten Interviewpassagen erfolgte mittels der inhaltlich-strukturierenden qualitativen Inhaltsanalyse (Kuckartz 2016; Mayring 2015).

\section{Tab. 1: Kategoriensystem der Ziele und Herausforderungen/Problemlagen}

\section{Oberkategorie: Ziele (Z)}

Z1 Förderung unterrichts- bzw. handlungspraktischer Fähigkeiten

Z2 Förderung (methodengeleiteter) theoretischer Reflexionsfähigkeit

Z3 Relationierung von Theorie und Praxis und die Relevanz von wissenschaftlichem Wissen erkennen

Z4 Anbahnung einer Forschenden Grundhaltung und Förderung von Forschungskompetenz

Z5 Reflexion der eigenen Kompetenzentwicklung und Berufswahlüberprüfung

Oberkategorie: Herausforderungen und Problemlagen $(\mathrm{H})$

H1 Überfrachtung/Überforderung aufgrund vielfältiger Aufgaben

H2 Herausforderungen im Zusammenhang mit Forschendem Lernen

H3 Herausforderungen im Zusammenhang mit der Begleitung und Reflexion des praktischen Handelns der Studierenden

H4 Herausforderungen aufgrund der Akteursvielfalt

Quelle: eigene Darstellung 
Das anfänglich auf der Basis des in Kapitel 2 dargestellten Forschungsstandes deduktiv-theoriegeleitet entwickelte Kategoriensystem wurde im Laufe der Analyse anhand des vorliegenden Datenmaterials und unter intensiver Diskussion im Forscherteam induktiv weiterentwickelt (Mayring 2015; Schreier 2014, Abs. 2). Die inhaltsanalytische Auswertung wurde durch die Software MAX QDA unterstützt. Tabelle 1 stellt das weiterentwickelte Ergebnis der Kategorienbildung dar.

\section{Darstellung und Diskussion der Ergebnisse}

\subsection{Darstellung und Diskussion der Ergebnisse zu den Zielen}

Förderung unterrichts- bzw. handlungspraktischer Fähigkeiten (Z1)

Die befragten Personen aus allen drei Akteursgruppen nennen das Ziel der Förderung unterrichts- bzw. handlungspraktischer Fähigkeiten durch ein Praxissemester, wobei dies insbesondere die Studierenden hervorheben (ST1, ST2, ST3, ST4, ST5, ST6). Dieses Ergebnis deckt sich mit jenen aus Evaluationsstudien zum Praxissemester (Weyland \& Wittmann 2017).

Die Studierenden verbinden mit dem Praxissemester u. a. die Hoffnung, dass die eigene Unterrichtskompetenz durch die Partizipation an den Erfahrungen der begleitenden Lehrkräfte gefördert wird. Daneben erhoffen sie sich, durch den Vollzug von praktischen Unterrichtshandlungen und der Teilhabe an Schulpraxis eine erste Basis zur Strukturierung und Organisation ihres späteren Lehrkräftealltages im Referendariat (und darüber hinaus) zu entwickeln (ST1, ST6). Wichtig sei hierbei die Erfahrung, direkt und persönlich vor Schüler*innen zu stehen (ST6). Interessant erscheint, dass die Studierenden neben dem Ausprobieren handlungs- und unterrichtspraktischer Fähigkeiten insbesondere das Erleben von Schulorganisation im „Kosmos Schule“ (ST3) bzw. „,im Alltag Schule“ (ST4) betonen (ST1, ST2, ST3, ST4).

Aus der Gruppe des wissenschaftlichen Mittelbaus wird der Erwerb handlungspraktischer Fähigkeiten damit begründet, möglichen Beschwerden von Lehrkräften bezüglich ,mangelnder Praxiserfahrung“ (WM1) der Studierenden vorzubeugen. Des Weiteren sehen sie in den unterrichtspraktischen Tätigkeiten eher ein Mittel zur Erreichung darüber hinausgehender Professionalisierungsziele. Diese können die Motivation der Studierenden bzgl. der Durchführung ihres Studiums erhöhen (WM3) und handlungsinitiierende Impulse bzw. Denkprozesse auslösen, die zu einer Reflexion über die eigene Profession anregen (WM1) (Ziel Z5). 
Es fällt auf, dass aus der Gruppe der Professor*innen lediglich eine Person eine Erwartung hinsichtlich der Zielkategorie Z1 formuliert. Diese äußert die Hoffnung, dass die Studierenden durch die Unterrichtserprobung besser auf das Referendariat vorbereitet werden (P4).

Förderung (methodengeleiteter) theoretischer Reflexionsfähigkeit (Z2)

Die Förderung der theoretischen Reflexionsfähigkeit wird ebenfalls von allen befragten Gruppen angeführt, wobei die Mehrheit und insbesondere die Studierenden dieses Ziel eher indirekt benennen (ST1, ST2, ST4, WM1, P3). Zudem bleibt offen, inwiefern diese Reflexion methodengeleitet erfolgen soll (vgl. Kap. 2).

Ein*e Proband*in des wissenschaftlichen Mittelbaus formuliert zwar nicht die Förderung der Reflexionsfähigkeit als Ziel, verweist jedoch auf die Notwendigkeit von theoretisch fundierten Reflexionsprozessen als Mittel: „Ich glaube, dass das nur wirklich sinnvoll ist, [...] wenn man das halt begleitet und wenn man so, ja, auch in Reflexionsprozesse kommt, das verbindet mit theoretischen Hintergrund, was man gelernt hat an der Uni“" (WM3, Abs. 85).

Relationierung von Theorie und Praxis und die Relevanz von wissenschaftlichem Wissen erkennen (Z3)

Im Vergleich zu den anderen Subzielkategorien benennen auffällig viele Proband*innen aller drei Akteursgruppen das Ziel der Relationierung von Theorie und Praxis sowie des Erkennens der Relevanz von wissenschaftlichem Wissen (ST1, ST2, ST3, ST5, ST6, WM1, WM2, WM3, P2, P3, P4, P6), worin ein weitgehender Konsens erkennbar wird.

Die Studierenden betonen, dass durch die direkte Auseinandersetzung mit der Schulpraxis ein Reflexionsprozess über das im Studium erworbene Wissen angestoßen und die Bedeutung dieses Wissens sowie ausgewählter Themenbereiche (z. B. Inklusion und Heterogenität) für die Praxis deutlich werde (ST2, ST3): ,in dem Moment ganz viel [verknüpft wird] [...] und [man] merkt, warum lernt man dies, warum lernt man das“ (ST1). Das im Studium „stur in meinen Kopf geprügelt[e]" (ST3) Wissen werde in der Praxis tatsächlich angewandt, überdacht und könne zukünftig gezielt eingesetzt werden. Zudem sehen Studierende die Möglichkeit, dass sich die Lehrveranstaltungen an der Universität verbessern könnten, da weitere Erfahrungswerte durch die Studierenden eingebracht werden können (ST6).

Aus dem wissenschaftlichen Mittelbau benennt ein*e Proband*in die „Verknüpfung von universitärem Herangehen und der Praxis“(WM3, Abs. 87) explizit und mehrfach als Ziel. 
Aus der Gruppe der Professor*innen führt eine Person an: „Aber einmal das, was ich als wissenschaftlich fundiertes Wissen im ersten- in der ersten Phase, in einem Seminar, in einer Praktikumsvorbereitung erarbeitet habe, kennen gelernt habe, nun einmal in der Praxis selbst zu beobachten, zu evaluieren, zu diagnostizieren, zu schauen, wie weit die Messinstrumente reichen, die wir haben, zu sehen, wie beispielsweise Lernleistungen bewertet werden [...] wir wollen mal schauen, [...] [dass wir das,] was wir im Studium machen, vielleicht in die Schulen [...] bringen [können], ohne dass wir alles besser wissen, keineswegs“" (P3, Abs. 86). Neben der Anwendung von theoretischem Wissen gehe es darum, dieses Wissen auch vor dem Hintergrund der Praxis bzw. der Erfahrungen in der Praxis zu reflektieren.

Die Aussagen der Proband*innen deuten zudem darauf hin, dass neben dem auf die Professionalisierung der Studierenden bezogenen Ziel auch Potenziale für die Universitäts- sowie Schulentwicklung gesehen werden (WM1, WM3, P1, P3, P6). So führt bspw. ein*e Proband*in aus der Gruppe der Professor*innen an, dass für die Schule die Möglichkeit bestehe, ,reinzuschnuppern, in das, was in der Universität passiert und die Universität umgekehrt, weil sie eine Chance hat, besser kennen zu lernen, was in der Praxis passiert" (P6, Abs. 123).

Anbahnung einer Forschenden Grundhaltung und Förderung von Forschungskompetenz (Z4)

Diese Subzielkategorie wird relativ wenig durch die Proband*innen benannt (P3, WM2, WM3, ST4, ST3). Dies ist insbesondere vor dem Hintergrund der in der Literatur hervorgehobenen Bedeutung des Forschenden Lernens im Praxissemester (Weyland et al. 2019, 14) erstaunlich. Andererseits ist anzumerken, dass in der Erhebung nicht explizit nach dem Forschenden Lernen und den damit verfolgten Zielen gefragt wurde.

Die Studierenden können dieses Ziel durchaus nachvollziehen, ohne dass sie darin jedoch einen Sinn für sich sehen, da das Praxissemester durch „,so ein bisschen forschen“ (ST3, Abs. 105) unnötig ,aufgebläht wird“ (ST3, Abs. 168) (s. u. Herausforderung H2).

Aus der Gruppe des wissenschaftlichen Mittelbaus wird die Förderung der Forschungskompetenz bei den Studierenden als zu verfolgendes Ziel angesprochen (WM2, WM3). Hierbei wird das Ziel jedoch insbesondere unter dem Aspekt der Verknüpfung von Theorie und Praxis sowie in Zusammenhang mit der Bearbeitung einer Forschungsaufgabe diskutiert.

Ein*e Proband*in aus der Gruppe der Professor*innen sieht einen Kernpunkt des Praxissemesters mit dem Potenzial für nachhaltige Effekte darin, dass die Studierenden die Praxis vor dem Hintergrund einer ,relativ eng zuge- 
schnittenen Forschungsfrage" (P3, Abs. 86) sowie dem wissenschaftlich fundierten Wissen aus dem Studium analysieren und gestalten, und nicht unhinterfragt tradierte Handlungspraktiken von anderen übernehmen (P3). Dies deutet auf das Ziel der Entwicklung einer Forschenden Grundhaltung in der Praxis hin, welche eine Nähe zum Ziel der Relationierung von Theorie und Praxis (siehe Z3) erkennen lässt.

Reflexion der eigenen Kompetenzentwicklung und Berufswahlüberprüfung (Z5)

Unter den drei Akteursgruppen herrscht große Einigkeit darüber, dass das Praxissemester der Berufswahlüberprüfung bzw. -reflexion der Studierenden in Verbindung mit der Berufsfelderkundung dienen kann (ST1, ST2, ST3, ST6, P1, P2, P5, WM1, WM2, WM3, WM5).

In diesem Rahmen erhoffen sich die Studierenden Klarheit hinsichtlich ihrer eigenen Kompetenzen bzw. Stärken und Schwächen sowie Entwicklungsbedarfe (ST1, ST2, ST6), bspw. darüber „,was klappt, was klappt vielleicht nicht so gut, was muss ich an mir noch ändern“(ST6, Abs. 174) und „was man alles doch nicht weiß und was man vielleicht noch bräuchte" (ST1, Abs. 192). Ein*e Proband*in aus der Studierendengruppe bemerkt, dass insbesondere längere semesterbegleitende Praxisphasen ein „repräsentativeres Bild“ (ST4) eines schulischen Berufsalltags ermöglichen könnten, da sich die Schüler*innen an sie gewöhnen und sich ihnen gegenüber normal verhalten könnten. Vor dem Hintergrund einer ggf. korrigierten Einschätzung zum Lehrerberuf und zum Vorbereitungsdienst (ST2) könnten sie sich ,intensiv mit ihrer persönlichen Weiterentwicklung" (ST1) beschäftigen.

Der wissenschaftliche Mittelbau betont die Bedeutung für die „Berufserkundung“ (WM2) in der Form, dass sich die Studierenden ,ein bisschen mehr in die Rolle" (WM3) der Lehrkraft hineinfinden könnten und daneben die Möglichkeit hätten zu reflektieren, ,was gehört denn alles noch so dazu, neben dem Unterricht" (WM5, Abs. 90).

Auch die Professor*innen betonen eine mögliche Steigerung der Selbstreflexionsfähigkeit der Studierenden in Bezug auf das Berufsfeld einer Lehrkraft an einer Schule und eine Überprüfung des Berufswunsches (P1, P5). Daneben merken sie an, dass der Zeitraum für eine abschließende Berufswahlreflexion möglicherweise nicht ausreiche. Dies begründen sie damit, dass den Studierenden im Rahmen des polyvalenten Studiengangs neben einem Lehramt an beruflichen Schulen weiterhin berufliche Perspektiven in betrieblichen Kontexten offenstehen, was wiederum die Berufswahlentscheidung komplexer gestalte (P2). 


\section{Zusammenfassende Diskussion der Ziele}

Das Datenmaterial zeigt, dass die hochschulinternen Akteur*innen im Rahmen des Studiengangentwicklungsprozesses die aus dem Forschungsstand (Kap. 2) bekannten, auf die Professionalisierung der Studierenden bezogenen Ziele ebenfalls weitgehend verfolgen, jedoch mit unterschiedlicher Intensität und Gewichtung.

Die Studierenden betonen den Erwerb von unterrichts- und handlungspraktischen Fähigkeiten. Dieses Ziel wird von Seiten der Professor*innen sowie des wissenschaftlichen Mittelbaus nicht explizit formuliert. Vielmehr wird kritisch angemerkt, dass die universitäre Lehrerbildung eben nicht im Sinne einer klassischen betrieblichen Ausbildung dazu führen sollte, dass repetitive „Rezepte“ (P4, Abs.70) erlernt werden. Die Lehrenden an der Hochschule heben die Relationierung von Theorie und Praxis sowie die Berufswahlüberprüfung bzw. -reflexion hervor. Andererseits formulieren sie beinahe keine Ziele in Hinblick auf die Herausbildung einer Forschenden Grundhaltung. Dieses Ergebnis erstaunt, da im wissenschaftlichen Diskurs zu verlängerten Praxisphasen die Anbahnung einer Forschenden Grundhaltung zumeist als eine „wesentliche Zielsetzung“ (Weyland et al. 2019, 14) benannt wird. Die Förderung methodengeleiteter theoretischer Reflexionsfähigkeit wird als Ziel des Praxissemesters nicht direkt benannt, was jedoch auch an der großen Nähe desselben zur Zielkategorie Z3 (Theorie-Praxis-Relationierung) liegen kann.

Neben den Erwartungen, die in Verbindung mit der Professionalisierung von Studierenden stehen, benennen die Akteur*innen noch weitere Ziele. Hierzu zählen insbesondere die Schul- und Universitätsentwicklung, die sowohl von Hochschuldozierenden wie auch von Studierenden benannt werden: Beispielsweise könnten die Erfahrungen und Erkenntnisse der Studierenden aus dem Praxissemester in die Weiterentwicklung der universitären Lehrveranstaltungen einfließen (ST6). Daneben könnten die betreuenden Lehrkräfte wissenschaftliche Erkenntnisse für ihre Schulen gewinnen und Reflexionsmomente für den eigenen Unterricht erhalten (WM3, WM1, P6).

Zudem weisen alle drei Akteursgruppen auf die Möglichkeit hin, die beiden Phasen der Lehrerausbildung besser miteinander zu verzahnen (WM1, P6, ST1). Dies ist ein Ziel, welches durchaus auch im wissenschaftlichen Diskurs zu finden ist (Pätzold 2018, 52).

\subsection{Darstellung und Diskussion der Ergebnisse zu Herausforderungen und Problemlagen}

Die befragten Akteursgruppen und die einzelnen Proband*innen benennen mögliche Herausforderungen und Problemlagen in unterschiedlichster Art, was eine Zuordnung zu den aus dem Forschungsstand gewonnenen Kategorien 
erschwert. Daher wurden diese im Rahmen der Datenauswertung induktiv überarbeitet und angepasst.

Überfrachtung bzw. Überforderung aufgrund vielfältiger Aufgaben (H1)

Einige Studierende fürchten eine Überfrachtung im Praxissemester, die sie mit den damit verbundenen vielfältigen Aufgaben begründen (ST1, ST2, ST4, ST5, ST6). Deutlich wird die aus dem Forschungsstand bereits bekannte wahrgenommene Überfrachtung des Praxissemesters durch die Parallelität von Forschen und Unterrichten in Verbindung mit einer nachgeordneten Priorität zum Forschen (Weyland 2019; Brenneke et al. 2018; König \& Rothland 2018). So führt ein*e Student*in an, die Beantwortung einer Forschungsfrage im Sinne des Forschenden Lernens werde „nebenbei nicht so klappen“ (ST6, Abs. 186). Zudem sprechen die befragten Studierenden weitere Gründe einer befürchteten allgemeinen Überforderung an: Neben den parallel zu bewältigenden Aufgaben in der Schule sowie in der Universität (ST4, ST6) wird eine zeitliche Herausforderung in dem befürchteten Zurücklegen weiter Wegstrecken zwischen Universität und Schule benannt (ST5). Studierende erwarten hohe körperliche Belastung durch diese vielfältigen Aufgaben (ST4) sowie aus der Verbindung von privaten Herausforderungen (z. B. Nebenjobs) mit der Praktikumsphase (ST6). Ein*e Proband*in spitzt dies in einer abschließenden Bewertung als Rückentwicklung des Studiums für manche Studierende zu (ST1).

Während aus der Gruppe des wissenschaftlichen Mittelbaus kein*e Proband*in diesbezüglich mögliche Herausforderungen bzw. Problemlagen äuBert, merkt eine Person aus der Gruppe der Professor*innen kritisch an, dass die Arbeitsbelastung für die Studierenden zu hoch sein könnte, weshalb die Lehrenden an der Hochschule in der Implementierungsphase möglicherweise „nachsteuern“ (P6, Abs. 123) müssten.

Herausforderungen im Zusammenhang mit Forschendem Lernen (H2)

Neben der oben angeführten Überfrachtung des Praxissemesters werden aus den Aussagen einzelner Studierender weitere Herausforderungen im Zusammenhang speziell mit dem Forschenden Lernen deutlich. Ein*e Proband*in führt an, dass erst einmal der „Schulschock“ (ST3) überwunden werden sollte, bevor dort geforscht werden könne. Dies deutet darauf hin, dass der Sinn des Forschenden Lernens dem Studierenden noch nicht klargeworden ist, was wiederum zu mangelnder Akzeptanz dieses Ziel beiträgt (s. o. Ziel Z4). Ähnlich sehen Studierende auch während oder nach der Durchführung des Praxissemesters vielfach keinen Sinn in dem Forschenden Lernen und den damit verfolgten Zielen (Weyland 2019; Weyland et al. 2019). 
Aus dem wissenschaftlichen Mittelbau sowie der Gruppe der Professor*innen wird auf einen deutlichen Unterschied zwischen wissenschaftlicher und studentischer Forschung im Praxissemester verwiesen und dass eine Herausforderung in der klaren begrifflichen Definition dieser Forschungsformen sowie einer damit einhergehenden klaren Bedeutungsabgrenzung bestehe (WM3; P5).

Herausforderungen im Zusammenhang mit der Begleitung und Reflexion des praktischen Handelns der Studierenden (H3)

Alle drei Akteursgruppen sehen mögliche Herausforderungen im Zusammenhang mit der allgemeinen Begleitung der Studierenden sowie insbesondere bezüglich ihrer unterrichts- und handlungspraktischen Fähigkeiten (ST2, ST3, WM3, P1, P3, P5, P6).

Studierende weisen auf eine mögliche Unerfahrenheit der betreuenden Lehrkräfte in Hinblick auf die Ausbildung von Nachwuchslehrkräften hin (ST3). Ebenfalls mit Blick auf die schulischen Lehrkräfte wird aus der Gruppe der Professor*innen das möglicherweise unzureichende Wissen über die Aufgaben eines*r Mentor*in im Praxissemester problematisiert (P6). Zudem befürchten zwei Proband*innen eine unklare Kommunikation zwischen den Studierenden und den schulischen Mentor*innen, welche zur Unklarheit über die abzuleistenden Praktikumsanforderungen führen könne (ST2, P6).

Jeweils ein*e Proband*in aus dem wissenschaftlichen Mittelbau sowie der Professor*innen betont die notwendige stetige Reflexion durch die Studierenden (WM3, P3, s.o. Ziel Z2), ohne die der Nutzen der Praxisphase in Frage gestellt wird: ,[W]enn nicht reflektiert wird, was passiert, glaube ich, dass [die Praxisphase] nicht effektiv ist" (WM3, Abs. 85). Wer für die Reflexion bzw. ihre Anregung und Begleitung verantwortlich ist und wie diese erfolgen sollte, führen die Proband*innen nicht weiter aus.

Zudem weisen Professor*innen auf die Gefahr des Modell- und Imitationslernens der Studierenden in den Schulen hin (P1, P3). Problematisch daran sei u. a., dass die schulischen Betreuer*innen die aus der Hochschullehre vermittelten Theorien zur Unterrichtsgestaltung als nicht umsetzbar erachten (P1) oder tradierte didaktische Modelle unreflektiert an die Studierenden vermittelt werden (P3). Ebenfalls wird auf die bereits bestehenden Belastungen der schulischen Mentor*innen hingewiesen, welche möglicherweise eine intensive Auseinandersetzung mit der Professionalisierung der Studierenden hemmt (P1). 
Herausforderungen aufgrund der Akteursvielfalt (H4)

Einzelne Proband*innen benennen Herausforderungen, die im Kontext der Zusammenarbeit der am Praxissemester beteiligten Institutionen entstehen können (ST1, ST2, WM1, WM3, P6).

Manche Studierende offenbaren, dass ihnen die Funktion und Verantwortlichkeit der Lehrenden an den beiden Lernorten (Universität und Schule) nicht eindeutig klar sei (ST1, ST2).

Ein*e Proband*in aus der Gruppe des wissenschaftlichen Mittelbaus sieht ein mögliches Abstimmungsproblem zwischen Schulen und Universität in Hinblick auf die jeweils an die Studierenden herangetragenen Leistungsanforderungen (WM3). Zudem könne es mögliche Überschneidungen von Präsenzterminen an der Hochschule und dem Praktikumseinsatz in der Schule geben, was wiederum für die Studierenden eine organisatorische Belastung darstellen (WM1) und letztendlich zu deren Überforderung beitragen könnte (siehe H2).

Daneben problematisieren ein*e Professor*in und ein*e Vertreter*in des wissenschaftlichen Mittelbaus, dass es zwischen hochschulischen und schulischen Akteur*innen unterschiedliche Vorstellungen über die Ausbildung der Studierenden in den Schulen geben kann. Dies könne sich u. a. in der Form zeigen, dass Studierende im Praxissemester nicht vorrangig eine reflexive theoriegeleitete Auseinandersetzung mit der Praxis durchführen, sondern vermehrt als Vertretungslehrer*innen eingesetzt werden (P6, WM3). Eine Folge dieser beispielhaft skizzierten missglückten Kommunikation der universitären sowie schulischen Akteur*innen über die Professionalisierungserwartung der Studierenden kann ein ausgeprägter „frustrierende[r] Eindruck von Schule“ (P6, Abs. 125) bei den potentiellen Nachwuchslehrkräften sein. Zusammenfassend sehen die Lehrenden an der Hochschule Herausforderungen im Zusammenhang mit der Zusammenarbeit der verschiedenen Akteure vorrangig als Problem mit bzw. in der anderen Institution, d. h. der Schule.

\section{Zusammenfassende Diskussion der Herausforderungen und Problemlagen}

Das Datenmaterial zeigt, dass die antizipierten Herausforderungen und Problemlagen zwischen den befragten Akteursgruppen sehr unterschiedlich ausfallen. Während die Mehrheit der Studierenden die größten Herausforderungen in der Überforderung bzw. Überlastung sehen, teilt die Mehrheit der Gruppen der hochschulischen Lehrenden diese Befürchtung nicht. Hingegen sieht die Mehrheit der Professor*innen eine Herausforderung in der zielgerichteten Betreuung der Studierenden im Schulpraktikum sowie in der damit verbundenen theoriegeleiteten Reflexion des praktischen Handelns. Es fällt auf, dass aus dem wissenschaftlichen Mittelbau sehr wenig Herausforderungen bzw. Problemlagen im Zusammenhang mit dem Praxissemester benannt werden. 
Darüber hinaus führen einzelne Akteur*innen weitere Herausforderungen und Problemlagen an. Beispielsweise wird die angedachte Lage des Praxissemesters im Masterstudiengang kritisch diskutiert (P1, P4, ST3, ST6, WM3). Ähnlich wie im wissenschaftlichen Diskurs bemängeln Professor*innen fehlende empirische Befunde über die Wirksamkeit der Verlängerung von Praxisphasen und zwar konkret in Hinblick auf die Förderung der praktischen Unterrichtsund Handlungsfähigkeit bei Studierenden (P3) und dass die Zielerreichung aufwendig sowie mangels der Befunde ungewiss sei (P1) (Frommberger \& Lange 2018; König \& Rothland 2018; Leonhard \& Herzog 2018; Weyland \& Wittmann 2015, 2017, 2019).

\section{Fazit und Ausblick}

Bezogen auf die eingangs gestellte Forschungsfrage, welche Erwartungen wesentliche Akteursgruppen im Rahmen der Studiengangentwicklung mit der Einführung eines Praxissemesters in das Studium für ein Lehramt an beruflichen Schulen verbinden, kann in der vorliegenden Studie ein durchaus heterogenes Bild zwischen Studierenden, Vertreter*innen des wissenschaftlichen Mittelbaus und Professor*innen festgestellt werden. In den festgestellten Erwartungsdiskrepanzen zwischen den Akteursgruppen kann eine ähnlich große Zielvielfalt ausgemacht werden, wie sie im bisherigen Forschungsstand zu finden und wie sie grundsätzlich auch für Prozesse der Studiengangentwicklung üblich ist (siehe Kap. 2). Zudem zeigt sich bei den im Kontext der Studiengangentwicklung befragten Studierenden, ähnlich wie bei den im Rahmen von durchgeführten Praxissemestern befragten Studierenden, eine Fokussierung auf unterrichtspraktische Fähigkeiten, während die Lehrenden an der Hochschule die Relationierung von Theorie und Praxis sowie die Berufswahlüberprüfung bzw. -reflexion hervorheben. Andererseits erstaunt das in Anbetracht der beachtlichen wissenschaftlichen Diskussion nicht explizit benannte Ziel der forschenden Grundhaltung. Auch hinsichtlich der Herausforderungen zeigt sich eine breite Spanne sowie eine Diskrepanz zwischen Studierenden und Hochschullehrenden. Während - ähnlich wie in Befragungen im Rahmen der Durchführung von Praxissemestern - Studierende insbesondere Überforderungs- bzw. Überlastungsängste äußern, sehen die Professor*innen eine Herausforderung in der zielgerichteten Betreuung der Studierenden im Schulpraktikum sowie in der damit verbundenen theoriegeleiteten Reflexion des praktischen Handelns.

In dieser Studie nicht erhoben werden konnte, wie mit diesen unterschiedlichen Erwartungen im Rahmen der Studiengangentwicklung umgegangen werden kann bzw. soll und wie diese in die Konzeption und Implementation des Praxissemesters Eingang erhalten. In diesem Zusammenhang wäre auch 
die Vereinbarkeit der unterschiedlichen Zielsetzungen mit Blick auf das Ziel der Professionalisierung der angehenden Lehrkräfte kritisch zu reflektieren und zu diskutieren (bspw. die singuläre Fokussierung auf unterrichtspraktische Fähigkeiten) sowie wie mit diesen im Prozess der Studiengangentwicklung und im Anschluss daran in der regelmäßigen Durchführung des Praxissemesters bspw. im Rahmen der Begleitveranstaltungen umzugehen ist. Hierbei erscheint zum einen eine Verständigung über das Professionalisierungsverständnis und/oder mögliche unterschiedliche Teilziele mit ihren jeweiligen kritisch $\mathrm{zu}$ reflektierenden Berechtigungen zwischen hochschulischen Akteur*innen und Studierenden sowie zwischen den hochschulischen Akteur*innen selbst als grundlegend. Zum anderen könnten bereits während der Entwicklungsphase des Studienganges, insbesondere in Hinblick auf die schulpraktischen Phase sowie der Begleitveranstaltungen, die Verhinderung von bzw. Lösungsansätze zu möglichen antizipierten Überlastungen und Überforderungen der Studierenden im Kontext des Praxissemesters diskutiert werden, um einer etwaigen Frustration oder Abwehrhaltung der Studierenden und der bereits belegten möglichen Angebots-Nutzungsdiskrepanz (Naeve-Stoß 2013) zu begegnen. Vor dem Hintergrund der oben skizzierten Ergebnisse erscheint auch ein frühzeitiger Einbezug der schulischen Lehrkräfte in diese Diskussions- und Reflexionsprozesse angeraten (bspw. damit Studierende nicht, entgegen des konzeptionell verfolgten Professionalisierungsgedankens, lediglich als Vertretungslehrkräfte eingesetzt werden). Hierbei sollten die Ziel- bzw. Nutzungsvorstellungen der am Praxissemester beteiligten Akteure bestenfalls bereits vor der praktischen Umsetzung des selbigen transparent und abgestimmt sein, um mögliche Wirkungsverluste zu vermeiden.

Forschungsmethodisch bleibt einschränkend anzumerken, dass mit der Anlage dieser Studie kein Anspruch auf generalisierbare Aussagen erhoben wird. Vielmehr sollen erste relevante Einblicke in die Erwartungshaltung der unterschiedlichen Akteursgruppen im Kontext der Studiengangentwicklung gegenüber dem Praxissemester gegeben werden.

Für zukünftige Forschung wäre es inhaltlich interessant und weiterführend, im Rahmen einer Praxissemesterevaluation zu untersuchen, welche Bewertung die jeweiligen Akteursgruppen hinsichtlich der tatsächlichen Verfolgung und/oder Erreichung der im Weiterentwicklungsprozess formulierten Ziele vornehmen und inwiefern diese Ziele konstant bleiben oder sich verändern. 


\section{Literatur}

Brenneke, B., Pfaff, N., Schrader, T.-B. \& Tervooren, A. (2018). Das Praxissemester als Erfahrungsraum Forschenden Lernens? Ambivalenzen in der Aneignung qualitativer Forschungsmethoden. In M. Artmann, M. Berendonck, P. Herzmann \& A. Liegmann (Hrsg.), Professionalisierung in Praxisphasen der Lehrerbildung. Qualitative Forschung aus Bildungswissenschaft und Fachdidaktik (S. 38-55). Bad Heilbrunn: Verlag Julius Klinkhardt.

Frommberger, D. \& Lange, S. (2018). Zur Ausbildung von Lehrkräften für berufsbildende Schulen. Befunde und Entwicklungsperspektiven. Working Paper Forschungsförderung Nummer 060. Düsseldorf: Hans-Böckler-Stiftung.

Gröschner, A. \& Hascher, T. (2019). Praxisphasen in der Lehrerinnen- und Lehrerbildung. In M. Harring, C. Rohlfs \& M. Gläser-Zikuda (Hrsg.), Handbuch Schulpädagogik (S. 652-664). Münster: Waxmann.

Gröschner, A., Schmitt, C. \& Seidel, T. (2013). Veränderung subjektiver Kompetenzeinschätzungen von Lehramtsstudierenden im Praxissemester. Zeitschrift für Pädagogische Psychologie, 27(1/2), 77-86.

Großmann, K., Bach, A. \& Winkel, J. (2017). Das Praxissemester in Flensburg. In R. Schüssler, A. Schöning, V. Schwier, S. Schicht, J. Gold \& U. Weyland (Hrsg.), Forschendes Lernen im Praxissemester. Zugänge, Konzepte, Erfahrungen (S. 8187). Bad Heilbrunn: Verlag Julius Klinkhardt.

König, J. \& Rothland, M. (2018). Das Praxissemester in der Lehrerbildung: Stand der Forschung und zentrale Ergebnisse des Projekts Learning to Practice. In J. König, M. Rothland \& N. Schaper (Hrsg.), Learning to Practice, Learning to Reflect? Ergebnisse aus der Längsschnittstudie LtP zur Nutzung und Wirkung des Praxissemesters in der Lehrerbildung (S. 1-62). Wiesbaden: Springer Fachmedien.

Krasemann, B. (2019). ,Jetzt am Ende des Praxissemesters verfüge ich über mehr Autorität und habe kein Problem damit, ab und zu ein Machtwort auszusprechen." Zur Involviertheit Studierender in Praxisphasen. In J. Kosinár, A. Gröschner \& U. Weyland (Hrsg.), Langzeitpraktika als Lernräume. Historische Bezüge, Konzeptionen und Forschungsbefunde (S. 71-85). Münster und New York: Waxmann.

Kuckartz, U. (2016). Qualitative Inhaltsanalyse. Methoden, Praxis, Computerunterstützung. Weinheim und Basel: Beltz Juventa.

Lange, S. \& Frommberger, D. (2017). Zur Ausgestaltung schulischer Praxisphasen im beruflichen Lehramtsstudium - Ergebnisse einer ersten Analyse. In J. Seifried, B. Ziegler \& S. Seeber (Hrsg.), Jahrbuch der berufs- und wirtschaftspädagogischen Forschung 2017 (S. 113-127). Opladen u. a.: Verlag Barbara Budrich.

Land Schleswig-Holstein (2014). Lehrkräftebildungsgesetz Schleswig-Holstein. LehrBG vom 15. Juli 2014. Online: http://www.gesetze-rechtsprechung.sh.juris.de/jportal/?quelle=jlink\&query $=\mathrm{LehrBiG}+\mathrm{SH} \& \mathrm{psml}=$ bsshoprod.psml\&max $=$ true (12.12.2019).

Leonhard, T. \& Herzog, S. (2018). Was Langzeitpraktika leisten (können) - empirische und konzeptionelle Erkundungen. In M. Rothland \& N. Schaper (Hrsg.), Forschung zum Praxissemester in der Lehrerbildung (S. 5-23). Landau/Pfalz: Verlag Empirische Pädagogik.

Mayring, P. (2015). Qualitative Inhaltsanalyse. Grundlagen und Techniken. Weinheim und Basel: Beltz. 
Mertens, S., Schlag, S. \& Gräsel, C. (2018). Die Bedeutung der Berufswahlmotivation, Selbstregulation und Kompetenzselbsteinschätzungen für das bildungswissenschaftliche Professionswissen und die Unterrichtswahrnehmung angehender Lehrkräfte zu Beginn und am Ende des Praxissemesters. In M. Rothland \& N. Schaper (Hrsg.), Forschung zum Praxissemester in der Lehrerbildung (S. 66-84). Landau/Pfalz: Verlag Empirische Pädagogik.

Naeve-Stoß, N. (2013). Studienreform aus studentischer Perspektive. Einzelfallstudien zur Rekonstruktion studentischer Wahrnehmungen, Beurteilungen und Studienstrategien im Rahmen des Lehramtsstudiums für berufliche Schulen. Paderborn: Eusl-Verlagsgesellschaft mbH.

Naeve-Stoß, N. \& Tramm, T. (2017). Forschendes Lernen im Hamburger Kernpraktikum. In R. Schüssler, A. Schöning, V. Schwier, S. Schicht, J. Gold \& U. Weyland (Hrsg.), Forschendes Lernen im Praxissemester. Zugänge, Konzepte, Erfahrungen (S. 88-95). Bad Heilbrunn: Verlag Julius Klinkhardt.

Nohl, A.-M. (2017). Interview und Dokumentarische Methode. Anleitungen für die Forschungspraxis. Wiesbaden: Springer VS.

Pätzold, G. (2018). Wirksame Lehrerbildung verknüpft Theorie und Praxis. Wirtschaft \& Erziehung, 70(2), 51-56.

Przyborski, A. \& Wohlrab-Sahr, M. (2014). Qualitative Sozialforschung. Ein Arbeitsbuch. München: Oldenbourg Wissenschaftsverlag GmbH.

Schreier, M. (2014). Varianten qualitativer Inhaltsanalyse: Ein Wegweiser im Dickicht der Begrifflichkeiten. Forum Qualitative Sozialforschung, 15(1), Art. 18. Online: http:/www.qualitative-research.net/index.php/fqs/article/download/2043/3636 (12.12.2019).

Schubarth, W. (2017). Lehrerbildung in Deutschland. Sieben Thesen zur Diskussion. In W. Schubarth, S. Mauermeister \& A. Seidel (Hrsg.), Studium nach Bologna. Befunde und Positionen (S. 127-136). Potsdam: Universitätsverlag Potsdam.

Schüssler, R. \& Weyland, U. (2014). Praxissemester - Chance zur Professionalitätsentwicklung. In R. Schüssler, V. Schwier, G. Klewin, S. Schicht, A. Schöning \& U. Weyland (Hrsg.), Das Praxissemester im Lehramtsstudium: Forschen, Unterrichten, Reflektieren (S. 23--42). Bad Heilbrunn: Verlag Julius Klinkhardt.

Seifert, A. \& Schaper, N. (2018). Die Veränderung von Selbstwirksamkeitserwartungen und der Berufswahlsicherheit im Praxissemester. Empirische Befunde zur Bedeutung von Lerngelegenheiten und berufsspezifischer Motivation der Lehramtsstudierenden. In J. König, M. Rothland \& N. Schaper (Hrsg.), Learning to Practice, Learning to Reflect? Ergebnisse aus der Längsschnittstudie LtP zur Nutzung und Wirkung des Praxissemesters in der Lehrerbildung (S. 195-222). Wiesbaden: Springer Fachmedien.

Seifried, J. \& Wuttke, E. (2017). Weiterentwicklung professioneller Kompetenzen von Lehrkräften an beruflichen Schulen. In B. Bonz, H. Schanz \& J. Seifried (Hrsg.), Berufsbildung vor neuen Herausforderungen. Wandel von Arbeit und Wirtschaft (S. 177-194). Baltmannsweiler: Schneider Hohengehren.

Tramm, T. \& Naeve-Stoß, N. (2018). Praxisphasen im Lehramtsstudium: Es kommt darauf an, was man daraus macht. Erfahrungen mit dem Kernpraktikum an der Universität Hamburg. In J. Schlicht \& U. Moschner (Hrsg.), Berufliche Bildung an der Grenze zwischen Wirtschaft und Pädagogik. Reflexionen aus Theorie und Praxis (S. 285-306). Wiesbaden: Springer VS. 
Wilbers, K. \& Wittmann, M. (2013). Strategisches Management von Studiengängen an Universitäten. In K.-H. Gerholz \& P. F. E. Sloane (Hrsg.), Studiengänge entwickeln-Module gestalten. Eine Standortbestimmung nach Bologna (S. 31-50). Paderborn: Eusl-Verlagsgesellschaft.

Witzel, A. \& Reiter, H. (2012). The Problem-Centred Interview. London: Sage Publications.

Weyland, U. (2016). Schulpraktische Studien im Fokus des Lehramtsstudiums. Forschendes Lernen im Praxissemester als erfolgreiche Formel für eine bessere Lehrerbildung? Die berufsbildende Schule, 68(11/12), 380-387.

Weyland, U. (2019). Forschendes Lernen in Langzeitpraktika - Hintergründe, Chancen und Herausforderungen. In M. Degeling, N. Franken \& S. Freund (Hrsg.), Herausforderung Kohärenz: Praxisphasen in der universitären Lehrerbildung. Bildungswissenschaftliche und fachdidaktische Perspektiven (S. 25-64). Bad Heilbrunn: Verlag Julius Klinkhardt.

Weyland, U., Gröschner, A. \& Kosinár, J. (2019). Langzeitpraktika en vogue - Einführung in den Themenschwerpunkt. In J. Kosinár, A. Gröschner \& U. Weyland (Hrsg.), Langzeitpraktika als Lernräume. Historische Bezüge, Konzeptionen und Forschungsbefunde (S. 7-25). Münster und New York: Waxmann.

Weyland, U. \& Wittmann, E. (2011). Zur Einführung von Praxissemestern: Bestandsaufnahme, Zielsetzungen und Rahmenbedingungen. In U. Faßhauer, B. Fürstenau \& E. Wuttke (Hrsg.), Grundlagenforschung zum Dualen System und Kompetenzentwicklung in der Lehrerbildung (S. 49-60). Opladen: Verlag Barbara Budrich.

Weyland, U. \& Wittmann, E. (2015). Langzeitpraktika in der Lehrerausbildung in Deutschland - Stand und Perspektiven. journal für lehrerInnenbildung, 15(1), 821.

Weyland, U. \& Wittmann, E. (2017). Praxissemester en vogue. In R. Schüssler, A. Schöning, V. Schwier, S. Schicht, J. Gold \& U. Weyland (Hrsg.), Forschendes Lernen im Praxissemester. Zugänge, Konzepte, Erfahrungen (S. 17-29). Bad Heilbrunn: Verlag Julius Klinkhardt.

Yin, R. K. (2014). Case study research. Design and methods. Los Angeles: SAGE Publications, Inc. 



\title{
Studienzufriedenheit und Studienerfolg im ersten Studienjahr - Studierende im Lehramt an beruflichen Schulen und an Gymnasien im Vergleich
}

\author{
Josephine Berger und Birgit Ziegler
}

\section{Theoretische Rahmung und Erkenntnisperspektive}

Prozesse und Ergebnisse beruflicher Bildung resultieren aus der Vermittlung von Selektion und Professionalisierung. Davon ist die Lehrerkräfteausbildung nicht ausgenommen, sondern die Thematik ist hier wegen gewisser Spezifika sowie der gesellschaftlichen Schlüsselstellung dieses Berufs (z. B. Ziegler 2019, 531ff.) besonders virulent. Aus diesem Grund wurde vermutlich in der ersten Ausschreibung der Qualitätsoffensive Lehrerbildung durch das BMBF die „Eingangsdiagnostik und Eingangsberatung“ als eines der zentralen Handlungsfelder ausgewiesen. Wenngleich sich einhergehend mit schulstrukturellen Entwicklungen historisch recht unterschiedliche formale Professionalisierungsprozesse der verschiedenen Lehrämter nachzeichnen lassen (z. B. Terhart 2016, Kurtz 1997, Pleiß 1973), gibt es mittlerweile doch einen gewissen Konsens in der Bestimmung des professionellen Kerns dieses Berufs. So definiert die KMK $(2004 ; 2019)$ in den Standards für die Bildungswissenschaften die professionellen Anforderungen im Lehrberuf übergreifend als Unterrichten, Erziehen, Beurteilen und Beraten, sich selbst weiterbilden und Schule innovieren. Auch Modelle professioneller Kompetenz von Lehrpersonen differenzieren nicht zwischen einzelnen Lehrämtern, sondern weisen neben dem Bereich des professionellen Wissens, Überzeugungen und Werthaltungen, motivationale Orientierungen und selbstregulative Fähigkeiten als zentrale Kompetenzaspekte aus (z. B. Baumert \& Kunter 2006; Lehmann-Grube \& Nickolaus 2009). Motive, Überzeugungen und Werthaltungen gelten zudem übergreifend als relevante Einflussvariablen für die professionelle Entwicklung von Lehrpersonen (z. B. Cramer 2016).

Im öffentlichen Image des Lehrerberufs lassen sich ebenfalls übergreifende Attribute identifizieren, die nicht selten die Rahmenbedingungen der Tätigkeit adressieren und negativ konnotiert sind, wie z. B. „Lehrer als Halbtagsjobber“ (Schaarschmidt 2014) sowie in unzähligen Beamtenwitzen. Andererseits ist das Ansehen der einzelnen Lehrämter noch immer weitgehend an die Schulstrukturen gebunden. So genießt das gymnasiale Lehramt ein höheres Ansehen als alle anderen Lehrämter (Ebner \& Rohrbach-Schmidt 2019). Das öffentliche Bild gilt als Einflussfaktor für die Selbstelektion im Berufswahlprozess und 
könnte sich auch in Motiven, Überzeugungen und Werthaltungen niederschlagen, mit denen Lehramtsstudierende in den individuellen Professionalisierungsprozess einmünden. Selbstselektionsprozesse beim Zugang zum Lehramtsstudium sind daher sowohl für die Lehrkräftegewinnung, als auch für die Professionalisierung angehender Lehrpersonen relevant. Angesichts übergreifender Standards und Kompetenzmodelle stellt sich die Frage, ob und wie Gruppen mit ggf. unterschiedlichen bildungsbiografischen Voraussetzungen sich auch in ihren beruflichen Motivlagen und Überzeugungen unterscheiden? Oder führen Vorstellungen und Konzepte zum professionellen Kern des Lehrendenberufs dazu, dass trotz unterschiedlicher Zugangswege und Voraussetzungen beruflich weitgehend ähnlich motivierte und überzeugte Studierende anzutreffen sind, die auch im Hinblick auf KMK-Standards oder eines Kompetenzmodells mit ähnlichen Professionalisierungskonzepten adressiert werden können? Berufswahltheoretisch unter Bezugnahme auf den Person-Environment-Fit Ansatz lässt sich die Frage dahingehend verallgemeinern: Bis zu welchem Grad wirkt der Beruf trotz bildungsbiografischer Unterschiede homogenisierend in Bezug auf persönlichkeitsrelevante Voraussetzungen von Studienanfänger*innen im Lehramt?

Im Beitrag werden Studienvoraussetzungen von Lehramtsstudierenden in Bezug auf den Studienerfolg und die Studienzufriedenheit im ersten Studienjahr analysiert. Dies erfolgt unter einer vergleichenden Perspektive zwischen Studierenden im Lehramt an beruflichen Schulen $(\mathrm{LaB})$ und an Gymnasien $(\mathrm{LaG})$. Vergleiche von Studierenden der allgemeinen Lehrämter offenbaren Unterschiede in der sozialen Herkunft, dagegen nur geringe Unterschiede in der beruflichen Motivlage. Lehramtsstudierende der Sek I zeigen sich lediglich etwas stärker pädagogisch motiviert, während LaG-Studierende sich fachlich interessierter geben (Cramer 2016; Rothland 2014a, b; Ziegler 2009). Studierende des beruflichen Lehramts und des Lehramts an Gymnasien wurden bislang kaum miteinander verglichen. ${ }^{1}$ Da es sich um zwei laufbahnrechtlich gleichgestellte Lehrämter handelt und die Gleichstellung auch immer mit der Erwartung verbunden war, die Nachfrage im beruflichen Lehramt zu erhöhen, bietet sich der Vergleich zwischen Studienanfänger*innen im beruflichen Lehramt und im Lehramt an Gymnasien aber an. Insbesondere, weil über das Laufbahnrecht hinaus noch immer systemische, strukturelle und soziale Differenzen zwischen diesen beiden Lehrämtern bestehen.

Diese Differenzen variieren z. T. abhängig von kulturellen Traditionen in den Bundesländern. In Hessen, wo die hier analysierten Daten erhoben wurden, unterscheiden sich sowohl die Zulassungsbedingungen als auch der Abschluss trotz gesetzlich verbriefter Gleichstellung im Lehrerbildungsgesetz (HLbG). Das LaB-Studium ist konsekutiv aufgebaut und schließt mit dem

1 Bislang liegen diesbezüglich lediglich eine Studie von Driesel-Lange et al. (2017) und Ziegler und Berger (2019) sowie eine laufende Untersuchung an der TU Berlin (Micknass et al. 2019) vor. 
Master of Education ab, das LaG schließt nach wie vor mit dem Staatsexamen ab. Als Zugangsvoraussetzung im LaG wird noch immer das Abitur gefordert, während dagegen der Zugang zum LaB-Studium in der Bachelorphase lediglich einen Abschluss äquivalent zur Fachhochschulreife verlangt. Eine 52-wöchige Fachpraxis - den KMK-Vorgaben entsprechend - stellt im LaB eine höhere Zugangshürde dar, als im LaG der Nachweis eines vierwöchigen Orientierungspraktikums bis zum Antritt des ersten Schulpraktikums sowie ein achtwöchiges Betriebspraktikum bis zum ersten Staatsexamen. Vor dem Hintergrund einer bundesweiten und andauernden Rekrutierungsproblematik in den Studiengängen für das LaB gegenüber einer eher entspannten Versorgungssituation im $\mathrm{LaG}^{2}$, wirft dies Fragen nach Selektions- und Professionalisierungsprozessen auf, die auch über Hessen hinaus für die Professionalisierung angehender Lehrpersonen von Interesse sind.

\section{Methode}

Die im Beitrag präsentierten Befunde basieren auf einer Re-Analyse von Daten aus einer Evaluationsstudie zur Erfassung von Effekten einer neu konzipierten Studieneingangsphase für Studienfänger*innen im Lehramtsstudium an der TU Darmstadt. Die Neukonzeption erfolgte im Rahmen des Projekts MINTPLUS, gefördert über die Qualitätsoffensive Lehrerbildung. ${ }^{3}$ Das Theoriemodell der Evaluationsstudie wurde angelehnt an Blüthmann et al. (2008) entwickelt. In einem Panel sollten zwei Kohorten von Studienanfänger*innen, jeweils eine Kohorte vor und nach Implementation der neuen Beratungselemente, wie z. B. Peer-Beratung, Online-Self-Assessment, individuelle berufsbezogene Eignungsberatung etc., über das erste Studienjahr hinweg befragt werden. Es wurden individuelle Voraussetzungen und Kontextbedingungen sowie die Wahrnehmung der Studienbedingungen erhoben. Abhängige Variablen waren Studienzufriedenheit und Studienabbruchquoten. Die Studieneingangsphase gilt hier als besonders relevant (Heublein et al. 2017). Des Weiteren sollte der Zusammenhang zwischen individuellen Motiven und Überzeugungen und dem Informations- und Lernverhalten sowie die Entwicklung der Reflexionsbereitschaft untersucht werden. Nachdem jedoch einige der Beratungselemente schon der ersten Kohorte verfügbar gemacht wurden und auch die verbindliche

2 Im LaG gibt es allenfalls in einigen MINT-Fächern sowie in den Fächern Musik und Kunst Rekrutierungsprobleme, aber nicht so durchgängig und gravierend wie in den technischen Fächern des LaB.

3 BMBF FKZ: 01JA1514 
Nutzung der Beratungsangebote sich nicht umsetzen ließ, entsprechen die beiden Kohorten keiner definierten Kontroll- und Experimentalgruppe und somit nicht dem geplanten quasi-experimentellen Design. Für einen lehramtsspezifischen Vergleich bieten die Evaluationsdaten dennoch eine gewisse Ausgangsbasis.

\subsection{Stichprobe}

Zur Evaluation der Studieneingangsphase wurden seit dem Wintersemester 2016/ 2017 schriftlich-standardisierte Befragungen bei insgesamt zwei Kohorten (Grundgesamtheit, $n=363$ ) jeweils zu Studienbeginn (MZP 1) und zu Beginn des dritten Semesters (MZP 2) durchgeführt. An der Befragung zum MZP 1 nahmen $(n=209)$ Studierende teil, davon sind im Längsschnitt $(n=102)$ erfasst. Die Querschnittsstichprobe zum MZP 1 setzt sich aus $n=157$ LaGStudierenden und $\mathrm{n}=52 \mathrm{LaB}-\mathrm{Studierenden} \mathrm{zusammen.} \mathrm{Die} \mathrm{Querschnittsstich-}$ probe zum MZP 2 umfasst $n=119$ LaG-Studierende und $n=28$ LaB-Studierende. Im Längsschnitt konnten $20 \mathrm{LaB}$-Studierende und $82 \mathrm{LaG}$-Studierende erfasst werden. Die Verteilung von weiblichen und männlichen Studierenden zeigt sich im $\mathrm{LaG}$ mit $52 \% \mathrm{zu} 48 \%$ als ausgewogen. Im $\mathrm{LaB}$ verteilen sich weibliche und männliche Studierende im Verhältnis 60\% zu 35\%. 5,5\% machten keine Angaben. Zu Studienbeginn sind LaB-Studierende $(\mathrm{M}=24.26$; SD $=3.71)$ rund vier Jahre älter als $\mathrm{LaG}-$ Studierende $(\mathrm{M}=20.21 ; \mathrm{SD}=2.93)$. Weitere Angaben zu den Stichproben sind in Tabelle 1 abgetragen.

Tab. 1: Stichprobe

\begin{tabular}{|c|c|c|c|c|c|c|c|}
\hline & & \multicolumn{3}{|c|}{$\mathrm{LaG}$} & \multicolumn{3}{|c|}{$\mathrm{LaB}$} \\
\hline & & \multicolumn{2}{|c|}{ Geschlecht } & \multirow{2}{*}{$\frac{\text { Alter }}{M(S D)}$} & \multicolumn{2}{|c|}{ Geschlecht } & \multirow{2}{*}{$\frac{\text { Alter }}{M(S D)}$} \\
\hline & & w & $\mathrm{m}$ & & $\mathrm{w}$ & $\mathrm{m}$ & \\
\hline \multirow{3}{*}{ Querschnitt } & $\begin{array}{l}\text { MZP 1 } \\
(n=209)\end{array}$ & 73 & 83 & $\begin{array}{l}20.21 \\
(2.93)\end{array}$ & 22 & 28 & $\begin{array}{l}24.26 \\
(3.71)\end{array}$ \\
\hline & MZP 2 & & & 21.73 & & & 25.11 \\
\hline & $(n=147)$ & 52 & 51 & & 10 & 8 & \\
\hline & & & & 19.9 & & & 23.6 \\
\hline Längsschnitt & $(n=102)$ & 43 & 39 & $(2.18)$ & 7 & 12 & $(3.1)$ \\
\hline
\end{tabular}

Anm.: keine Angaben $n=30$

Quelle: eigene Darstellung 


\subsection{Instrumente}

In Tabelle 2 sind die verwendeten Instrumente mit der Itemanzahl und interner Konsistenz, gemessen mit Cronbachs Alpha, angegeben. Die Skalen zur Erfassung der Berufswahlmotivation sind weitgehend dem FEMOLA (Pohlmann \& Möller 2010) entnommen und wurden ergänzt durch Items aus dem Fit-Choice (Watt \& Richardson 2007). Die theoretische Grundlage des FEMOLA stellt das Erwartungs-Wert-Modell dar. Zu den Wertkomponenten zählt das Fachinteresse und die Nützlichkeit wie zum Beispiel in der Skala zur studienbezogenen Motivation. Die Skala der Fähigkeitsüberzeugung ist ein Teil der Erwartungskomponente, und die sozialen Einflüsse werden unter anderem durch die Skala zum sozialen Status abgebildet. Für die Messung der Reflexionsbereitschaft wurden Items eigens konstruiert und teilweise aus dem Instrument von Niggli (2004) übernommen. Die Reflexionsbereitschaft setzt sich aus folgenden drei Skalen zusammen (1) dem Interesse, das eigene Verhalten zu reflektieren (Interesse an Reflexion), (2) Überzeugungen zum Einfluss der Lehrerpersönlichkeit auf die Professionalität (Persönlichkeitsorientierung) und (3) der Bereitschaft, das Handeln durch Theorien belegen zu können (Theorienähe). Die Erwartung an die Fähigkeit, den Anforderungen auch in schwierigen Situationen gerecht werden zu können, wurde mit dem Instrument von Schmitz und Schwarzer (2002) zur Lehrerselbstwirksamkeitserwartung erfasst.

\section{Tab. 2: Verwendete Instrumente}

\begin{tabular}{|c|c|c|c|c|}
\hline \multirow[b]{2}{*}{ Fragebogen } & \multirow[b]{2}{*}{ Skala } & Beispielitem & \multirow[t]{2}{*}{ Items } & \multirow[t]{2}{*}{$\alpha$} \\
\hline & & $\begin{array}{l}\text { Ich habe das Lehramtsstudium ge- } \\
\text { wählt, weil ... }\end{array}$ & & \\
\hline \multirow{5}{*}{$\begin{array}{l}\text { Berufswahl- } \\
\text { motivation }\end{array}$} & $\begin{array}{l}\text { Umgang mit } \\
\text { jungen Menschen }\end{array}$ & $\begin{array}{l}\text {...ich gern mit jungen Menschen } \\
\text { arbeite. }\end{array}$ & 3 & .87 \\
\hline & $\begin{array}{l}\text { Vereinbarkeit Beru } \\
\& \text { Privatleben }\end{array}$ & $\begin{array}{l}\text { f...ich als Lehrer/in Familie und } \\
\text { Beruf gut vereinbaren kann. }\end{array}$ & 5 & .81 \\
\hline & $\begin{array}{l}\text { Studienbezogene } \\
\text { Motive }\end{array}$ & $\begin{array}{l}\text {...ich mich gern mit den Inhalten } \\
\text { meiner Fächer beschäftige. }\end{array}$ & 3 & .70 \\
\hline & Sozialer Status & $\begin{array}{l}\text {...Lehrer/innen einen hohen } \\
\text { sozialen Status haben. }\end{array}$ & 3 & .91 \\
\hline & $\begin{array}{l}\text { Fähigkeits- } \\
\text { überzeugung }\end{array}$ & $\begin{array}{l}\text {...mir schon häufiger rückgemeldet } \\
\text { wurde, Dinge verständlich erklären } \\
\text { zu können. }\end{array}$ & 5 & .75 \\
\hline
\end{tabular}




\begin{tabular}{|c|c|c|c|c|}
\hline & $\begin{array}{l}\text { Finanzielle } \\
\text { Sicherheit }\end{array}$ & ...ich als Lehrer/in gut verdiene. & 3 & .86 \\
\hline & $\begin{array}{l}\text { Intrinsisch } \\
\text { motiviert }\end{array}$ & $\begin{array}{l}\text {...ich viel in meinen Fächern dazu- } \\
\text { lernen möchte. }\end{array}$ & 15 & .82 \\
\hline \multirow{3}{*}{$\begin{array}{l}\text { Reflexions- } \\
\text { bereitschaft }\end{array}$} & $\begin{array}{l}\text { Persönlichkeits- } \\
\text { orientierung }\end{array}$ & $\begin{array}{l}\text { Um eine gute Lehrkraft zu } \\
\text { werden, benötigt man vor allem } \\
\text { Talent. }\end{array}$ & 4 & .68 \\
\hline & $\begin{array}{l}\text { Interesse an } \\
\text { Reflexion }\end{array}$ & $\begin{array}{l}\text { Nachüberlegungen zum Unterricht } \\
\text { sind genauso wichtig, wie die Un- } \\
\text { terrichtsplanung. }\end{array}$ & 11 & .65 \\
\hline & Theorienähe & $\begin{array}{l}\text { Ich finde, eine Lehrkraft sollte ihr } \\
\text { Handeln im Unterricht immer auch } \\
\text { theoretisch begründen können. }\end{array}$ & 9 & .83 \\
\hline $\begin{array}{l}\text { Studien- } \\
\text { interesse }\end{array}$ & & $\begin{array}{l}\text { Es war für mich von großer } \\
\text { persönlicher Bedeutung, gerade die- } \\
\text { sen Studiengang studieren zu kön- } \\
\text { nen. }\end{array}$ & 18 & .82 \\
\hline $\begin{array}{l}\text { Lehrerselbst- } \\
\text { wirksamkeit }\end{array}$ & & $\begin{array}{l}\text { Ich bin mir sicher, dass ich mich auf } \\
\text { individuelle Probleme der Schüler } \\
\text { gut einstellen können werde. }\end{array}$ & 7 & .67 \\
\hline
\end{tabular}

Anm.: $\alpha$ = Cronbachs Alpha. Die Skala ,Intrinsisch motiviert" setzt sich aus folgenden Skalen zusammen: Fähigkeitsüberzeugung, studienbezogene Motive, Umgang mit jungen Menschen, Erziehung und Interesse am Inhalt.

Quelle: eigene Darstellung

\section{Ergebnisse}

\subsection{Eingangsvoraussetzungen und Kontextbedingungen}

Ein Vergleich der Tätigkeit vor dem Studium (Abb. 1) zeigt, dass rund zwei Drittel der LaG-Studierenden unmittelbar vor Studienbeginn den Schulabschluss absolvierten oder zuvor in einem anderen Studiengang eingeschrieben waren. Die Gruppe der LaB-Studierenden absolvierte vor Studienbeginn zum größten Teil eine Berufsausbildung oder war berufstätig. Rund $50 \%$ der LaBStudierenden und 75\% der LaG-Studierenden gaben an, neben dem Studium einer Nebentätigkeit nachzugehen. 
Abb. 1: Tätigkeit vor dem Studium (längsschnittliche Daten)

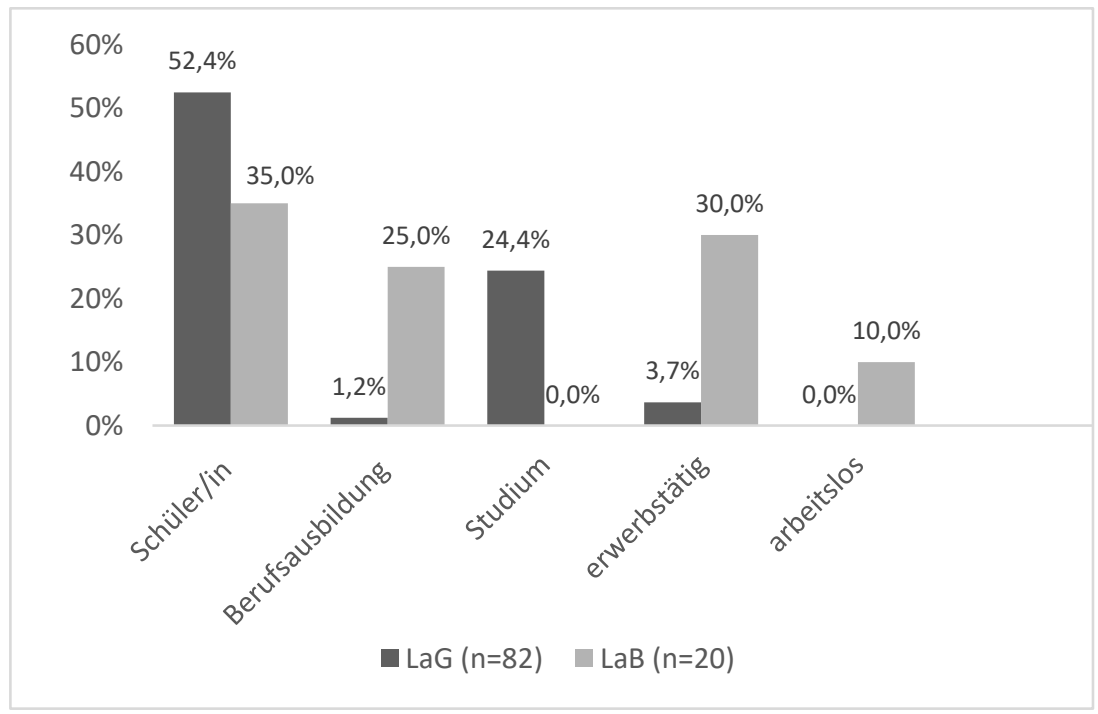

Quelle: eigene Darstellung

Die Ergebnisse lassen sich auch durch die Querschnittsdaten bestätigen (LaG $(n=154)$ : $50 \%$ Schüler*in, 26\% Studium, 10\%, 1,3\% Berufsausbildung erwerbstätig; LaB $(n=52)$ : $25 \%$ Schüler*in, $12 \%$ Studium, $19 \%$ Berufsausbildung, 33\% erwerbstätig). In Bezug auf die Hochschulzugangsberechtigung zeigen sich ebenfalls große Unterschiede zwischen den Gruppen. LaG-Studierende machten ihren Schulabschluss zum größten Teil auf einem Gymnasium oder Fachgymnasium (Längsschnitt: 87,6\%; Querschnitt: 94,8\%), während nur 36,8\% (Längsschnittstichprobe) bzw. 25\% (Querschnittsstichprobe) der LaBStudierende ihren Schulabschluss auf dem gymnasialen Zweig erlangt haben.

Die Note der Hochschulzugangsberechtigung unterscheidet sich hingegen nicht zwischen den Datenquellen (Längsschnitt: $t(100)=.869 ; p=.387$; Querschnitt: $t(172)=-1.335 ; p=.184)$.

Über Items zur Hochschulstudiumsmotivation sollte die explizite Motivation an einer Hochschule zu studieren, erfasst werden (Beispielitem: „Ich habe ein Hochschulstudium begonnen, weil ich mich gerne mit wissenschaftlichen Themen befasse."). Der Gruppenvergleich hinsichtlich der Motivation an einer Hochschule zu studieren, stellt sich im Längsschnitt nicht signifikant dar ( $t$ (99) $=1.84 ; p=.069)$, jedoch im Querschnitt $(t(207)=2.32 ; p=.021)$. Hinsichtlich der Studieninteressen zeigt sich ein signifikanter Unterschied in der Längsschnittstichprobe $(t(95)=2.961 ; p=.004)$, wie auch in der Querschnittstichprobe $(t(145)=3.08 ; p=.002)$, hier weisen LaB-Studierende ein geringeres 
Interesse auf. Die Fragen zum Studieninteresse fokussieren das studiengangbezogene Interesse (Beispielitem: ,Ich bin mir sicher, das Studium gewählt zu haben, welches meinen persönlichen Neigungen entspricht.“).

\subsection{Berufswahlmotivation}

Die Facetten der Berufswahlmotivation sind zwischen LaG- und LaB-Studierende größtenteils gleich ausgeprägt. Im Längsschnitt unterscheiden sich die Gruppen ausschließlich im Aspekt der finanziellen Sicherheit signifikant voneinander. Für LaB-Studierenden stellt die finanzielle Sicherheit ein stärkeres Motiv für die Berufswahl dar als für LaG-Studierende. Die Daten aus dem Querschnitt zeigen im Gegensatz zum Längsschnitt einen signifikanten Unterschied hinsichtlich der Motivation, sich mit den Inhalten des Lehramtstudiums zu beschäftigen (siehe Tab. 2 und Tab. 3 Skala: Studieren). Für die anderen Motive ergaben sich keine statistisch bedeutsamen Unterschiede zwischen den Gruppen. In Tabelle 3 sind die Ergebnisse im Überblick dargestellt.

Tab. 3: Berufswahlmotivation (Längsschnitt und Querschnitt MZP 1)

\begin{tabular}{lllllll}
\hline Längsschnitt & \multicolumn{2}{l}{ LaG } & \multicolumn{7}{l}{ LaB } & & \\
& $N$ & $M(S D)$ & $N$ & $M(S D)$ & $t(99)$ & $p$ \\
\cline { 2 - 7 } & 81 & $3.32(.42)$ & 20 & $3.3(.62)$ & .208 & .835 \\
Fähigkeitsüberzeugung & & & & & & \\
& 82 & $3.09(.66)$ & 20 & $3.04(.83)$ & .276 & .783 \\
$\begin{array}{l}\text { Vereinbarkeit Beruf \& } \\
\text { Privatleben }\end{array}$ & 81 & $3(.64)$ & 20 & $3.35(.62)$ & 2.196 & $.03 *$ \\
$\begin{array}{l}\text { Finanzielle } \\
\text { Sicherheit }\end{array}$ & 81 & $3.64(.47)$ & 20 & $3.62(.45)$ & .181 & .856 \\
$\begin{array}{l}\text { Umgang mit } \\
\text { Menschen }\end{array}$ & 81 & $3.7(.41)$ & 20 & $3.6(.45)$ & .987 & .331 \\
Studieren & & & & & & \\
& 81 & $2.19(.86)$ & 20 & $2.55(.89)$ & 1.668 & .099 \\
Sozialer Status & & & & & & \\
& 82 & $3.49(.31)$ & 20 & $3.41(.42)$ & .913 & .364 \\
$\begin{array}{l}\text { Intrinsische } \\
\text { Berufsmotivation }\end{array}$ & & & & & & \\
\hline
\end{tabular}




\begin{tabular}{lcccccc}
\hline Querschnitt & \multicolumn{2}{l}{$\mathrm{LaG}$} & \multicolumn{2}{c}{$\mathrm{LaB}$} & & \\
& $N$ & $M(S D)$ & $N$ & $M(S D)$ & $t(207)$ & $p$ \\
\cline { 2 - 7 } & 157 & $3.31(.46)$ & 52 & $3.28(.5)$ & .381 & .704 \\
$\begin{array}{l}\text { Fähigkeits- } \\
\text { überzeugung }\end{array}$ & & & & & & \\
$\begin{array}{l}\text { Vereinbarkeit Beruf } \\
\text { \& Privatleben }\end{array}$ & 157 & $3.06(.65)$ & 52 & $3.01(.72)$ & .428 & .669 \\
$\begin{array}{l}\text { Finanzielle } \\
\text { Sicherheit }\end{array}$ & 157 & $2.98(.64)$ & 52 & $3.03(.5)$ & .332 & .74 \\
$\begin{array}{l}\text { Umgang mit } \\
\text { Menschen }\end{array}$ & 157 & $3.61(.51)$ & 52 & $3.56(51)$ & .563 & .574 \\
$\begin{array}{l}\text { Studieren } \\
\text { Sozialer Status }\end{array}$ & 157 & $3.69(.42)$ & 52 & $3.53(.45)$ & 2.367 & $.019 *$ \\
& 157 & $2.15(.76)$ & 52 & $2.39(.59)$ & 1.935 & .054 \\
$\begin{array}{l}\text { Intrinsische } \\
\text { Berufsmotivation }\end{array}$ & 157 & $3.47(.32)$ & 52 & $3.43(.36)$ & .848 & .397 \\
\hline
\end{tabular}

Anm.: Die Skala reichte von 1 (niedrige Ausprägung) bis 5 (hohe Ausprägung).

Quelle: eigene Darstellung

\subsection{Reflexionsbereitschaft und Selbstwirksamkeitserwartung}

Bezogen auf die Reflexionsbereitschaft unterscheiden sich die Daten aus der Längsschnitt- und Querschnittuntersuchung. Werden die Daten aus der Längsschnittstichprobe betrachtet, zeigen LaG-Studierende im Vergleich zu den LaB-Studierenden eine höhere Bereitschaft zur Reflexion $(F(1,99)=7.24 ; p=$ $\left..008^{* *}\right)$. Ein weiterer bedeutsamer Unterschied bildet sich auf der Skala Persönlichkeitsorientierung ab. Auch hier ist die Ausprägung bei LaG-Studierenden höher $(F(1,98)=15.72 ; p<.000 * * *)$. Die Skala Theorienähe $(F(1,100)=$ $.172 ; p=.68)$ zeigt sich statistisch als nicht bedeutsam.

Die Daten aus dem Querschnitt weisen jedoch auf keine Gruppenunterschiede hinsichtlich der Faktoren Reflexionsinteresse $(t(145)=1.203 ; p=$ .231), Persönlichkeitsorientierung $(t(145)=1.275 ; p=.205)$ und Theorienähe $(t(145)=.975 ; p=.331)$ hin. 
Die Lehrer-Selbstwirksamkeitserwartung unterscheidet sich sowohl im Längsschnitt $(t(100)=1.01 ; p=.315)$ als auch im Querschnitt $(t(207)=1.026 ; p=$ .896) nicht signifikant zwischen LaG- und LaB-Studierenden.

\subsection{Informationsverhalten und Studienzufriedenheit}

Weiter wurde im Fragebogen zum ersten Messzeitpunkt die Informiertheit der Studierenden vor der Immatrikulation abgefragt. Die Informationsquellen lassen sich in die Kategorien Internet- und Printmedien, persönliche Beratung und persönliche Kontakte aufgliedern. Die Ergebnisse legen nahe, dass LaG-Studierende mehr Informationsquellen heranziehen als LaB-Studierende. Während dieser Vergleich längsschnittlich betrachtet nicht signifikant ist $(t(100)=$ $1.58, p=.118)$, zeigt der Querschnittsvergleich einen signifikanten Unterschied zwischen den beiden Gruppen $(t(205)=3.4 ; p=.001)$. Darüber hinaus sind signifikante Zusammenhänge zwischen der Informiertheit vor Studienbeginn mit der allgemeinen Studienzufriedenheit $\left(r=.208^{*}\right)$ sowie der Zufriedenheit mit dem ersten Fach $\left(r=.344^{*}\right)$ zu beobachten. Inwieweit sich die Studienzufriedenheit durch verschiedene Prädiktoren voraussagen lässt, wurde mit einer Regressionsanalyse überprüft. Ein gängiges Verfahren der multiplen linearen Regression überprüft schrittweise, welche Prädiktoren zur Vorhersage der allgemeinen Studienzufriedenheit geeignet sind, indem die signifikanteste Prädiktorvariable hinzugefügt und die am wenigsten signifikante Variable entfernt wird. Es zeigte sich ein Effekt $\left(\mathrm{R}^{2}=.257\right)$ mit den Prädiktoren erwartete Inhaltsbereiche im Studium $(\beta=.262 ; p=.002)$ und erwarteter Workload $(\beta=$ $.311 ; p<.000$ ). Die zum zweiten Messzeitpunkt abgefragten studienbezogenen Erwartungen bestätigen die Ergebnisse. LaG-Studierenden zeigen eine signifikant höhere allgemeine Zufriedenheit sowie eine höhere Zufriedenheit mit dem ersten Fach im Vergleich zu den LaB-Studierenden. Insgesamt werden die Erwartungen in sämtlichen Bereichen bei LaG-Studierenden deutlich besser erfüllt als bei LaB-Studierenden. Diese Ergebnisse werden durch die Daten der Querschnittserhebung bestätigt. Detaillierte Ausprägungen sind in Tabelle 4 abgetragen. 
Tab. 4: Inwiefern wurden die Erwartungen an das Studium erfüllt?

\begin{tabular}{|c|c|c|c|c|c|c|}
\hline \multirow[t]{2}{*}{ Längsschnitt } & \multicolumn{2}{|r|}{$\mathrm{LaG}$} & \multicolumn{2}{|r|}{$\mathrm{LaB}$} & \multirow[b]{2}{*}{$t(98)$} & \multirow[b]{2}{*}{$p$} \\
\hline & $N$ & $M(S D)$ & $N$ & $M(S D)$ & & \\
\hline Inhaltsbereiche 1. Fach & 81 & $5(1)$ & 18 & $3.3(1.41)$ & 4.569 & $.000 * * *$ \\
\hline Inhaltsbereiche Studium & 82 & $4.5(.99)$ & 19 & $3.84(1.3)$ & 2.531 & $.013 *$ \\
\hline Anforderungen 1. Fach & 81 & $4.65(1.28)$ & 17 & $3.65(1.32)$ & 2.941 & $.004 * *$ \\
\hline Workload & 82 & $4.37(1.25)$ & 19 & $3.15(1.61)$ & 3.583 & $.001 * *$ \\
\hline Vorkenntnisse 1. Fach & 81 & $4.41(1.43)$ & 18 & $3.55(1.72)$ & 2.201 & $.03 *$ \\
\hline Studienbedingungen & 81 & $4.4(1.15)$ & 19 & $4(1.37)$ & 1.3 & .197 \\
\hline Zufriedenheit Studium & 82 & $4.71(.96)$ & 19 & $3.58(1.07)$ & 4.51 & $.000^{* * *}$ \\
\hline Zufriedenheit 1. Fach & 80 & $4.85(1.16)$ & 17 & $3.53(1.42)$ & 4.096 & $.000 * * *$ \\
\hline \multirow[t]{2}{*}{ Querschnitt } & & $\mathrm{LaG}$ & & $\mathrm{LaB}$ & & \\
\hline & $N$ & $M(S D)$ & $N$ & $M(S D)$ & $t(140)$ & $p$ \\
\hline Inhaltsbereiche 1. Fach & 117 & $5.03(.99)$ & 26 & $3.62(1.55)$ & 5.91 & $.000^{* * *}$ \\
\hline Inhaltsbereiche Studium & 118 & $4.47(1.03)$ & 27 & $3.69(1.16)$ & 2.241 & $.027^{*}$ \\
\hline Anforderungen 1. Fach & 117 & $4.46(1.18)$ & 25 & $3.52(1.38)$ & 4.079 & $.000 * * *$ \\
\hline Workload & 118 & $4.2(1.22)$ & 27 & $3.19(1.5)$ & 3.738 & $.000 * * *$ \\
\hline Vorkenntnisse 1. Fach & 117 & $4.43(1.4)$ & 26 & $3.77(1.63)$ & 2.098 & $.038^{*}$ \\
\hline Studienbedingungen & 117 & $4.44(1.1)$ & 27 & $3.89(1.25)$ & 2.289 & $.024 *$ \\
\hline Zufriedenheit Studium & 118 & $4.69(1.1)$ & 27 & $3.67(1.11)$ & 4.69 & $.000^{* * *}$ \\
\hline Zufriedenheit 1. Fach & 115 & $4.94(1.11)$ & 25 & $3.84(1.55)$ & 4.159 & $.000 * * *$ \\
\hline
\end{tabular}

Anm.: Die Daten wurden zum zweiten Messzeitpunkt erhoben. Werte wurden auf einer Skala von 1 (niedrige Ausprägung) bis 6 (hohe Ausprägung) abgetragen.

Quelle: eigene Darstellung

Ein weiterer signifikanter Unterschied zeigt sich hinsichtlich der Credit Points. Die Gesamtzahl der erworbenen Credit Points in den ersten beiden Semestern fiel sowohl im Längsschnitt $(t(89)=2.44 ; p=.017)$ als auch im Querschnitt 
$(t(119)=2.592 ; p=.011)$ bei den LaG-Studierenden signifikant höher aus als bei den LaB-Studierenden.

\section{Diskussion}

Aus den Analysen sollten Hinweise bezüglich zweier Fragenperspektiven gewonnen werden. Zum einen die berufswahltheoretische Frage in Bezug auf den Person-Environment-Fit Ansatz, ob sich trotz bildungsbiografischer Unterschiede ähnliche Motivationsausprägungen und Überzeugungen bei den Studienanfänger*innen beider Lehrämter zeigen und dies dahingehend interpretiert werden kann, dass Berufe bzw. die verinnerlichten Berufskonzepte Personen mit ähnlichen Orientierungen anziehen und damit bezüglich der Selbstselektion eine homogenisierende Wirkung entfalten. Die zweite Fragenperspektive lässt sich als professionalisierungstheoretisch charakterisieren. Sie ist stärker auf den Zusammenhang zwischen biografischen und persönlichen Eingangsvariablen und Herausforderungen für die Professionalisierung von Lehramtsstudierenden gerichtet.

In Bezug auf die berufswahltheoretische Erkenntnisperspektive können die Ergebnisse als Bekräftigung der Homogenisierungsthese interpretiert werden, wonach Berufe Personen mit ähnlichen Überzeugungen und Motiven anziehen. Trotz erheblicher Unterschiede in den Bildungsbiografien unterscheiden sich beide Studierendengruppen nur geringfügig in ihrer Berufswahlmotivation und ihren berufsbezogenen Selbstwirksamkeitserwartungen. LaB-Studierende zeigen in Bezug auf den akademischen Kontext ein signifikant geringeres Studieninteresse, im Längsschnitt sind jene etwas stärker repräsentiert, die finanzielle Sicherheit als Berufswahlmotiv höher bewerten. Tendenziell ist auch der soziale Status für LaB-Studierende bedeutsamer als für LaG-Studierende. Studienanfänger*innen im LaG sind dagegen signifikant stärker von der Relevanz der Persönlichkeit im Lehrerberuf überzeugt und zeigen eine höhere Bereitschaft zur Reflexion. Beide Gruppen ähneln sich wiederum in ihrer Haltung zur theoriebasierten Begründungsverpflichtung im Lehrberuf (Theorienähe).

Im Hinblick auf die Professionalisierungsanforderungen sind die Ergebnisse ebenfalls aufschlussreich. Nach Kohli (1973) lässt sich die Motivlage der LaG-Studierenden auf den Polen zwischen einer direkten und indirekten Studienmotivation als etwas stärker direkt motiviert charakterisieren als die der LaB-Studierenden. Letztere begreifen das Studium noch mehr als Mittel zum Zweck auf dem Weg zum Beruf (indirekt motiviert), als dass sie das Studium 
auch zum Selbstzweck gewählt haben (direkt motiviert). Nachdem aber ansonsten die berufsbezogene Motivation recht ähnlich ist, könnten die Motivationsprofile auch als Indikatoren interpretiert werden, die vielmehr auf Effekte der Bildungssozialisation verweisen als auf Unterschiede in der Berufsmotivation. Auch die nachgewiesenen Differenzen im Informationsverhalten verweisen auf die Bildungssozialisation. LaB-Studierende nutzen weniger Informationsquellen und beginnen das Studium offensichtlich mit anderen Erwartungen als LaG-Studierende. Studienrealität und Erwartungen liegen bei LaB-Studierenden weiter auseinander und sie zeigen nach dem ersten Studienjahr eine signifikant geringere Zufriedenheit mit dem Studium als LaG-Studierende. Die geringere Studienzufriedenheit lässt sich regressionsanalytisch zu einem bedeutsamen Anteil auf die Inhalte der beruflichen Fachrichtung (1. Fach) und den geforderten Workload zurückführen. Studienanfänger*innen im LaB treffend ausgehend von ihren bisherigen Berufserfahrungen auf Anforderungen, die sie so nicht erwartet hatten und über die sie sich auch weniger informiert hatten, während LaG-Studierende eher darauf vorbereitet waren, weil sie sich ggf. das ,akademisch“ orientierte Lernen schon in der Schule aneigneten und auch unmittelbarer aus der Schule oder einem anderen Studium in das Lehramt einmünden. Hinzukommen könnte ein BFLP-Effekt ${ }^{9}$, wonach LaB-Studierende in ihrem bisherigen Kontext zu den erfolgreicheren Lerner*innen gehörten und nun auf eine neue Referenzgruppe treffen, in der sie diese Position verlieren. Dies systematisch zu erheben, musste aufgrund mangelnder Akzeptanz des Fragebogens leider aufgegeben werden. Es wäre jedoch eine relevante Fragestellung für künftige Untersuchungen. Die signifikanten Unterschiede in der Studienzufriedenheit sollten Anlass sein, für LaB-Studierende Brückenkonzepte zu entwickeln. Sie sollten zur Reflexion ihres beruflichen Übergangsprozesses angeleitet werden, um diesen produktiv zu bewältigen. Dazu ist geplant, Coaching oder Mentoringansätze zu erproben.

Nun handelt es sich hier, besonders im Längsschnitt, um eine recht kleine Stichprobe, was die Reichweite der Aussagen begrenzt. Zudem war die Untersuchung nicht von Anfang an auf die hier verfolgte Frageperspektive hin angelegt, sondern es handelt sich quasi um die Re-Analyse von Daten, die zur Evaluation einer neu gestalten Studieneingangsphase erhoben wurden. Dennoch offenbaren die Ergebnisse, dass vergleichende Forschung zum Lehrerberuf durchaus aufschlussreich sein kann und noch erheblicher Bedarf an weiteren Untersuchungen besteht.

9 „Big Fish Little Pond Effekt“ kann beim Wechsel in eine Bezugsgruppe mit anderem Leistungsniveau auftreten und sich z. B. negativ auf das akademische Selbstkonzept und die Lernmotivation niederschlagen, wenn das Leistungsvermögen der neuen Bezugsgruppe höher ist als das der vorausgehenden (z. B. Hannover \& Zander 2020). 


\section{Literatur}

Baumert, J. \& Kunter, M. (2006). Stichwort: Professionelle Kompetenz von Lehrkräften. Zeitschrift für Erziehungswissenschaft, 9(4), 469-520.

Blüthmann, I., Lepa, S. \& Thiel, F. (2008). Studienabbruch und -wechsel in den neuen Bachelorstudiengängen. Untersuchung und Analyse von Abbruchgründen. Zeitschrift für Erziehungswissenschaft, 11(3), 406-429.

Cramer, C. (2016). Personale Merkmale Lehramtsstudierender als Ausgangslage der professionellen Entwicklung. Dimensionen, Befunde und deren Implikationen für die Lehrerbildung. In A. Boeger (Hrsg.), Eignung für den Lehrerberuf (S. 31-56). Wiesbaden: Springer.

Driesel-Lange, K., Morgenstern, I. \& Keune, M. (2017). Wer wird Lehrer/in am Berufskolleg? Die Unterstützung von Professionalisierungsprozessen angehender Lehrpersonen für die Berufsbildung. In M. Becker, C. Dittmann, J. Gillen, S. Hiestand \& R. Meyer (Hrsg.), Einheit und Differenz in den gewerblich-technischen Wissenschaften. Berufspädagogik, Fachdidaktiken und Fachwissenschaften (S. 368-387). Münster: Lit. Verlag.

Ebener, C. \& Rohrbach-Schmidt, D. (2019) Berufliches Ansehen in Deutschland für die Klassifikation der Berufe 2010. Beschreibung der methodischen Vorgehensweise, erste deskriptive Ergebnisse und Güte der Messung. Version 1.0. Bonn: BIBB

Hannover, B. \& Zander, L. (2020). Wie personales und soziales Selbst die schulische Entwicklung von Kindern und Jugendlichen beeinflussen. Zeitschrift für Pädagogische Psychologie, 34, 65-85.

Heublein, U., Ebert, J., Hutzsch, C., Isleib, S., König, R., Richter, J. \& Woisch, A. (2017). Zwischen Studienerwartungen und Studienwirklichkeit. Ursachen des Studienabbruchs, beruflicher Verbleib der Studienabbrecherinnen und Studienabbrecher und Entwicklung der Studienabbruchquote an deutschen Hochschulen. Deutsches Zentrum für Hochschul- und Wissenschaftsforschung, Hannover.

Kohli, M. (1973). Studium und berufliche Laufbahn: über den Zusammenhang von Berufswahl und beruflicher Sozialisation. Stuttgart: Enke.

Kurtz, T. (1997). Professionalisierung im Kontext sozialer Systeme. Opladen: Westdeutscher Verlag.

Lehmann-Grube, S. \& Nickolaus, R. (2009). Professionalität als kognitive Disposition. In O. Zlatkin-Troitschanskaia, K. Beck, D. Sembill, R. Nickolaus \& R. Mulder (Hrsg.), Lehrprofessionalität. Bedingungen, Genese, Wirkungen und ihre Messung (S. 59-70). Weinheim: Beltz.

Micknass, A, Ohlemann, S., Pfetsch, J. \& Ittel, A. (2019). Berufswahlmotive von Studierenden des beruflichen Lehramts. In F. Gramlinger, C. Iller, A. Ostendorf, K. Schmid \& G. Tafner (Hrsg.). Bildung = Berufsbildung?! (S. 185-198). Bielefeld: 2019 wbv Publikation.

Niggli, A. (2004). Welche Komponenten reflexiver beruflicher Entwicklung interessieren angehende Lehrerinnen und Lehrer? - Faktorenstruktur eines Fragebogens und erste empirische Ergebnisse. Schweizerische Zeitschrift für Bildungswissenschaften, 26(2), 343-364.

Pleiß, U. (1973). Wirtschaftslehrerbildung und Wirtschaftspädagogik: die wirtschaftspädagogische Disziplinbildung an deutschsprachigen wissenschaftlichen Hochschulen. Göttingen: Schwarz. 
Pohlmann, B. \& Möller, J. (2010). Fragebogen zur Erfassung der Motivation für die Wahl des Lehramtsstudiums (FEMOLA). Zeitschrift für Pädagogische Psychologie, 24(1), 73-84.

Rothland, M. (2014a). Wer entscheidet sich für den Lehrerberuf? Herkunfts-, Persönlichkeits- und Leistungsmerkmalen von Lehramtsstudierenden. In E. Terhart, H. Bennewitz \& M. Rothland (Hrsg.), Handbuch der Forschung zum Lehrerberuf (S. 349-385). Münster: Waxmann.

Rothland, M. (2014b). Warum entscheiden sich Studierende für den Lehrerberuf? In E. Terhart, H. Bennewitz \& M. Rothland (Hrsg.), Handbuch der Forschung zum Lehrerberuf (2. überarb. u. erw. Aufl., S. 349-385). Münster: Waxmann.

Schaarschmidt, U. (2005). Halbtagsjobber? Psychische Gesundheit im Lehrerberuf Analyse eines veränderungsbedürftigen Zustandes. Weinheim u. a.: Beltz.

Schmitz, G. S., \& Schwarzer, R. (2002). Individuelle und kollektive Selbstwirksamkeitserwartung von Lehrern. Zeitschrift für Pädagogik, 44. Beiheft, 192-214.

Ständige Konferenz der Kultusminister (2014). Standards für die Lehrerbildung: Bildungswissenschaften. (Beschluss der Kultusministerkonferenz vom 16.12.2004 i. d. F. vom 16.05.2019). Online: https:/www.kmk.org/fileadmin/veroeffentlichungen_beschluesse/2004/2004_12_16-Standards-Lehrerbildung-Bildungswissenschaften.pdf (10.12.2019).

Terhart, E. (2016). Geschichte des Lehrerberufs In M. Rothland (Hrsg.), Beruf Lehrer/Lehrerin. Ein Studienbuch (S. 17-32). Münster: Waxmann.

Watt, H. M., \& Richardson, P. W. (2007). Motivational factors influencing teaching as a career choice: Development and validation of the FIT-Choice scale. The Journal of experimental education, 75(3), 167-202.

Ziegler, B. (2009). Genese von Professionalität - Berufsfindungs- und Berufswahlprozess. In O. Zlatkin-Troitschanskaia, K. Beck, D. Sembill, R. Nickolaus \& R. Mulder (Hrsg.), Lehrprofessionalität. Bedingungen, Genese, Wirkungen und ihre Messung (S. 413-423). Weinheim: Beltz.

Ziegler, B. (2019). Der Lehrerberuf als Gegenstand empirischer Berufsbildungsforschung. Editorial. Zeitschrift für Berufs- und Wirtschaftspädagogik, 115(4), 527540.

Ziegler, B. \& Berger, J. (2019). Kommen Lehrer an Gymnasien vom Mars und Lehrer an beruflichen Schulen vom Mond? Newsletter der Qualitätsoffensive Lehrerbildung des BMBF vom 29.5.2019. Online: https://www.qualitaetsoffensive-lehrerbildung.de/de/newsletter-der-qualitaetsoffensive-lehrerbildung-2-2019-2140. html (10.12.2019). 



\section{Die Qualitätsoffensive Lehrerbildung aus der Perspektive der beruflichen Lehrerbildung}

Silke Lange, Dietmar Frommberger, Ulrike Weyland und Eveline Wittmann

\section{Ausgangslage}

Die berufliche Lehrerbildung leidet im Vergleich zur allgemeinen Lehrerbildung unter einer geringeren Reputation, sowohl in ihrer gesellschaftlichen Wahrnehmung (Lipsmeier 2014) als auch in ihrer Position innerhalb der Universitäten und Pädagogischen Hochschulen. Zugleich sind die Aus- und Fort/Weiterbildungsbedarfe für diesen - mit sich stark verändernden Anforderungen konfrontierten - Lehramtstyp außerordentlich hoch (Klemm 2018; Lange, Frommberger 2020). Die Qualitätsoffensive Lehrerbildung, ein von Bund und Ländern initiiertes Förderprogramm zur Verbesserung der Lehrerbildung an Universitäten und Pädagogischen Hochschulen, trägt diesem Umstand mit der dritten Förderrunde gezielt Rechnung. Im Gegensatz zu den ersten beiden Förderrunden bildet die berufliche Lehrerbildung neben der Digitalisierung der Lehrerbildung einen Schwerpunkt der im November 2018 ausgeschriebenen dritten Förderrunde. Anträge zu dieser Förderrichtlinie konnten bis zum März 2019 eingereicht werden. Bereits im Mai 2019 wurden die vom Auswahlgremium zur Förderung empfohlenen Projekte in einer Pressemitteilung der Gemeinsamen Wissenschaftskonferenz (GWK) (2019) veröffentlicht.

Nach Bekanntgabe der geförderten Projekte wurde innerhalb der Sektion Berufs- und Wirtschaftspädagogik (BWP) der Deutschen Gesellschaft für Erziehungswissenschaft (DGfE) vor allem mit Blick auf den konkurrierenden Förderschwerpunkt zur Digitalisierung der Lehrerbildung diskutiert, inwieweit mit der Schwerpunktsetzung der dritten Förderrichtlinie zur Qualitätsoffensive Lehrerbildung sowie durch die zur Förderung empfohlenen Projektanträge die berufliche Lehrerbildung an den verschiedenen Standorten tatsächlich gefördert wird. Als Grundlage für weiterführende Diskussionen wurden Daten zur Antragstellung und zur Förderung der Projekte im Rahmen der dritten Förderrunde der Qualitätsoffensive ausgewertet und analysiert. Die Datenbasis bildeten veröffentliche Informationen zur Qualitätsoffensive Lehrerbildung und zu den als förderfähig bewerteten Projekten sowie Online-Umfragen des Vorstandes der Sektion BWP in der DGfE.

Mit diesem Beitrag werden die Analysen und deren Ergebnisse dargestellt. Im folgenden zweiten Abschnitt wird kurz das Programm der Qualitätsoffen- 
sive Lehrerbildung skizziert. Daran schließen sich die Analysen der Auswahlentscheidungen der ersten beiden Förderrunden (3. Abschnitt) und der dritten Förderrunde (4. Abschnitt) an. Im fünften Abschnitt werden die Ergebnisse der Umfragen des Vorstandes der Sektion BWP präsentiert. Auf diese Analysen aufbauend wird abschließend die Frage diskutiert, inwieweit es mit der dritten Förderrunde der Qualitätsoffensive Lehrerbildung gelingen kann, substanzielle Akzente für die berufliche Lehrerbildung zu setzen.

\section{Zum Programm der Qualitätsoffensive Lehrerbildung}

Die Qualitätsoffensive Lehrerbildung ist ein gemeinsam von Bund und Ländern getragenes Förderprogramm zur Weiterentwicklung der Lehrerbildung an Hochschulen in Deutschland. Ziel des wettbewerblich angelegten Verfahrens ist die Steigerung der Qualität und Attraktivität der Lehrerbildung, indem die Lehrerbildung an den Hochschulen profiliert, die Vergleichbarkeit der lehramtsbezogenen Studienleistungen und -abschlüsse verbessert und die Mobilität von Studierenden und Lehrkräften gewährleistet wird (Brümmer et al. 2018; GWK 2013). Dafür stellt das Bundesministerium für Bildung und Forschung (BMBF) auf Grundlage der in der GWK beschlossenen Bund-Länder-Vereinbarung (GWK 2013) bis 2023 bis zu 500 Millionen Euro zur Verfügung.

Die Entwicklungsimpulse setzen bereits im Prozess der Antragserarbeitung und Antragsstellung an. Die Einbindung der geplanten Projektaktivitäten in ein auf die standortspezifischen Stärken und Schwächen aufgebautes hochschulstrategisches Konzept zur Lehrerbildung soll ,die Sichtbarkeit und Wertschätzung der Lehrerbildung “ innerhalb der Hochschule fördern und die Relevanz der Projektaktivitäten gewährleisten (Brümmer et al. 2018). Dabei sollen Impulse gesetzt werden zur (A) Profilierung und Optimierung der Strukturen der Lehrerbildung, (B) Qualitätsverbesserung des Praxisbezugs, (C) Verbesserung der professionsbezogenen Beratung und Begleitung der Studierenden, (D) Stärkung der Querschnittsthemen Inklusion und Heterogenität in der Lehrerbildung, (E) Weiterentwicklung der Fachlichkeit, Didaktik und Bildungswissenschaften an den Hochschulen und (F) Verbesserung der Mobilität (BMBF 2014). Mit diesen Handlungsfeldern wird Bezug genommen auf die bereits Ende der 1990er Jahre von der Gemischten Kommission Lehrerbildung (Terhart 2000) identifizierten Defizite und Anforderungen der Lehrerbildung in Deutschland (auch Brümmer et al. 2018). Die Lehrerbildung für die beruflichen Schulen wird in der Ausschreibung zur dritten Förderrunde des Programms mit dem Handlungsfeld „Entwicklung neuer Ansätze für die Gewinnung und Qualifizierung von Lehrkräften an beruflichen Schulen“ explizit angesprochen. In weiteren Handlungsfeldern werden dagegen allge- 
meine Problembereiche der Lehrerbildung adressiert, ohne weitere Problembereiche der beruflichen Lehrerbildung zu berücksichtigen, z. B. werden die Polyvalenzthematik, die Wirklichkeit des Berufslebens, Verknüpfungen mit der Betriebspraxis oder berufsdidaktische Themen nicht systematisch aufgegriffen (BMBF 2014).

Zur Umsetzung der Qualitätsoffensive Lehrerbildung wurden in einem wissenschaftsgeleiteten Wettbewerbsverfahren in drei Förderrunden von einem 18-köpfigen Auswahlgremium, bestehend aus Vertreter*innen der Wissenschaft, der Studierendenschaft, der schulpraktischen Lernorte sowie von Bund und Ländern, Projekte zur Förderung ausgewählt, die verschiedene Lösungsansätze präsentieren und vor dem Hintergrund der standortspezifischen Situation der Lehrerbildung umsetzen. Dabei weisen die Standorte eine strukturelle Vielfalt bezogen auf u.a. ihre Größe, den Anteil der Lehramtsstudierenden, die angebotenen Lehramtsstudiengänge und die in die Projekte der Qualitätsoffensive Lehrerbildung einbezogenen Lehramtstypen auf. Der Zwischenbericht zur Programmevaluation (Brümmer et al. 2018) zeichnet bereits während der zweiten Förderrunde vielfältige Projektaktivitäten in den unterschiedlichen Handlungsfeldern der Qualitätsoffensive Lehrerbildung nach und konstatiert, dass das Programm ,,in den jeweiligen Handlungsfeldern bereits zu Veränderungen beigetragen" habe (ebd., 8). Gleichwohl kommen die Autor*innen zu dem Schluss, dass einige aktuelle Herausforderungen der Lehrerbildung mit dem Programm bis dahin nicht oder unzureichend adressiert wurden. Dazu zählen die Digitalisierung in der Lehrerbildung, die berufliche Lehrerbildung, welche mit ihren spezifischen Herausforderungen in die Projekte der ersten beiden Förderrunden an den Standorten demnach oft zu wenig involviert war, der Lehrkräftemangel und die Internationalisierung der Lehrerbildung. Auf diese Kritik bezugnehmend wurde eine dritte Förderrunde der Qualitätsoffensive Lehrerbildung mit den Schwerpunkten Digitalisierung und Lehrerbildung für die beruflichen Schulen initiiert.

\section{Die berufliche Lehrerbildung in der ersten und zweiten Förderrunde der Qualitätsoffensive Lehrerbildung}

In der ersten Förderrunde (2015-2018 bzw. 2016-2019) der Qualitätsoffensive Lehrerbildung wurden aus 133 eingereichten Antragskonzepten 49 Projekte an 59 Standorten zur Förderung ausgewählt (DLR o. J.). Die Auswahl der zur Förderung empfohlenen Projekte erfolgte anhand der in der Richtlinie beschriebenen Auswahlkriterien. Eine fachliche Vorbegutachtung der Anträge erfolgte durch fachnahe Gutachter*innen, für die die „Befangenheitsregelungen der DFG umfassend berücksichtigt" (Drucksache 19/11886, 7) wurden. 
Unter den geförderten Projekten adressier(t)en nur die Projekte der Universität Stuttgart (LEBUS) und der TU Berlin (TUB Teaching) sowie das Verbundprojekt der Universitäten Hannover und Osnabrück (PLanC) explizit das berufliche Lehramt. In 29 weiteren Projekten wird das berufliche Lehramt neben anderen Lehramtstypen adressiert. Auswertungen der Projektevaluation (Brümmer et al. 2018, 23) haben ergeben, dass das berufliche Lehramt in rund 59 Prozent der geförderten Projekte der ersten Förderphase adressiert und damit häufiger einbezogen wurde als die Lehramtstypen I (Grundschule bzw. Primarstufe, 54\%), VI (Sonderpädagogik, 36\%) und II (Primarstufe und Sekundarstufe I), aber seltener als die Lehramtstypen III (Sekundarstufe I, 73\%) und IV (Gymnasium, 86\%). Über den Umfang der Einbindung gibt die Zwischenevaluation keine Auskunft. Wie stark die einzelnen Lehramtstypen an den Standorten tatsächlich eingebunden werden, lässt sich bisher nicht weiter beschreiben.

Mit der zweiten Förderphase, die sich nur an bereits geförderte Projekte richtete, konnten 48 der 49 Projekte der ersten Förderphase ihre Projektaktivitäten fortführen (BMBF o. J. a, b). Nicht weiter gefördert wurde ein Verbundprojekt, das auf die Gewinnung und Qualifizierung potentieller Studienumsteiger*innen für das berufliche Lehramtsstudium abzielte (BMBF o. J. c). Zu vermuten ist, dass die Weiterförderung möglicherweise daran scheiterte, dass die Zahl der im Rahmen des Projektkontextes ,umgestiegenen“ Personen verhältnismäßig klein ausgefallen sei und damit „das Reservoire der potentiellen ingenieurwissenschaftlichen Studienumsteiger*innen den Bedarf des Lehrkräftemangels in den Mangelfachrichtungen der Elektro- und Metalltechnik langfristig nicht decken“ könne. Insofern, so die Projektbeteiligten, ,wird auch zukünftig die Aufgabe bestehen bleiben, weitere Personengruppen aufzudecken und in den Fokus der Rekrutierung zu nehmen“"(Gillen et al. 2018, 36 f.).

In der zweiten Förderphase adressiert nur noch das Projekt der Universität Stuttgart ausschließlich das berufliche Lehramt. Mit dem Projekt „TUB Teaching 2.0" wird an der TU Berlin in der zweiten Förderphase zusätzlich das Lehramt an Gymnasien einbezogen (BMBF o. J. d). Darüber hinaus beziehen sich die Projektaktivitäten 26 weiterer Projekte u.a. auch auf das berufliche Lehramt. Damit ist insgesamt die Zahl der Projekte, die das berufliche Lehramt fokussieren oder einbeziehen, im Gegensatz zur ersten Förderphase leicht gesunken auf insgesamt 27 Projekte. Nach eigenen Berechnungen ist die Quote für das berufliche Lehramt damit leicht auf rund 56 Prozent gesunken.

Insgesamt wurden in der zweiten Förderrunde rund 197,7 Millionen Euro bewilligt. Auf die 27 Projekte, die das berufliche Lehramt berücksichtigen, entfallen insgesamt rund 115,7 Millionen Euro (59\% des Gesamtfördervolumens der zweiten Förderphase), wobei zu berücksichtigen ist, dass dieses anteilige Fördervolumen in den Projekten in der Mehrzahl der Projekte auf zwei oder mehr Lehrämter aufgeteilt wurde. Angenommen, alle im Projekt adressierten Lehrämter würden von der Förderung gleichermaßen berücksichtigt, 
ergäbe sich eine rechnerische Fördersumme für das berufliche Lehramt in der zweiten Förderphase von rund 31,1 Millionen Euro (16\% des Gesamtfördervolumens in der zweiten Förderphase). Etwa die Hälfte der Projekte der Qualitätsoffensive Lehrerbildung berücksichtigt in seinen Projektaktivitäten das berufliche Lehramt, jedoch ist zumindest anzunehmen (unter der oben getroffenen Annahme), dass deutlich weniger Fördermittel für einen Innovationsschub im beruflichen Lehramt zur Verfügung gestellt werden. Dafür spricht auch, dass die Projektevaluation im Zwischenbericht zu dem Schluss kommt, dass ,,an den QLB-Hochschulen [...] berufliches Lehramt häufig studiert werden“ könne, dieses ,,aber in die QLB-Projekte an den Standorten [...] oft wenig involviert" sei (ebd., 31). Auch die Auswahlkriterien seien für das berufliche Lehramt „nicht ganz passend“ (ebd., 34). Insgesamt hätten die Projekte, die das berufliche Lehramt adressieren, ein „Wahrnehmungsproblem“ innerhalb der Hochschulen und innerhalb des Programms (ebd., 126). Daher bedürfe es einer ,stärkere[n] Aufmerksamkeit für das berufliche Lehramt mit seinen z. T. besonderen Anforderungen und entsprechenden bildungswissenschaftlichen und fachdidaktischen Modellen“" (ebd., 31).

\section{Analyse der Ergebnisse der Auswahlentscheidung zur dritten Förderphase}

Mit der dritten Förderphase (2020-2023) der Qualitätsoffensive Lehrerbildung, an der sich wieder alle lehrerbildenden Universitäten und Pädagogischen Hochschulen beteiligen konnten, wurde die berufliche Lehrerbildung stärker fokussiert. Neben einem Schwerpunkt „Digitalisierung in der Lehrerbildung“ wurde ein Schwerpunkt „Lehrerbildung für die beruflichen Schulen“ ausgeschrieben. Aufgrund einer Kleinen Anfrage von FDP-Abgeordneten im Bundestag (Drucksache 19/11886) liegen detaillierte Informationen zu den eingereichten und den zur Förderung empfohlenen Projekten der dritten Förderphase vor, die im Folgenden analysiert werden.

Insgesamt wurden 66 Projektskizzen zur dritten Förderrichtlinie der Qualitätsoffensive Lehrerbildung eingereicht, davon 18 Skizzen im Schwerpunkt berufliche Lehrerbildung (15 Einzel- und 3 Verbundvorhaben), 40 Skizzen im Schwerpunkt Digitalisierung (34 Einzel- und 6 Verbundvorhaben) und acht Skizzen zu beiden Schwerpunkten (7 Einzel- und 1 Verbundvorhaben). Von den eingereichten Projektskizzen wurden 43 Skizzen von dem eingesetzten Auswahlgremium zur Förderung empfohlen. Davon sind 13 Skizzen dem Schwerpunkt berufliche Lehrerbildung ( $72 \%$ der im Schwerpunkt beantragten Projekte), 26 Skizzen dem Schwerpunkt Digitalisierung (65\% der im Schwerpunkt beantragten Projekte) und vier Skizzen beiden Schwerpunkten (50\% der in den beiden Schwerpunkten beantragten Projekte) zugeordnet. 
Mit der dargestellten Auswahlentscheidung entfallen rund 30 Prozent der zur Förderung empfohlenen Projekte auf den Schwerpunkt berufliche Lehrerbildung und rund neun Prozent der zur Förderung beantragten Projekte sind beiden Schwerpunkten zuzuordnen. Keine Angaben lassen die bisher veröffentlichten Informationen darüber zu, wie hoch der Anteil der Projekte insgesamt ist, in denen die berufliche Lehrerbildung adressiert wird. Es ist zumindest davon auszugehen, dass unter den dem Schwerpunkt Digitalisierung zugeordneten Projekten einige auch das berufliche Lehramt adressieren, allerdings nur unter dem Gesichtspunkt der Digitalisierung. Dementsprechend ist zwar anzunehmen, dass deutlich mehr als die 17 Projekte, die dem Schwerpunkt berufliche Lehrerbildung oder beiden Schwerpunkten zugeordnet sind, das berufliche Lehramt adressieren und damit die Quote der Projekte, in denen die berufliche Lehrerbildung mindestens einbezogen ist, erheblich über 39 Prozent liegen wird. Doch kann aufgrund der fehlenden Daten bisher nicht eingeschätzt werden, ob mit der Fokussierung der beruflichen Lehrerbildung als ein Schwerpunkt der Ausschreibung insgesamt der Anteil der Projekte gestiegen ist, die das berufliche Lehramt adressieren, und inwiefern die qualitativen Entwicklungsbedarfe der beruflichen Lehrerbildung adressiert werden. Unter der Annahme, dass die im Schwerpunkt berufliche Lehrerbildung eingereichten und zur Förderung empfohlenen Projekte ausschließlich das berufliche Lehramt adressieren, ist jedoch zu konstatieren, dass der Anteil der Projekte, die ausschließlich das berufliche Lehramt fokussieren, im Vergleich zu den ersten beiden Förderrunden deutlich gestiegen ist (1. Förderrunde: $6 \% ; 2$. Förderrunde: $2 \%$; 3. Förderrunde: mind. $30 \%{ }^{1}$ ). Insofern ist es mit der Schwerpunktsetzung der Ausschreibungsrichtlinie der dritten Förderphase durchaus gelungen, dass deutlich mehr Universitäten und Pädagogische Hochschulen einen Einzelantrag (von einem einzelnen Standort) und/oder einen Verbundantrag mit einem anderen Standort oder mehreren anderen Standorten gemeinsam mit Fokus auf die berufliche Lehrerbildung auf den Weg gebracht haben. Die Sichtbarkeit der beruflichen Lehrerbildung an den Hochschulstandorten scheint damit gestiegen zu sein.

1 Da nicht abzuschätzen ist, wie viele Projekte, die dem Schwerpunkt Digitalisierung oder beiden Schwerpunkten zugeordnet sind, auch nur das berufliche Lehramt fokussieren, ist davon auszugehen, dass die Quote der Projekte, die ausschließlich das berufliche Lehramt fokussieren, möglicherweise noch höher ist. 


\section{Beantragtes Fördervolumen: $133.964 .531 €$}

\section{Zur Förderung empfohlenes \\ Gesamtvolumen: $77.202 .550 €$}

...davon im Schwerpunkt Digitalisierung:

$50.511 .857 €$

...davon im Schwerpunkt Berufliche Bildung:

$19.893 .560 €$

...davon in Schwerpunkt beiden Schwerpunkten:

$6.797 .133 €$

Quelle: eigene Darstellung, Daten entnommen aus Drucksache 19/11886

Mehr als 133,9 Millionen Euro wurden im Rahmen der dritten Förderrunde beantragt, zur Förderung empfohlen wurden Projekte mit einem Gesamtfördervolumen von rund 77,2 Millionen Euro (58\% des beantragten Fördervolumens). Mit einem Anteil von rund 26 Prozent des empfohlenen Gesamtfördervolumens (rund 19,9 Mio. €, vgl. Abb. 1) liegt der Anteil des empfohlenen Fördervolumens im Schwerpunkt berufliche Lehrerbildung etwas unter der Quote der zur Förderung empfohlenen Projekte in diesem Schwerpunkt (30\% der zur Förderung empfohlenen Projekte). Das spiegelt sich auch in der ermittelten durchschnittlichen Projektfördersumme wider. Diese ermittelte durchschnittliche Projektfördersumme ${ }^{2}$ beschreibt, welche Fördersumme für ein Projekt in der dritten Förderrunde durchschnittlich zur Förderung empfohlen wurde. Ermittelt wurden (Abb. 2) eine durchschnittliche Projektfördersumme auf Basis der Gesamtfördersumme und der Gesamtzahl der Projekte (Gesamt) sowie durchschnittliche Projektfördersummen in Bezug auf die ausgeschriebenen Schwerpunkte als Quotient der für den Schwerpunkt empfohlenen Gesamtfördersumme und der Zahl der zur Förderung im Schwerpunkt empfohlenen Projekte. Diese unterschiedlichen ermittelten durchschnittlichen Projektfördersummen sind in Abbildung 2 veranschaulicht. Wie die Abbildung 2 zeigt, ist die ermittelte durchschnittliche Projektfördersumme im Schwerpunkt

2 Die ermittelte durchschnittliche Projektfördersumme (nach Förderschwerpunkt) wurde berechnet als Quotient aus dem Gesamtfördervolumen je Förderschwerpunkt und der Anzahl der zur Förderung empfohlenen Projekte des Förderschwerpunktes. 
Digitalisierung am höchsten. Sie liegt mit rund 1,9 Millionen Euro je Projekt rund 400.000 Euro oberhalb der durchschnittlichen Projektfördersumme der Projekte im Schwerpunkt berufliche Lehrerbildung, die mit rund 1,5 Millionen Euro je Projekt zur Förderung empfohlen wurden, und rund 200.000 Euro oberhalb der durchschnittlichen Projektfördersumme insgesamt (vgl. Abb. 2). Das bedeutet: Ein Projekt im Schwerpunkt berufliche Lehrerbildung erhält im Durchschnitt weniger Fördermittel als ein Projekt im Schwerpunkt Digitalisierung.

Abb. 2: Ermittelte durchschnittliche Fördersumme für die zur Förderung empfohlenen Projekte nach Schwerpunkten

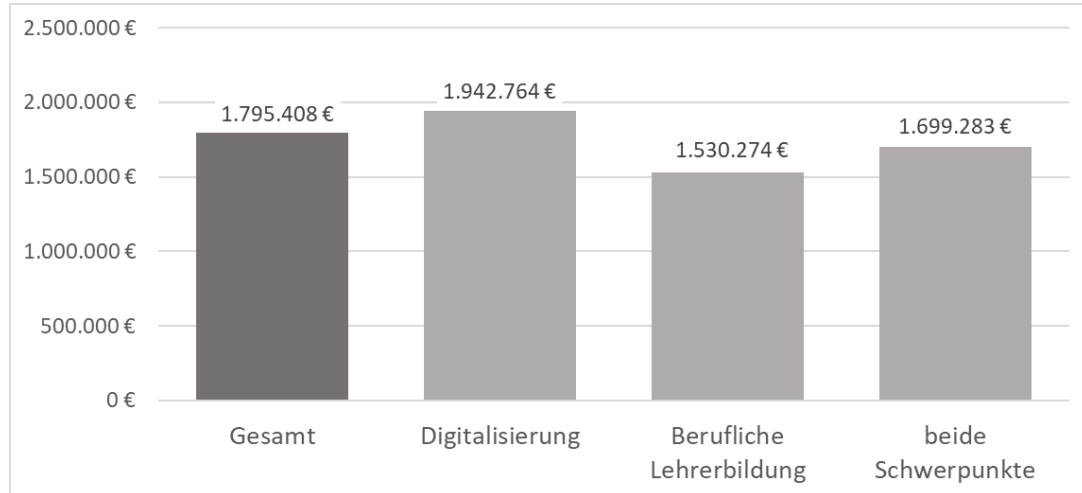

Quelle: eigene Darstellung, Daten entnommen aus Drucksache 19/11886

Ein Blick in die Bundesländer zeigt ein differenziertes Bild. Innerhalb der elf Bundesländer, die eine Förderung im Schwerpunkt berufliche Lehrerbildung oder in beiden Schwerpunkten erhalten, werden jeweils zwischen einem (Mecklenburg-Vorpommern, Rheinland-Pfalz, Sachsen und Schleswig-Holstein) und maximal drei Projekten (Niedersachsen) gefördert (vgl. Abb. 3, links). Projekte, die beiden Schwerpunkten zugeordnet werden, wurden in der Darstellung in Abbildung 3 und in der Analyse der Daten zur Hälfte der beruflichen Lehrerbildung zugerechnet, da keine Informationen darüber vorliegen, wie hoch der Anteil der beruflichen Lehrerbildung in den beantragten Projekten tatsächlich ist. Länderübergreifende Verbundprojekte wurden zu gleichen Anteilen den Projektpartnern zugewiesen.

Das Gesamtfördervolumen der zur Förderung empfohlenen Projekte im Schwerpunkt berufliche Lehrerbildung verteilt sich ebenfalls ungleichmäßig auf die Bundesländer (vgl. Abb. 3, rechts). Ebenso wie bei der Analyse der Projekte wurde auch bei der Analyse der Fördersummen das Fördervolumen von Anträgen, die beiden Schwerpunkten zugeordnet werden können, für die 
berufliche Lehrerbildung nur hälftig berücksichtigt. Fördersummen für Verbundprojekte wurden gleichmäßig auf die Verbundpartner aufgeteilt.

\section{Abb. 3: Anzahl ${ }^{3}$ (links) und Fördervolumen (rechts) der zur Förderung empfohlenen Anträge, die dem Schwerpunkt berufliche Lehrerbildung oder beiden Schwerpunkten zugeordnet sind, nach Bundesländern}
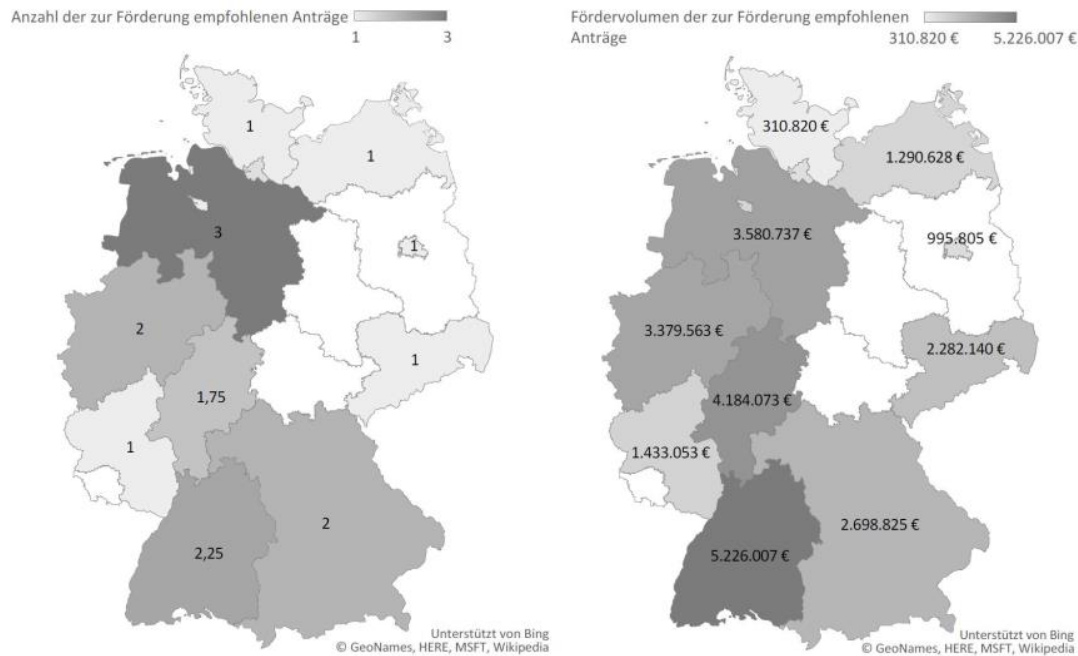

Quelle: eigene Darstellung, Daten entnommen aus Drucksache 19/11886

Mit rund 5,2 Millionen Euro konnte Baden-Württemberg für 2,25 Projekte die höchste Fördersumme für die berufliche Lehrerbildung einwerben, mit etwas Abstand folgt Hessen mit rund 4,2 Millionen Euro für 1,75 Projekte. Das geringste Fördervolumen wird mit rund 311.000 Euro für Schleswig-Holstein (1 Projekt) ausgewiesen. Durchschnittlich konnten vor allem Hessen (rund 2,4 Mio. $€$ je Antrag), Baden-Württemberg und Sachsen (jeweils rund 2,3 Mio. $€$ je Antrag) große Projekte zur beruflichen Lehrerbildung platzieren. Mit Blick auf die Zahl der Studienstandorte und die Studierendenzahlen im beruflichen Lehramt (Frommberger \& Lange 2018) erscheint diese Verteilung durchaus plausibel, wenngleich einige große Standorte der beruflichen Lehrerbildung keine Förderung im Rahmen der dritten Förderrunde der Qualitätsoffensive Lehrerbildung erhalten ${ }^{4}$.

3 Die Nachkommastellen kommen durch die Aufteilung der Projekte zu beiden Schwerpunkten auf die einzelnen Schwerpunkte und / oder die Aufteilung eines Verbundprojektes auf die Projektpartner zustande.

4 Inwiefern von diesen Standorten Anträge vorlagen, kann aufgrund der veröffentlichten, offiziellen Datenlage nicht erschlossen werden. 
Die scheinbar ungleichmäßige Verteilung der Mittel zwischen den Bundesländern, die sich auch für das gesamte Fördervolumen der dritten Förderphase zeigt, lenkt den Blick auf die Verteilungsmechanismen zwischen den Bundesländern.

Tab. 1: Verteilung des Fördervolumens gem. Königssteiner Schlüssel

\begin{tabular}{|c|c|c|c|c|}
\hline Bundesland & $\begin{array}{c}\text { empfohlenes } \\
\text { Förder- } \\
\text { volumen }\end{array}$ & $\begin{array}{l}\text { Förderanteil } \\
\text { gem. } \\
\text { Königssteiner } \\
\text { Schlüssel }\end{array}$ & $\begin{array}{l}\text { Differenz der } \\
\text { Fördersumme } \\
\text { zum } \\
\text { Königssteiner } \\
\text { Schlüssel }\end{array}$ & $\begin{array}{l}\text { relative } \\
\text { Differenz }\end{array}$ \\
\hline $\begin{array}{l}\text { Baden- } \\
\text { Württemberg }\end{array}$ & $15.636 .569 €$ & $10.046 .213 €$ & $+5.590 .355 €$ & $+55,65 \%$ \\
\hline Bayern & $7.510 .103 €$ & $12.016 .507 €$ & $-4.506 .404 €$ & $-37,50 \%$ \\
\hline Berlin & $995.805 €$ & $3.966 .312 €$ & $-2.970 .507 €$ & $-74,89 \%$ \\
\hline Bremen & & $2.329 .988 €$ & $-2.329 .988 €$ & $-100,00 \%$ \\
\hline Hamburg & $1.309 .043 €$ & $743.337 €$ & $+565.706 €$ & $+76,10 \%$ \\
\hline Hessen & & $1.974 .764 €$ & $-1.974 .764 €$ & $-100,00 \%$ \\
\hline $\begin{array}{l}\text { Mecklenburg- } \\
\text { Vorpommern }\end{array}$ & $7.108 .898 €$ & $5.746 .525 €$ & $+1.362 .372 €$ & $+23,71 \%$ \\
\hline Niedersachsen & $1.290 .628 €$ & $1.531 .845 €$ & $-241.217 €$ & $-15,75 \%$ \\
\hline $\begin{array}{l}\text { Nordrhein- } \\
\text { Westfalen }\end{array}$ & $11.190 .506 €$ & $7.264 .706 €$ & $+3.925 .800 €$ & $+54,04 \%$ \\
\hline $\begin{array}{l}\text { Rheinland- } \\
\text { Pfalz }\end{array}$ & $15.320 .258 €$ & $16.279 .516 €$ & $-959.258 €$ & $-5,89 \%$ \\
\hline Saarland & $3.888 .557 €$ & $3.724 .707 €$ & $+163.850 €$ & $+4,40 \%$ \\
\hline Sachsen & $899.291 €$ & $927.951 €$ & $-28.660 €$ & $-3,09 \%$ \\
\hline $\begin{array}{l}\text { Sachsen- } \\
\text { Anhalt }\end{array}$ & $7.538 .814 €$ & $3.853 .063 €$ & $+3.685 .751 €$ & $+95,66 \%$ \\
\hline $\begin{array}{l}\text { Schleswig- } \\
\text { Holstein }\end{array}$ & $2.496 .778 €$ & $2.124 .336 €$ & $+372.442 €$ & $+17,53 \%$ \\
\hline Thüringen & $1.157 .756 €$ & $2.628 .948 €$ & $-1.471 .192 €$ & $-55,96 \%$ \\
\hline
\end{tabular}

Quelle: eigene Darstellung 
Für die Aufteilung des Gesamtfördervolumens sind nach der Bund-LänderVereinbarung (BLV 2013) über das gemeinsame Programm der Qualitätsoffensive Lehrerbildung die Länderquoten nach dem Königssteiner Schlüssel sowie die Zahl der Lehramtsabsolventen zu beachten. Da die entsprechenden Zahlen der Lehramtsabsolventen nicht veröffentlicht sind, wird die Verteilung zunächst nur auf Basis des Königssteiner Schlüssel analysiert. Für die Ermittlung der Förderanteile der Länder gem. Königssteiner Schlüssel (vgl. Tab. 1) wurde das zur Förderung empfohlene Gesamtfördervolumen der dritten Förderphase in Höhe von rund 77 Millionen Euro zugrunde gelegt. Die ermittelten Länderanteile sind in der dritten Spalte der Tabelle 1 (Förderanteil gem. Königssteiner Schlüssel) ausgewiesen.

Insgesamt ergeben sich teilweise erhebliche Abweichungen vom Königssteiner Schlüssel, das verdeutlicht die relative Differenz der Fördersumme zum Länderanteil (letzte Spalte der Tab. 1). Die relative Abweichung liegt zwischen -75 und +96 Prozent. Für Brandenburg und Hamburg liegt die Differenz sogar bei -100 Prozent, weil keine Projektskizzen zur Förderung empfohlen wurden. Diese beiden Länderanteile mussten entsprechend auf die anderen Länder verteilt werden. Das erklärt jedoch nicht die teilweise erheblichen Differenzen der zugewiesenen Fördersummen zum Länderanteil. So wurde beispielsweise der Förderanteil von Berlin gem. Königssteiner Schlüssel um knapp 75\% unterschritten, während dagegen die Förderanteile von Bremen und Sachsen gem. Königssteiner Schlüssel um rund 76\% (Bremen) bzw. 96\% (Sachsen) überschritten wurden.

Erklärbar sind diese Abweichungen wahrscheinlich über die in der BLV (2013) festgesetzte Erweiterung der Bemessungsgrundlage des Königssteiner Schlüssels um die anteilsmäßige Zahl der Lehramtsabsolventen der Länder. Da diese Daten nicht vorliegen, lässt sich dies jedoch nicht weiter nachvollziehen.

\section{Die Phase der Antragsstellung und die Auswahlentscheidungen der dritten Förderphase der Qualitätsoffensive Lehrerbildung aus der Perspektive der Sektion Berufs- und Wirtschaftspädagogik}

Bereits während der Phase der Erarbeitung und Einreichung von Projektskizzen wurde der Vorstand der Sektion BWP in der DGfE mit Sorge darüber informiert, dass an einigen Universitäten und Pädagogischen Hochschulen die Förderrichtlinie zur dritten Förderphase der Qualitätsoffensive Lehrerbildung nur in Richtung Digitalisierung und Allgemeine Lehrerbildung aufgegriffen werden sollte und weitere Antragsmöglichkeiten für die berufliche Lehrerbildung nicht möglich gewesen seien. Um sich einen Überblick über die Situation 
der Antragsstellung an den unterschiedlichen Standorten zu verschaffen und die Auswahlentscheidung für den Schwerpunkt Lehrerbildung für berufliche Schulen zu dokumentieren, wurden noch im Zeitraum der Antragserstellung (im Dezember 2019) sowie zusätzlich nach Bekanntgabe der Auswahlentscheidung (ab Ende Mai 2019) kurz gefasste Online-Umfragen zu den Erfahrungen der Kolleg* der beruflichen Lehrerbildung an den Universitäten / Pädagogischen Hochschulen und Fachhochschulen ${ }^{5}$ durchgeführt. Zur Teilnahme an den Umfragen aufgefordert waren alle Kolleg*innen, die zu diesen Zeitpunkten an den universitären / hochschulischen Studienstandorten Verantwortung für die Studiengänge des Lehramts an berufsbildenden Schulen getragen haben. Ziel war es, zunächst die Auswahlentscheidung aus der Perspektive dieser Personen zu dokumentieren, um seitens des Vorstandes der Sektion BWP gegebenenfalls gegenüber dem BMBF und der GWK Stellung zum Verfahren und zum Ergebnis des Auswahlverfahrens beziehen zu können.

An der ersten Umfrage des Sektionsvorstandes beteiligten sich 14 Standortvertreter*innen. Fokus der Umfrage waren strategische Überlegungen und Entscheidungen der Universitäten und Pädagogischen Hochschulen zur Antragserarbeitung für die dritte Förderphase. Im Ergebnis zeigte sich, dass an einigen Standorten $(n=3)$ teilweise ohne jegliche Beteiligung der zuständigen Arbeitsbereiche / Institute beschlossen wurde, die Förderlinie zur dritten Förderphase der Qualitätsoffensive Lehrerbildung nur in Richtung Digitalisierung und Allgemeine Lehrerbildung aufzugreifen und darauf bezogen einen Einzelantrag und einen Verbundantrag auf den Weg zu bringen. Damit waren dort, aufgrund der Beschränkungen in der Ausschreibung, weitere Antragsmöglichkeiten für die berufliche Lehrerbildung nicht mehr möglich. Zwei Standortvertreter*innen berichteten weiterhin, dass es anfänglich solche Tendenzen zur Fokussierung der allgemeinen Lehrerbildung gegeben habe, nach ausreichender Intervention jedoch Einzel- oder Verbundanträge für die berufliche Lehrerbildung erarbeitet werden konnten.

Die zweite Umfrage wurde von insgesamt 26 Standorten beantwortet, die insgesamt 35 Anträge eingereicht hatten. Nach eigenen Angaben hatten drei Standorte (12\%) keinen Antrag, 13 Standorte (50\%) eine Antragsskizze und

5 Fachhochschulen waren selbst nicht antragsberechtigt, konnten aber als Partner in der Lehrerbildung in den Anträgen der Universitäten und Pädagogischen Hochschulen berücksichtigt werden. 
elf Standorte (38\%) Skizzen für einen Einzel- und einen Verbundantrag ${ }^{6}$ eingereicht. Die Anträge adressierten sowohl den Schwerpunkt berufliche Lehrerbildung als auch den Schwerpunkt Digitalisierung oder beide Schwerpunkte. Projektskizzen im Schwerpunkt berufliche Lehrerbildung wurden dabei überwiegend als Einzelanträge eingereicht und Projekte im Schwerpunkt Digitalisierung eher als Verbundanträge auf den Weg gebracht (vgl. Abb. 4). Dieser Befund deckt sich in Bezug auf die Einreichungen im Schwerpunkt berufliche Lehrerbildung mit den veröffentlichten Daten zur allgemeinen Antragssituation im Rahmen der dritten Förderrunde (vgl. Abschnitt 4).

Haben Standorte zwei Projektskizzen eingereicht, wurde in mehr als der Hälfte der Fälle ( $\mathrm{n}=6,55 \%)$ auf beide Schwerpunkte reagiert, wobei die berufliche Lehrerbildung eher in einem Einzelantrag und der Schwerpunkt Digitalisierung in einem Verbundantrag aufgegriffen wurde. Nur zwei Standorte gaben an, sowohl einen Einzel- als auch einen Verbundantrag im Schwerpunkt berufliche Lehrerbildung eingereicht zu haben. Dass der Schwerpunkt berufliche Lehrerbildung ,nur“ in einem Verbundantrag aufgegriffen wurde, gab nur ein Standort an.

\section{Abb. 4: Eingereichte Anträge nach Antragsform und Schwerpunkt}

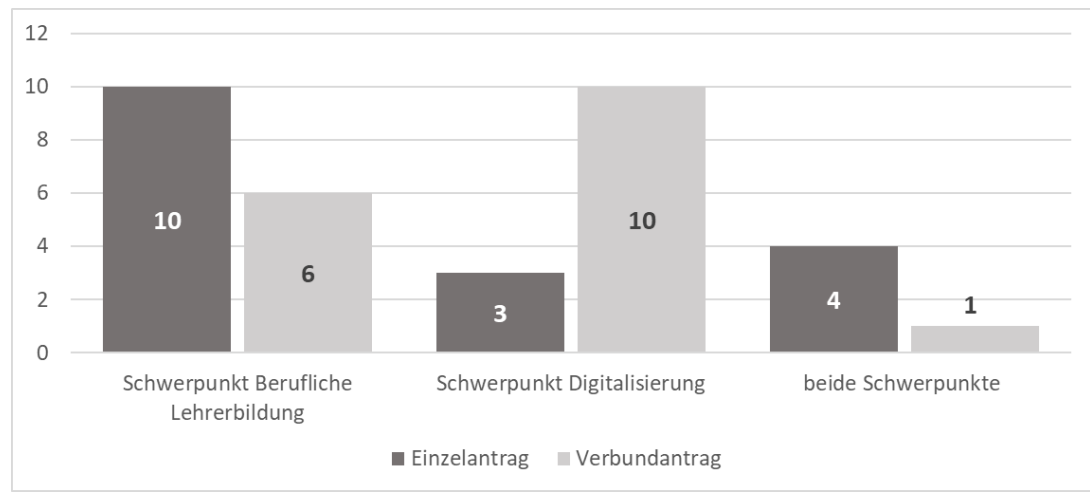

Quelle: eigene Darstellung

6 Für die Anzahl der eingereichten Verbundanträge ergeben sich aus dieser Erhebung sowohl für den Schwerpunkt berufliche Lehrerbildung als auch für den Schwerpunkt Digitalisierung Differenzen zu den veröffentlichten Angaben zur Antragssituation nach Schwerpunkten (vgl. Abschnitt 4). Dort wurden für den Schwerpunkt berufliche Lehrerbildung beispielsweise drei eingereichte Verbundprojektskizzen ausgewiesen, in der Sektionsumfrage gaben sechs Standorte an, Verbundanträge im Schwerpunkt berufliche Lehrerbildung eingereicht zu haben. Es scheinen also jeweils mehrere Verbundpartner an der Befragung des Sektionsvorstandes teilgenommen zu haben; dies kann nicht abschließend geprüft werden, da nur die Projektpartner der zur Förderung empfohlenen Verbundprojekte bekannt sind. 
Von den insgesamt 35 Projektskizzen wurden 24 Skizzen als förderfähig bewertet (69\%). Nicht förderfähig waren überwiegend Skizzen aus dem Schwerpunkt berufliche Lehrerbildung $(n=6,37,5 \%$ der im Schwerpunkt eingereichten Projektskizzen der Befragten). Unterschiede zu den Befunden zur allgemeinen Antragssituation (Abschnitt 4) ergeben sich aufgrund der unterschiedlichen Grundgesamtheit.

Auch in der zweiten Umfrage des Sektionsvorstands, die von insgesamt 23 Personen beantwortet wurde, wurde die Frage aufgegriffen, ob aus der Perspektive der Standortvertreter*innen die Möglichkeit bestanden habe, einen Antrag für den Schwerpunkt berufliche Lehrerbildung einzureichen. Für drei Standorte, darunter nur ein Standort, der dies bereits in der ersten Umfrage zurückmeldete, wurde angegeben, dass institutionelle Präferenzen für Digitalisierung und allgemeinbildendes Lehramt eine Antragsstellung unter dem Schwerpunkt der beruflichen Lehrerbildung verhindert hätten. Für die überwiegende Mehrheit der Teilnehmenden $(n=23)$ hat es entweder keine Interessenkonflikte gegeben oder diese führten nicht dazu, dass ein Antrag für den Schwerpunkt der beruflichen Lehrerbildung verhindert wurde.

\section{Fazit}

Die berufliche Lehrerbildung erhält mit der dritten Förderrunde der Qualitätsoffensive Lehrerbildung hohe Aufmerksamkeit. Insgesamt 17 Projekte der letzten Förderrunde der Qualitätsoffensive Lehrerbildung werden sich intensiv mit der Ausbildung beruflicher Lehrkräfte beschäftigen, das sind immerhin knapp 39 Prozent der förderfähigen Projekte. Es kann angenommen werden, dass auch eine Reihe der Projekte aus dem Schwerpunkt Digitalisierung das berufliche Lehramt adressieren, sodass der Anteil der geförderten Projekte, die über die dritte Förderphase der Qualitätsoffensive Lehrerbildung einen Innovationsschub für die berufliche Lehrerbildung leisten, noch höher liegen wird, allerdings mit Dominanz im Bereich der Digitalisierungsthematik und möglicherweise zu Lasten anderer für die berufliche Lehrerbildung qualitätsrelevanten Thematiken. Investiert werden mindestens rund 21,6 Millionen Euro in die Weiterentwicklung der beruflichen Lehrerbildung, davon rund 19,9 Millionen Euro für Projekte im Schwerpunkt berufliche Lehrerbildung und rund 1,7 Millionen Euro für Projekte, die sich beiden Schwerpunkten (Digitalisierung und berufliche Lehrerbildung) zuordnen lassen. Mit letzterem ist möglicherweise verbunden, dass spezifische Thematiken der Digitalisierung, insbesondere der Digitalisierung der Arbeits- und Berufswelt, im Bereich der beruflichen Lehrerbildung eher randständig thematisiert werden. 
Mit über 72 Prozent positiv beschiedener Förderprojekte im Schwerpunkt berufliche Lehrerbildung und 50 Prozent positiver Entscheidungen für Anträge, die sich beiden Schwerpunkten zuordnen lassen, ist die Quote der zur Förderung empfohlenen Anträge (gemessen an allen im Schwerpunkt eingereichten Anträgen) höher als für den Schwerpunkt Digitalisierung (65\%), wo jedoch mit 66 Projektskizzen deutlich mehr Einreichungen vorlagen. Disparitäten ergeben sich durchaus in Bezug auf das Fördervolumen, das für den Schwerpunkt berufliche Lehrerbildung auch in der dritten Förderrunde geringer ausfällt als das Fördervolumen, das im Schwerpunkt Digitalisierung investiert wird. Wobei für letzteren auf Basis der vorliegenden Daten unklar bleibt, in welchem Umfang auch hier die berufliche Lehrerbildung adressiert wird. Das durchschnittliche Fördervolumen der einzelnen (Einzel- oder Verbund-)Projekte fällt für den Schwerpunkt berufliches Lehramt im Vergleich zum Schwerpunkt Digitalisierung geringer aus. Offen bleibt in diesem Zusammenhang, ob die Verantwortlichen an den Standorten der beruflichen Lehrerbildung hier weniger mutig notwendige Mittel beantragt haben, tatsächliche Fördermittelbedarfe realistischer eingeschätzt haben oder aber durch universitäre bzw. hochschulische oder gar ministerielle Präferenzen begrenzt wurden.

Insgesamt ist jedoch festzustellen, dass es mit der Fokussierung der beruflichen Lehrerbildung als ein Schwerpunkt der Förderrichtlinie gelungen ist, deutlich mehr Projekte zu initiieren, die sich ausschließlich auf das berufliche Lehramt beziehen. Allein mit den Projekten im Schwerpunkt berufliche Lehrerbildung wird eine um zehn Prozentpunkte höhere Förderquote (26\%) realisiert als in der zweiten Förderrunde für das gesamte berufliche Lehramt (anteilsmäßig einbezogen wurden in die Ermittlung der Förderquote hier alle Projekte, in denen das berufliche Lehramt adressiert wurde/wird; die geschätzte Förderquote lag bei 16\%, vgl. Abschnitt 3).

Da bisher keine Informationen zu den Ansätzen der unterschiedlichen Projekte veröffentlicht sind, lässt sich noch nicht abschätzen, welche Handlungsfelder für die berufliche Lehrerbildung aufgegriffen werden. Begrüßenswert ist, dass die Sichtbarkeit und Wertschätzung der beruflichen Lehrerbildung im Programm der Qualitätsoffensive Lehrerbildung gestiegen sind. Auch in der Programmbegleitung sollen die besonderen Herausforderungen und Problemlagen der beruflichen Lehrerbildung stärker als bisher aufgegriffen werden. So ist für September 2020 ein erster Workshop zu aktuellen Ansätzen und Entwicklungen in der beruflichen Lehrerbildung vorgesehen und angekündigt, dass weitere auf die berufliche Lehrerbildung fokussierte Workshops folgen werden. 


\section{Literatur}

BLV (2013). Bund-Länder-Vereinbarung über ein gemeinsames Programm „Qualitätsoffensive Lehrerbildung " gemäß Artikel 91 b des Grundgesetzes vom 12.04.2013.

Bundesministerium für Bildung und Forschung (BMBF) (o. J. a). Qualitätsoffensive Lehrerbildung - Ergebnis der Sitzung des Auswahlgremiums am 22./23.02.2018 in Berlin (2. Förderphase, 1. Runde). Online: https:/www.qualitaetsoffensivelehrerbildung.de/files/QLB-Auswahlergebnis_2018-02-23_.pdf (06.12.2019).

Bundesministerium für Bildung und Forschung (BMBF) (o. J. b). Qualitätsoffensive Lehrerbildung - Ergebnis der Sitzung des Auswahlgremiums am 01./02.10.2018 in Potsdam (2. Förderphase, 2. Runde). Online: https:/www.qualitaetsoffensivelehrerbildung.de/files/QLB-Auswahlergebnis_2018-10-02.pdf (06.12.2019).

Bundesministerium für Bildung und Forschung (BMBF) (o. J. c). Verbundvorhaben Perspektive Lehramt als neue Chance. Online: https:/www.qualitaetsoffensivelehrerbildung.de/de/projekte.php?D=9\#dtl (06.12.2019).

Bundesministerium für Bildung und Forschung (BMBF) (o. J. d). TUB Teaching $2.0-$ Innovativer Einstieg, Professions- und Forschungsorientierung im berufsbezogenen Lehramtsstudium. Online: https:/www.qualitaetsoffensive-lehrerbildung.de/de/projekte.php?C $=2 \& \mathrm{D}=57$ (06.12.2019).

Bundesministerium für Bildung und Forschung (BMBF) (2014). Richtlinie zur Förderung der „Qualitätsoffensive Lehrerbildung“ vom 10. Juli 2014. https://www.bmbf.de/foerderungen/bekanntmachung-951.html (13.12.2019).

Brümmer, F., Durdel, A., Fischer-Münnich, C., Fittkau, J. \& Weiger, W. (2018). Qualitätsoffensive Lehrerbildung. Zwischenbericht der Evaluation. Hamburg: Ramboll Management Consulting.

Drucksache 19/11886 des Bundestags vom 24.07.2019. Antwort der Bundesregierung auf die Kleine Anfrage der Abgeordneten Dr. Jens Brandenburg (Rhein-Neckar), Katja Suding, Grigorios Aggelidis, weiterer Abgeordneter und der Fraktion der FDP (Drucksache 19/11437): Qualitätsoffensive Lehrerbildung in der beruflichen Bildung. Online: http://dipbt.bundestag.de/dip21/btd/19/118/1911886.pdf (12.12.2019).

Frommberger, D. \& Lange, S. (2018). Zur Ausbildung von Lehrkräften an berufsbildenden Schulen - Befunde und Entwicklungsperspektiven. Working Paper Forschungsförderung (Hans-Böckler-Stiftung), 60.

Gillen, J., Wasserschleger, A., Wehking, K. \& Beinke, K. (2018). Zwischen Ingenieurstudium und Lehramtsoption - Wann und warum entscheiden sich Studierende für den „PLan C“? Journal of Technical Education, 6(2), 25-38.

Gemeinsame Wissenschaftskonferenz (GWK) (2019). Qualitätsoffensive Lehrerbildung: neue Förderrunde startet. Pressemitteilung 07/2019. https://www.gwkbonn.de/fileadmin/Redaktion/Dokumente/Pressemitteilungen/pm2019-07.pdf (13.12.2019).

Klemm, K. (2018). Dringend gesucht: Berufsschullehrer. Die Entwicklung des Einstellungsbedarfs in den beruflichen Schulen in Deutschland zwischen 2016 und 2035. Gütersloh: Bertelsmann-Stiftung. 
Lange, S. \& Frommberger, D. (2020). Lehrkräfteentwicklung an berufsbildenden Schulen in Deutschland. Befunde zu Studierendenzahlen, Einstellungsbedarfen und Seiteneinstiegen. In R. Koerber \& B. Groot-Wilken (Hrsg.), Nachhaltige Professionalisierung für Lehrerinnen und Lehrer: Ideen, Entwicklungen, Konzepte (S. 121-146). Bielefeld: wbv.

Lipsmeier, A. (2014). Bachelorlehrer - eine Radikalkur zur Behebung des Gewerbelehrermangels als letzte Therapie nach ernüchternder Diagnose. Die berufsbildende Schule, 66(7/8), 252-258.

Terhart, E. (2000). Perspektiven der Lehrerbildung in Deutschland. Abschlussbericht der von der Kultusministerkonferenz eingesetzten Kommission. Weinheim u. a.: Beltz. 



\section{Herausgeberschaft}

Prof. Dr. Eveline Wittmann

Technische Universität München

Lehrstuhl für Berufspädagogik

eveline.wittmann@tum.de

Prof. Dr. Dietmar Frommberger

Universität Osnabrück

Professur für Berufs- und Wirtschaftspädagogik

dietmar.frommberger@uni-osnabrueck.de

Prof. Dr. Ulrike Weyland

Westfälische Wilhelms-Universität Münster

Professur für Erziehungswissenschaft

Schwerpunkt Berufspädagogik

ulrike.weyland@uni-muenster.de

\section{Autorinnen und Autoren}

Prof. Dr. Carmela Aprea

Universität Mannheim

Lehrstuhl für Wirtschaftspädagogik

Design und Evaluation instruktionaler Systeme

aprea@bwl.uni-mannheim.de

Prof. Dr. Rer. Soc. Karin Reiber

Hochschule Esslingen

Fakultät für soziale Arbeit, Gesundheit und Pflege

karin.reiber@hs-esslingen.de

PD Dr. habil. Florian Berding

Carl von Ossietzky Universität Oldenburg

Fakultät II Department für Wirtschafts- und Rechtswissenschaften

Fachgebiet Berufs- und Wirtschaftspädagogik

florian.berding@uni-oldenburg.de 
Josephine Berger, M.Sc. psych

Technische Universität Darmstadt

Institut für Allgemeine Pädagogik und Berufspädagogik

berger@bp.tu-darmstadt.de

Dr. Dana Bergmann

Otto-von-Guericke-Universität Magdeburg

Institut Bildung - Beruf - Medien

Professur für Wirtschaftsdidaktik und Didaktik der ökonomischen Bildung dana.bergmann@ovgu.de

Prof. Dr. Thomas Bienengräber

Universität Duisburg-Essen

Lehrstuhl für Wirtschaftspädagogik und Wirtschaftsdidaktik

thomas.bienengraeber@uni-due.de

Leonie Bogaczyk, M.A.

Universität Duisburg-Essen, Campus Essen

Lehrstuhl für Wirtschaftswissenschaften und Didaktik der Wirtschaftslehre leonie.bogaczyk@uni-due.de

Prof. Dr. Andrea Burda-Zoyke

Christian-Albrechts-Universität zu Kiel

Professur für Berufs- und Wirtschaftspädagogik

burda-zoyke@paedagogik.uni-kiel.de

Dr. phil. des. Marc Casper

Universität Hamburg

Fakultät für Erziehungswissenschaft

Berufliche Bildung und Lebenslanges Lernen (EW 3)

marc.casper@uni-hamburg.de

Immo Degner, M.A.

Christian-Albrechts-Universität zu Kiel

Abt. Berufs- und Wirtschaftspädagogik am Institut für Pädagogik (IfP)

degner@paedagogik.uni-kiel.de

Prof. Dr. Andreas Fischer

Leuphana Universität Lüneburg

Arbeitseinheit Wirtschaftspädagogik 
Prof. Dr. Manuel Förster

Technische Universität München

Professur Wirtschaftspädagogik

manuel.foerster@tum.de

Prof. Dr. Bärbel Fürstenau

TU Dresden

Professur für Wirtschaftspädagogik

baerbel.fuerstenau@tu-dresden.de

Harald Hantke, M.Ed.

Leuphana Universität Lüneburg

Arbeitseinheit Wirtschaftspädagogik

Professur für Berufs- \& Wirtschaftspädagogik

sowie Didaktik der Wirtschaftslehre

harald.hantke@leuphana.de

Vertr.-Prof. Dr. Mandy Hommel

Otto-Friedrich-Universität Bamberg

Vertr. Professur für Wirtschaftspädagogik

mandy.hommel@uni-bamberg.de

Dr. rer. pol. Heike Jahncke

Carl von Ossietzky Universität Oldenburg

Fakultät II Department für Wirtschafts- und Rechtswissenschaften

Fachgebiet Berufs- und Wirtschaftspädagogik

heike.jahncke@uni-oldenburg.de

Karina Kiepe, M.Ed.

Helmut-Schmidt-Universität

Fakultät für Geistes- und Sozialwissenschaften

Berufs- und Arbeitspädagogik

kiepek@hsu-hh.de

StR Susanne Korth

RWTH Aachen University

Lehr- und Forschungsgebiet Fachdidaktik Bautechnik

korth@fdb.rwth-aachen.de

Andreas Kraitzek, M.Sc.

Technische Universität München

Professur Wirtschaftspädagogik

andreas.kraitzek@tum.de 
Dr. Silke Lange

Universität Osnabrück

Fachbereich Erziehungs- und Kulturwissenschaften

Abteilung Berufs- und Wirtschaftspädagogik

silke.lange@uni-osnabrueck.de

Jutta Mohr, M.A.

Hochschule Esslingen

Fakultät Soziale Arbeit, Gesundheit und Pflege

jutta.mohr@hs-esslingen.de

Svenja Noichl, M.Sc.

RWTH Aachen University

Lehr- und Forschungsgebiet Informatik 9 - Learning Technologies

noichl@informatik.rwth-aachen.de

Prof. Dr. Jane Porath

Hochschule der Bundesagentur für Arbeit

Pädagogik

Schwerpunkt: Berufs- und Wirtschaftspädagogik

Jane.Porath@arbeitsagentur.de

Jan Pranger, M.Ed.

Leuphana Universität Lüneburg

Arbeitseinheit Wirtschaftspädagogik

Professur für Berufs- \& Wirtschaftspädagogik

sowie Didaktik der Wirtschaftslehre

jan.pranger@leuphana.de

Prof. Dr. Karin Reiber

Hochschule Esslingen

Professorin für Erziehungswissenschaft/Didaktik

Karin.Reiber@hs-esslingen.de

Prof. Dr. Thomas Retzmann

Universität Duisburg-Essen, Campus Essen

Lehrstuhl für Wirtschaftswissenschaften und Didaktik der Wirtschaftslehre thomas.retzmann@uni-due.de 
Univ.-Prof. Dr. Volker Rexing

RWTH Aachen University

Fakultät für Bauingenieurwesen

Lehr- und Forschungsgebiet Fachdidaktik Bautechnik

rexing@fdb.rwth-aachen.de

Isabelle Riedlinger, Magister

Hochschule Esslingen

Fakultät Soziale Arbeit, Gesundheit und Pflege

Isabelle.Riedlinger@hs-esslingen.de

Dr. Michelle Rudeloff

Eberhard-Karls-Universität Tübingen

Wirtschafts- und Sozialwissenschaftliche Fakultät

Ökonomische Bildung und Wirtschaftsdidaktik

michelle.rudeloff@uni-tuebingen.de

Andrea Sailer

Österreichische Autorin

Kontakt über Prof. Dr. Michaela Stock

Karl-Franzens-Universität Graz

Institut für Wirtschaftspädagogik

michaela.stock@uni-graz.at

Marie Schröder

Universität Duisburg-Essen

Lehrstuhl für Wirtschaftspädagogik und Wirtschaftsdidaktik

marie.schroeder@uni-due.de

Sören Schütt-Sayed

Universität Hamburg

Fakultät für Erziehungswissenschaft

Berufspädagogik

Berufliche Bildung und Lebenslanges Lernen (EW 3)

soeren.schuett@uni-hamburg.de

Christin Siegfried

Goethe-Universität Frankfurt

Wirtschaftswissenschaften, Fachbereich 02

Vertretung der Juniorprofessur für Wirtschaftspädagogik

siegfried@em.uni-frankfurt.de 
Dr. Christian Steib

Carl von Ossietzky Universität Oldenburg

Fakultät II Department für Wirtschafts- und Rechtswissenschaften

Fachgebiet Berufs- und Wirtschaftspädagogik

christian.steib@uol.de

Prof. Dr. Eveline Wuttke

Goethe-Universität Frankfurt

Wirtschaftswissenschaften, Fachbereich 02

Wirtschaftspädagogik

wuttke@em.uni-frankfurt.de

Prof. Dr. Birgit Ziegler

Technische Universität Darmstadt

Institut für Allgemeine Pädagogik und Berufspädagogik

ziegler@bp.tu-darmstadt.de 
Inklusionsforschung im

Spannungsfeld von

Erziehungswissenschaft

und Bildungspolitik

Jurgen Budde, Andrea Dlugosch, Petra Herzmann.

Lisa Rosen, Argyro Panagioto, Tanja Sturm.

Schriftenreih

der AG Inklusionsforschung der Deutschen Gesellschaft for Erziehungswissenschaft (DGFE)

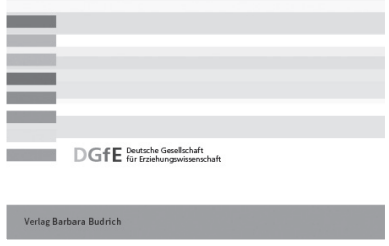

Jürgen Budde u.a.

Inklusionsforschung im Spannungsfeld von Erziehungswissenschaft und Bildungspolitik

Schriftenreihe der AG Inklusion der Deutschen Gesellschaft für

Erziehungswissenschaft (DGfE)

2019. 204 Seiten $\cdot$ Kart. $\cdot 28,00 €(D) \cdot 28,80 €(A)$

ISBN 978-3-8474-2201-3 • eISBN 978-3-8474-1227-4

Der Band reflektiert Inklusion als Gegenstand bildungstheoretischer und sozialwissenschaftlicher Forschung. Darüber hinaus konturiert er die Inklusionsforschung als eigenes erziehungswissenschaftliches Feld. Gegenwärtige Inklusionsforschung realisiert sich damit in Spannungsfeldern, die in unterschiedlichen Bereichen der Erziehungswissenschaft in verschiedener Weise verhandelt werden. 


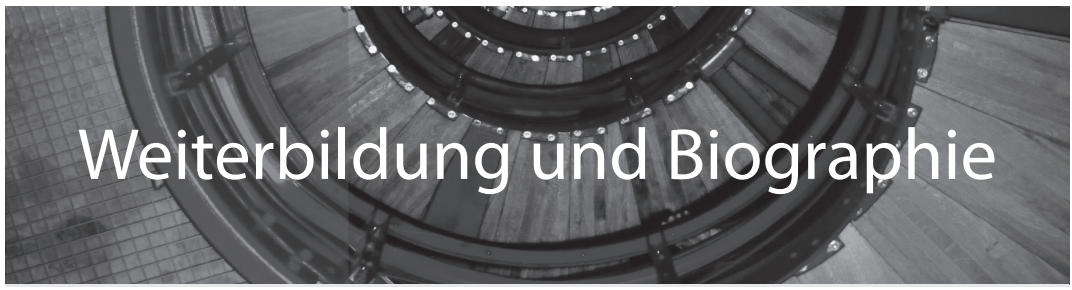

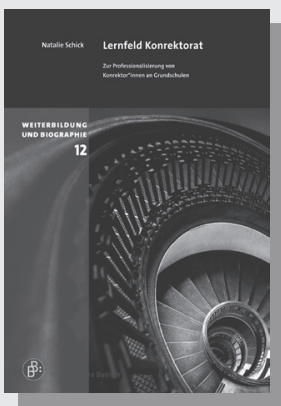

Band 12

$2020 \cdot 444$ Seiten $\bullet$ Kart. $\bullet$

$52,00 €(D) \cdot 53,50 €(A)$

ISBN 978-3-8474-2361-4

eISBN 978-3-8474-1531-2

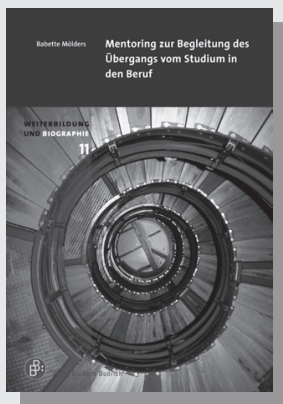

Band 11

2018 380 Seiten $\bullet$ Kart. $\quad 2015 \cdot 356$ Seiten $\bullet$ Kart. • $43,00 €(D) \cdot 44,30 €(A) \quad 44,00 €(D) \cdot 45,30 €(A)$ ISBN 978-3-8474-2125-2 ISBN 978-3-8474-0764-5 eISBN 978-3-8474-1113-0 eISBN 978-3-8474-0882-6

Die Reihe "Weiterbildung und Biographie" diskutiert auf Basis einer erziehungswissenschaftlich orientierten Biographieforschung aktuelle Themen und Probleme der Weiterbildung. Biographien liefern Deutungen sozialer Wirklichkeit und beleuchten das aktuelle Verständnis der Lebenswelt, das für Bildungsarbeit im Erwachsenenalter relevant ist. Biographieorientierte empirische Forschungen gewähren dabei Einblicke in unterschiedliche Felder der sozialen Wirklichkeit, die in der Weiterbildung zum Thema werden.

\section{B: \\ www.shop.budrich.de}




\section{E. Wittmann, D. Frommberger, U. Weyland (Hrsg.)}

\section{Jahrbuch der berufs- und \\ wirtschaftspädagogischen Forschung 2020}

Wie entwickelt sich die Forschung in der Berufs- und Wirtschaftspädagogik? Das Jahrbuch gibt einen Überblick über den aktuellen Stand und bildet dadurch das breite thematische und methodologische Spektrum der Forschung und Theoriebildung im Fachbereich ab. Der diesjährige Band versammelt Beiträge, die bei der Tagung der Sektion Berufs- und Wirtschaftspädagogik der DGfE erstmalig einer breiteren Fachöffentlichkeit präsentiert wurden. Die Beiträge wurden einem Reviewverfahren unterzogen.

Die Herausgeber*innen:

Prof. Dr. Eveline Wittmann, Lehrstuhl für Berufspädagogik, Technische Universität München

Prof. Dr. Dietmar Frommberger, Professur für Berufs- und Wirtschaftspädagogik, Universität Osnabrück

Prof. Dr. Ulrike Weyland, Professur für Erziehungswissenschaft mit dem Schwerpunkt Berufspädagogik, Westfälische WilhelmsUniversität Münster 